\title{
Copyright
}

by

Bonnie Elise Canion

2015 
The Dissertation Committee for Bonnie Elise Canion certifies that this is the approved version of the following dissertation:

\section{Incorporation of Photon Analysis into an Active Interrogation System for Shielded Uranium Characterization}

Committee:

Sheldon Landsberger, Supervisor

Seth McConchie, Co-Supervisor

Erich Schneider

Steven Biegalski

Jason Hayward 


\title{
Incorporation of Photon Analysis into an Active Interrogation System for Shielded Uranium Characterization
}

\author{
by \\ Bonnie Elise Canion, B.S.Physics; M.S.E.
}

\author{
Dissertation \\ Presented to the Faculty of the Graduate School of \\ The University of Texas at Austin \\ in Partial Fulfillment \\ of the Requirements \\ for the Degree of
}

Doctor of Philosophy

The University of Texas at Austin

May 2016 


\section{Dedication}

To my parents, who have always given me endless love and support. I know that the foundations of my success are rooted in Times Tables flashcards, piano lessons, girl scout troop camping trips, the adventures of Bilbo Baggins and Hank the Cowdog, and all my other lovely childhood memories.

To my fiance, Steve. You took on the burden of this work with me and selflessly supported me through the ups and downs. You celebrated my successes and encouraged me when I felt defeated. You took on all other tasks to allow me to focus on my work. I have such admiration for your patience and compassion. Let's get married! 


\section{Acknowledgements}

Thank you to Sheldon Landsberger, who is one of the reasons I find myself in this field today. Thank you for encouraging me to apply for the Nuclear Engineering Program and guiding me ever since. I did not know that I was capable of accomplishing all that I have, and am grateful for you seeing potential in me and setting me onto this course.

Thank you to Seth McConchie, my mentor for this dissertation work. I am fortunate to have had the opportunity to learn from someone whom I admire and respect. Thank you for for teaching me and for challenging me. I am certainly finishing this dissertation as a more competent and confident scientist because of your guidance.

Thank you also to the Nuclear Engineering Program staff and students, especially those of the Nuclear Engineering Teaching Laboratory. Your support and friendship made my time in grad school productive and enjoyable. Thank you to the other graduate students, especially Franziska Klingberg, who were a great source for either collaborating with or eating queso with, depending on the type of day it had been.

I am also grateful to the Nuclear Material Detection and Characterization Group at Oak Ridge National Laboratory for giving me their time and expertise, especially John Mihalczo, Jim Mullens, and James Henkel. Thank you to the graduate students at ORNL for being there to lend a hand in the lab, discuss concepts, or make me laugh, especially Tracey Wellington, Matthew Tweardy, and Blake Palles. A special thank you to Tracey for all of her feedback on this dissertation and presentation material, and for her friendship and support. Thank you also to Deb Austin, Marc Hampton, and Gomez Wright for their support and friendship while I was at ORNL, 
which included everything from ensuring I had everything I needed for research to feeding me or making me laugh.

Thank you also to everyone in the Defense Nuclear Nonproliferation Research and Development (NA-22) office for their support and understanding while I completed this work, especially my manager, Marco DiCapua. Your words of encouragement and congratulations have meant a lot to me.

Lastly, thank you to the Nuclear Forensics Graduate Fellowship for funding this work*.

*This material is based upon work supported by the U.S. Department of Homeland Security under Grant Award Number, 2012-DN-130-NF0001.

The views and conclusions contained in this document are those of the authors and should not be interpreted as representing the official policies, either expressed or implied, of the U.S. Department of Homeland Security. 


\title{
Incorporation of Photon Analysis into an Active Interrogation System for Shielded Uranium Characterization
}

\author{
Bonnie Elise Canion, Ph.D. \\ The University of Texas at Austin, 2015
}

Supervisor: Sheldon Landsberger

Co-Supervisor: Seth McConchie

In this project I explored the ability of induced photon and neutron signatures from an associated particle imaging deuterium tritium (API-DT) neutron generator detection system to assess the enrichment of shielded uranium. Fast electronics and the associated particle technique, which provide the timing and directional information of the source $14 \mathrm{MeV}$ neutron, allow for a correlated time window of tens of nanoseconds, thus enabling extraction of clear prompt fission signatures from the inspected object.

This project contains three distinct investigations that involve relating API-DT neutron generator induced signatures to uranium enrichment.

1. Explore the correlated neutron and photon detection rates, separated by timeof-flight, and investigate the use of a 3-D radiation transport Monte Carlo code to predict enrichment, based on these signatures, 
2. Examine the same correlated detection rates, but use a simple point kinetics technique to relate signatures to enrichment, and

3. Exploit the prompt photon energy spectrum to determine if fission neutrons activate shielding material to provide a signature that can be related to uranium enrichment.

Throughout these three studies, I investigated the time, number, and energy distribution of these prompt photon signatures, which enabled me to draw conclusions about the strengths and weaknesses of these signatures for shielded uranium characterization. 


\section{Table of Contents}

List of Figures $\ldots \ldots \ldots \ldots \ldots \ldots \ldots \ldots \ldots \ldots \ldots$

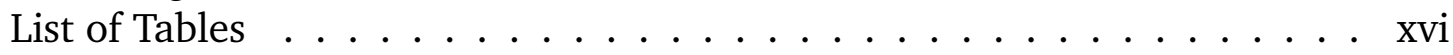

1 Introduction 1

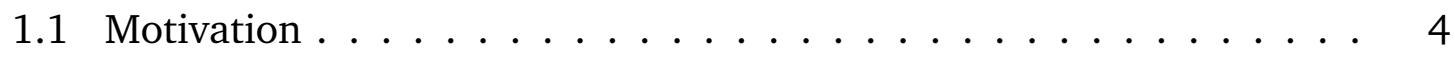

1.2 Background ................... 6

2 Time-of-Flight Signatures $\quad 16$

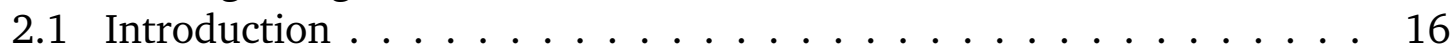

2.2 Previous Work . . . . . . . . . . . . . . . . . 18

2.3 Experimental Setup . . . . . . . . . . . . . . . . . . . . . 19

2.4 Analysis of Experimental Data . . . . . . . . . . . . . . 22

2.5 MCNP Simulations of API Detection System $\ldots \ldots \ldots \ldots$

2.6 Discussion . . . . . . . . . . . . . . . . . . . 46

3 Point Kinetics Analysis $\quad 48$

3.1 Introduction . . . . . . . . . . . . . . . 48

3.2 The Böhnel Equation . . . . . . . . . . . . . 53

3.3 Fission Chain Initiation . . . . . . . . . . . . . . 57

3.4 Photon Distribution . . . . . . . . . . . . . . . . . 62

3.5 Composite Equation . . . . . . . . . . . . . . . . 65

3.6 Adding Non-Fission Interactions . . . . . . . . . . . . . . 66

3.7 Adding Shielding . . . . . . . . . . . . . . . . . . 81

3.8 Modeling Detection Efficiency $\ldots \ldots \ldots \ldots \ldots$. . . . . . . . . . . . .

3.9 Determining Enrichment . . . . . . . . . . . . . . . . . 92

3.10 Effects of Input Values Uncertainty $\ldots \ldots \ldots \ldots$

3.11 Comparison to Experimental Data . . . . . . . . . . . . . . . 101

3.12 Discussion . . . . . . . . . . . . . . . . . . 102

4 Source Correlated Gamma-ray

$\begin{array}{ll}\text { Spectroscopy } & 106\end{array}$

4.1 Theory . . . . . . . . . . . . . . . . . . . . 107

4.2 Relevant Literature . . . . . . . . . . . . . . . . . . . . . . . 109

4.3 Identification and Localization of Material . . . . . . . . . 116 
4.4 Investigating Signatures from Shielded Uranium . . . . . . . . . . 121

4.5 Investigation of Signature Induced from Fission . . . . . . . . . . 125

4.6 Sodium Iodide Detector Measurements . . . . . . . . . . . . . 128

4.7 Discussion . . . . . . . . . . . . . . . . . . . . . . . 139

5 Conclusion 141

A Detector Position Sensitivity Characterization 145

A.1 Overview of Study . . . . . . . . . . . . . . . . 145

A.2 $14 \mathrm{MeV}$ Detector Characterization . . . . . . . . . . . . 147

A. $3{ }^{137}$ Cs Detector Characterization . . . . . . . . . . . . . . 156

A.4 Modifications to Post Processor . . . . . . . . . . . . 163

B Extended Experimental Setup 165

B.1 Calibrating the Fission Detectors . . . . . . . . . . . . 165

B.2 Setting the Alpha Detector Threshold . . . . . . . . . . . . . . . 169

B.3 Accurately Modeling NMIS . . . . . . . . . . . . . . . . 171

$\begin{array}{ll}C \text { Correction For Source Location } & 173\end{array}$

D High Purity Germanium Detector Damage 175

$\begin{array}{lll}\text { E PMT Gain Shift } & 177\end{array}$

$\begin{array}{ll}\text { Bibliography } & 180\end{array}$

$\begin{array}{ll}\text { Vita } & 192\end{array}$ 


\section{List of Figures}

1.1 Nuclear Material Identification System (1) . . . . . . . . . . . . 12

1.2 Source Correlated time-of-flight (2) . . . . . . . . . . . . . . . 14

1.3 Cross Sectional Image Created by NMIS (2) $\ldots \ldots \ldots \ldots$

2.1 Experimental setup used for measurement series, shown with the tungsten casting. . . . . . . . . . . . . . . . .

2.2 This series of plots shows how the signatures are deconvolved and what area of the spectrum is integrated to determine each signature rate. In each plot, the blue line is the data, source normalized and background subtracted; the red line is the sum of the fitted peaks to be subtracted out of the spectrum; and the black dashed line is the spectrum with the interfering peaks subtracted out. . . . . . . . . 23

2.3 Bicorrelation plot, with source-photon-photon, source-neutron-neutron, and source-photon-neutron signatures labeled. . . . . . . . . . . . 24

2.4 A comparison of DU and tungsten for all signatures. . . . . . . . 26

2.5 A study of DU signatures with two levels of aluminum shielding. . . 29

2.6 A study of DU signatures with two levels of lead shielding. . . . . . 30

2.7 A study of DU signatures with two levels of polyethylene shielding. . 31

2.8 The first interaction of the source particles were plotted for the input deck of the alpha pixel 8 field of view. . . . . . . . . . . . . 33

2.9 A comparison between data and simulation for all signatures. . . . . 34

2.10 All signatures with variable enrichments of bare uranium. . . . . . 39

2.11 All signatures with variable enrichments of uranium shielded by 2" of aluminum. . . . . . . . . . . . . . . . 40

2.12 All signatures with variable enrichments of uranium shielded by 2" of lead. . . . . . . . . . . . . . . . . .

2.13 All signatures with variable enrichments of uranium shielded by 2" of polyethylene. . . . . . . . . . . . . .

2.14 Example to illustrate the enrichment determinations in Tables 2.4 $2.7 \ldots \ldots \ldots \ldots \ldots \ldots \ldots \ldots \ldots$

3.1 Comparison of MCNPx PoliMi output to point kinetics model (Equation 3.19) of neutrons escaping from ${ }^{235} U$ metal spheres of 1,4 and $6 \mathrm{~cm}$ in radius respectively. . . . . . . . . . . . . . . 
3.2 Comparison of MCNPx PoliMi fission photons produced to point kinetics model (Equation 3.25) of photons produced within ${ }^{235} U$ metal spheres of 1,4 and $6 \mathrm{~cm}$ in radius respectively. . . . . . . . . .

3.3 Comparison of MCNPx PoliMi output to point kinetics model (Equation 3.31) of neutrons escaping from ${ }^{235} U$ metal spheres of 1,4 and $6 \mathrm{~cm}$ in radius respectively. . . . . . . . . . . .

3.4 Example to illustrate the enrichment determinations in Tables 3.1, with $(\mathrm{n}, 2 \mathrm{n})$ interactions included. . . . . . . . . . . . . 70

3.5 Comparison of MCNPx PoliMi output to point kinetics model (Equation 3.25 with absorption incorporated) of photons escaping from ${ }^{235} \mathrm{U}$ metal spheres of 1,4 and $6 \mathrm{~cm}$ in radius respectively. The consistent under-prediction of the point kinetics distributions is expected because inelastic scattering gamma-rays have not yet been modeled.

3.6 Comparison of MCNPx PoliMi output to point kinetics model (Equation 3.36) of photons escaping from ${ }^{235} U$ metal spheres of 1,4 and 6 $\mathrm{cm}$ in radius respectively. . . . . . . . . . . . . . .

3.7 Comparison of MCNPx PoliMi output to point kinetics model (Equation 3.36) of photons escaping from a ${ }^{235} \mathrm{U}$ metal sphere $4 \mathrm{~cm}$ radius with $5 \mathrm{~cm}$ of polyethylene shielding. . . . . . . . . .

3.8 Comparison of MCNPx PoliMi output to point kinetics model (Equation 3.36) of neutrons escaping from a ${ }^{235} \mathrm{U}$ metal sphere $4 \mathrm{~cm}$ radius with $5 \mathrm{~cm}$ of polyethylene shielding. The goal of including this plot is to demonstrate the lack of fit. . . . . . . . . . . . . .

3.9 Comparison of MCNPx PoliMi output to point kinetics model (Equation 3.36) of photons escaping from a ${ }^{235} \mathrm{U}$ metal sphere $4 \mathrm{~cm}$ radius with $10 \mathrm{~cm}$ of polyethylene shielding. . . . . . . . . . . .

3.10 Comparison of MCNPx PoliMi output to point kinetics model (Equation 3.36) of photons escaping from a ${ }^{235} \mathrm{U}$ metal sphere $4 \mathrm{~cm}$ radius with $10 \mathrm{~cm}$ of polyethylene shielding without photon absorption in the polyethylene modeled. The lack of fit is expected due to the in-

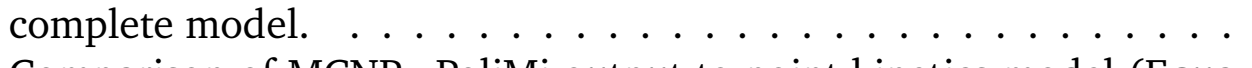

3.11 Comparison of MCNPx PoliMi output to point kinetics model (Equation 3.36) of photons escaping from a ${ }^{235} \mathrm{U}$ metal sphere $6 \mathrm{~cm}$ radius with $5 \mathrm{~cm}$ of polyethylene shielding. . . . . . . . . .

3.12 The detector setup used for simulations with 18 total $10 \times 10 \mathrm{~cm}^{2}$ detectors . . . . . . . . . . . . . . . . . .

3.13 Comparison of MCNPx PoliMi output to point kinetics model (Equation 3.46) of neutrons escaping from ${ }^{235} \mathrm{U}$ metal spheres of 1,4 and $6 \mathrm{~cm}$ in radius respectively. . . . . . . . . . . . . .

3.14 Comparison of MCNPx PoliMi output to point kinetics model (Equation 3.46) of photons escaping from ${ }^{235} \mathrm{U}$ metal spheres of 1,4 and 6 $\mathrm{cm}$ in radius respectively. . . . . . . . . . . . . . . 
3.15 Moments vs enrichment for uranium spheres of 1 , 4, and $6 \mathrm{~cm}$ respectively. . . . . . . . . . . . . . . . . . . . 9 94

3.16 Propagated perturbations (seen in Table 3.3) for the first and second factorial moments of neutron multiplets escaping a $6 \mathrm{~cm}$ uranium sphere. . . . . . . . . . . . . . . . . . . . 97

3.17 The effect of the radius, $R$, with $\pm 0.25 \mathrm{~cm}$ uncertainty propagated through to the first and second factorial moments of photon multiplets escaping a $6 \mathrm{~cm}$ uranium sphere. . . . . . . . . . . . .

3.18 All propagated perturbations (seen in Table 3.3) for the first and second factorial moments of photon multiplets escaping a $6 \mathrm{~cm}$ uranium sphere. . . . . . . . . . . . . . . . . 100

4.1 Setup of geometry with HPGe detector included. . . . . . . . . 112

4.2 The angular field of view of each pixel with respect to the source spot. 113

4.3 Correlation of the induced radiation detected in the HPGe detector following an detection in the specified alpha pixel. . . . . . . . 114

4.4 Pulse height spectra of the total alpha counts, alpha counts correlated to the HPGe detector, and correlated alpha counts with background subtracted. . . . . . . . . . . . . . . . . . . 115

4.5 Experimental setup of measurement done to determine the ability of this technique to differentiate nearby materials. . . . . . . 118

4.6 Correlated and total spectra of the measurement shown in Figure 4.5. 120

4.7 Correlated and total spectra of polyethylene shielded DU . . . . . . . 122

4.8 Correlated and total spectra of aluminum shielded DU. . . . . . . . . 124

4.9 Simulations $14 \mathrm{MeV}$ prompt activated spectra of both DU and HEU. . 126

4.10 Exploration of uranium signatures in the spectra. . . . . . . . . . . 128

4.11 Experimental setup of $\mathrm{NaI}$ detector measuring spectra from polyethylene shielded DU . . . . . . . . . . . . . . . . . . . 129

4.12 Correlation of the induced radiation detected in the HPGe detector following an detection in the alpha pixels (pixel 7 and 8 data summed).130

4.13 Pulse height spectra of the total alpha counts, alpha counts correlated to the NaI detector, and correlated alpha counts with background subtracted. . . . . . . . . . . . . . . . . . . . . 131

4.14 Experimental setup of measurement done to determine the ability of this technique to differentiate nearby materials using a NaI detector. 132

4.15 Correlated and total spectra of the measurement shown in Figure 4.14.134

4.16 Correlated and total spectra of polyethylene shielded DU. . . . . . . 136

4.17 Experimental setup to investigate the contribution from fission neutrons inducing gamma-rays. . . . . . . . . . . . . 138

4.18 The resulting spectra from the measurement shown in Figure 4.17, with and without the polyethylene block present. . . . . . . . . 139 
A-1 Vertical scan of middle of large detector with low threshold, comparison of data and simulation. . . . . . . . . . . . . . . 149

A-2 Vertical scan of middle of large detector with high threshold, comparison of data and simulation. . . . . . . . . . . . . 150

A-3 Color mapped response of detector face with high threshold, comparison of simulation (right) and data (left). Units are in counts per source detection. . . . . . . . . . . . . . . . 151

A-4 Digitizer position specific detector responses for small (top), medium (middle), and large (bottom) detectors. . . . . . . . . . . 153

A-5 Simulated position specific detector responses. . . . . . . . . . . 154

A-6 Percentage of counts below each channel plots for small (top), medium (middle), and large (bottom) detectors shows the variation of pulse heights with position. . . . . . . . . . . . . . . . 155

A-7 The small detector response represented by all spectra taken for the various locations as well as a colormap showing the variation of threshold. The red line on the plot of the spectra shows the threshold setting use in the colormap. . . . . . . . . . . . . . . 158

A-8 The medium detector response represented by all spectra taken for the various locations as well as a colormap showing the variation of threshold. The red line on the plot of the spectra shows the threshold setting use in the colormap. . . . . . . . . . . . . . . 159

A-9 The large detector response represented by all spectra taken for the various locations as well as a colormap showing the variation of threshold. The red line on the plot of the spectra shows the threshold setting use in the colormap. . . . . . . . . . . . . . . . . . . 160

A-10 The very large detector response represented by all spectra taken for the various locations as well as a colormap showing the variation of threshold. The red line on the plot of the spectra shows the threshold setting use in the colormap. . . . . . . . . . . . . . 161

A-11 Digitizer position specific detector responses for large detector, in the same style as Figure A-4.

A-12 Percentage of counts below each channel plots for small (top left), medium (top right), large (bottom left), and very large (bottom right) detectors shows the variation of pulse heights with position. . . . . . 162

A-13 Simulation of the detector response after modifying the post processor to account for position sensitivity. . . . . . . . . . . 164

B-1 Average efficiency curve for all eight detectors. . . . . . . . . . . 167

B-2 time-of-flight plot using a ${ }^{252} \mathrm{Cf}$ source, used to calibrate detectors . . 168

B-3 Gated and total pulse height spectra from the alpha pixel used in all experiments. The location of the chosen threshold is labeled with an arrow. . . . . . . . . . . . . . . . . . . . 170 
B-4 Comparison of the correlated neutron spot as imaged by NMIS (left) and the Gaussian based fit (right). Note: these two figures are not exactly equivalent, as the left one has inherent detector effects not present in the right figure. . . . . . . . . . . . . . 172

C-1 This figure illustrates the setup used to build Equation C-1 $\ldots$. . 174

E-1 The spectra (left plot) demonstrate the change of the pulse heights as the PMT gain shifts due to increased counts. The right plot shows pulse height shifts of NaI pulses over time. . . . . . . . . . . 178

E-2 A spectrum before and after being corrected for PMT gain shift. Spectra were offset from each other on the $y$-axis for visualization purposes. . . . . . . . . . . . . . . . . . . . . . . . 179 


\section{List of Tables}

2.1 Summary of results from the measurement of all DU and tungsten

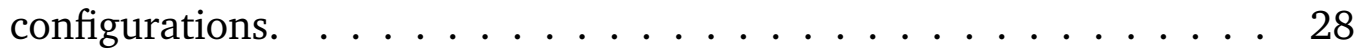

2.2 Percent difference between DU and tungsten for each signature. . . 28

2.3 Comparison of data and simulation for all measurements. . . . . . 36

2.4 Signatures of bare uranium varying in enrichment . . . . . . . . . 44

2.5 Signatures of uranium shielded by 2" of aluminum varying in enrich-

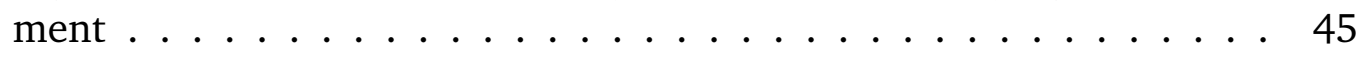

2.6 Signatures of uranium shielded by 2" of lead varying in enrichment . 45

2.7 Signatures of uranium shielded by 2 " of polyethylene varying in enrichment . . . . . . . . . . . . . . . 46 46

3.1 Point Kinetics Enrichment Prediction from Neutron Distribution . . 71

3.2 Point Kinetics Enrichment Prediction from Photon Distribution . . . . 78

3.3 Table of perturbations . . . . . . . . . . . . . . . 97

3.4 Comparison of point kinetics moments to measurements taken by NMIS101

4.1 Energies of some of the prompt gamma-rays that are likely to be seen in spectra from these measurements . . . . . . . . . 117

4.2 Integrals of the peaks of interest from spectra shown in Figure 4.6 . . 121

4.3 Integrals of the peaks of interest from spectra shown in Figures 4.7 -

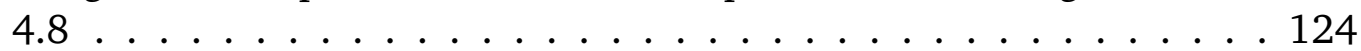

4.4 Integrals of the peaks of interest in counts per minute (CPM) from spectra shown in Figure $4.15 \ldots \ldots$. . . . . . . . . 135

4.5 Integrals of the peaks of interest in counts per minute (CPM) from spectra shown in Figure $4.16 \ldots . . \ldots 137$

A-1 Definitions of all detectors used in this research $\ldots \ldots \ldots \ldots$ 


\section{1 | Introduction}

The main goal of this project is to investigate how photon and neutron signatures from an Associated Particle Imaging (API) Deuterium-Tritium (DT) neutron generator detector system can be used to non-destructively predict the enrichment of uranium in an unknown configuration of shielded uranium. In order to nondestructively predict enrichment, two things are necessary: 1) A signature or set of signatures that varies with enrichment in a detectable way, and 2) tools that can be used to relate the signature to uranium enrichment. This project is broken up into three distinct parts that explore sets of signatures and tools for predicting enrichment of shielded uranium.

The commonality between the three parts is the use of an API-DT generator to correlate induced radiation to the known time and directional information of the source $14 \mathrm{MeV}$ neutron. Since performing neutron imaging with these devices is well established (2), it is assumed that the general geometry of an object can be attained with neutron imaging within the same detector system, and only signatures that could relate to fission are explored here. While both neutron and photon signatures are explored, the photon signatures are the main focus because these signatures are more novel, especially when related to an API detector system.

The three sets of signatures and prediction tools that are explored are the following:

1. Signature and predictive tool exploration: The correlated (source-photon, source-neutron) and bicorrelated (source-photon-photon, source-photon-neutron, 
source-neutron-neutron) detection rates, separated by their time-of-flight properties, are investigated for varying uranium enrichments and shielding types. A 3-D radiation transport Monte Carlo code is investigated to predict the signatures for varying enrichment.

2. Predictive tool exploration: Again, the correlation and bicorrelation detection rates are the signature of interest, but the predictive tool is a point kinetics technique that models a fission chain as a branching process.

3. Signature exploration: The prompt neutron-induced photons from shielding material are measured with a gamma-ray spectrometry detector to determine if this signature varies in a detectable way with enrichment.

Exploration 1 will begin by presenting results from a series of experimental measurements from an API detection system of signatures from depleted uranium shielded by different types of materials. Beginning with measurements immediately introduces the reader to the real signatures from an API detection system before moving on simulations. Comparing the data to complex 3-D radiation transport Monte Carlo simulations (MCNP-PoliMi) determines the level of confidence for the simulation tool and post processor for each type of signature. An exercise is performed to determine the level of enrichment that could be predicted using each signature, taking into account the level of agreement between the data and simulations.

Exploration 2 considers the same correlated signatures, but uses a simple point kinetics formulation in place of the physically accurate radiation transport code used in the previous exploration. Additional to exploring the usefulness of this tool, the point kinetics equations provide further insight into the correlated signatures 
by unfolding the probabilities for higher order coincidences that are not directly analyzed in the previous section.

Exploration 3 involves incorporating a gamma-ray spectrometry detector into the API detection system, which allows for investigation of the gamma-ray energy, which is not apparent in the previous two sections. A series of measurements are performed to determine if the fission neutrons induce a detectable level of gammarays in polyethylene shielding. If this signature were detectable, it may be possible to relate the number of gamma-rays detected to the number of neutrons released from an induced fission chain, which could be used to determine enrichment.

This set of explorations is not an exhaustive list of potentially useful signatures from an API detection system. However, when taken together, a fairly well rounded picture of the prompt radiation signatures detected by an API detection system begins to emerge; By the end, properties related to timing distribution, number distribution, and energy distribution are all encountered.

This dissertation will proceed by first expanding on the motivation of this work, and then presenting some background information about active interrogation and associated particle techniques. Then the three distinct explorations will be presented and discussed. The conclusions to this dissertation will discuss which signatures and techniques are promising, and where further investigation is needed. 


\subsection{Motivation}

Detection and characterization of shielded uranium is the objective of ongoing research in multiple nuclear security fields, including nuclear forensics, treaty verification, emergency response, and material control and accountability (3). The challenge of this problem is partially due to the weak radiation signals that are naturally emitted from ${ }^{235} \mathrm{U}$ decay/spontaneous fission. The highest intensity gammaray from the decay of ${ }^{235} \mathrm{U}$ is $186 \mathrm{keV}$, which is easily attenuated by shielding materials. For example, a quarter centimeter of lead shields $99 \%$ of $186 \mathrm{keV}$ gamma-rays. The spontaneous fission rate in ${ }^{235} \mathrm{U}$ is generally considered to be too low to act as a method of detection; there is only about 30 neutrons per second released from one IAEA significant quantity (25 kg) of 90\% enriched uranium (enrichment arbitrarily chosen). The solution to this detection problem for many research groups has been to use active detection methods, in which an external source is used to cause interactions in uranium which release radiation.

The different production and transport properties of photons could make them an excellent counterpart to neutrons for use in characterization of an unknown object. The neutron signature from low-Z shielded uranium is problematic because of the high scattering rate and large energy loss per scatter. For an API system, this thermalization causes the neutrons to arrive over a longer time period, which leads to the signal to background levels being reduced. This makes the neutron signatures in time-of-flight plots difficult to use.

Photons tend to travel easier through certain light materials, which are known to greatly attenuate neutrons, such as polyethylene or water. Heavy, dense materials such as lead or uranium, which greatly attenuate photons, are typically not problematic for neutrons. The proposed work will investigate the gamma-ray signa- 
tures from shielded uranium configurations to determine if incorporating gammaray analysis could be a useful counterpart to neutron signatures.

\subsubsection{Applications of Proposed Work}

The goal of this work is to allow for the detection and characterization capabilities of API DT neutron imaging systems to be more versatile. The inclusion of source correlated and bicorrelated photon detection rates might allow for characterization in a wider range of shielding types.

For many security applications, these enhancements to the system would be highly beneficial. Even if the enrichment measurements are not highly precise, they would still potentially be able to verify the presence or absence of special nuclear material (SNM), or differentiate between DU and HEU.

The potential for a relatively transportable system that could characterize an unknown object within a short measurement time would be useful in the nuclear forensics field. The need for fast characterization of pre-detonation material is one of the goals of nuclear forensics, and the fast, non-destructive characterization of highly enriched uranium (HEU) is a difficult problem. While nuclear forensics primarily consists of destructive analysis techniques, if an item needs to be sent to a laboratory for forensics analysis, an initial nondestructive characterization would greatly aid in knowing how to send the item and which laboratory to send the item to (4). 


\section{$1.2 \quad$ Background}

\subsubsection{Active Detection Techniques}

Active interrogation is a technique that uses a radiation source to induce interactions in a target object, followed by the detection of the induced radiation. Active interrogation typically either uses a neutron source or a high energy photon source, though other source types have been studied, such as high energy protons or muons. (5)

Active interrogation is known to be a promising solution for the difficult problem of detection and characterization of shielded uranium configurations (5). Research involving active interrogation techniques are especially prevalent in detection of HEU in cargo containers $(6 ; 7)$, nuclear fuel characterization $(8 ; 9)$, and arms control and treaty verification $(1 ; 10)$. In many applications, the goal is to detect the presence of SNM, usually in heavily shielded assemblies. In other applications, the goal is to characterize the SNM, particularly determining the total mass, isotopic content, or multiplication properties. (5)

In nearly all fields involving the nondestructive detection and analysis of SNM (including passive and active techniques), the main challenge is the attenuation of the signal in comparison to the background rate. While active interrogation increases the signal output of an assembly, it also causes a higher background rate and creates additional challenges inherent to these more complex systems. Runkle et. al. (5) summarizes the challenges of active detection well:

Active interrogation provides a means of increasing the intensity of emitted radiation when shielding is an otherwise limiting factor. The implementation of active interrogation techniques, however, comes at a cost 
not only in terms of finance and dose to inspected objects but also in terms of induced backgrounds, system complexity, and extended acquisition times.

\subsubsection{Neutron Interrogation}

Neutron sources used for active interrogation include x-ray sources coupled to a material with a high $(\gamma, \mathrm{xn})$ cross section such as beryllium or deuterium (11); radioisotope neutron sources, such as $C f^{252}$ (12); and electronic neutron generators (ENGs) which accelerate light ions to cause fusion reactions (2). The goal in many neutron interrogation methods for nuclear security is to induce fission and then identify characteristic fission signatures. Most sources are high energy neutron sources, which have the advantage of being very penetrating. Portable, sealed tube accelerators utilizing fusion of deuterium-deuterium (2.5 Mev neutrons, $10^{6}-10^{8}$ neutrons/s yields) or the deuterium-tritium $\left(14.1 \mathrm{MeV}\right.$ neutrons, $10^{8}-10^{10}$ neutrons/s yields) are most frequently used (5).

The benefits of using neutron sources over photon sources include the option of portability and the highly penetrating nature of neutrons through high-Z material. The disadvantages of using neutron sources over photon sources include the neutron attenuation in the presence of low- $\mathrm{Z}$ materials and the potential of activating materials, introducing more background into the system.

\subsubsection{Photon Interrogation}

Photon sources used for active interrogation most often use electron accelerators directed at a target to produce bremsstrahlung radiation. To achieve bremsstrahlung radiation with high enough energy to produce photofission interactions, the electron energy must be at least $6 \mathrm{MeV}$, but accelerators usually operate 
at 9-10 MeV $(5 ; 13)$. Some research also uses other types of photon sources, such as proton beam based photon sources (14).

Nuclear Resonance Fluorescence (NRF) is a technique that utilizes a photon source that excites the material which then de-excites via characteristic gammarays. Spectroscopy can then be used to determine the isotopic content of the material. Gamma-rays from NRF are a prompt signal that are typically an order of magnitude higher than decay gamma-rays of SNM and have energies higher than prompt gamma-rays from fission. However, interrogating shielded materials requires a high intensity source to penetrate the shielding, and the signature can suffer from interference and self attenuation which degrade the signal to background rate. $(5 ; 10)$

The benefits of photon sources is their ability to penetrate low $\mathrm{Z}$ material, which is commonly found in shipping compartments. However, the background levels can limit the types of signals that are available for analysis (5).

\subsubsection{Types of Signals Analyzed}

Active interrogation techniques for detection of SNM typically use fission signatures such as the number, energy, or timing distribution of radiation emitted from fission. The fission signatures used for analysis can be categorized into prompt, die-away, and delayed neutron and gamma-ray signatures, though the dieaway signature is considered to be a type of prompt signature $(5 ; 15 ; 16)$. The prompt radiation is defined to be any radiaton emitted by the fission interaction itself (as opposed to decay products). In experimental terms, the prompt signature is generally defined as the radiation released within $10 \mathrm{~ns}$ of a fission event (17). The die-away signal looks at the decay of the prompt neutron signal as the source and fission neutrons thermalize within the system and are slowly detected up to 
several miliseconds after the source neutrons have been stopped. When no fissioning is present in the material the neutron rate will drop off sharply, but fissioning will cause a steady decrease (die-away) of the neutrons after the pulse has ended (16). Delayed neutron and gamma-ray signatures are released from the decay of the fission products, and are generally divided into several half life groups. The time range defined for the delayed radiation varies, but is generally in the range of about 0.1 to 100 seconds $(5 ; 15)$.

Most work involving photon interrogation uses a pulsed bremsstrahlung interrogation source and detects delayed neutron and photon signatures (18), however research involving prompt signatures does exist. Photon interrogation techniques using prompt fission signatures of photons or neutrons are difficult because of the intensity of the source and induced background interference (5). The other primary technique involving photon interrogation is NRF, which doesn't involve fission signatures (10).

Neutron interrogation techniques most often involve pulsed DT-ENGs with pulse widths of $10-100 \mu \mathrm{s}$ at frequencies of $10-1000 \mathrm{~Hz}$, utilizing the die-away signature between pulses (5). Other techniques involve using the delayed neutrons and gamma-rays to detect lightly shielded HEU (7). The type of system that is typically used for prompt radiation analysis is an associated particle imaging (API) DT or DD neutron generator (19), discussed in more detail in the next section. The prompt radiation is associated with such high background rates that many systems are set up for detection in the die-away or delayed time region for analysis (5).

Much of the work in active interrogation involves applications that only require the presence of SNM to be identified, such as cargo inspection. However, detection and characterization of SNM in an inspection object is often required, which typically requires more complex equipment or analysis techniques. Systems meant 
to characterize SNM will have capabilities such as object imaging (2), gamma-ray spectroscopy (20), coincidence counting (21), etc. Nuclear security fields that utilize active interrogation, such as nuclear forensics (22), disarmament treaty verification (21), and spent fuel characterization (8), require more sophisticated analyses of unknown configurations.

\subsubsection{Associated Particle Neutron Detection}

The associated particle technique has been used since the 1950's (23), but has evolved substantially as computing capabilities have continuously developed to allow for faster processing speed, which now allows for time-of-flight measurements with less than nanosecond timing resolution. Associated particle sealed-tube neutron generators (APSTNG or sometimes APNG) have been developed for treaty verification and nonproliferation applications since the early 1990's at Los Alamos National Laboratory (LANL) (19), and for the most part they have continued to be used in those fields. APNG systems allow for source neutrons (or particles induced from source neutron interactions) to be time-tagged by the associated alpha particles from the DT fusion interactions. Using this small correlation window initiated by the source event allows for a large reduction in background interference. Associated particle imaging (API) technique uses a pixelated alpha detector to timeand directionally-tag radiation, and it is most often used in imaging technology, including some advanced PET medical imaging systems.

\subsubsection{Associated Particle Imaging Research}

Several groups around the world use APSTNG devices for SNM detection and characterization. The Nuclear Material Detection and Characterization group at 
Oak Ridge National Laboratory (ORNL) has been working for many years on a variety of neutron imaging systems that utilize API DT neutron generators, such as the Nuclear Materials Identification System (NMIS) and the Advanced Portable Neutron Imaging System (APNIS) (2). Lawrence Berkeley Laboratory and University of California in Berkeley also do some imaging work with API DT neutron generators (24), but the work is focused on improvements of the neutron generator instrumentation itself. The Purdue University Applied Physics Department also contributes to APSTNG related research in the U.S., mostly involving the identification of both nuclear and non-nuclear threats, such as chemical and biological warfare agents and explosives $(25 ; 26)$. Other U.S. national laboratories are at times involved in simulations or collaborations involving APSTNG devices $(27 ; 28)$, but ORNL remains the leader in this research area in the U.S.

Outside of the U.S., the main associated particle technique research is done by a few Russian institutions and those involved in the EURITRACK project. Kuznetsov et. al. (29) from the V. G. Khlopin Radium Institute in Russia utilize API DT neutron generators to detect heavily shielded HEU or for identifying non-nuclear explosive device components. Bystritsky et. al. (30) from the Joint Institute for Nuclear Research in Russia and the Federal Security Service of the Russian Federation are also using API DT neutron generators to identify non-nuclear explosives.

EURITRACK (20) is a project which a few European countries are involved in with the goal of making shipping ports more secure. This research also involves API DT neutron generators for identification of both nuclear and non-nuclear illicit materials.

The main disadvantage of systems that utilize API technique is the complexity of the instrumentation and analysis, especially when the complexity of the system leads to long measurement and analysis times and high costs. However, the problem 
of characterization of shielded uranium is a difficult one, and may justify this level of complexity.

\subsubsection{The Nuclear Materials Identification System}

It is judicious to describe NMIS in more detail, since this dissertation work was done at ORNL with access to NMIS equipment.

NMIS has been under development since 1984, and has been shown to be capable of tomographic imaging of a variety of configurations, verification of fissile material via fission mapping, mass and enrichment measurements of certain types of objects, and identification of many types of materials. $(2 ; 31 ; 32)$

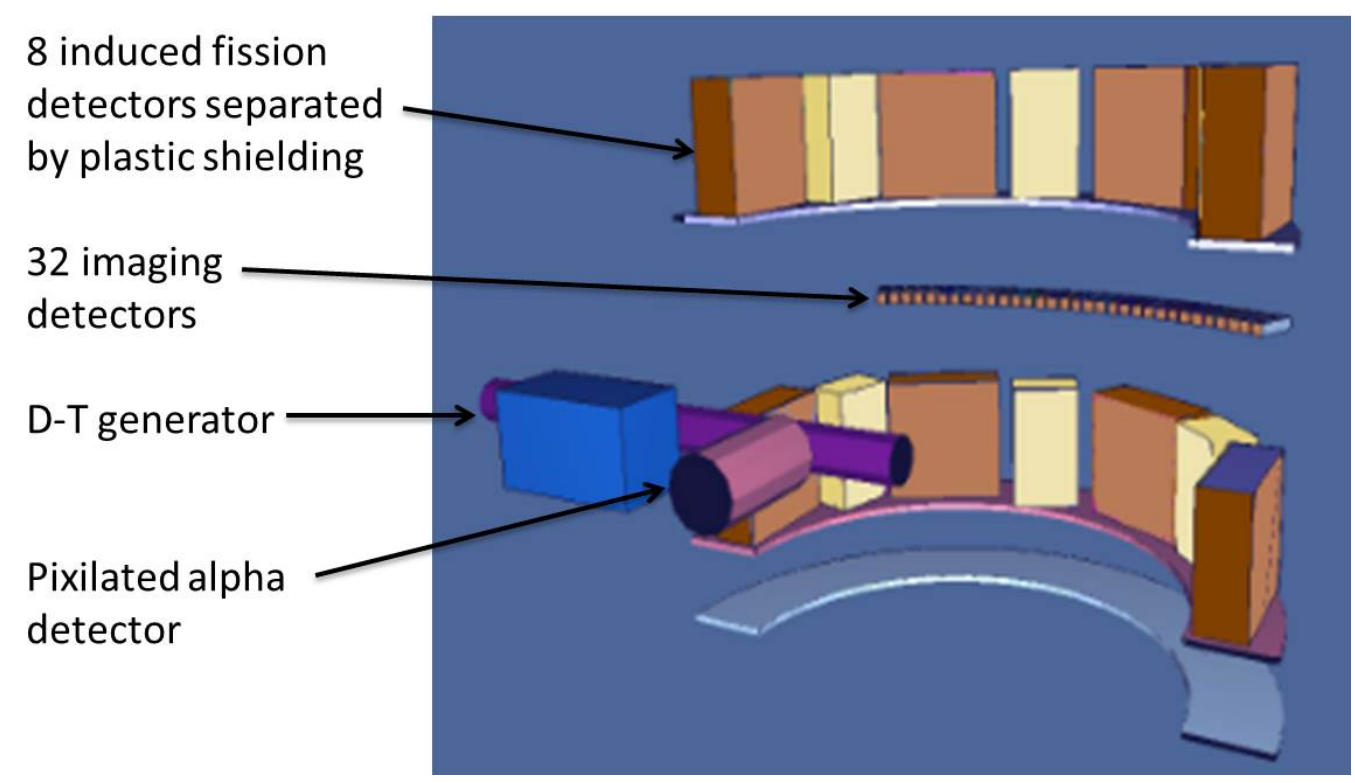

Figure 1.1: Nuclear Material Identification System (1)

NMIS in its most current form consists of a Thermo Scientific API 120 neutron generator with a Hamamatsu H9500 position sensitive PMT mounted to the alpha detector, which allows for time- and directional- tagging of the $14 \mathrm{MeV}$ source neutrons. The pixelated PMT divides the source correlated detector view into a 
horizontal fan of 16 directions. The system utilizes $321 " \times 1 " \times 4$ " plastic detectors arranged in an arc for transmission imaging, and $810.6 " \times 10.6 " \times 3.7$ " plastic detectors above and below this detector arc, used for detecting and mapping the locations of fission neutrons. The system of transmission detectors and neutron generator are capable of scanning up and down in order to create a 3-D image from these cross sectional images. The transmission detector array also shifts slightly radially back and forth to attain better spatial resolution. This configuration can be seen in Figure 1.1.

For data aquisition, NMIS consists of two acquisition boards capable of processing each of the 5 available channels at rates up to $1 \mathrm{GHz}$, though two channels are used for synchronizing the boards. The fast plastic detectors and high processor speed allows for collection of source correlated timing data with high enough timing precision to differentiate between fission gamma-rays, $14 \mathrm{MeV}$ transmission neutrons, and fission neutrons based on their arrival times at the detectors, an example of which can be seen in Figure 1.2. A more detailed description of NMIS is given by Crye (33).

An example cross sectional image taken by NMIS can be seen in Figure 1.3. A representation of the actual image is shown in the top right, the transmission image taken by NMIS is shown in the top left, and the fission map (created by the bicorrelation detection rate) is shown in the bottom left. The bottom right image combines the transmission image with the fission map in order to locate and quantify the fissionable material.

There is also a fieldable version of NMIS, called FNMIS, under development which will incorporate gamma-ray spectroscopy (31). 


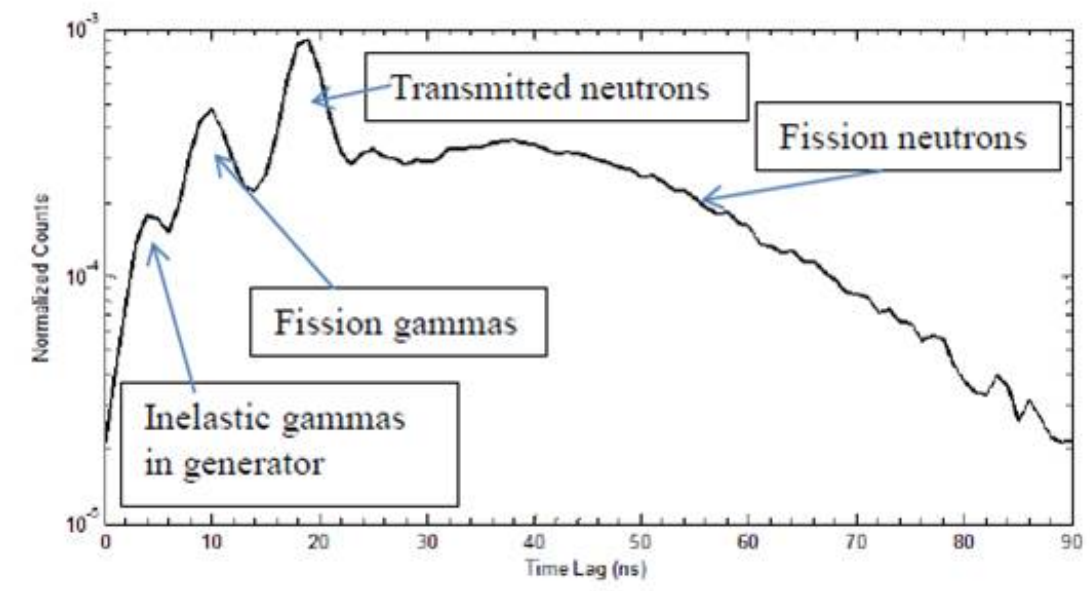

Figure 1.2: Source Correlated time-of-flight (2)

\subsubsection{Access to Materials}

The uranium sample used in most simulations is a hollow cylinder of $18 \mathrm{~kg}$ of uranium metal, which is simulated at a variety of enrichments. When shielding is added, it is modeled as a larger cylinder, in which the shielding surrounds the uranium casting, but does not cover the top or the bottom. This object is based on two real inspection objects, one is depleted uranium (DU) and the other is HEU (93\% ${ }^{235} U$ and $5 \%{ }^{238} U$, as well as small amounts of ${ }^{234} U$ and ${ }^{236} U$ ). The DU object is located at ORNL and was avaible for measurements, but the HEU test object is located at Y-12 and has been used for measurement campaigns in the past (34). There was also a tungsten casting of the same dimensions available which was used for measurements taken in Chapter 2. 

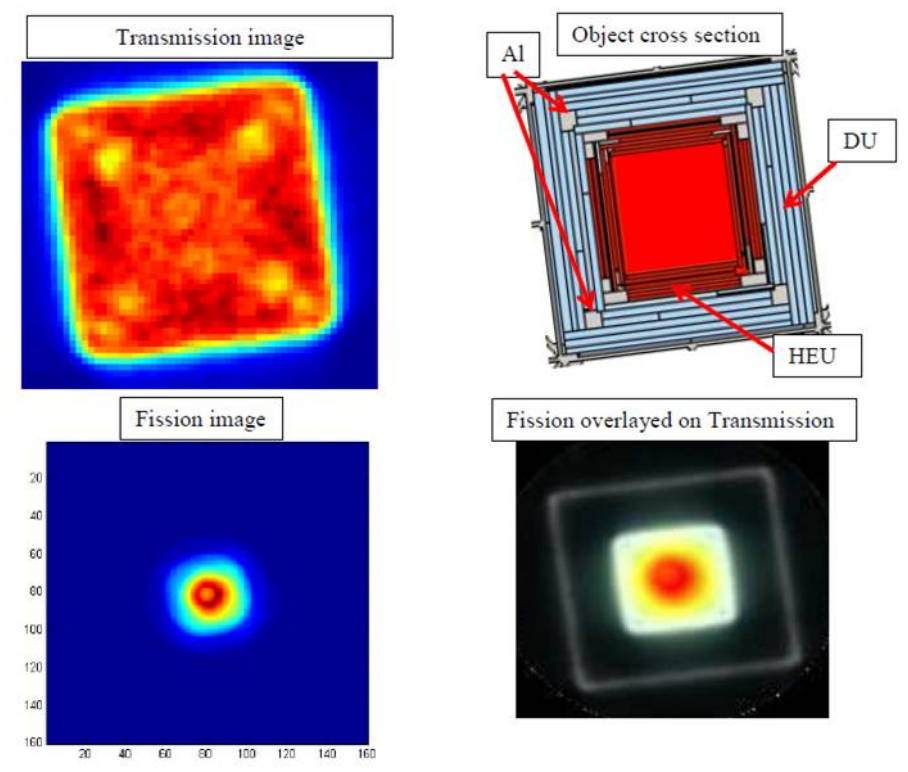

Figure 1.3: Cross Sectional Image Created by NMIS (2) 


\section{2 | Time-of-Flight Signatures}

\subsection{Introduction}

The main goal of this project is to investigate signatures from an API-DT generator system and analysis techniques that can be used to predict enrichment of a configuration of shielded uranium.

This chapter will investigate a set of correlation signatures using experimental data taken by an API system to determine how well they evidence fission interactions. Then a full 3-D transport Monte Carlo simulation tool will be used to predict how these signatures vary with enrichment for various configurations. In the end, conclusions will be drawn about this set of signatures and tools usefulness in predicting uranium enrichment.

Several signatures, sorted by their time-of-flight properties, will be investigated to determine if they vary strongly enough with enrichment to be useful, even when shielded by different types of material. The signatures investigated are the following:

- source-photon,

- source-neutron,

- source-photon-photon,

- source-photon-neutron, and 
- source-neutron-neutron

Higher correlation signatures generally do not have detection rates high enough to be plausible. For example, a detector system with $10 \%$ efficiency would only have a $1 \%$ efficiency for second order induced correlations (e.g. source-photonphoton) and only $0.1 \%$ efficiency for a third order induced correlation (e.g. sourcephoton-photon-photon). Shielding is an additional factor that affects the signature detectability. The shielding materials chosen were lead, an excellent photon attenuator; aluminum, an inelastic scattering gamma-ray producer, and polyethylene, a neutron moderator.

After a brief review of relevant previous research, this investigation will begin by describing a set of experimental measurements performed by the NMIS equipment described in the previous chapter. Introducing these signatures with experimental data rather than simulations or theory informs the reader about the true characteristics of these signatures. By comparing some nuclear material (depleted uranium) and non-nuclear material (tungsten), some immediate conclusions can be drawn about how useful these signatures are to evidence fissioning material, even in the presence of varying shielding materials.

Next, results from 3-D radiation transport Monte Carlo simulations of these measurements are compared to the experimental data to determine the level of agreement between data and simulations. This informs about the level of confidence that can be expected in our tools for relating signatures to enrichment. Since there was no access to enriched uranium for this project, the simulations will be used to explore how the signatures vary with enrichment. Conclusions about the ability of this set of signatures and tools to predict enrichment will be drawn based on a simple study related to the ability to predict enrichment with each signature in 
the presence of varying shielding types, taking into account the capability of the simulation to accurately represent the measured data.

\subsection{Previous Work}

Since this work most directly follows from previous work done with NMIS, the review will be limited to previous work of NMIS enrichment related studies. A. Swift's Master's thesis involved a blind study simulating the use of NMIS to characterize an unknown object. Data was simulated in MCNP PoliMi of an NMIS measurement involving imaging and fission mapping of a cylinder of HEU surrounded by a layer of DU, followed by a layer of polyethylene. The results were positive, using the image to create simulations of the object, and then varying the enrichment of the inner uranium layer until the source-neutron time-of-flight signature most closely matched the data she was given. Using this method she was able to predict the enrichment to within less than $6 \%$ of the true value.

In contrast to this, Mullens et al. (35) presented data taken with the NMIS system of HEU surrounded by polyethylene, showing the source-neutron signature to be skewed by the polyethylene and stating that the source-neutron-neutron data was too weak to be usable. While Swift's thesis showed the promise of this technique, comparing the varied enrichment simulations to measured data, as opposed to the simulated data used in her blind study, likely leads to further challenges.

The most thorough study of using MCNP-PoliMi simulations of the NMIS system to predict enrichment of an unknown uranium configuration is presented in J. Crye's dissertation (34). He took measurements at Y-12 of an HEU casting surrounded by varying levels of DU shielding, and used a similar type of technique described in A. Swift's thesis to show how enrichment could be predicted using the 
time-of-flight signatures. However, a ${ }^{252} \mathrm{Cf}$ source was placed in the center of the HEU casting and used to interrogate the HEU. In addition to the source-detector signature, he also looked at the source-detector-detector rate and the Feynman distribution, which involved both of these signatures. The term "detector" is used in place of "neutron" or "photon" here because the ${ }^{252} \mathrm{Cf}$ source was too close to the object to differentiate photons and neutrons based on time-of-flight. Another major difference was the use of small detectors $(1 " \times 1 " \times 4 ")$ instead of the typical large detectors $\left(10.6 " \times 10.6 " \times 3.7^{\prime \prime}\right)$ used in NMIS, partially due to poor agreement between experimental and simulated time-of-flight plots that was not resolved. Using this setup, he was able to predict enrichment well, predicting between $82-95 \%$ for various configurations of the $93 \%$ enriched HEU casting surrounded by different amounts of DU shielding.

From these studies, it is clear that the neutron time-of-flight signatures vary in such a way that they are a good indicator of enrichment. However, modeling the measurements made by a complicated system such as NMIS closely enough to predict enrichment is a challenge. With this in mind, this work involved some characterization of the detectors in order to more closely model the large detectors. This

is described in Appendix A. The focus of the work was a generalized exploration of the time-of-flight signatures, especially in regard to a variety of shielding types.

\subsection{Experimental Setup}

A series of measurements was done using parts of NMIS. A Hamamatsu H9500 pixelated alpha detector coupled to an API-120 DT generator was mounted onto the NMIS frame, then carefully leveled. Pixel 8 out of the total 16 pixels was used for all measurements. No other pixels were used for simplicity. The fission de- 
tector rack, as described in (34), was placed in front of the DT generator, with a total of 8 large plastic scintillators $(10.6 " \times 10.6 " \times 3.7 ")$ placed on two levels of the semicircular rack with polyethylene blocks between them to reduce cross talk. Two aluminum boxes were stacked for the inspection objects to be placed on. The DT generator was left at a height such that the source spot was 27.3 " off of the ground. This height corresponds to the center of the fission detector rack. Each inspection objects was placed such that the neutron cone would hit the middle of the object. Each inspection objects was placed 17.3" away from the source spot (source to object center distance), and in the center of the semicircular detector rack, such that the radial distance from the object center to each detector was the same. The radius of the semicircular rack was 19", and the center to center distance between the object and detectors was 22.2". This configuration can be seen in Figure 2.1.

A detailed description of the analog electronics used in NMIS are detailed in J. Crye's dissertation (34) and will not be repeated here. A description of the calibrations required to setup the pixelated alpha detector and fission detectors, as well as the measurements done for modeling purposes, are all described in Appendix B.

\subsubsection{Measurement Series}

The measurement series for this section was comprised of 14 total measurements. The measurements varied between using the DU casting and the tungsten casting in the center, as well as the type and amount of shielding used. The list below summarizes the measurements done.

$\rightarrow$ Bare DU

$\rightarrow$ DU +1 " of polyethylene 


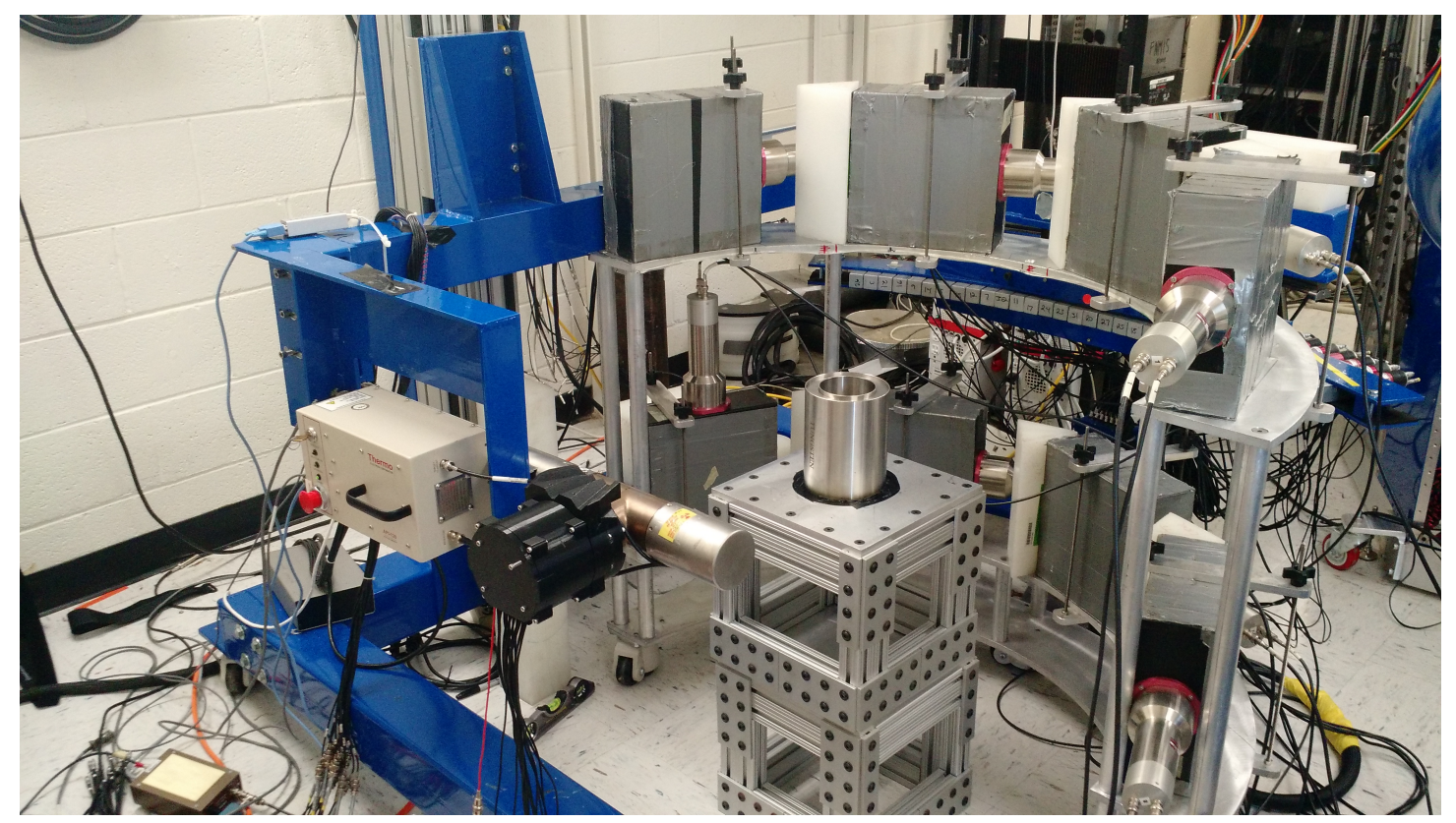

Figure 2.1: Experimental setup used for measurement series, shown with the tungsten casting.

$$
\rightarrow \mathrm{DU}+2^{\prime \prime} \text { of polyethylene }
$$

$\rightarrow \mathrm{DU}+1$ " of aluminum

$\rightarrow \mathrm{DU}+2^{\prime \prime}$ of aluminum

$\rightarrow \mathrm{DU}+1$ " of lead

$\rightarrow \mathrm{DU}+2^{\prime \prime}$ of lead

$\rightarrow$ Bare tungsten

$\rightarrow$ tungsten +1 " polyethylene

$\rightarrow$ tungsten +2 " polyethylene

$\rightarrow$ tungsten +1 " of aluminum

$\rightarrow$ tungsten +2 " of aluminum 
$\rightarrow$ tungsten +1 " of lead

$\rightarrow$ tungsten +2 " of lead

\subsection{Analysis of Experimental Data}

After the measurements were taken, the data from each measurement was sorted by correlations with an ORNL post processing program*. This program determines the correlations of all detectors with relation to the source associated particle detections, based on a variety of input parameters (e.g. time window, detector combinations, etc.).

The source-photon and source-neutron signatures are determined from the timeof-flight output of this program. The time-of-flight for the bare DU casting is shown in the upper left plot of Figure 2.2. For each plot, the total counts are normalized by the total source detections. The background levels, which are the correlation rates around $100 \mathrm{~ns}$, are subtracted from the total spectrum. The distance between the inspection object and the detectors represents a compromise between high geometric efficiency and the ability to deconvolve the types and energies of the detected radiation; the photons, $14 \mathrm{MeV}$ neutrons, and fission neutrons, are clearly distinguishable but the peaks are not well separated. Therefore some effort was spent deconvolving the peaks. The first small peak is due to photons released from the source event itself, followed by the photon peak, the $14 \mathrm{MeV}$ neutron peak, and the fission energy neutrons, which follow in a broad peak. The photons from the source are fitted and subtracted out of the spectrum. The $14 \mathrm{MeV}$ peak is also fitted by a Gaussian peak around the main peak, and a small additional Gaussian peak to account for slower scattered neutrons, which can be seen in the top plot of Figure

*written by Seth McConchie 


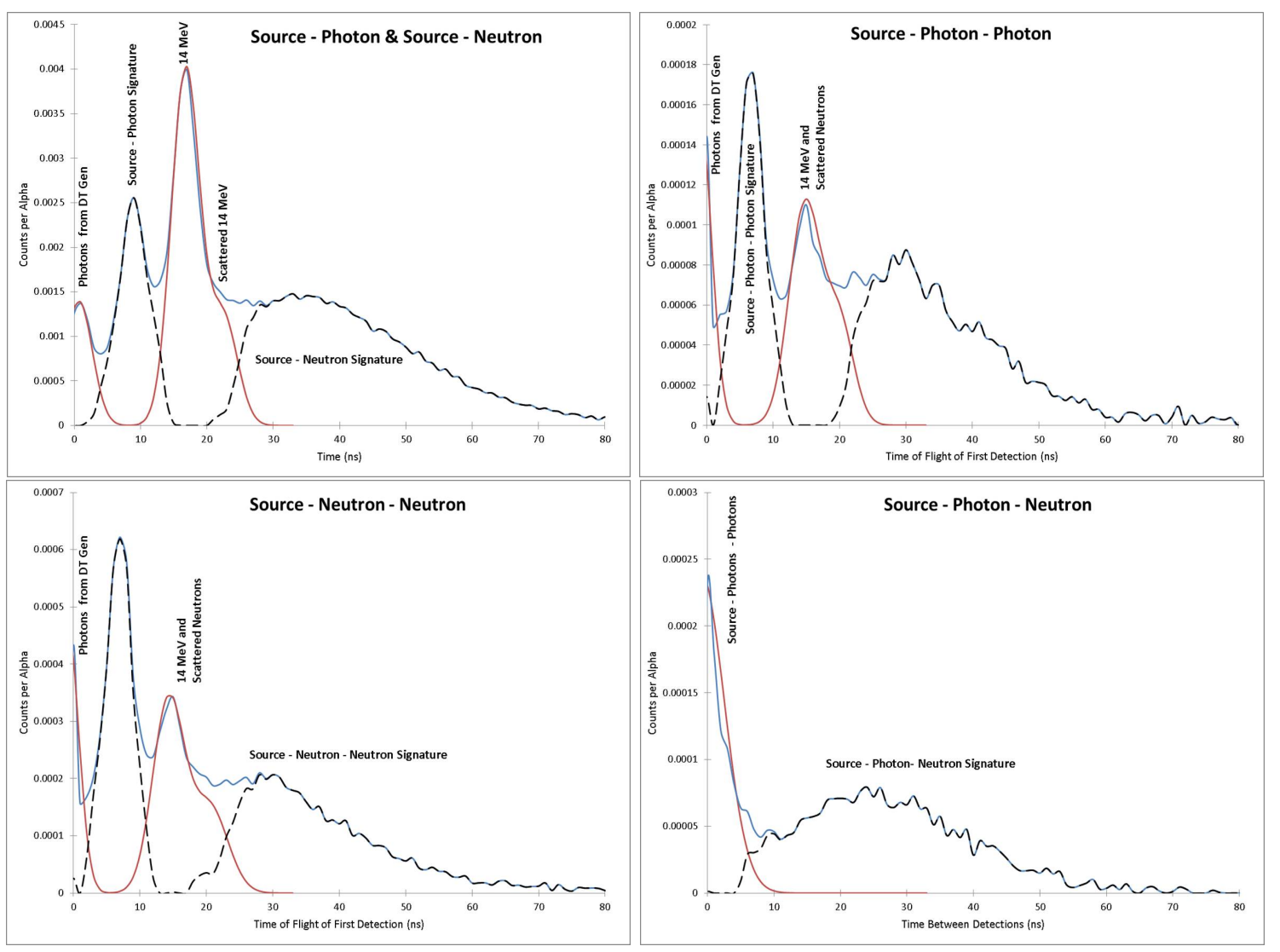

Figure 2.2: This series of plots shows how the signatures are deconvolved and what area of the spectrum is integrated to determine each signature rate. In each plot, the blue line is the data, source normalized and background subtracted; the red line is the sum of the fitted peaks to be subtracted out of the spectrum; and the black dashed line is the spectrum with the interfering peaks subtracted out.

2.2. After these signatures are subtracted out of the time-of-flight, the photon and neutron peaks are well separated, allowing the source-photon and source-neutron rates to be determined by integrating the appropriate areas of the spectrum.

The source-photon-photon, source-photon-neutron, and source-neutron-neutron signatures are separated by using two correlation types: the time-of-flight of the first particle detected after the source, and the time between the detection of the two particles. Figure 2.3 shows the separation of these signatures via these two correlations types. 


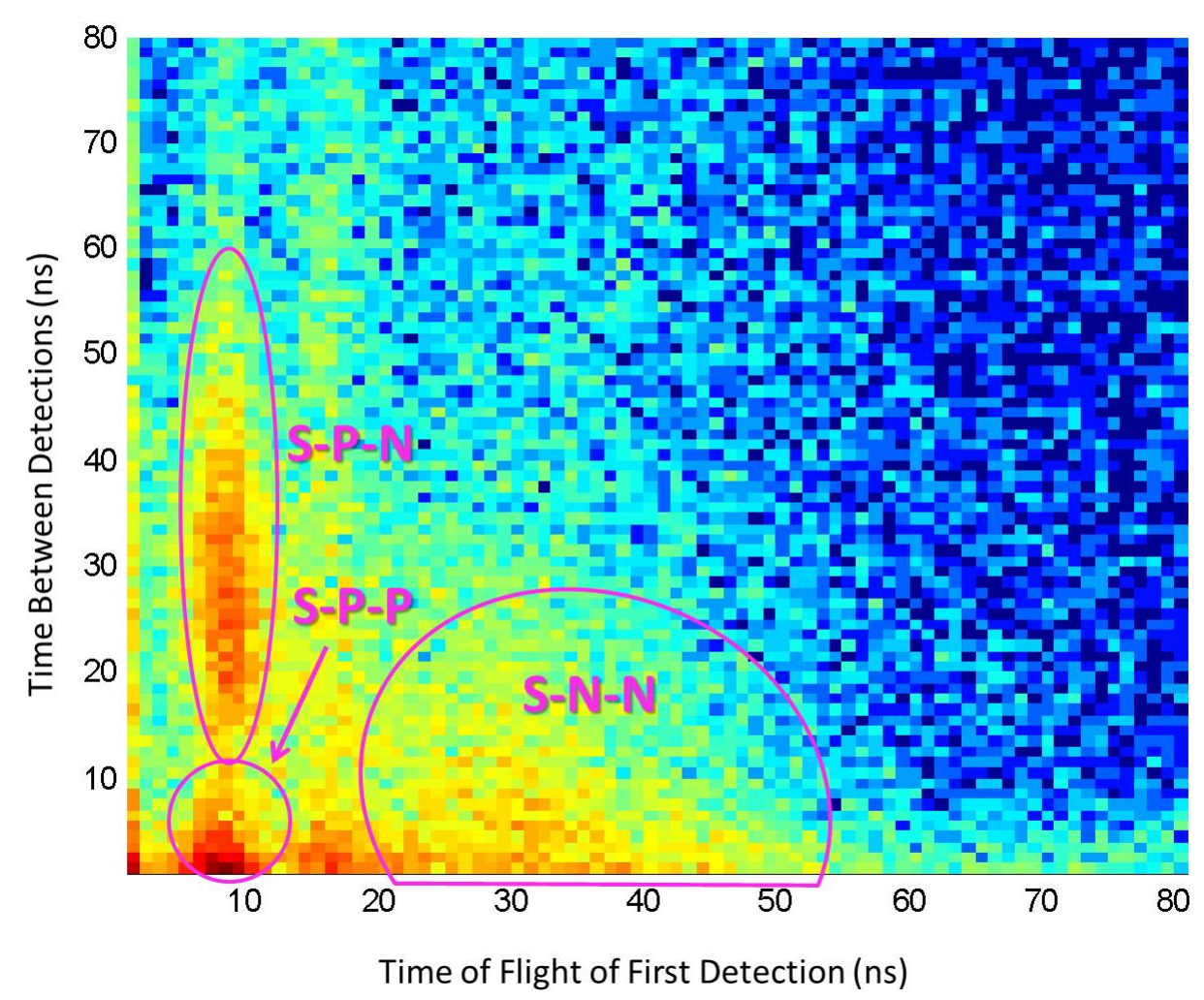

Figure 2.3: Bicorrelation plot, with source-photon-photon, source-neutron-neutron, and source-photon-neutron signatures labeled.

To determine the source-photon-photon rate, Figure 2.3 is summed along the "Time Between Detections" axis for times 0 to 12 ns. This can be seen in the upper right plot in Figure 2.2, along with the scattered neutron fit that is subtracted out. Similarly, the source-neutron-neutron rate seen in Figure 2.3 is summed along the "Time Between Detections" axis for times 0 to $70 \mathrm{~ns}$, and the scattered neutron fit is subtracted out, which can be seen in the third plot of Figure 2.2. For the sourcephoton-neutron rate, the summation is along the "time-of-flight of First Detection" axis from 1 to $11 \mathrm{~ns}$, and the source-photon-photon peak is subtracted out of the spectrum, as can be seen in bottom plot of Figure 2.2.

For all signatures, the statistical uncertainty was determined with Poisson statis- 
tics, assuming the uncertainty is the square root of the total counts, and adding in quadrature the uncertainty from the total signature and all background subtracted. It is recognized that this is a simplistic determination of uncertainty, especially when comparing to the signatures predicted by the simulations. A rigorous uncertainty determination would consider counting statistics, source uncertainty, timing uncertainty, correlated neutron cone uncertainty, etc. When combined with the imaging capabilities, a significant uncertainty contribution would be from the geometry determination via imaging. A rigorous uncertainty determination for this system has not been developed, would require significant effort, and is not within the scope of this project.

\subsubsection{Exploration of Signatures in Data}

For a signature to be useful to inform about enrichment, it must be able to differentiate a nuclear and non-nuclear material, even for highly subcritical nuclear materials. This is the rational behind using the tungsten casting, which is the same geometry as the DU casting with similar density (the density of tungsten is $17 \mathrm{~g} / \mathrm{cc}$ versus that of DU which is $18.75 \mathrm{~g} / \mathrm{cc}$ ). If a signature is useful, it should be able to distinguish even the highly subcritical depleted uranium from the benign tungsten.

Figure 2.4 shows all the signatures of interest plotted for both DU and tungsten with no shielding material present. The source-photon signature is especially interesting because it is nearly identical between DU and tungsten. This might

appear to imply that there is no significant photon fission signature escaping the uranium. However, the source-photon-photon signature is significantly different. This suggests that there are similar photon inducing interactions in the tungsten and uranium, but the photon multiplets released from uranium are higher, making 

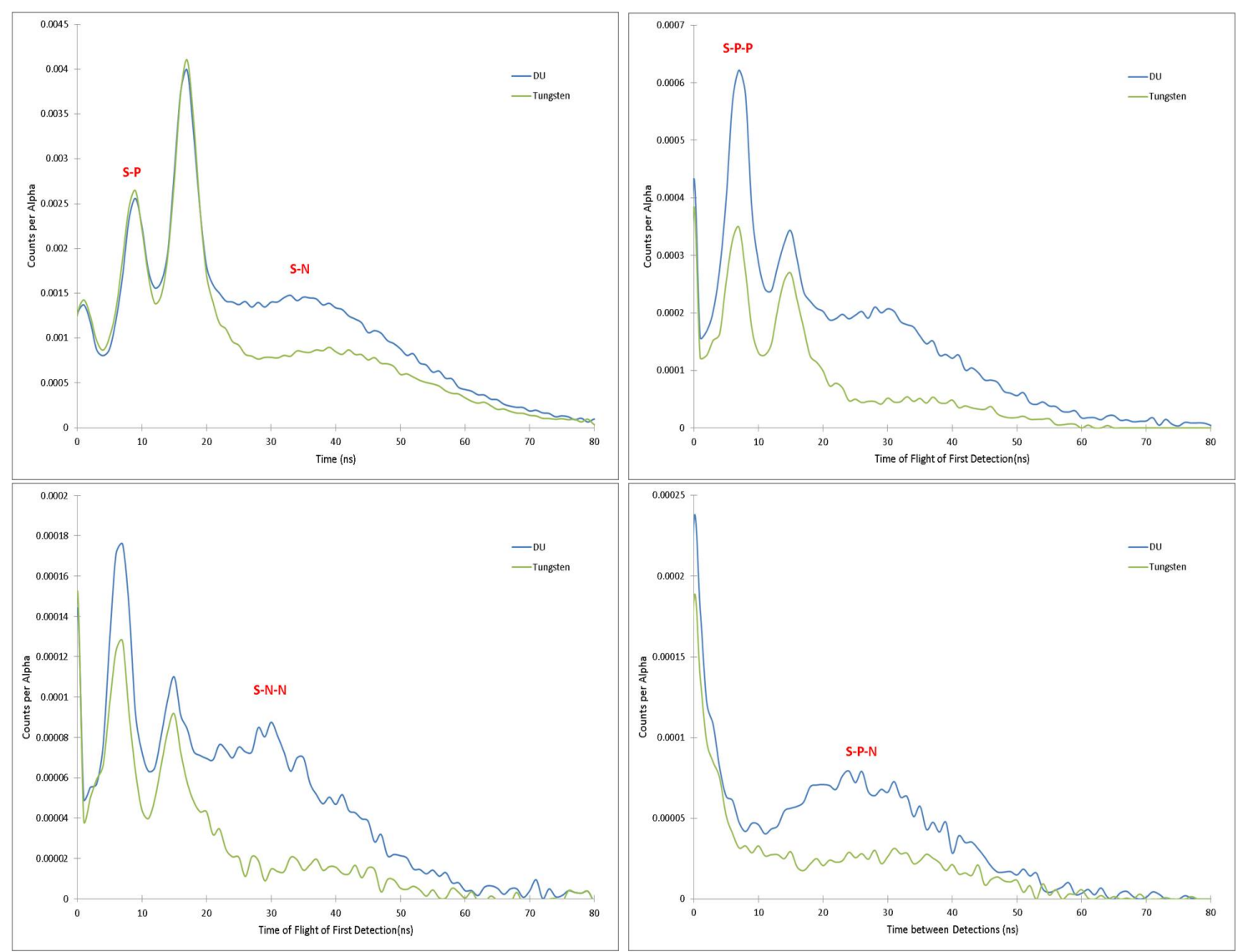

Figure 2.4: A comparison of DU and tungsten for all signatures.

the likelihood for detecting pairs of photons higher. Taking a quick look at the cross sections, this makes sense: the $14 \mathrm{MeV}$ non-elastic cross section is $0.152 \mathrm{~cm}^{-1}$ for tungsten and $0.147 \mathrm{~cm}^{-1}$ for DU, with $37 \%$ of the non-elastic cross section for ${ }^{238} \mathrm{U}$ being fission, which matches well with these results. (36)

It is clear from the source-neutron signature of tungsten that there is a significant $(\mathrm{n}, 2 \mathrm{n})$ interaction rate in the material. However, despite this, the signatures are very clearly distinguishable. Similar to the photon signature, the difference in bicorrelation signature rates is more significant.

The source-photon-neutron signature is an especially interesting signature to look at because it is highly resistant to cross talk interference (37), and because 
the difference between DU and tungsten is significant. Overall, there is a clear and significant difference between the DU and tungsten in most signatures.

Table 2.1 gives a summary of the signature rates, given in counts per alpha, for all measurements done. While these count rates appear to be very low, the alpha rate was around 1800 counts per second, so that even the weakest source-photonphoton signature built at nearly 1 count above background per second. Since these measurements were all taken for 30 minutes, long enough to build over 3 million alpha counts, the statistical uncertainty is very low.

Table 2.2 gives the percent difference between the DU and tungsten measurements, including all variations of shielding. Additionally, the time-of-flight plots for all signatures from DU influenced by varying levels of shileding can be seen in Figures 2.5-2.7. As could be guessed based on Figure 2.4, the source-photon peak is nearly the same for all measurements. As discussed previously, the source-photonphoton rate is significantly higher due to higher photon multiplets from fission than from inelastic scattering.

The largest difference between DU and tungsten across all measurements is in the neutron related signatures. The source-neutron rate is significantly different for all shielding types. This difference is amplified in the source-neutron-neutron rate, which is the highest percent difference between DU and tungsten for all measurements. The source-photon-neutron also has a large percent difference across all measurements, and has the added benefit of resistance to cross talk. 

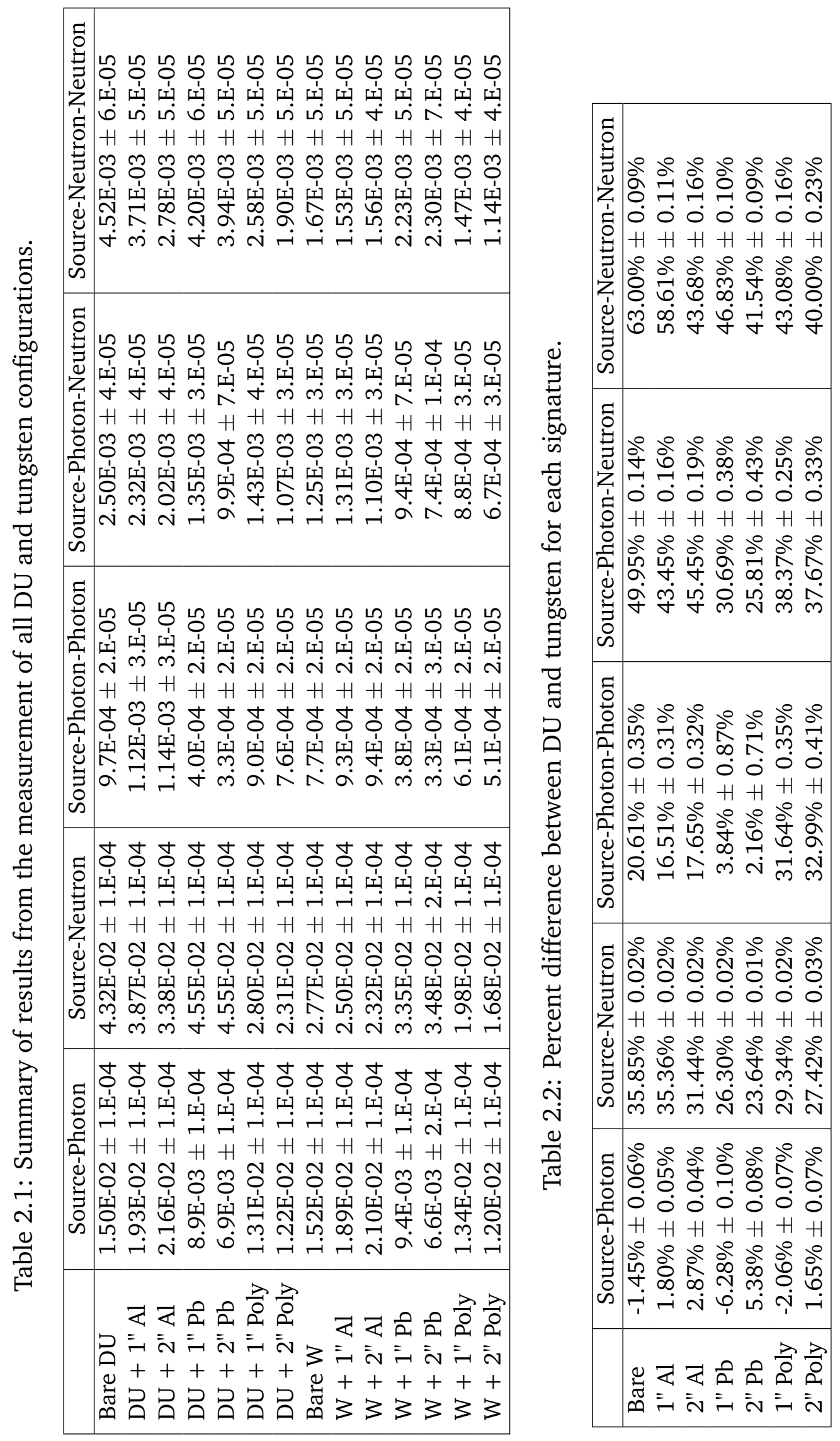

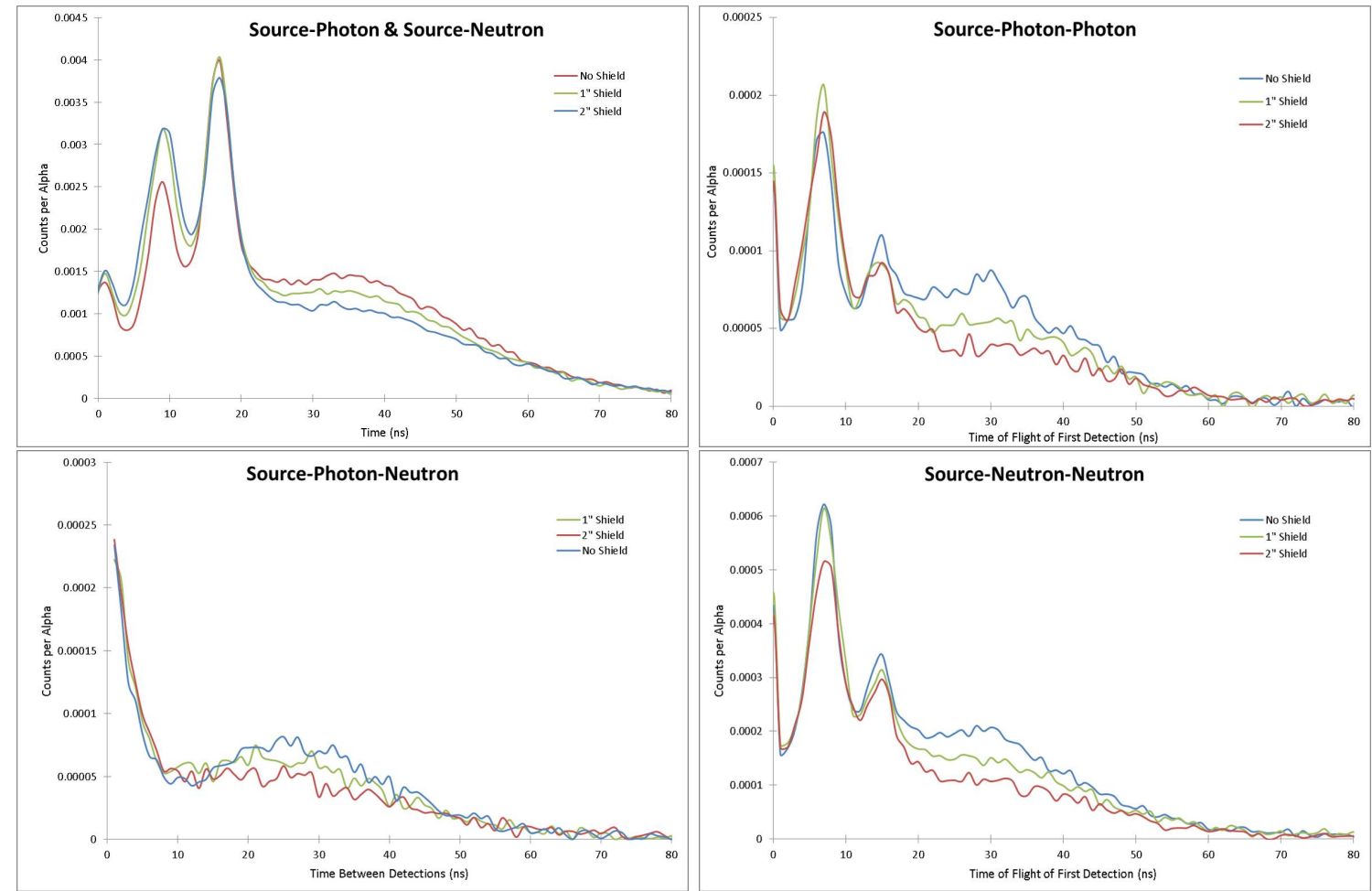

Figure 2.5: A study of DU signatures with two levels of aluminum shielding. 

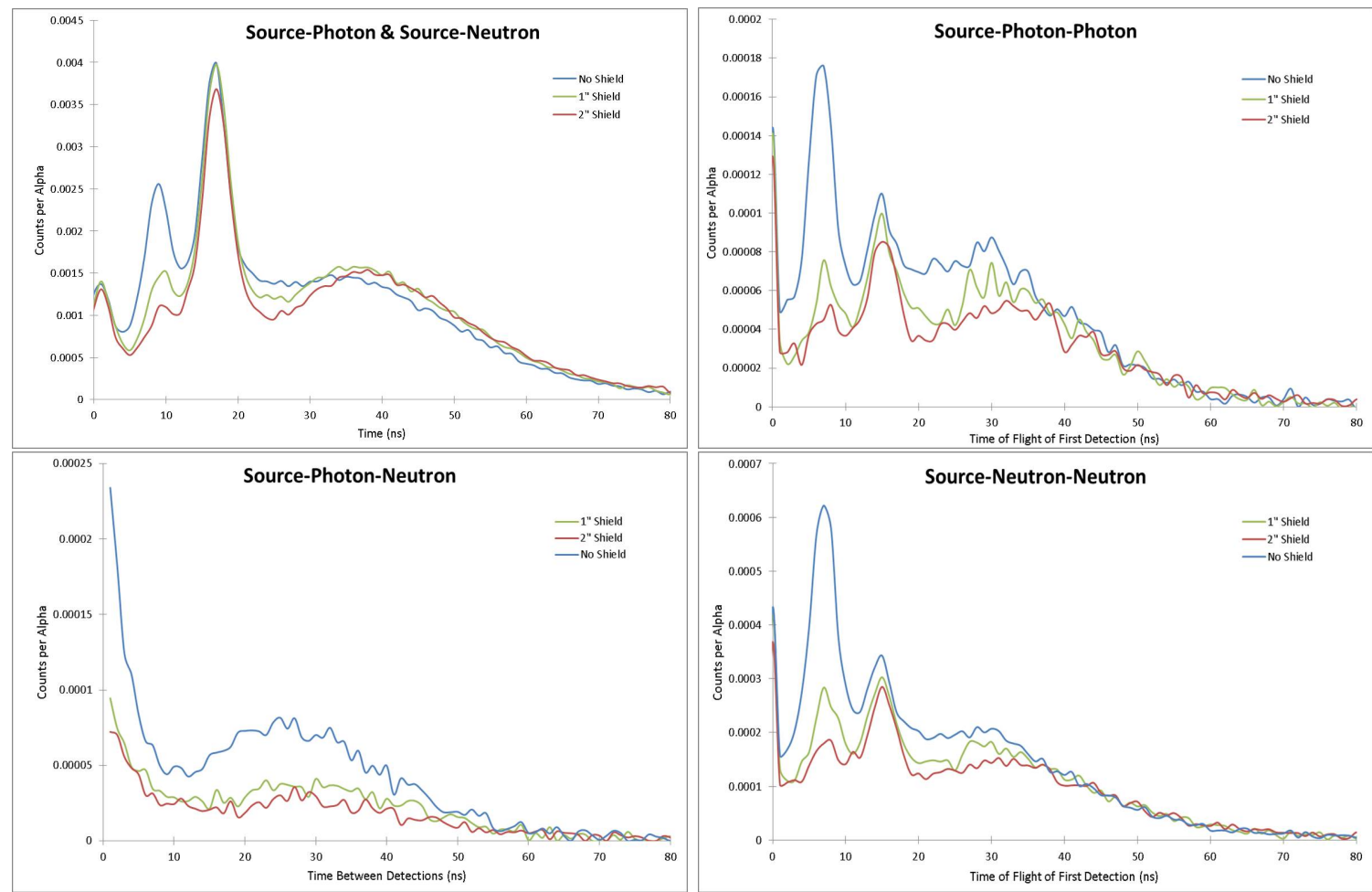

Figure 2.6: A study of DU signatures with two levels of lead shielding. 

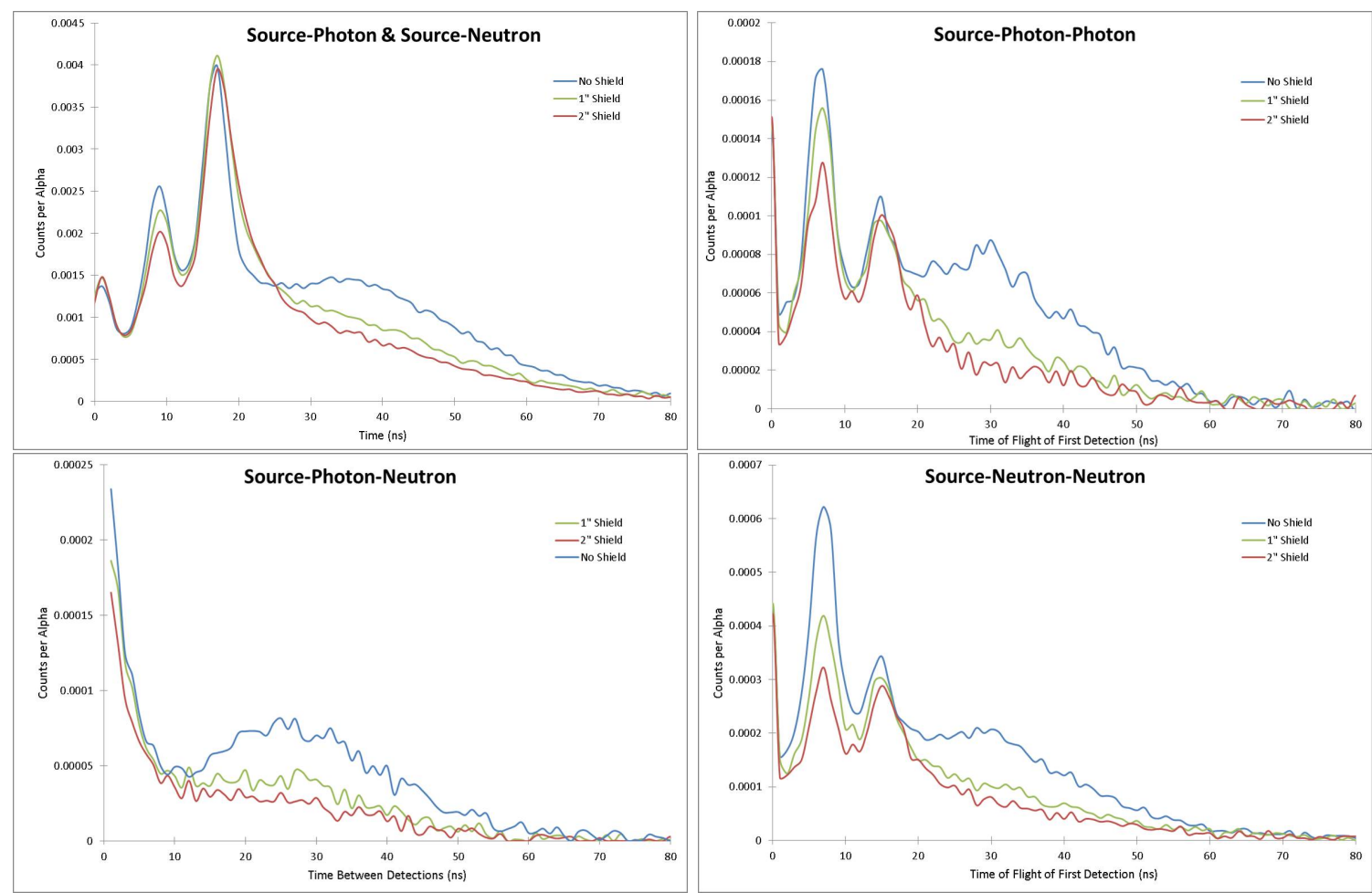

Figure 2.7: A study of DU signatures with two levels of polyethylene shielding. 


\subsection{MCNP Simulations of API Detection System}

MCNP-PoliMi/PolimiX (38) are a versions of MCNP/MCNPX that are used when researchers need physical accuracy on an event by event basis. In MCNP5 and MCNPx, energy is not conserved on an event by event basis, but the particle tallies average out to conserve energy. Since this research correlates detection with a source event and between detectors, event by event accuracy is required. PoliMi also added some source definitions, improved the induced fission multiplicity distribution, as well as some other adjustments.

The MCNP PoliMi input decks for NMIS simulations are generated by a code written by James Henkel at ORNL, which creates separate input decks for each alpha pixel viewpoint. All of the data presented here is from the field of view of pixel 8 (out of 16 pixels). The source neutron rate as seen by pixel 8 is modeled as a oval spot that tapers off in strength from the center in a Gaussian manner, which was determined by fitting to data. The view of the source looks almost directly through the center of the inspection object. Figure 2.8 shows the cross sectional field of view of pixel 8 by plotting the locations of the first interactions of the source particles, with the circles marking the inner and outer radius of the hollow cylindrical uranium casting and the outer radius of the $10 \mathrm{~cm}$ of shielding. The post processor was written by Brandon Grogan at ORNL(39) and updated by James Henkel and myself, and it parses the large "list mode" output file generated by MCNP PoliMi. The code attempts to replicate signals from a detector system by simulating detector dead time, calculating the light output of each pulse, sampling a Guassian distribution to realistically vary the energy deposited and timing information, and simulating detector thresholds. The code outputs data in various forms, including gamma-ray energy spectra and source correlated and bicorrelated time-of-flight plots. 


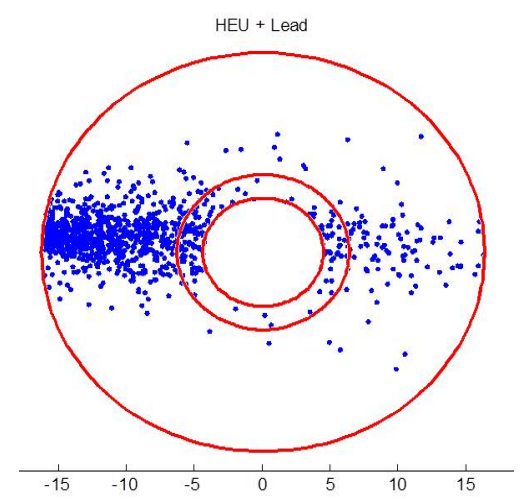

Figure 2.8: The first interaction of the source particles were plotted for the input deck of the alpha pixel 8 field of view.

\subsubsection{Comparison of Data to Simulations}

Comparing two data sets taken by the same equipment with the same calibration, such as the comparison done in the previous section, eliminates a lot of the uncertainty about the detector system parameters. Comparing data to simulations of a fairly complex detector system leads to a lot of places for potential small inaccuracies and uncertainties to add up to give significantly different results. As is described in Appendix B, care was taken to closely model the detector system, including a measurement series to investigate the position sensitivity of the detectors, which is described in Appendix A. Additionally, MCNP PoliMi, the 3-D radiation transport Monte Carlo simulation code being used, draws from some nuclear data and physics models that are not the most recent; even the most recent nuclear data is known to need improvement, especially for neutron and photon data from fission (40).

Given these challenges, the differences in the signatures, which can be seen in Figure 2.9, are not surprising. The source-photon signatures are significantly different, but part of this appears to be the way that timing uncertainty was modeled 


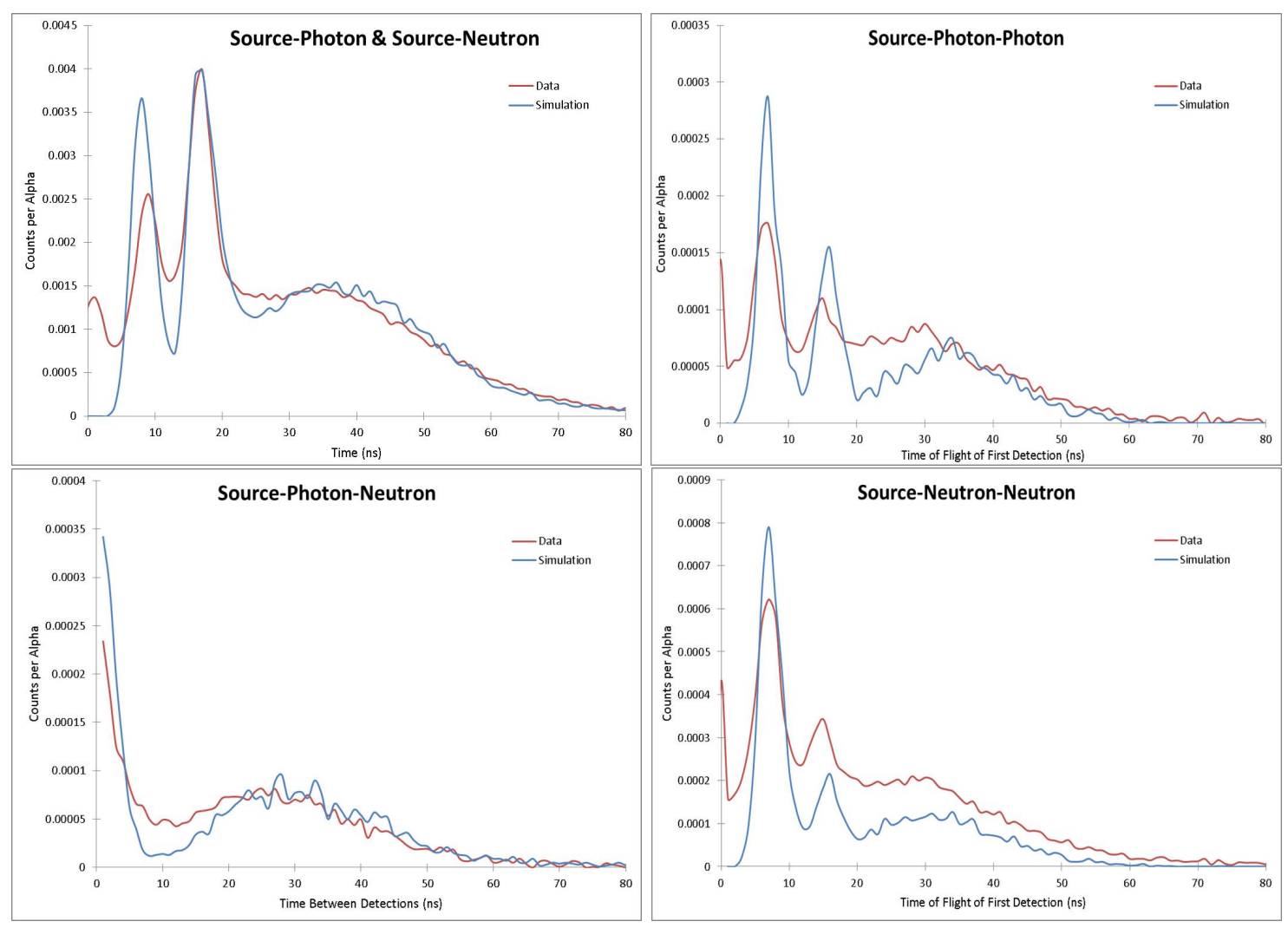

Figure 2.9: A comparison between data and simulation for all signatures.

in the post processor, since the peak shapes aren't quite the same. The sourceneutron signature is an impressively good match, though it appears that there are more scattered neutrons reaching the detectors in the data than the simulation. The source-photon-photon peaks have different shapes between data and simulation, but the integral looks to be similar. The source-photon-neutron signature is very similar, which is a very interesting result. The source-neutron-neutron peak is the worst fit, which is surprising given the close fit of the source-neutron peak. It appears that there may be some background that is not being modeled in source scattering and in the source-neutron-neutron time region, but further investigations would be needed to confirm this.

The comparison between simulation and data is shown in Table 2.3. The results 
are largely consistent with conclusions drawn from Figure 2.9; The source-neutron and source-photon-neutron signatures compare the best between data and simulation. They vary in a random way, sometimes being over predicted by simulation and other times being under predicted. Since the material is changing in every measurement, these small changing variations could be largely influenced by differences in nuclear data and uncertainties/inaccuracies in modeling each material type.

On the other hand, the source-neutron-neutron signature is under predicted by the simulations in a very similar manner across all measurements. The standard deviation of the "\% Difference" column for the source-neutron-neutron signature is only $22 \%$, which is very low considering that all measurements had different target objects. This points to a systematic difference between the experimental setup and the model, regardless of the inspection object.

The source-photon and source-photon-photon signature also appears to have some systematic differences, since the simulations routinely over predict the data. These vary more wildly in comparison, so the differences are likely due to a variety of culprits. The photon data from $14 \mathrm{MeV}$ induced fission is known to need improvement, which should be kept in mind (40). It is interesting to note how similar the differences are between all DU and tungsten configurations when comparing simulations and data, which implies that the fission interactions within DU are not the primary culprit for inaccuracy.

The most interesting results of this comparison is the closeness of fit between the source-photon-neutron simulations and data. Because it is a third order correlation and resistant to cross talk, this signature would be less sensitive to changing background rates than any of the other signatures. However, there's also a chance that this signature's good fit is too good to be true; The severe under prediction of the source-neutron-neutron signature and the significant over prediction of the 


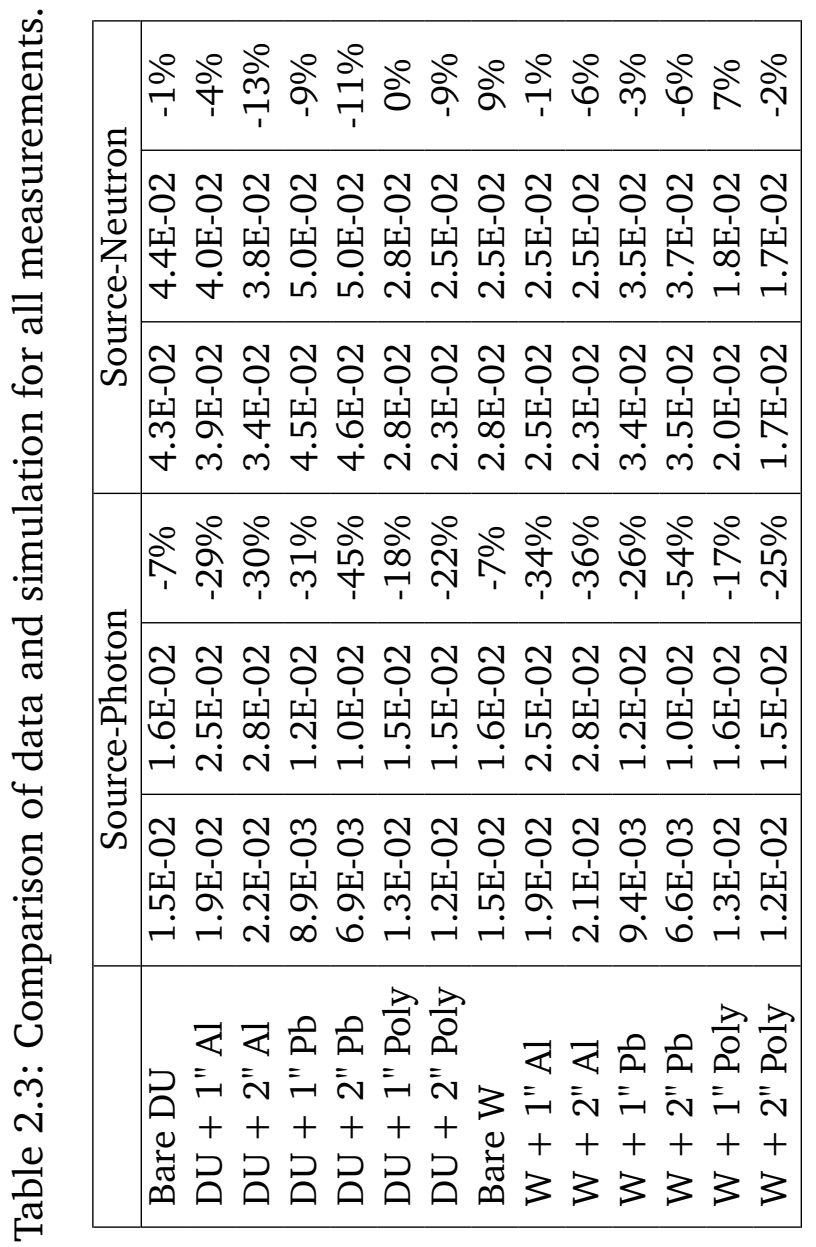

\begin{tabular}{|c|c|}
\hline & 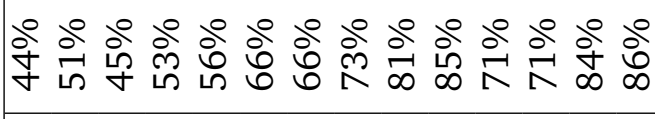 \\
\hline & 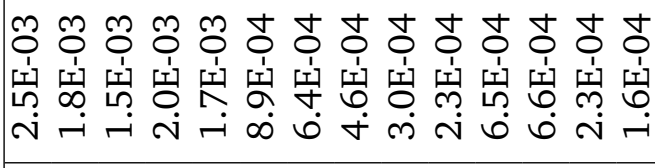 \\
\hline & 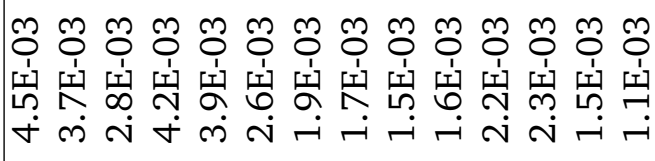 \\
\hline & 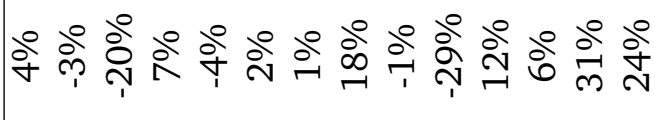 \\
\hline & 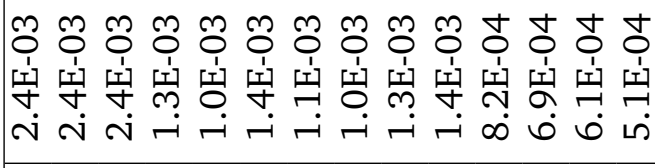 \\
\hline & 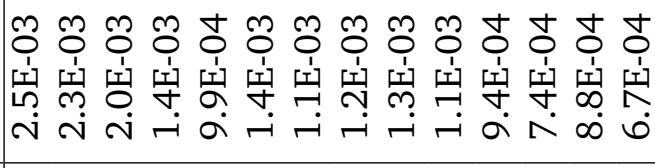 \\
\hline & 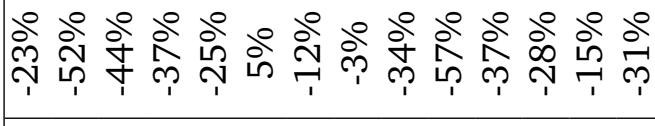 \\
\hline & 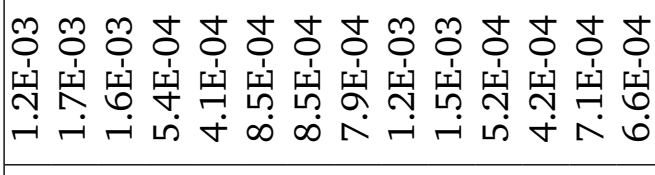 \\
\hline & 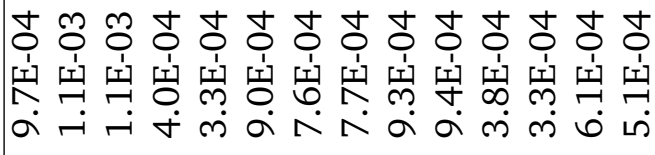 \\
\hline & $D_{0}$ \\
\hline
\end{tabular}


source-photon-photon signature could mean that the issues underlying these two signatures could be canceling out in the source-photon-neutron rate. Never the less, the results are promising and the signature is worth exploring further.

The closeness of fit of the source-neutron signature was also a promising result. This signature was already known to be a good indicator of enrichment (41), but matching the data and simulations for detectors as large as these was troublesome in the past (34).

\subsubsection{Varying Enrichment in Simulations}

Moving forward into purely simulation space, the differences between simulation and data from the previous section will be taken to be the confidence level for each of these signature types for each configuration. Additional simulations of all shielded and bare DU measurements were done with the enrichment gradually increased. These simulations of varied enrichment will be used to answer the following question:

$\rightarrow$ Given the ability of the simulations to predict the measured data in the previous section, to what extent can each of these signatures be used to predict uranium enrichment via simulation?

This section will explore the predictive ability of the simulations given both the sensitivity of the signatures to enrichment and the closeness of fit between data and simulation determined by the previous measurements.

Each uranium measurement was simulated with the following enrichment levels:

- $0.3 \%(\mathrm{DU})$ 
- $20 \%$

- $40 \%$

- $60 \%$

- $80 \%$

- $100 \%$

Figure 2.10 shows all signatures for various uranium enrichments. All signatures are clearly sensitive to enrichment, and so for this unshielded uranium configuration, all signatures are reasonable candidates for enrichment prediction.

Figure 2.11 shows all signatures for various enrichments of uranium shielded by 2" aluminum. The signatures are less well separated in this shielded configuration, as is expected. The source-photon signatures are quite close together due to the fission signature being washed out by the inelastic scattering gamma-ray contribution. All other signatures remain reasonably well separated.

Figure 2.12 shows all signatures for various enrichments of uranium shielded by 2 " of lead. Lead has a high (n, 2n) interaction rate, and also will affect the multiplication of the configuration by scattering some neutrons back into the uranium. Therefore, an increased neutron rate is actually seen with the lead shielding. The photon peak is barely distinguishable in the various enrichments, and would not be a good candidate for characterization. This result is not very surprising.

Figure 2.13 shows all signatures for various enrichments of uranium shielded by 2 " of polyethylene. Here the neutron signature is not as well separated, though still relatively distinguishable. The photon peaks are generally easy to distinguish, especially for source-photon-photon rates. 

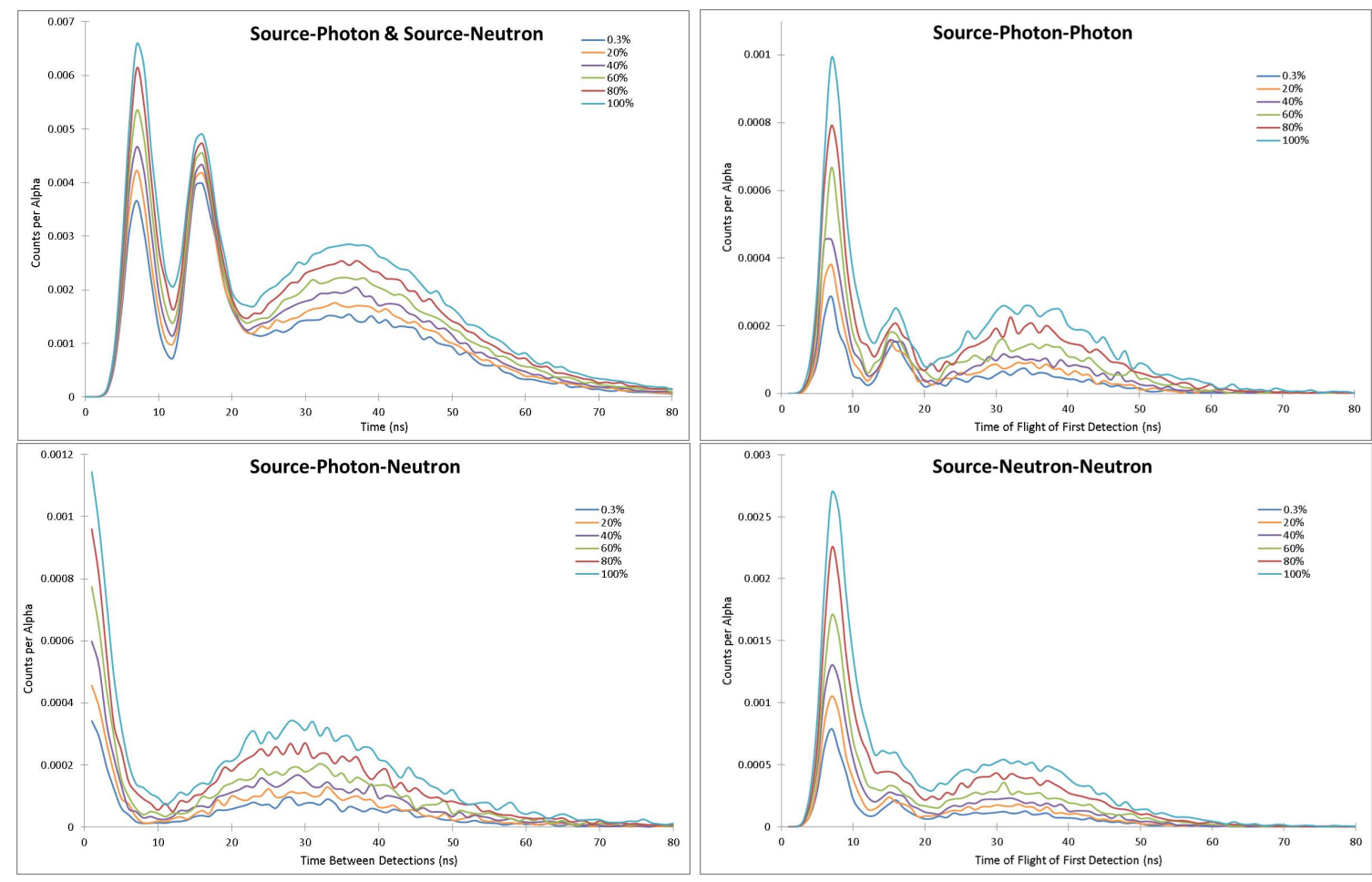

Figure 2.10: All signatures with variable enrichments of bare uranium.

Tables $2.4-2.7$ show the summary of all the signature rates for all enrichments for each of the four configurations.

\subsubsection{Predicting Enrichment with Simulations}

As a way to judge how effective these signatures are at informing about enrichment, a simple study was done. The percent difference from Section 2.5.1, given in Table 2.3, was taken to be the precision of the each signature. These uncertainties are repeated in Tables $2.4-2.7$ in the rows labeled "\% Diff." Each enrichment vs signature rate was plotted and a function was fit to the plot. Then the following questions were answered:

- If the sample was $100 \%{ }^{235} \mathrm{U}$, based on the precision level, what is the lowest possible enrichment that the signatures would predict? 

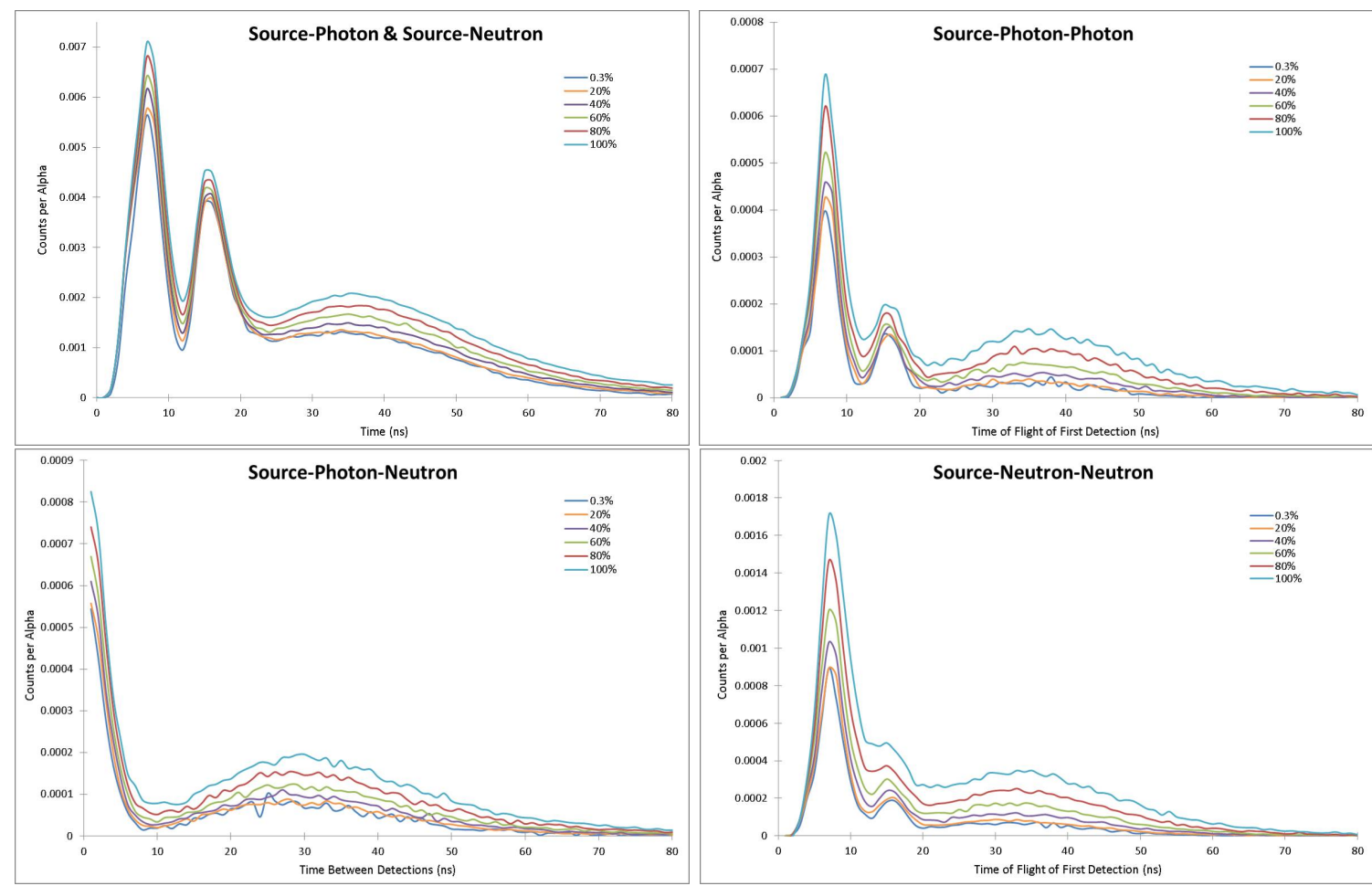

Figure 2.11: All signatures with variable enrichments of uranium shielded by 2 " of aluminum.

- If the sample was DU, based on the precision level, what is the highest possible enrichment that the signatures would predict?

Figure 2.14 shows an example of how these questions were answered; The enrichment vs source-photon-photon rate for bare uranium are plotted, and a third order polynomial is fit to the simulated data points. When comparing simulations to data in the previous section, it was determined that the $\%$ difference between the source-photon-photon rate for unshielded uranium was 23\%. Figure 2.14 shows how this level of confidence in signature rate translates into a confidence in enrichment determination, if the casting being measured were $100 \%{ }^{235} \mathrm{U}$. Because the photon rate is only known to within $\pm 23 \%$, the enrichment of a $100 \%$ enriched uranium casting could only be determined to be greater than $80 \%$ enriched, which 


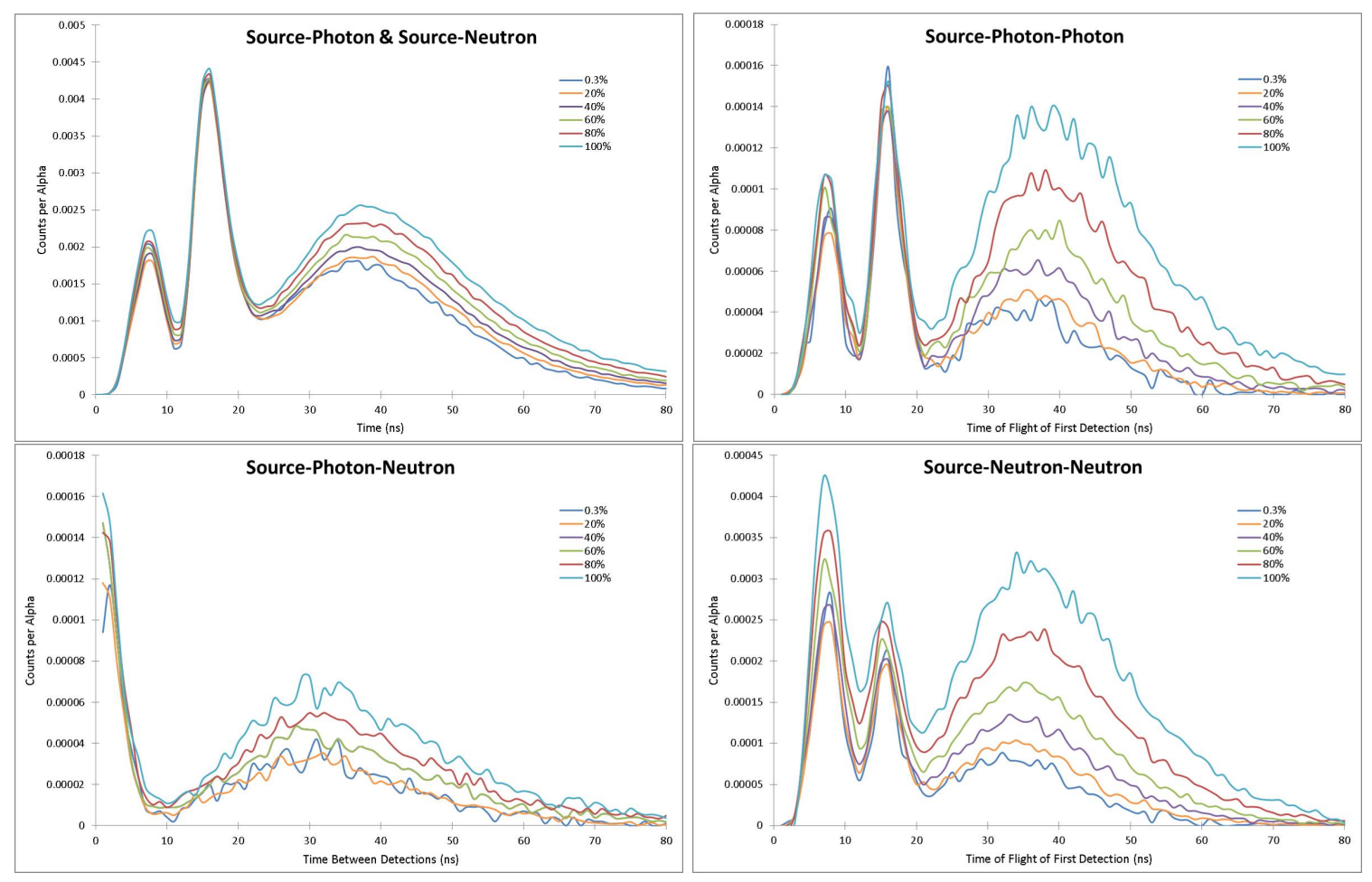

Figure 2.12: All signatures with variable enrichments of uranium shielded by 2 " of lead.

is reflected in Table 2.4 .

This helps to inform about the usefulness of these signatures for enrichment prediction, accounting for the variability of the signature with enrichment, and the ability of the simulation tool to predict this behavior. If a signature predicts that a $100 \%$ enriched casting is anywhere from 10\% - 100\% enriched, that is not very useful, because the sample could potentially be LEU or HEU. It should be noted that this is a simple study meant to derive meaning from the simulations and measurements taken in this project. A more rigorous study would involve a full uncertainty analysis that takes into account more than just counting statistics, as a way of understanding the main sources of uncertainty that are contributing the the differences between the simulations and measurements. 


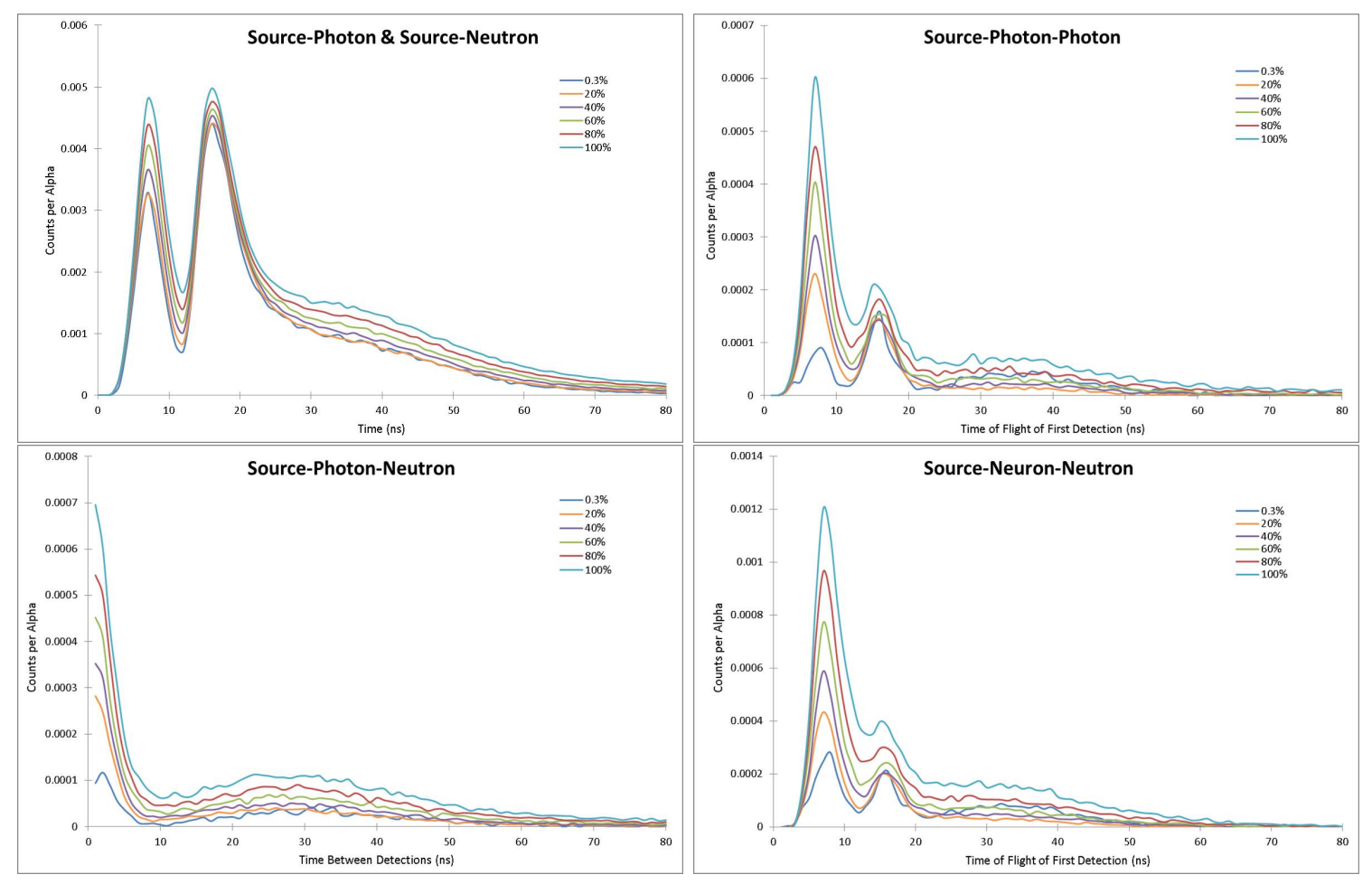

Figure 2.13: All signatures with variable enrichments of uranium shielded by 2 " of polyethylene.

The results of this simple study are given in Tables 2.4 - 2.7. In the bare configuration, all signatures were able to predict the enrichment fairly well. The sourceneutron-neutron signature suffered the greatest due to the low precision level determined in the Section 2.5.1.

When shielding is added, the strengths of certain signatures come into play. As would be expected, the source-photon signature was not useful in predicting the enrichment of an aluminum shielded configuration, and many of the other signatures were also not informative. All signatures could potentially mistake DU for HEU (defined as $>20 \%$ ). This is due to the significant discrepancies between simulation and experiment for all aluminum shielded configurations, and the high rate of inelastic scattering gamma-rays that interfere with the photon signature compared the relative light atoms which cause moderate amounts of thermalization of 


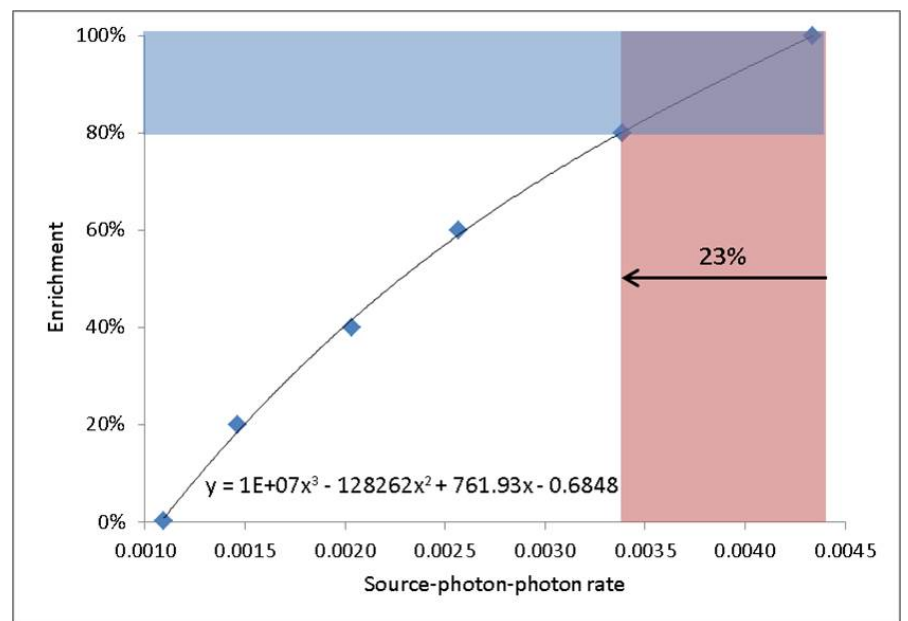

Figure 2.14: Example to illustrate the enrichment determinations in Tables 2.4 - 2.7

the neutron signature. The source-neutron-neutron signature was the most useful, despite the low precision level and moderate thermalization. This is not surprising since the source-neutron-neutron rate should be quite sensitive to enrichment due to the third order correlation and because neutrons are not highly affected by aluminum. It is interesting that the source-photon-neutron signature was somewhat useful as well.

For lead shielding uranium, it is unsurprising that the source-photon and sourcephoton-photon peaks are not useful. However, the source-neutron and sourceneutron-neutron signatures are both somewhat useful in predicting enrichment, which is also not surprising. It is again surprising that the source-photon-neutron signature was so useful in enrichment prediction ability, able to predict the $100 \%$ enriched sample as HEU, and the DU sample as LEU or below.

The polyethylene shielded uranium configurations gave interesting results. The source-neutron signature was a better predictor of enrichment than the sourcephoton signature, partially due to their respective precision levels. This result is counter-intuitive, but the bicorrelation signature results are aligned with expecta- 
Table 2.4: Signatures of bare uranium varying in enrichment

\begin{tabular}{|c|c|c|c|c|c|}
\hline Enrichment & S-P & $\mathrm{S}-\mathrm{N}$ & S-P-P & S-P-N & S-N-N \\
\hline $0.3 \%$ & $1.6 \mathrm{E}-02$ & 4.4E-02 & $1.1 \mathrm{E}-03$ & $2.4 \mathrm{E}-03$ & $1.1 \mathrm{E}-03$ \\
\hline $20 \%$ & $1.9 \mathrm{E}-02$ & 5.0E-02 & $1.5 \mathrm{E}-03$ & 3.3E-03 & $1.6 \mathrm{E}-03$ \\
\hline $40 \%$ & 2.2E-02 & 5.7E-02 & 2.0E-03 & 4.5E-03 & $2.2 \mathrm{E}-03$ \\
\hline $60 \%$ & $2.6 \mathrm{E}-02$ & $6.4 \mathrm{E}-02$ & 2.6E-03 & 5.7E-03 & $3.2 \mathrm{E}-03$ \\
\hline $80 \%$ & $2.8 \mathrm{E}-02$ & 7.4E-02 & $3.4 \mathrm{E}-03$ & 7.7E-03 & 4.6E-03 \\
\hline $100 \%$ & $3.1 \mathrm{E}-02$ & 8.4E-02 & 4.3E-03 & 1.0E-02 & 6.3E-03 \\
\hline \% Difference (from Table 2.3) & $\pm 7 \%$ & $\pm 1 \%$ & $\pm 23 \%$ & $\pm 4 \%$ & $\pm 44 \%$ \\
\hline $\begin{array}{l}\text { If sample was pure U235, } \\
\text { could predict that enrichment } \\
\text { was no less than . . . }\end{array}$ & 85 wt. $\%$ & 99 wt. $\%$ & 80 wt. $\%$ & 95 wt. $\%$ & 66 wt. $\%$ \\
\hline $\begin{array}{l}\text { If sample was DU, could } \\
\text { predict that the enrichment } \\
\text { was no greater than. . . }\end{array}$ & 9 wt. $\%$ & $1 \mathrm{wt} . \%$ & 13 wt. $\%$ & $1 \mathrm{wt} . \%$ & 21 wt. $\%$ \\
\hline
\end{tabular}

tions. The source-photon-photon signature is an effective enrichment predictor, while the source-neutron-neutron signature is less effective. Again the sourcephoton-neutron signature is an excellent signature for predicting enrichment, primarily due to its high precision level. 
Table 2.5: Signatures of uranium shielded by 2 " of aluminum varying in enrichment

\begin{tabular}{|l|ccccc|}
\hline \multicolumn{1}{|c|}{ Enrichment } & S-P & S-N & S-P-P & S-P-N & S-N-N \\
\hline $0.3 \%$ & $2.8 \mathrm{E}-02$ & $3.8 \mathrm{E}-02$ & $1.6 \mathrm{E}-03$ & $2.4 \mathrm{E}-03$ & $7.3 \mathrm{E}-04$ \\
$20 \%$ & $3.2 \mathrm{E}-02$ & $4.0 \mathrm{E}-02$ & $1.8 \mathrm{E}-03$ & $2.7 \mathrm{E}-03$ & $9.8 \mathrm{E}-04$ \\
$40 \%$ & $3.3 \mathrm{E}-02$ & $4.2 \mathrm{E}-02$ & $2.1 \mathrm{E}-03$ & $3.3 \mathrm{E}-03$ & $1.6 \mathrm{E}-03$ \\
$60 \%$ & $3.5 \mathrm{E}-02$ & $4.4 \mathrm{E}-02$ & $2.3 \mathrm{E}-03$ & $4.1 \mathrm{E}-03$ & $2.4 \mathrm{E}-03$ \\
$80 \%$ & $3.6 \mathrm{E}-02$ & $4.6 \mathrm{E}-02$ & $2.7 \mathrm{E}-03$ & $5.3 \mathrm{E}-03$ & $3.7 \mathrm{E}-03$ \\
$100 \%$ & $3.9 \mathrm{E}-02$ & $4.9 \mathrm{E}-02$ & $3.1 \mathrm{E}-03$ & $6.8 \mathrm{E}-03$ & $5.7 \mathrm{E}-03$ \\
\multicolumn{1}{|c|}{ $\pm 29 \%$} & $\pm 13 \%$ & $\pm 44 \%$ & $\pm 20 \%$ & $\pm 45 \%$ \\
\hline \% Difference (from Table 2.3) & \pm 20 & & & \\
\hline $\begin{array}{l}\text { If sample was pure }{ }^{235} \mathrm{U}, \\
\text { could predict that enrichment }\end{array}$ & $\mathrm{NA}$ & $46 \mathrm{wt} \%$ & $13 \mathrm{wt} \%$ & $82 \mathrm{wt} \%$ & $72 \mathrm{wt} . \%$ \\
was no less than . . & & & & & \\
\hline $\begin{array}{l}\text { If sample was DU, could } \\
\text { predict that the enrichment } \\
\text { was no greater than. . }\end{array}$ & $67 \mathrm{wt} \%$ & $50 \mathrm{wt} \%$ & $59 \mathrm{wt} \%$ & $40 \mathrm{wt} . \%$ & $21 \mathrm{wt} . \%$ \\
\hline
\end{tabular}

Table 2.6: Signatures of uranium shielded by 2" of lead varying in enrichment

\begin{tabular}{|l|ccccc|}
\hline \multicolumn{1}{|c|}{ Enrichment } & S-P & S-N & S-P-P & S-P-N & S-N-N \\
\hline \multicolumn{1}{|c}{$0.3 \%$} & $9.8 \mathrm{E}-03$ & $5.0 \mathrm{E}-02$ & $3.9 \mathrm{E}-04$ & $1.0 \mathrm{E}-03$ & $8.9 \mathrm{E}-04$ \\
$20 \%$ & $9.5 \mathrm{E}-03$ & $5.5 \mathrm{E}-02$ & $4.0 \mathrm{E}-04$ & $1.0 \mathrm{E}-03$ & $1.3 \mathrm{E}-03$ \\
$40 \%$ & $1.0 \mathrm{E}-02$ & $6.0 \mathrm{E}-02$ & $4.4 \mathrm{E}-04$ & $1.4 \mathrm{E}-03$ & $1.9 \mathrm{E}-03$ \\
$60 \%$ & $1.1 \mathrm{E}-02$ & $6.6 \mathrm{E}-02$ & $5.1 \mathrm{E}-04$ & $1.4 \mathrm{E}-03$ & $2.7 \mathrm{E}-03$ \\
$80 \%$ & $1.1 \mathrm{E}-02$ & $7.3 \mathrm{E}-02$ & $5.5 \mathrm{E}-04$ & $1.8 \mathrm{E}-03$ & $4.0 \mathrm{E}-03$ \\
$100 \%$ & $1.2 \mathrm{E}-02$ & $8.2 \mathrm{E}-02$ & $5.9 \mathrm{E}-04$ & $2.2 \mathrm{E}-03$ & $5.9 \mathrm{E}-03$ \\
\hline \% Difference (from Table 2.3) & $\pm 45 \%$ & $\pm 11 \%$ & $\pm 25 \%$ & $\pm 4 \%$ & $\pm 56 \%$ \\
\hline $\begin{array}{l}\text { If sample was pure }{ }^{235} \mathrm{U}, \\
\text { could predict that enrichment }\end{array}$ & $\mathrm{NA}$ & $79 \mathrm{wt} \%$ & $37 \mathrm{wt} . \%$ & $97 \mathrm{wt} \%$ & $58 \mathrm{wt} \%$ \\
was no less than . . & & & & & \\
\hline $\begin{array}{l}\text { If sample was DU, could } \\
\text { predict that the enrichment } \\
\text { was no greater than. . }\end{array}$ & $\mathrm{NA}$ & $23 \mathrm{wt} \%$ & $57 \mathrm{wt} . \%$ & $17 \mathrm{wt} \%$ & $24 \mathrm{wt} \%$ \\
\hline
\end{tabular}


Table 2.7: Signatures of uranium shielded by 2 " of polyethylene varying in enrichment

\begin{tabular}{|l|ccccc|}
\hline \multicolumn{1}{|c|}{ Enrichment } & S-P & S-N & S-P-P & S-P-N & S-N-N \\
\hline $0.3 \%$ & $1.5 \mathrm{E}-02$ & $2.5 \mathrm{E}-02$ & $8.5 \mathrm{E}-04$ & $1.1 \mathrm{E}-03$ & $2.6 \mathrm{E}-04$ \\
$20 \%$ & $1.6 \mathrm{E}-02$ & $2.7 \mathrm{E}-02$ & $9.6 \mathrm{E}-04$ & $1.2 \mathrm{E}-03$ & $3.1 \mathrm{E}-04$ \\
$40 \%$ & $1.8 \mathrm{E}-02$ & $3.1 \mathrm{E}-02$ & $1.3 \mathrm{E}-03$ & $1.7 \mathrm{E}-03$ & $5.5 \mathrm{E}-04$ \\
$60 \%$ & $2.0 \mathrm{E}-02$ & $3.4 \mathrm{E}-02$ & $1.7 \mathrm{E}-03$ & $2.3 \mathrm{E}-03$ & $8.4 \mathrm{E}-04$ \\
$80 \%$ & $2.3 \mathrm{E}-02$ & $4.0 \mathrm{E}-02$ & $2.1 \mathrm{E}-03$ & $3.1 \mathrm{E}-03$ & $1.5 \mathrm{E}-03$ \\
$100 \%$ & $2.5 \mathrm{E}-02$ & $4.6 \mathrm{E}-02$ & $2.7 \mathrm{E}-03$ & $4.3 \mathrm{E}-03$ & $2.3 \mathrm{E}-03$ \\
\hline \% Difference (from Table 2.3) & $\pm 22 \%$ & $\pm 9 \%$ & $\pm 12 \%$ & $\pm 1 \%$ & $\pm 66 \%$ \\
\hline $\begin{array}{l}\text { If sample was pure }{ }^{235} \mathrm{U}, \\
\text { could predict that enrichment }\end{array}$ & $55 \mathrm{wt} \% \%$ & $87 \mathrm{wt} \%$ & $89 \mathrm{wt} \%$ & $99 \mathrm{wt} \%$ & $54 \mathrm{wt} . \%$ \\
was no less than . . & & & & & \\
\hline $\begin{array}{l}\text { If sample was DU, could } \\
\text { predict that the enrichment }\end{array}$ & $38 \mathrm{wt} \%$ & $22 \mathrm{wt} \% \%$ & $14 \mathrm{wt} . \%$ & $1 \mathrm{wt} . \%$ & $28 \mathrm{wt} . \%$ \\
was no greater than. . & & & & \\
\hline
\end{tabular}

\subsection{Discussion}

Overall, all signatures investigated were sensitive to uranium enrichment. The usability of the signature is very dependent on the ability to model the signature accurately, though the accuracy of the model was improved with this work, and in many cases was good enough to differentiate HEU and DU. Modeling the signature accurately is also very dependent on how well the detector system components are characterized, such as the detector thresholds, source neutron cones, etc. The whole system is complex and it is not surprising that uncertainties from various sources add up to poor agreement, such as uncertainties in the source neutron shape, the detector cross talk, the induced background, the inspection object to source and detector distance, etc. A full uncertainty analysis of this system would be a considerable task, but would also greatly help in optimization of the system, since it is not currently clear what the largest sources of uncertainty are.

Based on both the simulations and experimental measurements, photon sig- 
natures were not a drastic improvement over neutron signatures in any shielding configurations. The source-photon signature has a variety of contributions besides fission gamma-rays, making it an unreliable signature for nuclear material characterization, as was evident by comparing the DU and tungsten source-photon rates. The source-photon-photon signature was a better measure of fissionable materials than neutron-only signatures for polyethylene shielding, but was also a low-rate signature. If source-neutron-neutron signatures could be modeled more accurately, it may be a better candidate for polyethylene since the source-neutron-neutron rate increases by nearly a factor of 10 over the span of enrichment, while the sourcephoton-photon rate increases by a factor of 3 .

The most interesting result was that the source-photon-neutron is a promising signature both in its close fit and sensitivity to enrichment in all configurations, able to differentiate low and high enriched uranium fairly well for all configuration, except aluminum, which appeared to be a problem modeling aluminum across all signatures. This was also an interesting result due to the signatures resistance to cross talk. 


\section{3 | Point Kinetics Analysis}

\subsection{Introduction}

In the previous chapter, conclusions were made that all the signatures were potentially useful for enrichment determination, though some signatures appeared to be more useful than others based on their resilience to shielding and their sensitivity to enrichment. An ideal API system and software would be able to take a high resolution image of an unknown object, which the software could use to input the object's geometry into a detailed 3-D radiation transport Monte Carlo code, and the simulation would be able to determine the enrichment by accurately and precisely matching the time-of-flight signatures discussed in Chapter 2 between measurement and simulation. However, the previous chapter illustrated the difficulty of using these detailed simulation tools to accurately model the complex associated particle active interrogation detector system; after much effort, the data and simulations still had significant differences, which led to unreliable enrichment calculations for certain shielded configurations.

On the other hand, this chapter investigates the utility of a simple point kinetics model to predict the same correlations discussed previously; in other words, this chapter uses the same signatures but a different prediction tool to relate the signatures to enrichment. The point kinetics model presented here uses a generating function technique to determine the number distribution of particles released from a subcritical fission chain. The basic structure of this point kinetics formulation 
has been applied to correlated detection systems for many years, which will be reviewed in the next section. This chapter will investigate how well this technique can be reformulated to apply to an API-DT generator based system.

While this chapter generally uses the same signatures that were explored in the previous chapter, the building of this point kinetics model allows for these signatures to be explored in more depth. The algorithms used in the previous chapter pulled out the first moments (source-photon, source-neutron) and second moments (source-photon-photon, source-photon-neutron, source-neutron-neutron) of the measured data. Any higher order coincidences that were measured are accounted for within those moments, but not directly realizable. For example, if a source neutron induces a fission which results in the detection of two photons and two neutrons, this one event would register as two source-photon and two-source-neutron correlation events, as well as one source-photon-photon, two source-photon-neutron, and one source-neutron-neutron bicorrelation events. However, the fact that a group of four particles were detected in coincidence would not be part of the current analysis. This chapter will expand out this dimension of these signatures, looking at how the level of multiplication leads to varying distributions of coincidences, which are collapsed into the moments that are used to analyze the inspection object.

It is important to understand throughout the discussion of this model that the purpose is not to have an exact formulation of the multiplying system, but to have a simple formulation that gives a close estimation. The underlying assumption in this model is based on point kinetics, which treats the system as a point with no mass or volume (though later in this discussion these assumptions will be undermined by attempting to correct for self-attenuation). The goal is to create a model that can use some simple nuclear data and give a close approximation of the system's mul- 
tiplicative characteristics. The inputs into the model are the number distributions of the neutrons and photons released during fission, as well as some simple group cross section data. It is also assumed that the geometry of the system is known, as in the case of a detection system capable of imaging an unknown object.

The goal of developing this point kinetics model is to tie the number of neutrons and photons released from a configuration to the multiplication of that configuration. Then the model can be used to approximate the multiplication based on detection rates of neutron and photon number distributions. The multiplication of a fissile uranium metal configuration is useful to know because it is a function of the total mass of uranium, the enrichment, the shielding material, and the geometry. Therefore if the mass, shielding, and geometry are known (via imaging) and the multiplication is determined via this point kinetics model, the enrichment can also be determined.

\subsubsection{Relevant Background}

The basic function for modeling the probabilities of detecting a given multiplet (number of particles emitted) by utilizing probability generating functions was formulated by Böhnel (42) in 1984, and extended to include detection efficiency, correlation gating methods, and time window corrections by Cifarelli and Hage (43; 44). Back then, the instruments used were typically well counters, which are composed of ${ }^{3} \mathrm{He}$ tubes embedded in a neutron moderating material. The inspection object would fission, either passively or induced by an AmBe source, and the fission neutrons would thermalize in the moderating material and be captured in the ${ }^{3} \mathrm{He}$ tubes for detection. The signatures were typically analyzed by their die-away characteristics, which was the detection of neutrons over time as they thermalized and 
were detected on the order of microseconds. The multiplication of the system was determined using one of several approaches based on their "singles," "doubles," and sometimes even "triples" coincidence rates (3).

The increased use of fast detectors, fast electronics, and the increased computing capabilities of recent years has caused fundamental shifts in coincidence detection, which has led to more research into extending correlation detection algorithms. The ability of fast detector systems to separately detect individual fission chains has led to many relooking at multiplicity counting algorithms. Thermal multiplicity counters measure chains over tens of microseconds, spreading the length of the chain out in time via thermalization and remaining blind to the photons. Using fast detector arrays shortens the time window to tens of nanoseconds and includes photon detection. This allows for detection of individual fission chains with reduced background because of the short time window, but also increases the importance of correctly accounting for dead time and ensuring correct particle classification, as well as other considerations.

The basic generating function point kinetics formulation was extended to include photon distributions within the last decade $(45 ; 46 ; 47)$. Chernikova et al. (48), primarily at Chalmers University of Technology, also continues to extend the branching theory laid out in Neutron Fluctuations in a Multiplying Medium Randomly Varying in Time (46). Their focus has been on including correlations of photons and neutrons from fission chains by looking at the covariance between photons and neutrons. The most recent work begins to include more interactions besides fission that affect number of neutrons and photons escaping an multiplying system, such as $(n, 2 n)$ interactions $(48)$.

Kim et al. (49) at Lawrence Livermore National Laboratory has extended the Feynman model of time evolving fission chains to connect them directly to the point 
model theory. They are able to model how a fission chain evolves in time based on the multiplication. Since the superior timing resolution of fast detection systems allow for detection of individual fission chains, this framework is directly connected to understanding fast detection of fission chains.

A research effort at Los Alamos National Laboratory and Oak Ridge National Laboratory has been looking at addressing some problems with the fundamentals of correlation counting analysis, and are working to improve both algorithms and signal processing techniques. Some of their focus areas include modeling dead time in multiplicity counters, addressing the spatial variation of multiplication within a multiplying object, multiple energy groups, and the potential utility of high order moments. Although their work is generally centered around thermal neutron counters, they have been going back to first principles to improve how these issues are modeled, which is applicable to both thermal and fast detection systems. (50)

Applying this basic generating function branching ratio technique to an API system is advantageous because of the minimal background and the precise knowledge of the chain initiation. One difficulty in multiplicity detection is modeling the gating schemes, given that the beginning of the chain is not known for passive systems. Another challenge is modeling the various types of background contributions, such as $(\alpha, \mathrm{n})$ interactions and ambient neutron backgrounds. Some work has been done previously on applying this point kinetics formulation to an active interrogation system (51), and will be extended here.

This chapter will begin by introducing the basic point kinetics framework in a similar manner to McConchie(51), which describes a system in which neutrons may escape the system or continue a fission chain. This basic Böhnel model will be extended to describe chains initiated by a $14 \mathrm{MeV}$ source neutron; next, a dual neutron and photon system will be presented; then the framework will be extended to in- 
clude more complex interactions; and finally detector efficiency will be considered. The interactions considered are fission interactions, induced by either fission energy neutrons or $14 \mathrm{MeV}$ neutrons; fission chains initiated by $14 \mathrm{MeV}(\mathrm{n}, 2 \mathrm{n})$ interactions instead of fission interactions; photon production via fission; photon absorption; and photon inelastic scattering. These interactions are considered because they were determined to have the most significant effects on the number distribution for small unmoderated uranium metal interrogated by $14 \mathrm{MeV}$ neutrons.

The framework will be developed by comparing to simulations of ideal systems. After the discussion of how this framework compares to ideal systems, the tone will change to more realistic systems. A simplified perturbation study will be done to investigate the sensitivities of various inputs to the multiplet uncertainties. Finally, some of the data from the previous chapter will be compared to the point kinetics model prediction to draw some conclusions about the usability of this technique and areas for improvement.

\subsection{The Böhnel Equation}

The probability generating function is a mathematical tool used to describe the number of occurrences of a given event that can happen. For example, the number of ways that 10 marbles could be distributed into 3 distinguishable jars is given by the coefficient in front of $y^{10}$ in the function

$$
f(y)=\left(1+y+y^{2}+\ldots y^{10}\right)^{3} .
$$


This coefficient could be determined with the following function

$$
\text { Coef }=\left.\frac{\left(\frac{d^{10}}{d y^{10}} f(y)\right)}{10 !}\right|_{y=0} .
$$

Böhnel (42) utilized this method to describe the probability of producing $n$ neutrons in a fission chain. Let $P_{n}(p)$ be the probability that $n$ neutrons do not produce further neutrons, but escape the system and are available for detection. This is a function of $p$ which is the probability that any given neutron will induce further fissions. The probability generating function, called $h(y)$, is given by

$$
h(y) \equiv \sum_{n=0}^{\infty} P_{n} y^{n}
$$

where the probability function $P_{n}(p)$ can be solved for by taking the $n$th partial derivative and evaluating at $y=0$

$$
P_{n}(p)=\left.\frac{1}{n !} \frac{d^{n}}{d y^{n}}(h(y))\right|_{y=0}=\left.\frac{h^{(n)}(y)}{n !}\right|_{y=0} .
$$

The function describing the initiation of a fission chain by a single neutron is

$$
h_{1}(y)=(1-p) y+p C(y) .
$$

The first term in this equation describes the probability that the initial neutron does not initiate a fission chain, and is available for detection. The second term incorporates the neutron multiplicity data from a fission interaction:

$$
C(y) \equiv \sum_{\nu=0}^{N_{c}} \alpha_{\nu}^{C} y^{\nu}
$$


where $\alpha_{\nu}^{C}$ is the probability of $\nu$ neutrons being released from the fission event and $N_{c}$ is the maximum number of neutrons that could be released from the fission event. The sum of the multiplet probabilities, $\alpha_{n u}^{C}$, are normalized. The neutrons released from this initial neutron can be plugged into the same equation to find the second generation of neutrons:

$$
h_{2}(y)=(1-p) y+p C\left(h_{1}(y)\right) .
$$

Again the first term describes a neutron that does not produce a fission reaction, and the second term describes the fission neutrons from the first generation going on to produce other fission interactions. The higher generations of the fission chain are described by continuing to plug previous generations into this generating function, which leads to the Böhnel equation (42):

$$
h(y)=(1-p) y+p C(h(y)) .
$$

The probability $P_{n}(p)$ that $n$ neutrons are released from a fission chain initiated by one neutron can then be determined by applying the $n$th derivative and solving for $y=0$

$$
P_{n}(p)=\left.\frac{h^{(n)}(y)}{n !}\right|_{y=0}=\left.\frac{(1-p) \delta_{n, 1}+p C^{(n)}(h(y))}{n !}\right|_{y=0}
$$

where the $n^{\text {th }}$ derivative of $C(h(y))$ can be expressed using Bruno's formula

$$
C^{(n)}(h(y))=\sum_{\theta\left(n ; N_{c}\right)} \frac{n !}{m_{1} ! m_{2} ! \ldots m_{n} !} C^{\left(m_{1}+m_{2}+\ldots m_{n}\right)}(h(y)) \prod_{j=1}^{n}\left(\frac{h^{(j)}}{j !}(y)\right)^{m_{j}}
$$


where the constraint $\theta\left(n, N_{c}\right)$ is defined as

$$
\theta\left(n, N_{c}\right): \sum_{j=1}^{n} j m_{j}=n \text { and } \sum_{j=n}^{n} m_{j} \leq N_{c}
$$

Putting this all together and inserting $h(0)=P_{0}(p)$, which just comes from the definition of $h(y), P_{n}(p)$ is found to be

$$
P_{n}(p)=(1-p) \delta_{n, 1}+p \sum_{\theta\left(n ; N_{c}\right)} \frac{1}{m_{1} ! m_{2} ! \ldots m_{n} !} C^{\left(m_{1}+m_{2}+\ldots m_{n}\right)}\left(P_{0}(p)\right) \prod_{j=1}^{n} P_{j}(p)^{m_{j}}
$$

This is equivalent to

$$
P_{n}(p)=\frac{(1-p) \delta_{n, 1}+p \sum_{\theta\left(n ; N_{c}\right)} \frac{1}{m_{1} ! m_{2} ! \ldots m_{n-1} !} C^{\left(m_{1}+m_{2}+\ldots m_{n-1}\right)}\left(P_{0}(p)\right) \prod_{j=1}^{n-1} P_{j}(p)^{m_{j}}}{\left(1-p C^{(1)}\left(P_{0}(p)\right)\right)}
$$

In this form, the value of $P_{n}(p)$ depends on the previous probability functions. This property allows the values to be found iteratively such that $P_{0}(p)$ can be used to determine $P_{1}(p)$ which then can be used to determine $P_{2}(p)$ and so on. It is useful to determine the moments of the probability generating function $h(y)$ because they end up being simpler. The moments of $h(y)$ are defined as

$$
\left.\Phi_{n}(p) \equiv \frac{h^{(n)}(y)}{n !}\right|_{y=1}=\sum_{\nu=n}^{\infty}\left(\begin{array}{l}
\nu \\
n
\end{array}\right) P_{\nu}(p)
$$

and given that $h(1)=\sum_{n=0}^{\infty} P_{n}(p)=1$, the following is applicable

$$
C^{(n)}(1)=\sum_{\nu=n}^{N_{c}} \frac{\nu !}{(\nu-n) !} \alpha_{\nu}^{C}=n ! \sum_{\nu=n}^{N_{c}}\left(\begin{array}{l}
\nu \\
n
\end{array}\right) \alpha_{\nu}^{C}=n ! \nu_{n}^{C}
$$


where $\nu_{n}^{C}$ is the $n^{\text {th }}$ moment of the number of neutrons emitted from a single fission. The $n^{\text {th }}$ moment is therefore

$$
\Phi_{n}(p)=\frac{(1-p) \delta_{n, 1}+p \sum_{\theta\left(n ; N_{c}\right)} \frac{\left(m_{1}+m_{2}+\ldots m_{n-1}\right) !}{m_{1} ! m_{2} ! \ldots m_{n-1} !} \nu_{\left(m_{1}+m_{2}+\ldots m_{n-1}\right)}^{C} \prod_{j=1}^{n-1} \Phi_{j}(p)^{m_{j}}}{\left(1-p \nu_{1}^{C}\right)}
$$

and the first few moments are given below:

$$
\begin{aligned}
\Phi_{1} & =\frac{1-p}{1-p \nu_{1}^{C}} \\
\Phi_{2} & =\left(\frac{1-p}{1-p \nu_{1}^{C}}\right)^{2}\left(\frac{p}{1-p \nu_{1}^{C}}\right) \nu_{2}^{C} \\
\Phi_{3} & =\left(\frac{1-p}{1-p \nu_{1}^{C}}\right)^{3}\left(\frac{p}{1-p \nu_{1}^{C}}\right)\left(\frac{2 p\left(\nu_{2}^{C}\right)^{2}}{1-p \nu_{1}^{C}}+\nu_{3}^{C}\right) .
\end{aligned}
$$

\subsection{Fission Chain Initiation}

The previous probability generating function described neutrons from induced fission propagating further fissions. However, the fission chain begins with one fission energy neutron, which is not applicable to the API system being modeled. For this work, the first fission of the chain will be induced by a $14 \mathrm{MeV}$ neutron. For now we'll assume that this $14 \mathrm{MeV}$ neutron interaction is a fission event, and later we'll consider $14 \mathrm{MeV}$ (n,2n) interactions as well. This initial $14 \mathrm{MeV}$ induced fission will have a different distribution and needs to be treated separately. First a probability generating function $H(y)$ is defined

$$
H(y) \equiv \sum_{n=0}^{\infty} P_{n}^{s}(p) y^{n} .
$$


such that $P_{n}^{s}(p)$ is the probability that $n$ neutrons are released from a fission chain that was started by a $14 \mathrm{MeV}$ induced fission. To account for this separate nuclear data, a function similar to $C(y)$ is defined, called $D(y)$, that consists of nuclear data of a $14 \mathrm{MeV}$ induced fission:

$$
D(y) \equiv \sum_{\nu=0}^{N_{c}} \alpha_{\nu}^{D} y^{\nu} .
$$

The Böhnel equation describes the chain propagated in the system per fission neutron, so a chain initiated by a $14 \mathrm{MeV}$ induced fission just needs to provide the probability of some number of neutrons, released by the initial fission, starting the chain described by Equation 3.8:

$$
\text { induced fission chain }=D(h(y))
$$

Additionally, the probability that the source $14 \mathrm{MeV}$ neutron induces a fission has to be accounted for. The incorporation of the $14 \mathrm{MeV}$ induced fission is similar to the previous analytic development. The source neutron will either not induce a fission, meaning that one neutron is released from the system, or the source neutron will initiate a chain described by the function $D(h(y))$ :

$$
H(y)=\left(1-\omega_{f}\right) y+\omega_{f} D(h(y)) .
$$

This probability is now a function of both $p$, the probability of any neutron inducing further fissions, and the coupling constant $\omega_{f}$, which is the probability that the source neutron will induce a fission. Equation 3.19 can then be solved for $P_{n}^{s}(p)$ in 
a similar manner as before:

$$
P_{n}^{s}(p)=\left(1-\omega_{f}\right) \delta_{n, 1}+\omega_{f} \sum_{\theta\left(n ; N_{D}\right)} \frac{1}{m_{1} ! m_{2} ! \ldots m_{n} !} D^{\left(m_{1}+m_{2}+\ldots m_{n}\right)}\left(P_{0}(p)\right) \prod_{j=1}^{n} P_{j}(p)^{m_{j}}
$$

Again, it is useful to find the moments of $P_{n}^{s}(p)$, given by

$$
\Psi_{n}(p)=\left(1-\omega_{f}\right) \delta_{n, 1}+\omega_{f} \sum_{\theta\left(n ; N_{D}\right)} \frac{\left(m_{1}+m_{2}+\ldots m_{n}\right) !}{m_{1} ! m_{2} ! \ldots m_{n} !} \nu_{\left(m_{1}+m_{2}+\ldots m_{n}\right)}^{D}\left(P_{0}(p)\right) \prod_{j=1}^{n} \Phi_{j}(p)^{m_{j}}
$$

The first few moments are

$$
\begin{aligned}
& \Psi_{1}=1-\omega_{f}+\omega_{f} \frac{1-p}{1-p \nu_{1}^{C}} \nu_{1}^{D} \\
& \Psi_{2}=\omega_{f}\left(\frac{1-p}{1-p \nu_{1}^{C}}\right)^{2}\left(\nu_{2}^{D}+\left(\frac{p}{1-p \nu_{1}^{C}}\right) \nu_{2}^{C} \nu_{1}^{D}\right) \\
& \Psi_{3}=\omega_{f}\left(\frac{1-p}{1-p \nu_{1}^{C}}\right)^{3}\left(\nu_{3}^{D}+\left(\frac{p}{1-p \nu_{1}^{C}}\right)\left(2 \nu_{2}^{C} \nu_{2}^{D}+\nu_{3}^{C} \nu_{1}^{D}\right)+\left(\frac{p}{1-p \nu_{1}^{C}}\right)^{2}+2\left(\nu_{2}^{C}\right)^{2} \nu_{1}^{D}\right) .
\end{aligned}
$$

As a way to build confidence in the point kinetics model described in the previous sections, the moments and probabilities are compared between the analytical equations and MCNPx PoliMi. The multiplet probabilities, described by the analytical function, were not solved for directly, but were determined by sampling the analytical equations in a Monte Carlo code*. These results were compared to MCNPx PoliMi models, in which the particles escaping the spheres were counted with a SSW tally. The SSW tally was post-processed to determine the multiplet probability by a Python code ${ }^{\dagger}$.

The objects used for comparison were various sizes of metal spheres of ${ }^{235} U$. For simplicity, the $14 \mathrm{MeV}$ source is distributed throughout the sphere evenly. Section

*This code was created initially by Seth McConchie and edited for this investigation

${ }^{\dagger}$ This code was written by Matthew Tweardy (52) and edited for use in this work 
3.6.7 will describe the calculations of the input values. Appendix $\mathrm{C}$ describes how to calculate the average distance traveled within each sphere, which is needed to determine the $\omega_{f}$ value for each sphere.

Figure 3.1 shows the distributions as described by Equation 3.19 compared to neutrons escaping $1 \mathrm{~cm}, 4 \mathrm{~cm}$, and $6 \mathrm{~cm}$ spheres of 100\% enriched uranium, as modeled by MCNPx PoliMi. For each case, the multiplet probability and the first two moments are given for comparison. The fit between point kinetics and MCNP is impressive considering that only fission events are being modeled. It makes sense that the fit is the worst around multiplets of 2 and that the MCNP moments are routinely higher than point kinetics (\% Diff is determined by $\frac{M C N P-P K}{M C N P}$ ). The (n,2n) interactions are significant in uranium for high energy neutrons and will be incorporated into the equations in Section 3.6.2. 


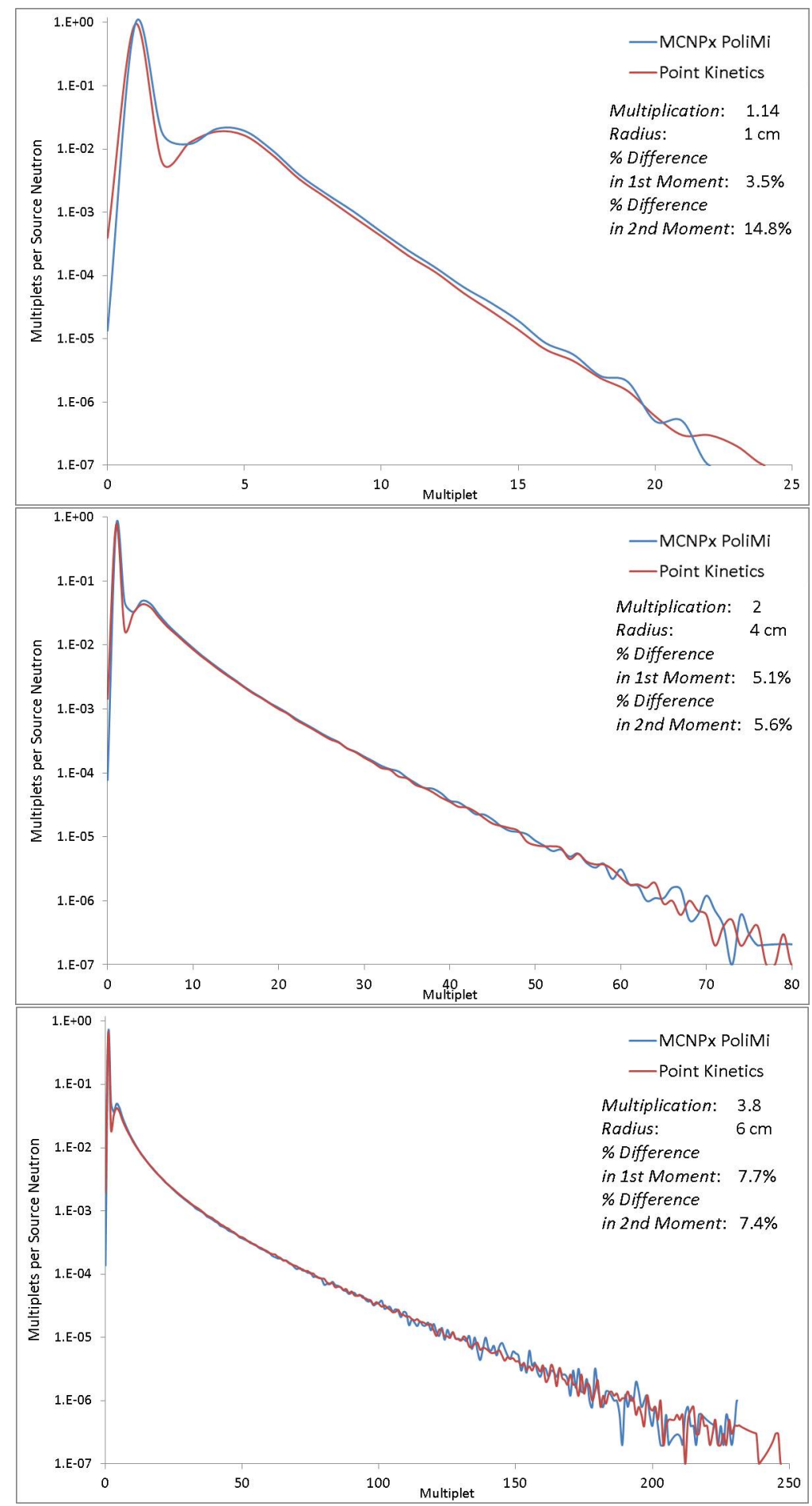

Figure 3.1: Comparison of MCNPx PoliMi output to point kinetics model (Equation 3.19) of neutrons escaping from ${ }^{235} U$ metal spheres of 1,4 and $6 \mathrm{~cm}$ in radius respectively. 


\subsection{Photon Distribution}

Adding photon distributions to this modeling technique has already been explored, and is presented in more depth in (46). The formulation described by them will briefly be presented here.

Since photons produced from fission interactions do not have enough energy to induce fission, the propagating mechanism for the fission chains is still driven by neutrons. This means that the photon distribution relies heavily on the neutron distribution. A function $E(x)$ is used to describe the distribution of photons released per fission interaction.

$$
E(x) \equiv \sum_{\nu=0}^{N_{c}} \alpha_{\nu}^{E} x^{\nu}
$$

To differentiate photons from neutrons, the variable $x$ is used instead of $y$ and the probability generating function is defined by

$$
g(x) \equiv \sum_{n=0}^{\infty} P_{n}^{\gamma} x^{n}
$$

and the equivalent expression for photons to the Böhnel equation is

$$
g(x)=(1-p)+p E(x) C(g(x))
$$

where the first term shows that no photons are emitted if no fission occurs, $(1-p)$, but are emitted in the event of a fission, $p E(x) C(g(x))$.

Just as in the neutron case, the distribution of photons emitted from initiation of a $14 \mathrm{MeV}$ fission chain needs to be treated separately than the fission neutron induced chains. Defining $F(x)$ to be the function describing the photon distribution from the $14 \mathrm{MeV}$ induced fission, the photon equivalent generating function and 
distribution function to Equation 3.19 is given by

$$
G(x) \equiv \sum_{n=0}^{\infty} P_{n}^{s, \gamma} x^{n}
$$

and

$$
G(x)=\left(1-\omega_{f}\right)+\omega_{f} F(x) D(g(x))
$$

Figure 3.2 shows a comparison of MCNP and point kinetics (Equation 3.25. This tally was done differently from that of Figure 3.1, because the photon distribution is heavily affected by other interactions besides fission, unlike the neutron case. Instead of comparing the particles escaping from the spheres, all fission photons produced in the spheres were tallied. This was done as a "sanity check" in order to show how well Equation 3.25 models the photons produced. It is clear that Equation 3.25 is a good representation of photons produced. The flux of photons escaping the spheres looks very different due primarily to self absorption, which will be accounted for in an upcoming section. 


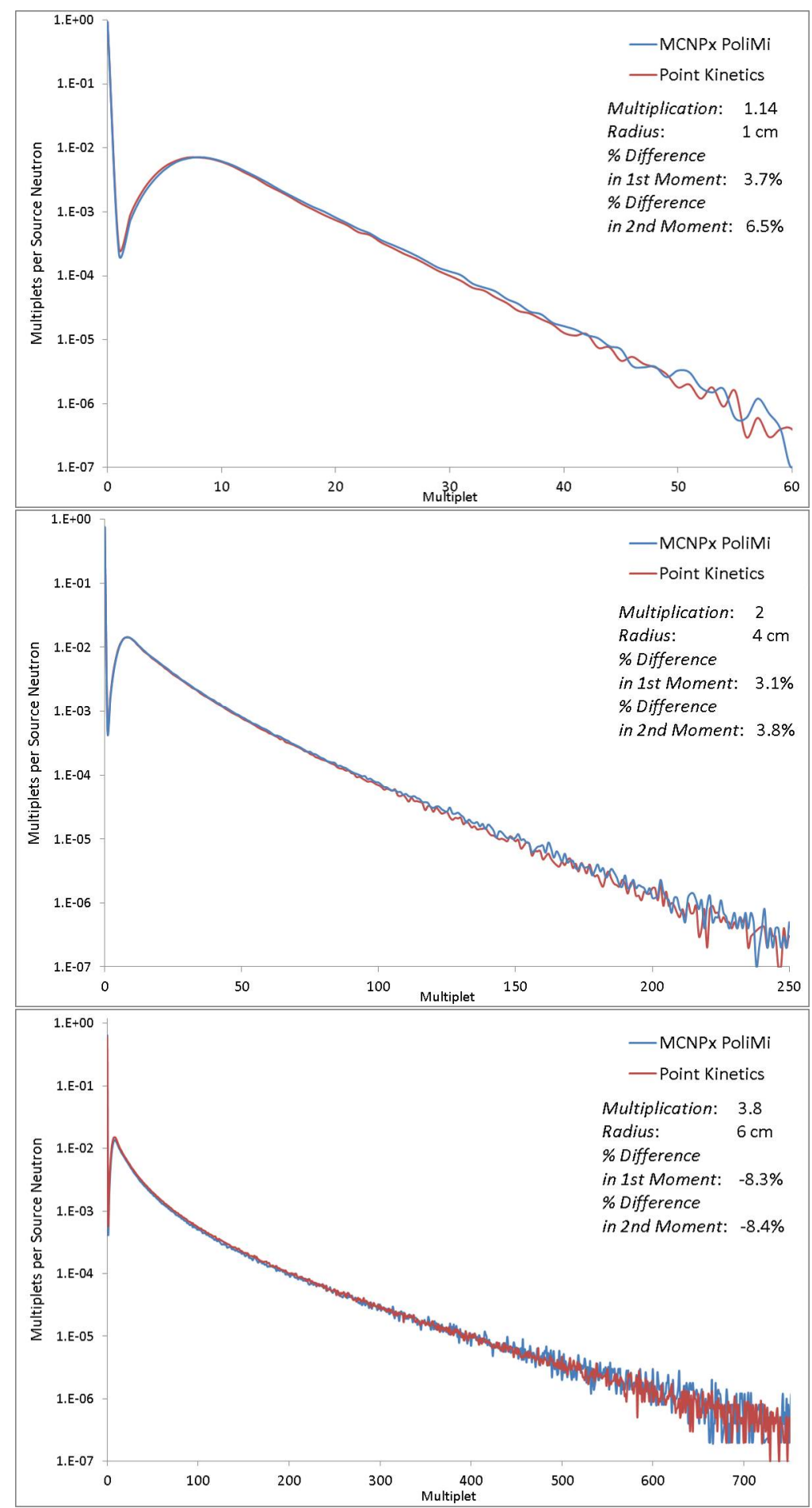

Figure 3.2: Comparison of MCNPx PoliMi fission photons produced to point kinetics model (Equation 3.25) of photons produced within ${ }^{235} U$ metal spheres of 1,4 and $6 \mathrm{~cm}$ in radius respectively. 


\subsection{Composite Equation}

Since the neutron and photon distributions are so closely linked, combining the two distributions into one joint equation is simple, and has been done by $(51 ; 46)$. The composite generating function is now defined as

$$
h(x, y) \equiv \sum_{n=0}^{\infty} y^{n} \sum_{m=0}^{\infty} x^{m} P_{n, m}(p),
$$

where $y$ and $n$ track neutron distributions and $x$ and $m$ track photon distributions. The equivalent equation to Equation 3.8 is then

$$
h(x, y)=(1-p) y+p E(x) C(h(x, y))
$$

Extending this composite formulation to Equation 3.19:

$$
H(x, y)=\left(1-\omega_{f}\right) y+\omega_{f} F(x) D(h(x, y))
$$

Just as before, the probabilities of $n$ neutrons and $m$ photons being emitted from a chain are given by

$$
P_{n, m}^{s}(p)=\left.\frac{1}{n ! m !} \frac{\partial^{n}}{\partial^{n} y}\left(\frac{\partial^{m}}{\partial^{m} x} I(x, y)\right)\right|_{x=0, y=0}
$$

To get neutron distributions from this composite definition, take derivatives only of $y$ and set $x=1$, which is equivalent to taking the zero ${ }^{t h}$ moment of the photon distributions, meaning all distributions are considered.

$$
h(1, y)=(1-p) y+p(E(1)=1) C(h(1, y))=(1-p) y+p C(h(1, y))
$$


and

$$
H(1, y)=\left(1-\omega_{f}\right) y+\omega_{f}(F(1)=1) D(h(1, y))=\left(1-\omega_{f}\right) y+\omega_{f} D(h(1, y))
$$

such that the probabilities become

$$
\left.P_{n}^{s}(p)=\left.\frac{1}{n ! m !} \frac{\partial^{n}}{\partial^{n} y}\left(\frac{\partial^{0}}{\partial^{0} x} H(x, y)\right)\right|_{x=1, y=0}=\frac{1}{n !} \frac{\partial^{n}}{\partial^{n} y} H(1, y)\right)\left.\right|_{y=0}
$$

Equivalently, setting $y=1$ reduces to an equation that gives only the photon distributions.

\subsection{Adding Non-Fission Interactions}

For fission energy neutrons, the interactions in uranium metal are dominated by scattering and fission interactions. Since scattering interactions do not directly affect the number of neutrons escaping a system, the previously described point kinetics equations approximate the neutron population of a uranium metal object well.

Moving away from a system that consists of only fission energy neutrons, other interactions begin to contribute to the balance of particles. Using a source of 14 $\mathrm{MeV}$ neutrons introduces a significantly higher probability of $(n, 2 n)$ interactions occurring. An additional consideration is that ${ }^{238} \mathrm{U}$ has a high rate of $(\mathrm{n}, 2 \mathrm{n})$ and fission interactions induced by $14 \mathrm{MeV}$ neutrons.

Additionally, incorporating photons into these equations requires that other interactions be considered. Photons are attenuated in the dense uranium metal at a very high rate, and neutron inelastic scattering interactions occur frequently as well. Since both of these interactions affect the photon number distribution greatly, 
it is important that they are accounted for in the model.

The following sections describe the incorporation of photon absorption, $14 \mathrm{MeV}$ neutron $(n, 2 n)$ interactions, and neutron inelastic scattering interactions into the previously described model in an effort to model the neutron and photon populations of these systems as closely as possible.

\subsubsection{Energy Group Cross Section Assumptions}

These new interactions are all modeled without consideration for neutron or photon energy in order to maintain the simplistic nature of the model. While the interaction rates of neutrons in uranium metal are very sensitive to neutron energy, a two group energy approximation (14 MeV and fission energy) is reasonable because of how little energy is lost by a neutron before it either interacts or escapes the system (not considering shielding). The average neutron energy lost due to elastic scattering is given by

$$
\Delta E=\frac{(1-a) E}{2}, \quad a=\left(\frac{A-1}{A+1}\right)^{2}
$$

where $\Delta E$ is the average energy lost, $E$ is the initial neutron energy, and $A$ is the mass of the scattering nucleus. Considering the average number of scattering interactions that would occur in $10 \mathrm{~cm}$ of ${ }^{235} \mathrm{U}$ metal, the average energy lost would be $1.9 \%$ for a fission energy neutron and would be $1.2 \%$ for a $14 \mathrm{MeV}$ neutron. This example shows that even for significant travel distances, the total energy loss is quite small. 


\subsubsection{Source Neutron $(\mathrm{N}, 2 \mathrm{~N})$ Interactions}

First the $14 \mathrm{MeV}$ neutron $(n, 2 n)$ interaction will be added as a possible fission chain initiator in the point kinetics model. This can be modeled similar to the $14 \mathrm{MeV}$ fission interaction except for the number of neutrons produced, since for $(\mathrm{n}, 2 \mathrm{n})$ interactions there will always be two neutrons released. Letting $N(x)$ be the generating function for $(\mathrm{n}, 2 \mathrm{n})$ interactions, the probabilities $\alpha_{\nu}^{N}=0$ for all $n \neq 2$ :

$$
N(y)=\sum_{\nu=0}^{N_{c}} \alpha_{\nu}^{N} y^{\nu}=\nu_{2}^{N} y^{2}
$$

Due to normalization, $\nu_{2}^{N}=1$ so that the number distribution function simplifies to $N(y)=y^{2}$. Adding this into the Böhnel equation gives

$$
H(x, y)=\left(1-\omega_{f}-\omega_{n n}\right) y+\omega_{f} F(x) D(h(x, y))+\omega_{n n}(h(x, y))^{2}
$$

where $\omega_{n n}$ is the probability of a $14 \mathrm{MeV}(n, 2 n)$ interaction occurring, and $(h(y))^{2}$ describes two neutrons being produced in the chain, with the ability to escape the system or to create fission interactions.

Figure 3.3 shows the effects of adding the $(n, 2 n)$ interaction into Equation 3.31. It is clear visually that these equations fit the MCNP generated results better than was seen in Figure 3.1. This is further supported by comparing the first and second moments of each distribution, which are closer in Figure 3.3 than in Figure 3.1 for all sizes of uranium sphere. This better fit builds confidence in the addition of the $(\mathrm{n}, 2 \mathrm{n})$ source interaction in Equation 3.31. 


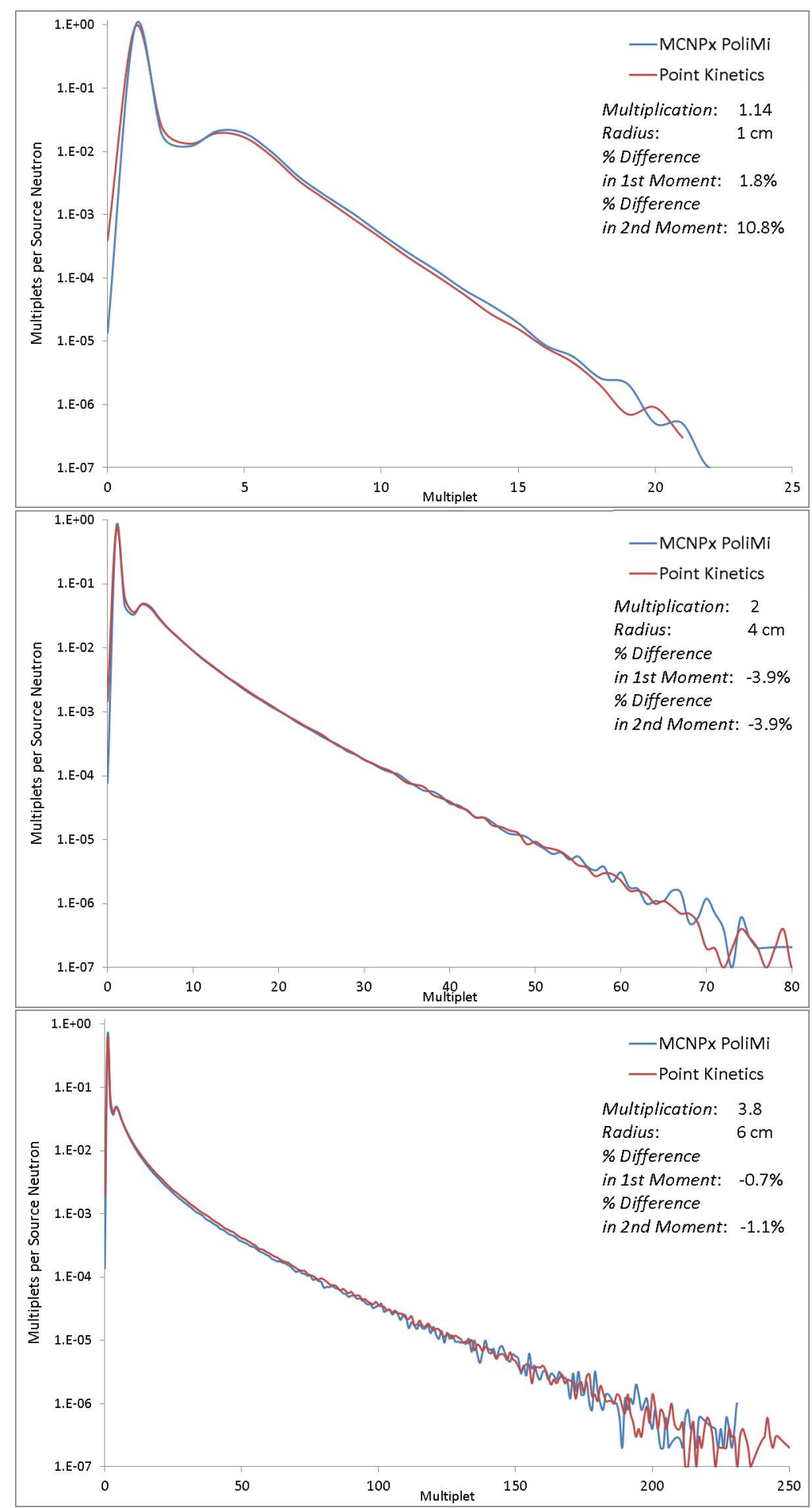

Figure 3.3: Comparison of MCNPx PoliMi output to point kinetics model (Equation 3.31) of neutrons escaping from ${ }^{235} U$ metal spheres of 1,4 and $6 \mathrm{~cm}$ in radius respectively. 


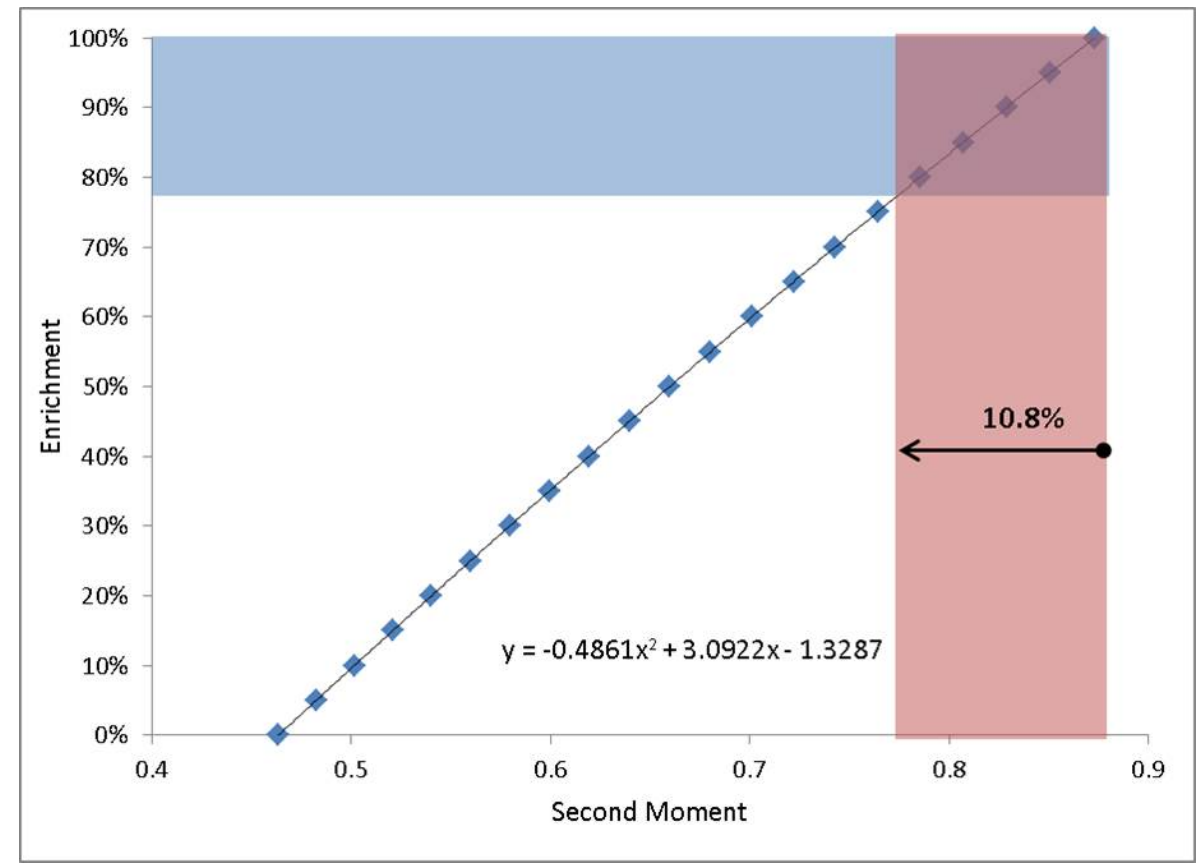

Figure 3.4: Example to illustrate the enrichment determinations in Tables 3.1, with $(\mathrm{n}, 2 \mathrm{n})$ interactions included.

\subsubsection{Predictive Capabilities}

The outputs of these point kinetics equations are obscure since they have not been related to a metric of interest, such as mass or enrichment. Without understanding how these nuclear moments are related to useful values, it is not clear what an acceptable agreement between the model and simulation is. Therefore, a simple exercise is done to translate the percent difference between the point kinetics model and the simulations to the precision level of enrichment prediction.

Figure 3.4 shows how this simple exercise was performed; The enrichment vs second neutron moment for a $1 \mathrm{~cm}$ sphere of ${ }^{2} 35 \mathrm{U}$ is plotted, and a second order polynomial is fit to the point kinetics predicted data points. When comparing simulations to point kinetics results in Figure 3.3, it was determined that the \% difference between the second moment for a $1 \mathrm{~cm}$ uranium sphere was $10.8 \%$. Fig- 
Table 3.1: Point Kinetics Enrichment Prediction from Neutron Distribution

\begin{tabular}{|c|c|}
\hline Point Kinetics Predicted Moments with \% Difference & Predicted Enrichment \\
\hline \multicolumn{2}{|l|}{$1 \mathrm{~cm}$ sphere } \\
\hline First Moment $\pm 1.8 \%$ Difference & $>95 \%$ \\
\hline Second Moment \pm 10.8 \% Difference & $>78 \%$ \\
\hline \multicolumn{2}{|l|}{$4 \mathrm{~cm}$ sphere } \\
\hline First Moment $\pm 3.9 \%$ Difference & $>94 \%$ \\
\hline Second Moment $\pm 3.9 \%$ Difference & $>96 \%$ \\
\hline \multicolumn{2}{|l|}{$6 \mathrm{~cm}$ sphere } \\
\hline First Moment $\pm 0.7 \%$ Difference & $100 \%$ \\
\hline Second Moment $\pm 1.1 \%$ Difference & $100 \%$ \\
\hline
\end{tabular}

ure 3.4 shows how this level of confidence in the neutron moment translates into a confidence in enrichment determination, if the sphere being measured were $100 \%$ ${ }^{235} \mathrm{U}$. Because the photon rate is only known to within $\pm 10.8 \%$, the enrichment of a $100 \%$ enriched uranium sphere could only be determined to be greater than $78 \%$ enriched, which is reflected in Table 3.1.

The study in Table 3.1 provides context to help the reader understand how the closeness of fit shown in Figure 3.3 translates to enrichment agreement. The results show that even if the point kinetics model differs from the "real" distribution by a few percent, it is still able to predict enrichment to within a useful range.

\subsubsection{Photon Absorption}

While the number of photons released per fission is much higher than the number of neutrons, the absorption of photons in the highly dense uranium metal is much more severe than for neutrons. In fact, neutron absorption is so minor that it is not considered in this model, though others have included it in the past (53). As described in reference (53), one way to model photon absorption is by inserting the photon non-absorption probability into the point kinetics model as a binary 
function. Each photon will either escape the system or be absorbed:

$$
l(x)=\gamma x+(1-\gamma)
$$

where $\gamma$ is the probability of non-absorption of an average photon. Equation 3.27 then becomes

$$
h(l(x), y)=(1-p) y+p E(l(x)) C(h(l(x), y))
$$

The fission photon distribution function including absorption, $E(l(x))$, then becomes

$$
E(l(x))=\sum_{\nu=0}^{N_{c}} \alpha_{\nu}^{E}(\gamma x+(1-\gamma))^{\nu}
$$

Taking the first moment of this function:

$$
\left.\frac{\partial}{\partial x} E(l(x))\right|_{x=1}=\frac{\partial}{\partial x} \sum_{\nu=1}^{N_{c}} \alpha_{\nu}^{E} \gamma(\gamma x+(1-\gamma))^{\nu}=\gamma \sum_{\nu=1}^{N_{c}} \alpha_{\nu}^{E}=\gamma \nu_{1}^{E}
$$

It is easy to see from this derivation that all of the moments of the photon nuclear data functions can be changed in the following way to include photon absorption:

$$
\nu_{n} \rightarrow \gamma^{n} \nu_{n}
$$

For thicker objects with high photon attenuation, it is clear that higher order photons will be severely reduced.

Figure 3.5 shows a comparison of Equation 3.28 (or 3.31) with photon absorption incorporated. Unlike in Figure 3.2, the MCNPx PoliMi tally in Figure 3.5 is a tally of all photons escaping the spheres. In the MCNPx PoliMi simulations, other interactions besides fission are producing photons, some of which are escaping the 

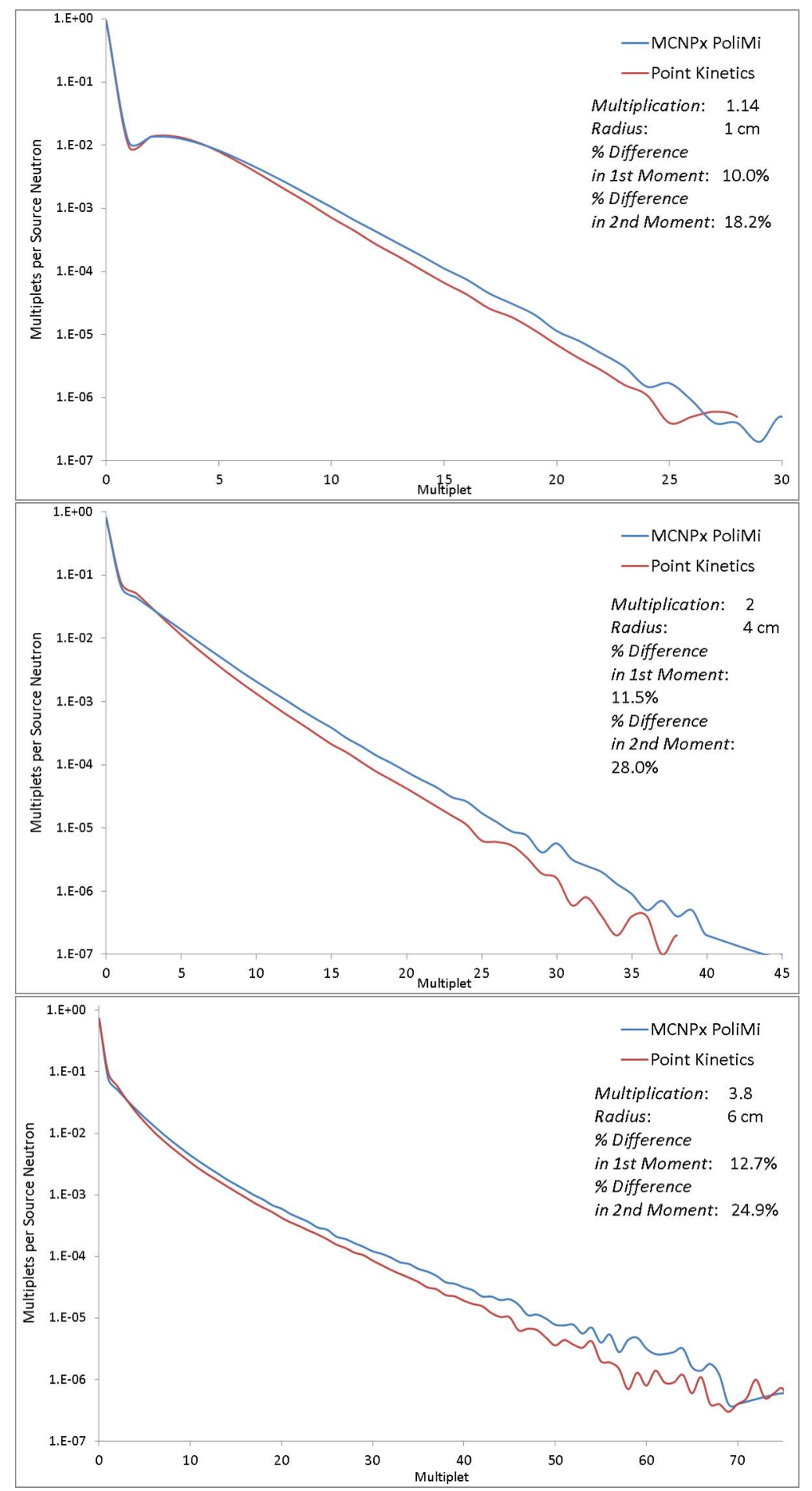

Figure 3.5: Comparison of MCNPx PoliMi output to point kinetics model (Equation 3.25 with absorption incorporated) of photons escaping from ${ }^{235} U$ metal spheres of 1,4 and $6 \mathrm{~cm}$ in radius respectively. The consistent under-prediction of the point kinetics distributions is expected because inelastic scattering gamma-rays have not yet been modeled. 
sphere. Therefore, the degree of match is not expected to be perfect; it makes sense that the point kinetics equations with only fission photons and absorption included consistently under predicts the MCNPx PoliMi output. It turns out that most of the non-fission photons seen in the MCNPx PoliMi output are from inelastic scattering interactions, which is incorporated in the next section.

\subsubsection{Inelastic Scattering}

Inelastic scattering will be included in point kinetics model both for $14 \mathrm{MeV}$ and fission energy neutrons separately. Because higher energy neutrons will inherently lead to different gamma-ray cascades, there needs to be separate functions to describe the resulting gamma-ray distribution for each neutron energy group. Let $M(x)$ represent the gamma-ray distribution induced by inelastic scattering interactions with fission energy neutrons such that,

$$
M(x)=\sum_{\nu=1}^{N_{c}} \alpha_{\nu}^{M} x^{\nu}
$$

Then let $J(x)$ be the counterpart for inelastic scattering gamma-rays induced by $14 \mathrm{MeV}$ neutrons. Note that $\alpha_{0}^{M}$ and $\alpha_{0}^{J}$ are both set to zero, because a scattering interaction that produces zero photons would be elastic, and elastic scattering is not modeled here.

Inelastic scattering is modeled as an interaction that produces one neutron and some distribution of photons, described by the functions described previously. The function $h(l(x), y)$ now becomes

$$
h(l(x), y)=(1-p)\left(1-p_{n g}+p_{n g} M(l(x))\right) y+p E(l(x)) C(h(l(x), y))
$$


and similarly $H(l(x), y)$ becomes

$H(l(x), y)=\left(1-\omega_{f}-\omega_{n n}\right)\left(1-\omega_{n g}+\omega_{n g} J(l(x))\right) y+\omega_{f} F(l(x)) D\left(h(l(x), y)+\omega_{n n}(h(l(x), y))^{2}\right.$

where $p_{n g}$ and $\omega_{n g}$ are the probabilities of inelastic scattering gamma-rays for fission energy and $14 \mathrm{MeV}$ neutrons, respectively. The first few moments with with respect to the photon distributions are:

$$
\begin{aligned}
\Psi_{x}= & \left(1-\omega_{f}-\omega_{n n}\right) \omega_{n g} \nu_{1}^{J} \gamma+\omega_{f} \nu_{1}^{F} \gamma+\phi_{x}\left(\omega_{f} \nu_{1}^{D}+2 \omega_{n n}\right) \\
\phi_{x}= & \frac{(1-p) p_{n g} \nu_{1}^{M} \gamma+p \nu_{1}^{E} \gamma}{1-p n u_{1}^{C}} \\
\Psi_{x x}= & \left(1-\omega_{f}-\omega_{n n}\right) \omega_{n g} \nu_{2}^{J} \gamma^{2}+\omega_{f} \nu_{2}^{F} \gamma^{2}+\left(\omega_{f} \nu_{1}^{F} \nu_{1}^{D} \gamma+\omega_{n n}\right) \phi_{x}+ \\
& \omega_{f} \nu_{2}^{D}\left(\phi_{x}\right)^{2}+\left(\omega_{f} \nu_{1}^{D}+2 \omega_{n n}\right) \phi_{x x} \\
\phi_{x x}= & \frac{(1-p) p_{n g} \nu_{2}^{M} \gamma^{2}+p\left(\nu_{2}^{E} \gamma^{2}+\nu_{1}^{E} \nu_{1}^{C} \gamma \phi_{x}+\nu_{2}^{C}\left(\phi_{x}\right)^{2}\right.}{1-\nu_{1}^{C} p}
\end{aligned}
$$

There could be other methods of modeling the inelastic scattering interaction, such as allowing the scattered neutrons to re-enter the system as opposed to escaping. This model was deemed beneficial due to the fact that the neutron-only moments are unaffected by the interaction. If $x=1$, the inelastic scattering interaction drops out of the equations completely, and the neutron-only moments are the same as before.

Figure 3.6 shows a comparison of Equation 3.36 to MCNPx PoliMi simulations. Again, the MCNP tally in Figure 3.6 is a tally of all photons escaping the spheres (the same tally plotted in Figure 3.5). However, it is clear that adding inelastic scattering gamma-rays into the point kinetics equations gives a significantly closer match to the MCNP tally. This can be seen visually and also by the smaller difference 


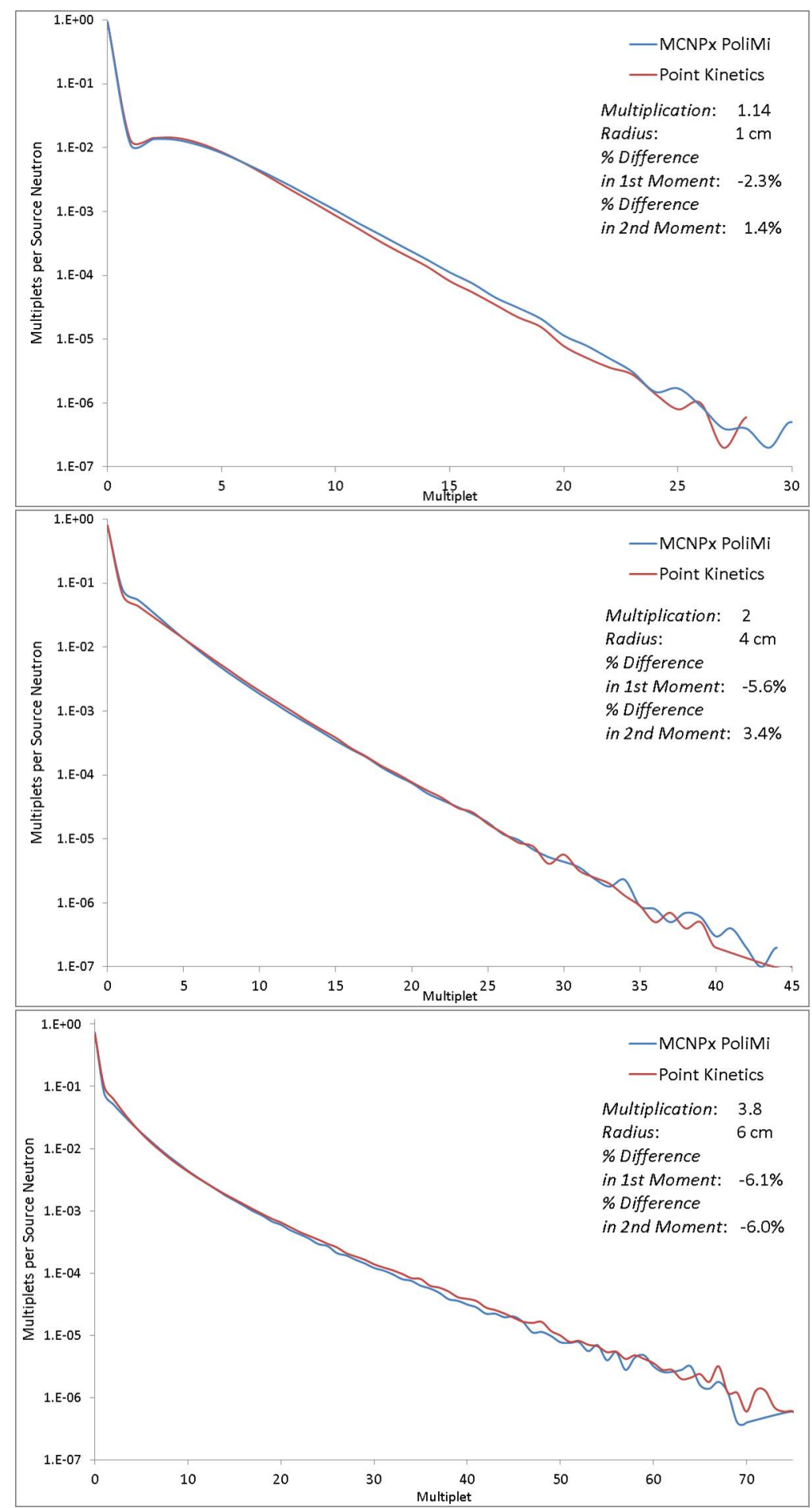

Figure 3.6: Comparison of MCNPx PoliMi output to point kinetics model (Equation 3.36) of photons escaping from ${ }^{235} U$ metal spheres of 1,4 and $6 \mathrm{~cm}$ in radius respectively. 
between the first and second moments.

While this is a significantly closer fit than seen in Figure 3.5, some of the simplifying assumptions lead to the point kinetics matching less well for larger, higher multiplicity objects in comparison to smaller objects. This is partly due to the assumption that the fissions are distributed equally throughout the sphere. Since the neutron leakage probability is lower towards the center of the sphere, the fission probability is slightly higher in the center of the sphere, leading to slightly more fissions and inelastic scatters occurring in the middle of the sphere than towards the edges. However, the point model inherently assumes an average fission probability, leading to slight inaccuracies (54). Since photons are highly attenuated by dense uranium metal, the photons being produced at a higher rate in the center of the sphere leads to a higher level of attenuation. This is why in the $6 \mathrm{~cm}$ radius sphere results in Figure 3.6, the point kinetics clearly over predicts the MCNP results. However, this is a slight affect, and the point kinetics is still a very good representation of the multiplying system resembling a sphere.

\subsubsection{Predictive Capabilities}

Just as with the neutron distributions, without understanding how these nuclear moments relate to useful values, it is not clear what an acceptable agreement between the model and simulation is. Table 3.2 shows how the closeness of fit between MCNP and point kinetics output first and second photon moments translates to a range of enrichment levels, just as was done in Section 3.6.3. The percent differences in the moments, given in Figure 3.6, were taken as the \% difference to show how this confidence level would translate to a range of predicted enrichment. The purpose of Table 3.2 is to show that even if the point kinetics model differs 
Table 3.2: Point Kinetics Enrichment Prediction from Photon Distribution

\begin{tabular}{|c|c|}
\hline Point Kinetics Predicted Moments with \% Difference & Predicted Enrichment \\
\hline \multicolumn{2}{|l|}{$1 \mathrm{~cm}$ sphere } \\
\hline First Moment $\pm 2.3 \%$ Difference & $>95 \%$ \\
\hline Second Moment $\pm 1.4 \%$ Difference & $>98 \%$ \\
\hline \multicolumn{2}{|l|}{$4 \mathrm{~cm}$ sphere } \\
\hline First Moment $\pm 5.6 \%$ Difference & $>94 \%$ \\
\hline Second Moment $\pm 3.4 \%$ Difference & $>98 \%$ \\
\hline \multicolumn{2}{|l|}{$6 \mathrm{~cm}$ sphere } \\
\hline First Moment $\pm 6.1 \%$ Difference & $>96 \%$ \\
\hline Second Moment $\pm 6 \%$ Difference & $>99 \%$ \\
\hline
\end{tabular}

from the "real" distribution by a few percent, it is still able to predict enrichment to within a useful range. The $6 \mathrm{~cm}$ uranium sphere results are an especially good example of this; the $6 \%$ uncertainties of the first and second photon moments still lead to predictions of greater than $96 \%$ enriched uranium.

\subsubsection{Determining Interaction Probabilities}

Adding interactions into this model means adding more unknown terms. However, based on our assumptions of neutron energy groups (fission energy and 14 $\mathrm{MeV}$ ), these additional probabilities can be estimated by using basic cross section data. Each neutron is produced at some location in the material (call it $z=0$ ), and the probability of a neutron interacting per unit length traveled is given by

$$
\text { Prob. of Int. }=\Sigma_{I} e^{-\Sigma_{R} z} d z
$$

where $\Sigma_{I}$ is some interaction cross section and $\Sigma_{R}$ is the removal cross section. Since the probability of fission, $p$, is directly related to the multiplication of the system, which is the desired output parameter, it would be useful to get the other interaction probabilities in terms of $p$. Let $f_{I}$ be the ratio of the probability of some 
interaction to the probability of fission, which would reduce to:

$$
f_{I}=\frac{p_{I}}{p}=\frac{\Sigma_{I} e^{-\Sigma_{R} z} d z}{\Sigma_{f} e^{-\Sigma_{R} z} d z}=\frac{\sigma_{I}}{\sigma_{f}}
$$

where the $\sigma$ values refer to the microscopic cross sections of the given material, since the density of the material can be divided out of the fraction. The factor $f_{I}$ can then be taken as a known quantity, reducing the number of unknowns. The two equations, describing chain initiation and chain propagation, then become

$$
\begin{aligned}
h(l(x), y)= & \left(1-p\left(1+f_{n g}-f_{n g} M(l(x))\right)\right) y+p E(l(x)) C(h(l(x), y)) \\
H(l(x), y)= & \left(1-\omega_{f}\left(1+f_{n n}^{s}+f_{n g}^{s}-f_{n g}^{s} J(l(x))\right)\right) y+ \\
& \alpha_{f} F(l(x)) D(h(l(x)), y)+\omega_{f} f_{n n}^{s} N(h(l(x), y))
\end{aligned}
$$

where the $s$ superscript denotes the use of source energy cross sections. The problem with this approach comes with its sensitivity to neutron energy. As fission energy neutrons lose energy, the value of $\sigma_{f}$ changes significantly, and other interaction cross sections do not change in the same way, meaning that the factors $f_{I}$ are very sensitive to energy. The high energy source neutrons are slightly more resistant to this sensitivity, because the fission cross section doesn't change as significantly for ${ }^{235} \mathrm{U}$ at high neutron energies. However, as discussed previously, the neutron energy does not change greatly as it travels through uranium metal, meaning that this factor gives a good estimate of the interaction probabilities. 


\subsubsection{Coupling Constant, $\omega_{f}$}

The last equation that will be explicitly calculated is $\omega_{f}$, the probability of a 14 $\mathrm{MeV}$ induced fission. This can again be calculated with Equation 3.41 using known nuclear data and the thickness of the fissile object. The thickness of the fissile object can be determined by radiography.

\subsubsection{Photon Absorption Probability}

It is not straight forward to model the photon absorption because it is dependent on where the fission interactions are occurring in the system, and the average length of material between that fission location and the surface of the material. An intuitive approach was attempted to calculate the photon absorption probability for a given object. The approach used was a basic exponential attenuation function,

$$
l=e^{-\sigma \rho f(x)}
$$

where the attenuation coefficient $\sigma$ was extracted from MCNP, and the average distance out of the material, is some function of the geometry $f(x)$. The geometry function used for uranium spheres is described in Appendix C, and gave good results when used to calculate $\omega_{f}$ for $14 \mathrm{MeV}$ sources distributed in a sphere. However, it was found when comparing to simulations that this calculation of photon nonabsorption was not a good estimate. There are a few reasons why this may be the case:

- The geometry function used estimates the distance traveled by one photon that is equally likely to travel in any direction out of a sphere from the point of origin. The reality is that several photons are released at a time, which puts 
constraints on the direction the photons can travel, and the energies of the emerging photons.

- A exponential function describes the attenuation of a mono-energetic photon flux in a material, but the photons here have a range of energies.

- The location of the fissions within the object is not entirely uniform. Fission interactions are slightly more likely to be induced in locations where fission neutrons are less likely to escape (the center of the object). This issue of multiplication being spatially dependent is discussed by Hauck and Henzl (54)

- The average path length of fission photons in dense uranium metal is small. This means that these minor effects can cause large differences.

Given these issues, the absorption probability values used were determined empirically by running MCNP simulations and tallying the number of absorptions and the total photons produced.

\subsection{Adding Shielding}

A major motivation of this dissertation is looking at ways that photon signatures can complement neutron signatures, especially when a neutron attenuating material is present. Therefore, this section focuses on polyethylene.

Polyethylene happens to have advantageous physical properties for modeling photons escaping from fission. The material has a very low photon attenuation rate, and the inelastic scattering cross section for both fission energy and $14 \mathrm{MeV}$ neutrons is low enough to be ignored.

The complication is the change in neutron energy of the system. Some of the assumptions made previously relied on the fact that neutrons do not lose significant 
energy before leaving the system. With the addition of polyethylene, the neutrons can lose significantly more energy per scattering interaction. However, this will not be modeled because the main goal of this model is to remain simple. We do not expect the neutron distribution to be well modeled, and will simplify down to photon-only point kinetics equations. However, the photon multiplets are still dependent on the neutrons to propagate the fission chain, so neutron nuclear data is still present in the point kinetics equations.

In order to account for absorption of the photons in the shielding material, the photon non-absorption probability is again modeled as a binary function. Remembering the previous equation for the probability of photons escaping the uranium object:

$$
l(x)=\gamma x+(1-\gamma)
$$

where $\gamma$ is the probability that the photons are NOT absorbed in the uranium. The photons that escape the uranium will then have some probability, $\lambda$ of also escaping the polyethylene, described by the function $j(x)$ :

$$
j(l(x))=\lambda l(x)+(1-\lambda)=(\lambda \gamma) x+(1-(\lambda \gamma))
$$

The results of this derivation show that accounting for photon absorption in shielding material is as simple as replacing all values of $\gamma$ in Equations 3.38-3.40 with $\gamma \times \lambda$

One additional tricky aspect of adding shielding, especially a low-Z shielding, is that the multiplication of the system changes significantly. The $4 \mathrm{~cm}$ sphere of uranium modeled previously had a multiplication value of 2 , but with $5 \mathrm{~cm}$ of shielding added, the multiplication goes up to 2.6. 


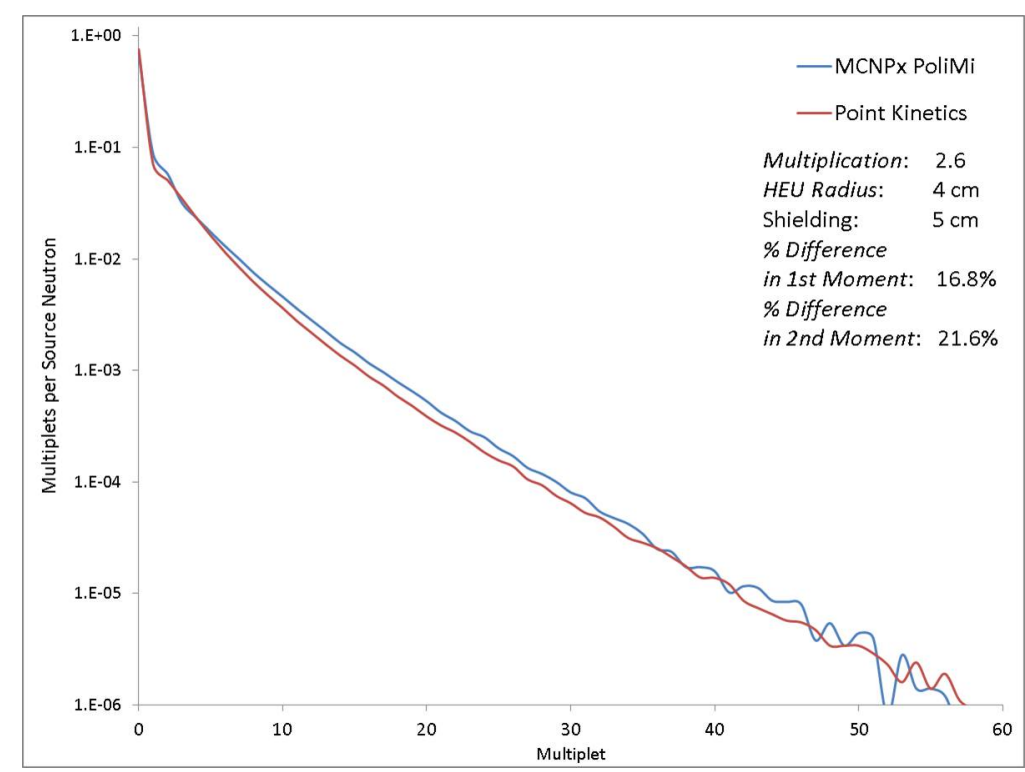

Figure 3.7: Comparison of MCNPx PoliMi output to point kinetics model (Equation 3.36) of photons escaping from a ${ }^{235} \mathrm{U}$ metal sphere $4 \mathrm{~cm}$ radius with $5 \mathrm{~cm}$ of polyethylene shielding.

\subsubsection{Compare Point Kinetics to MCNPx PoliMi Simulations}

Figure 3.7 shows the photon multiplets escaping a $4 \mathrm{~cm}$ radius sphere of $100 \%$ ${ }^{235} \mathrm{U}$ shielded by $5 \mathrm{~cm}$ of polyethylene shielding. As mentioned, the shielding increases the multiplication of the system from 2.0 to 2.6. The non-absorption probability of photons traveling through $5 \mathrm{~cm}$ of polyethylene was determined by MCNPx to be $98 \%$, meaning that the model was not significantly changed from the results seen in Figure 3.6, except for an increased probability of fission, $p$, due to neutron reflection (determined by $M$ ).

Visually, the results are quite similar, especially given the large effect the shielding has on the neutron population, which is not considered in the point kinetics model. While the first and second moments are not as closely predicted by the point kinetics as in the unshielded case, this is still quite impressive given the complexity of the system. Figure 3.8 shows the neutron multiplets, just to show how 


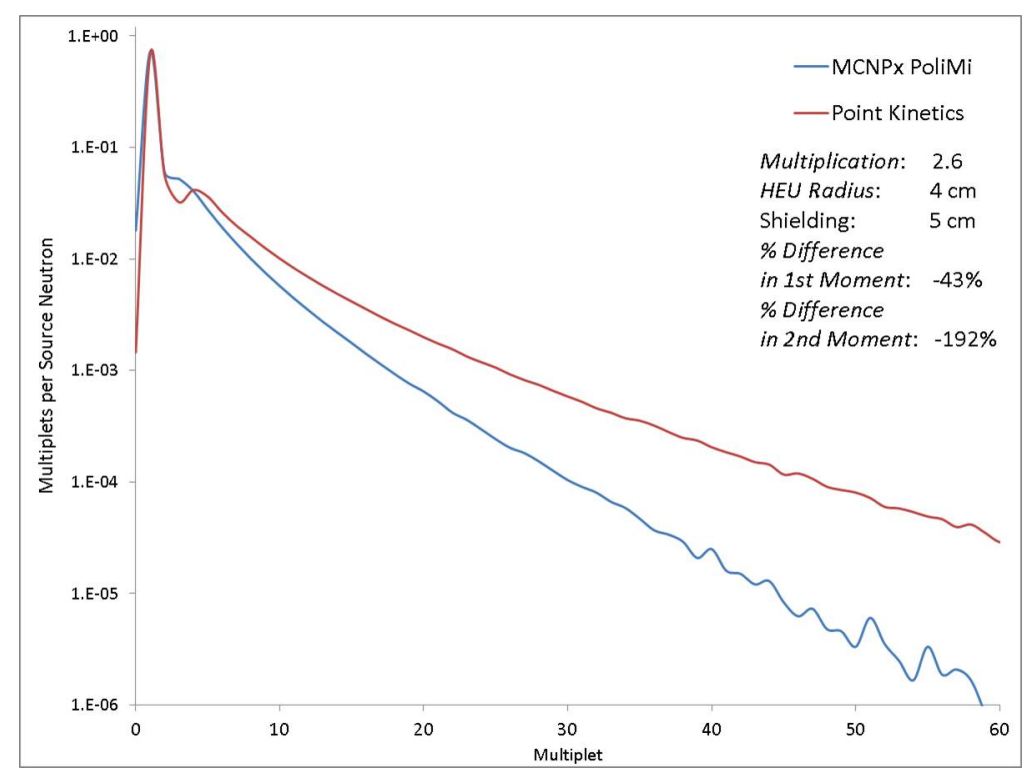

Figure 3.8: Comparison of MCNPx PoliMi output to point kinetics model (Equation 3.36) of neutrons escaping from a ${ }^{235} \mathrm{U}$ metal sphere $4 \mathrm{~cm}$ radius with $5 \mathrm{~cm}$ of polyethylene shielding. The goal of including this plot is to demonstrate the lack of fit.

poorly the point kinetics model does for neutrons.

Since $5 \mathrm{~cm}$ of shielding gave no significant photon attenuation (only $2 \%$ ), the amount of shielding was also doubled to see how well the point kinetics compares with MCNP when photon absorption in polyethylene is affecting the signature (the absorption in $10 \mathrm{~cm}$ of shielding surrounding a $4 \mathrm{~cm}$ sphere is 16\%). Figure 3.9 shows the results. The fit is somewhat worse than that seen in Figure 3.7, but still surprisingly good considering the high level of shielding.

As a way of determining how good the photon absorption in polyethylene is modeled, Figure 3.10 shows the same results from Figure 3.9 but without photon absorption in the polyethylene shielding material. Visually, the comparison is clearly worse in Figure 3.10 than in Figure 3.9, providing confidence in the method used to model photon absorption in shielding material. However, it is interesting to note that the comparison between the first and second moments is actually better 


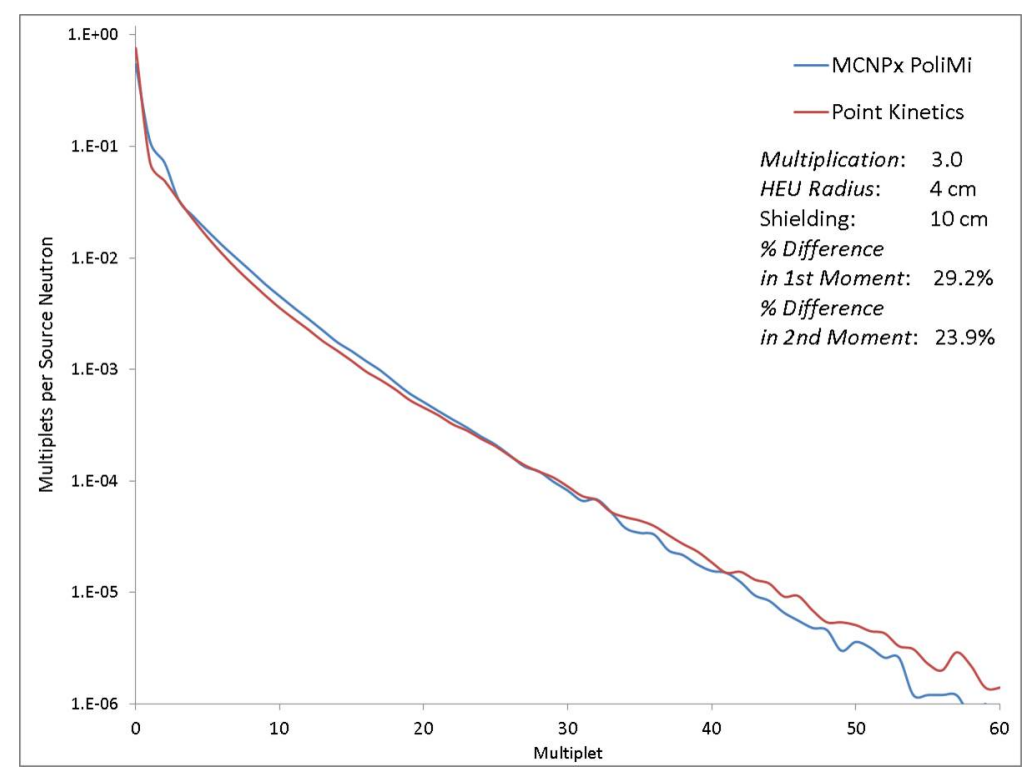

Figure 3.9: Comparison of MCNPx PoliMi output to point kinetics model (Equation 3.36) of photons escaping from a ${ }^{235} \mathrm{U}$ metal sphere $4 \mathrm{~cm}$ radius with $10 \mathrm{~cm}$ of polyethylene shielding.

without the photon absorption modeled. A closer look at the figure shows that the under-prediction in low multiplets cancels out the over-prediction by point kinetics in the high multiplets. This means that the closer fit between the first and second moment is not a sign of a well modeled system, and shows the merit of investigating the full span of multiplets for more complete understanding.

As the multiplication of the system goes up, the fission chains get longer, leading to a more significant sensitivity to neutron energy and population. Therefore, a higher multiplication sphere was also investigated. Figure 3.11 shows the results of comparing MCNP and point kinetics predictions of a $6 \mathrm{~cm}$ sphere of ${ }^{235} \mathrm{U}$ shielded by $5 \mathrm{~cm}$ of polyethylene. Visually, the match between the two predictions does vary, though still not drastically. The moments compare surprisingly closely. 


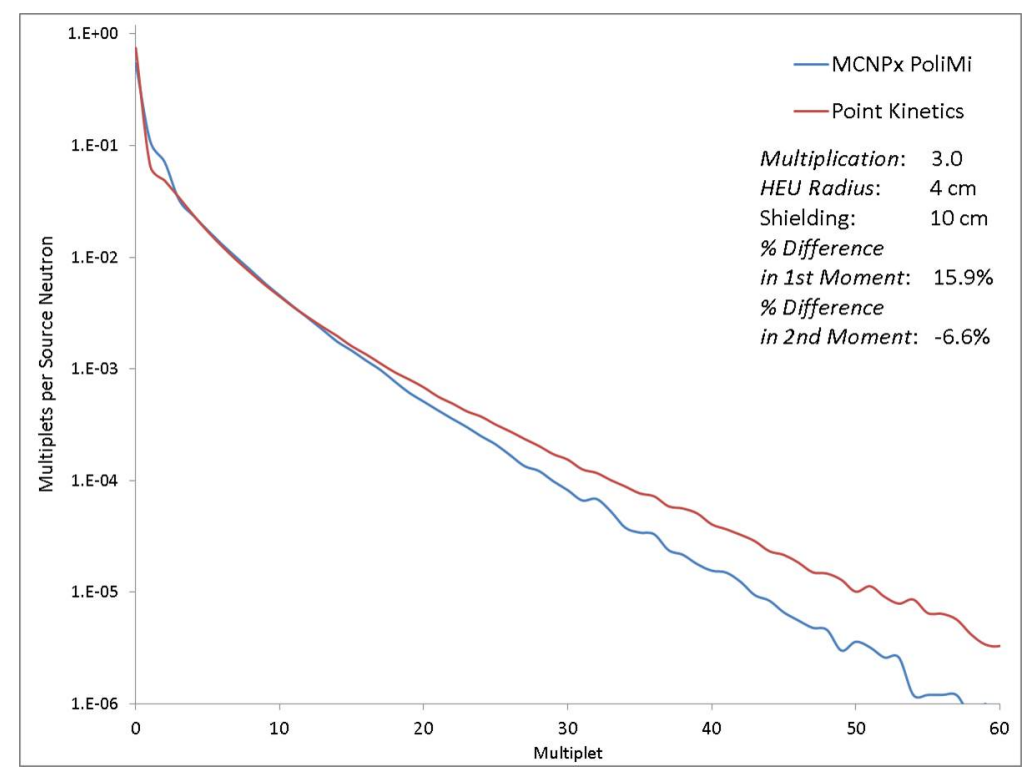

Figure 3.10: Comparison of MCNPx PoliMi output to point kinetics model (Equation 3.36) of photons escaping from a ${ }^{235} \mathrm{U}$ metal sphere $4 \mathrm{~cm}$ radius with $10 \mathrm{~cm}$ of polyethylene shielding without photon absorption in the polyethylene modeled. The lack of fit is expected due to the incomplete model.

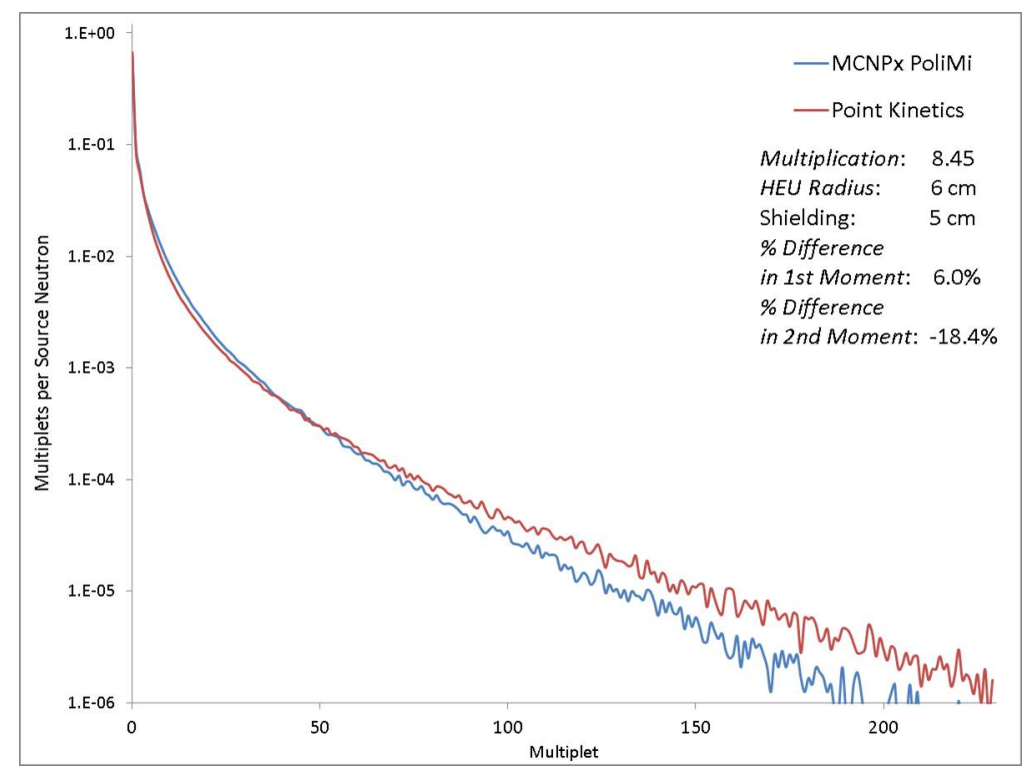

Figure 3.11: Comparison of MCNPx PoliMi output to point kinetics model (Equation 3.36) of photons escaping from a ${ }^{235} \mathrm{U}$ metal sphere $6 \mathrm{~cm}$ radius with $5 \mathrm{~cm}$ of polyethylene shielding. 


\subsection{Modeling Detection Efficiency}

In order for this analytical model to be useful, the detector effects also need to be factored into the final equations. Typically an array of fast plastic or fast liquid scintillators would be used in order to differentiate the prompt photons, source neutrons, and fission neutrons. More detectors lead to less dead time effects; for example, for a detector system with only 8 detectors, one detector being deadened leads to an instantaneous reduction in efficiency of around $12.5 \%$, whereas a system with 80 detectors would only suffer an instantaneous detection efficiency drop of about $1.25 \%$. However, a large number of small detectors can also lead to issues with cross talk, though cross talk can be corrected for in algorithms that sort the data by rejecting pulses from adjacent detectors. Higher multiplication objects with many particles escaping the system at a time will also be more difficult to measure in an array of few detectors, since the dead time length is on the order of a subcritical fission chain length. Since there is no time component in these models, dead time is very difficult to model. Some recent methods to account for dead time is presented by Croft and Favalli (55) and by Hauck et al (56).

Setting aside dead time effects, the detector efficiency $\epsilon$ can be incorporated into the model as a binomial distribution:

$$
e_{m, n}\left(\epsilon_{x}, \epsilon_{y}, p\right)=\sum_{\nu_{x}=m}^{\infty} \sum_{\nu_{y}=n}^{\infty} P_{\nu_{x}, \nu_{y}}^{s}\left(\begin{array}{c}
\nu_{x} \\
m
\end{array}\right)\left(\epsilon_{x}\right)^{m}\left(1-\epsilon_{x}\right)^{\nu_{x}-m}\left(\begin{array}{c}
\nu_{y} \\
n
\end{array}\right)\left(\epsilon_{y}\right)^{n}\left(1-\epsilon_{y}\right)^{\nu_{y}-n}
$$

where each term of the sum is the probability of simultaneously detecting $n$ out of $\nu_{y}$ neutrons emitted and $m$ out of $\nu_{x}$ photons emitted, and the sum of these, called 
$e_{n, m}$, gives the overall the probability of detecting $n$ neutrons and $m$ photons.

$$
e_{m, n}=\sum_{\nu_{x}=m}^{\infty} \sum_{\nu_{y}=n}^{\infty} P_{\nu_{x}, \nu_{y}}^{s} \sum_{j=n}^{\infty}\left(\begin{array}{c}
\nu_{x} \\
j
\end{array}\right)\left(\begin{array}{c}
j \\
m
\end{array}\right)\left(\epsilon_{x}\right)^{j}\left(1-\epsilon_{x}\right)^{\nu_{x}-j} \sum_{i=n}^{\infty}\left(\begin{array}{c}
\nu_{y} \\
i
\end{array}\right)\left(\begin{array}{c}
i \\
n
\end{array}\right)\left(\epsilon_{y}\right)^{i}\left(1-\epsilon_{y}\right)^{\nu_{y}-i}
$$

This of course can be collapsed to detecting either just photons or just neutrons. However, if dead time were to be taken into account, these could not be decoupled, because particles emitted will be deadening detectors and changing the detection efficiency for the system. This highly complex scenario is beyond the scope of this work, primarily because it is very reliant on a timing component that is not modeled in this point kinetics formulation. Using the definition of the moments, given by Equation 3.14 and the following identity,

$$
\sum_{j=q}^{\nu}\left(\begin{array}{l}
\nu \\
j
\end{array}\right)\left(\begin{array}{l}
j \\
q
\end{array}\right) a^{j}(1-a)^{\nu-j}=\left(\begin{array}{l}
\nu \\
q
\end{array}\right) a^{q}
$$

the moments can be simplified in the following way:

$$
e_{m, n}=\epsilon^{m} \epsilon^{n} \Omega_{m, n}
$$

\subsubsection{Compare Point Kinetics to MCNPx PoliMi Simulations}

Now that detector efficiency has been added into our analytical model, these final results can be compared to MCNPx PoliMi simulations with detectors added. This should give an idea of how realistically the analytical model can represent a detector system measuring these signatures. A MCNPx PoliMi simulation was created with 18 detectors with $10 \times 10 \mathrm{~cm}^{2}$ detector faces located $20 \mathrm{~cm}$ away from the center of the object being measured. The detector system was determined to have about 33\% efficiency for both fission neutrons and photons. A visual of this 


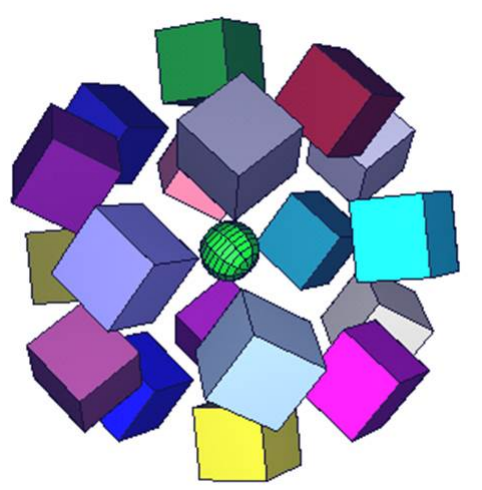

Figure 3.12: The detector setup used for simulations with 18 total $10 \times 10 \mathrm{~cm}^{2}$ detectors

modeled setup is shown in Figure 3.12

The simulation is still not realistic of an active interrogation detector system measurement. The $14 \mathrm{MeV}$ source is still evenly distributed throughout the material. Each simulated detector is also encased on all sides in a "zero importance" material, so that cross talk is severely reduced since it can only occur from the front detector face. The detectors were also modeled to have infinite dead time, which is not far from reality in the type of prompt detection system that this study is limited to.

Again, simulations of $1 \mathrm{~cm}, 4 \mathrm{~cm}$, and $6 \mathrm{~cm}$ spheres of ${ }^{235} \mathrm{U}$ metal were modeled. The slight increase in multiplication due to neutrons reflecting off of the detector system was accounted for, though the effect is very minor. The resulting comparison between simulation and point kinetics results for neutrons and photons can be seen in Figures 3.13 and 3.14.

The results are a significant deviation from the close match that has been seen previously. This is primarily due to lack of dead time modeled in the point kinetics equations as well as a significant level of cross-talk. The dead time effects are especially evident in the highest multiplication $6 \mathrm{~cm}$ radius sphere, which clearly 


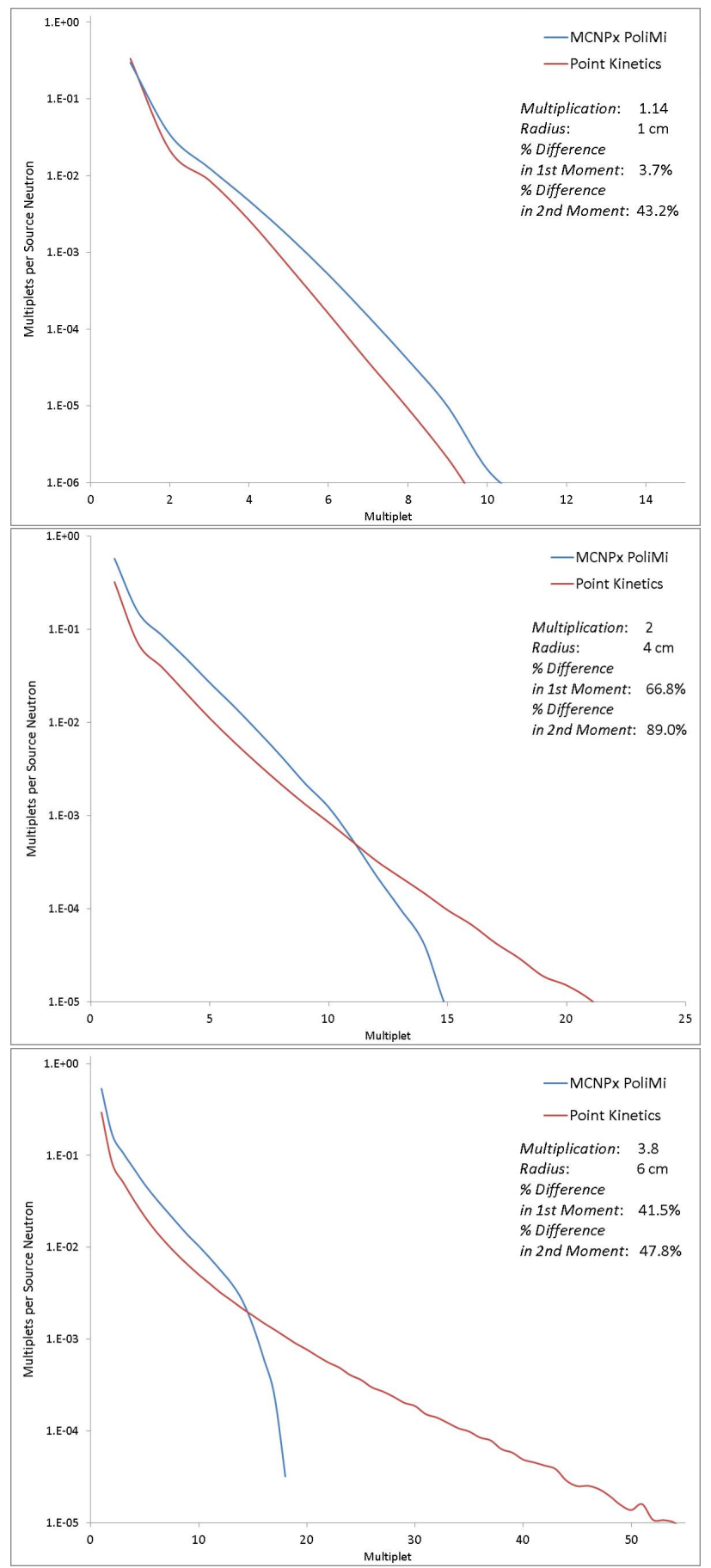

Figure 3.13: Comparison of MCNPx PoliMi output to point kinetics model (Equation 3.46) of neutrons escaping from ${ }^{235} \mathrm{U}$ metal spheres of 1,4 and $6 \mathrm{~cm}$ in radius respectively. 

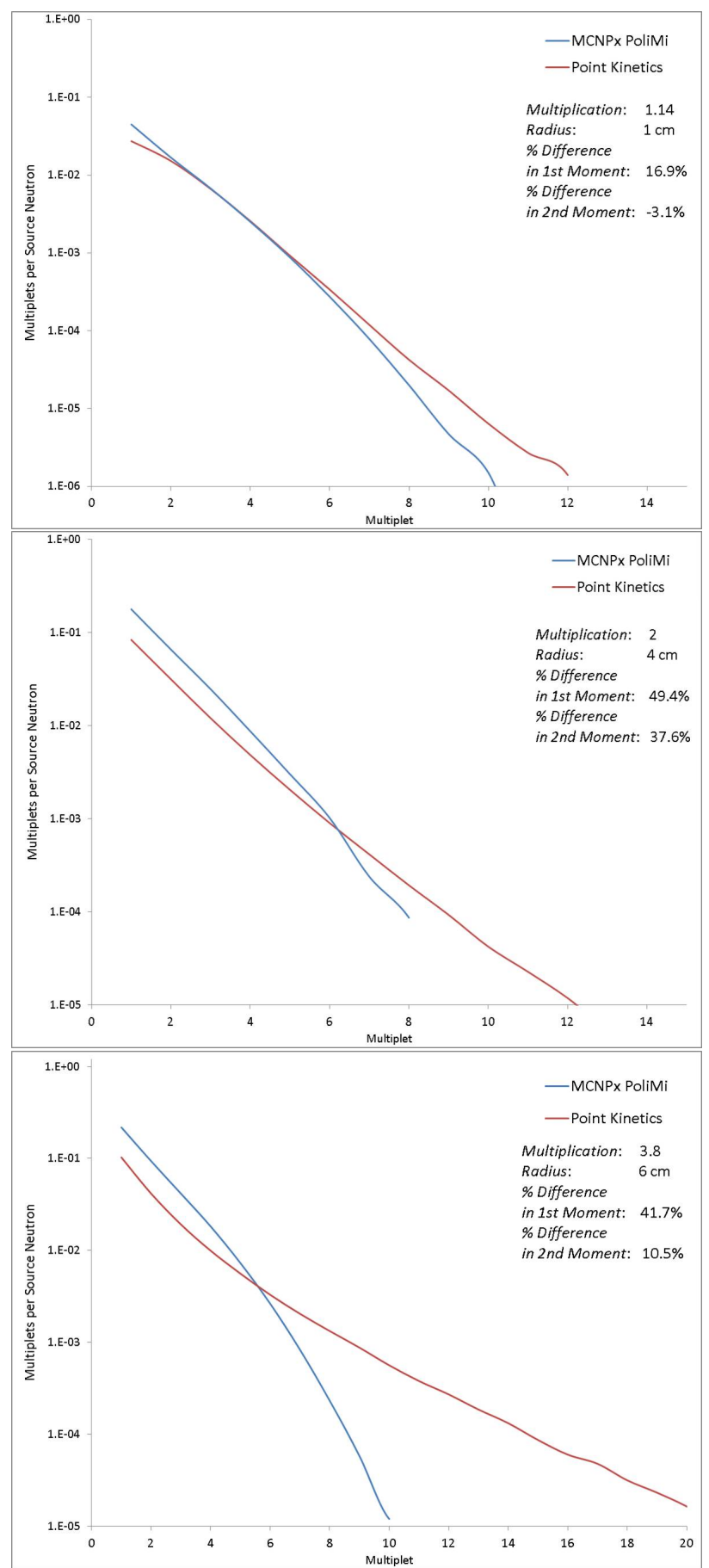

Figure 3.14: Comparison of MCNPx PoliMi output to point kinetics model (Equation 3.46) of photons escaping from ${ }^{235} \mathrm{U}$ metal spheres of 1,4 and $6 \mathrm{~cm}$ in radius respectively. 
suffers from having a maximum of 18 particles detected in the system at a time.

The cross-talk was investigated by simulating a single $2 \mathrm{MeV}$ neutron source in the middle of the detector system. Even with the "blinders" between the detectors, $10 \%$ of the detections resulted in cross-talk, and $2 \%$ of detections were registered in 3 detectors or more. The neutron source also created photons in the detector, which was then detected in multiple detectors, which is an additional troublesome crosstalk effect. This issue could partially be resolved by using a detector array that is on a 2-D plane that also includes cross-talk rejection in the signal processing algorithm for the detector array. A detector system limited to one side of the object will almost certainly have lower efficiency than a detector system that could surround the object.

\subsection{Determining Enrichment}

The purpose of this section is twofold: 1) to discuss how enrichment was related to the point kinetics predicted first and second moments, and 2) to show how the point kinetics first and second moments vary with enrichment for uranium spheres of different sizes.

First, it is important to emphasize that the following section will not determine uranium enrichment directly from the point kinetics equations. Instead, factors that can be easily related to enrichment are found directly from point kinetics, and simple simulations that account for geometry effects then relate that value to a uranium enrichment level. The value within the point kinetics that is used to inform about uranium enrichment is the probability of induced fissions, $p$. This value can be 
directly related to $k_{e f f}$ and the system multiplication $M$ via the following equations:

$$
\begin{gathered}
k_{e f f}=\nu_{1}^{C} p \\
M=\frac{1}{1-\nu_{1}^{C} p}
\end{gathered}
$$

For bare uranium metal objects, the multiplication is a function of the enrichment, mass, and the geometry. Therefore it is very simple to simulate several enrichment levels of a given geometry to relate multiplication to enrichment for that given geometry. For the spheres of uranium previously investigated, the multiplication was related to the enrichment. This was used to get a relation between the neutron/photon moments and enrichment, which can be seen in Figure 3.15. Note that the values plotted are the moments of the particles leaving the object (Equation 3.36), and do not include detectors. The current method of modeling detection efficiency is so poor, that the values are not very meaningful.

From these figures, it is clear that neutrons generally have a steeper gradient than photons as enrichment is varied, especially as multiplication increases. For example, for the 4 and $6 \mathrm{~cm}$ spheres, the photon signatures have the lowest rates across all enrichments. This makes sense, because higher multiplication objects are inherently larger, leading to high levels of photon self absorption. For all signatures, the moments increase with enrichment. The second moments all increase more significantly than the first moments. With low enough uncertainty, any of these signatures could be used to predict enrichment. The effects of uncertainty on this system are explored in the next section.

Using point kinetics to predict enrichment in this way would still require some simulations to be run, requiring time and skill. The benefit is the simplicity of 

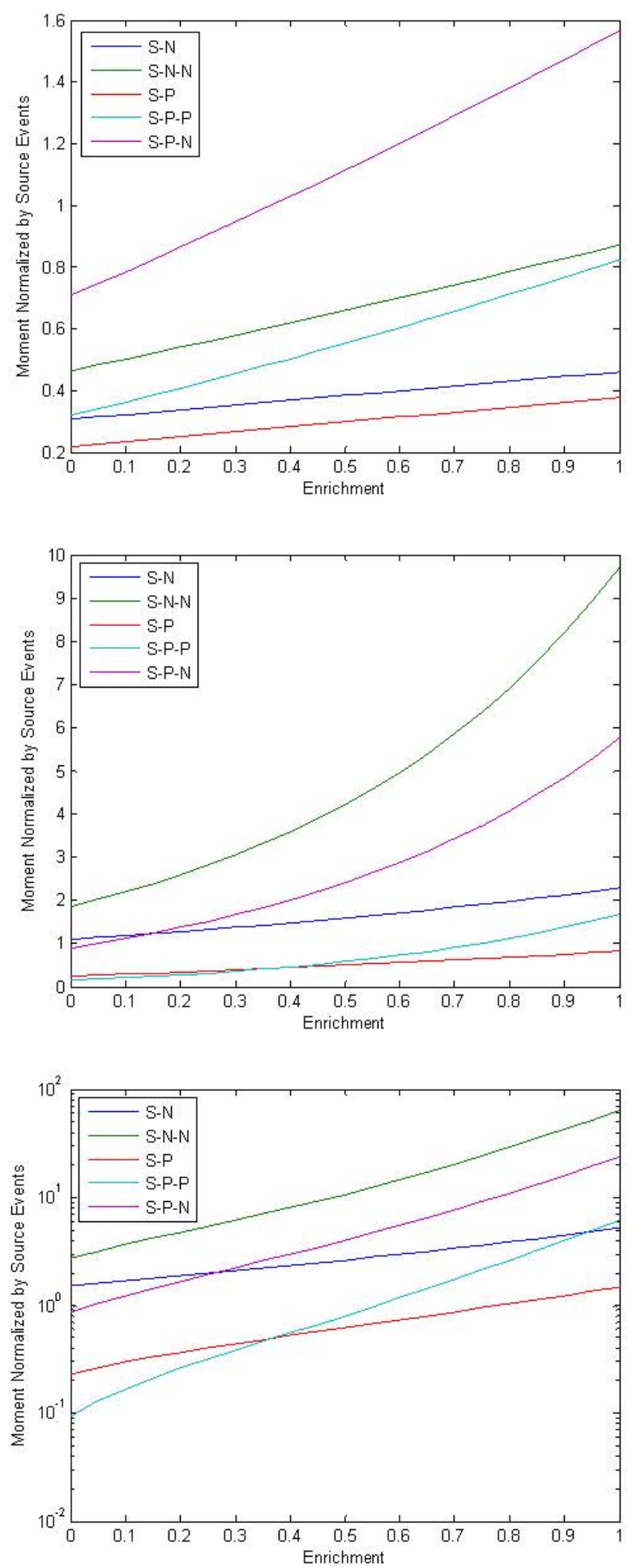

Figure 3.15: Moments vs enrichment for uranium spheres of 1 , 4, and $6 \mathrm{~cm}$ respectively. 
the point kinetics model, and the simplicity of the simulations which would need to be run to relate enrichment to multiplication. The previous chapter showed the problems of matching complex simulations to data. The uncertainties in the simulations of a complex detector system add up to give a significant difference between the measured and simulated results. However, these simulations are just of the inspection object.

\subsection{Effects of Input Values Uncertainty}

The results in the previous sections showed that adding interactions besides fission into the point kinetics equations increased accuracy significantly. Especially in the case of photon multiplets, a point kinetics framework that accounts for photon absorption and inelastic scattering interactions is necessary because realistic measurements will be heavily affected by these interactions.

The detriment of including these additional interactions is the added complexity and large number of input values. These input values each have uncertainty associated with them, and as these uncertainties propagate through the equations, they could become large enough to make the equations incapable of predicting enrichment.

To give a sense of how some reasonable uncertainties would affect the outcomes of these equations, an initial perturbation analysis was performed. A more rigorous perturbation analysis is left for future work. The input values were perturbed a given amount to determine the effects of these uncertainties on the first and second moment values. Care was taken to ensure that the values were perturbed in such a way that they didn't cancel each other out (e.g. if positively perturbing one value incurred positive change, and another positively perturbed value incurred negative 
change, the perturbations were assigned opposite values in order to add to the total system perturbation).

The perturbation values assigned are given in Table 3.3. The values chosen were deemed reasonable, but they are not based on any rigorous uncertainty analysis. The moments of the number distribution from the interactions, given by $\nu$, have a small uncertainty assigned to them. It is accepted by the nuclear science community that a large amount of nuclear data used in nuclear security applications need improvement (57). A 5\% range of uncertainties was assigned to all neutron and photon multiplicity moments, and a larger range of $10 \%$ was assigned to all cross section data to account for the additional uncertainties of using energy specific cross sections for a range of neutron energies. A reasonable uncertainty range of $\frac{1}{2} \mathrm{~cm}$ was assigned to the radius of the sphere, $R$, to account for uncertainties in measuring the geometric dimensions using radiography images.

The results of these perturbations are shown in Figure 3.16 for the first and second factorial moments of the neutron multiplet distributions. A couple of values are pointed out (values chosen are not significant) to show how the propagated uncertainty range in the moment values translates to an uncertainty range in the enrichment values. While the level of precision in the enrichment determination using these signatures is not overly impressive, it is still useful. In some applications, it is sufficient to differentiate DU from LEU from HEU, which the level of precision demonstrated here is clearly capable of. It is interesting that the second moment is less affected by the uncertainty propagation than the first moment. However, the lower enriched uranium is less sensitive to neutron multiplicity, so the higher precision on the left of the graph doesn't lead to significantly higher precision in estimating enrichment.

The photon moments predicted by these point kinetics equations are more com- 


\begin{tabular}{|ll|}
\hline$\nu_{1}^{C}$ and $\nu_{2}^{C}$ & $\pm 2.5 \%$ \\
$\nu_{1}^{D}$ and $\nu_{2}^{D}$ & $\pm 2.5 \%$ \\
$\nu_{1}^{E}$ and $\nu_{2}^{E}$ & $\pm 2.5 \%$ \\
$\nu_{1}^{F}$ and $\nu_{2}^{F}$ & $\pm 2.5 \%$ \\
$\nu_{1}^{M}$ and $\nu_{2}^{M}$ & $\pm 2.5 \%$ \\
$\nu_{1}^{R}$ and $\nu_{2}^{R}$ & $\pm 2.5 \%$ \\
$\sigma_{f} 14 \mathrm{MeV}$ & $\pm 5 \%$ \\
$\sigma_{(n, 2 n)} 14 \mathrm{MeV}$ & $\pm 5 \%$ \\
$\sigma_{(n, \gamma)} 14 \mathrm{MeV}$ & $\pm 5 \%$ \\
$\sigma_{(n, \gamma)}$ fission energy & $\pm 5 \%$ \\
$\mathrm{R}$ & $\pm 0.25 \mathrm{~cm}$ \\
\hline
\end{tabular}

Table 3.3: Table of perturbations
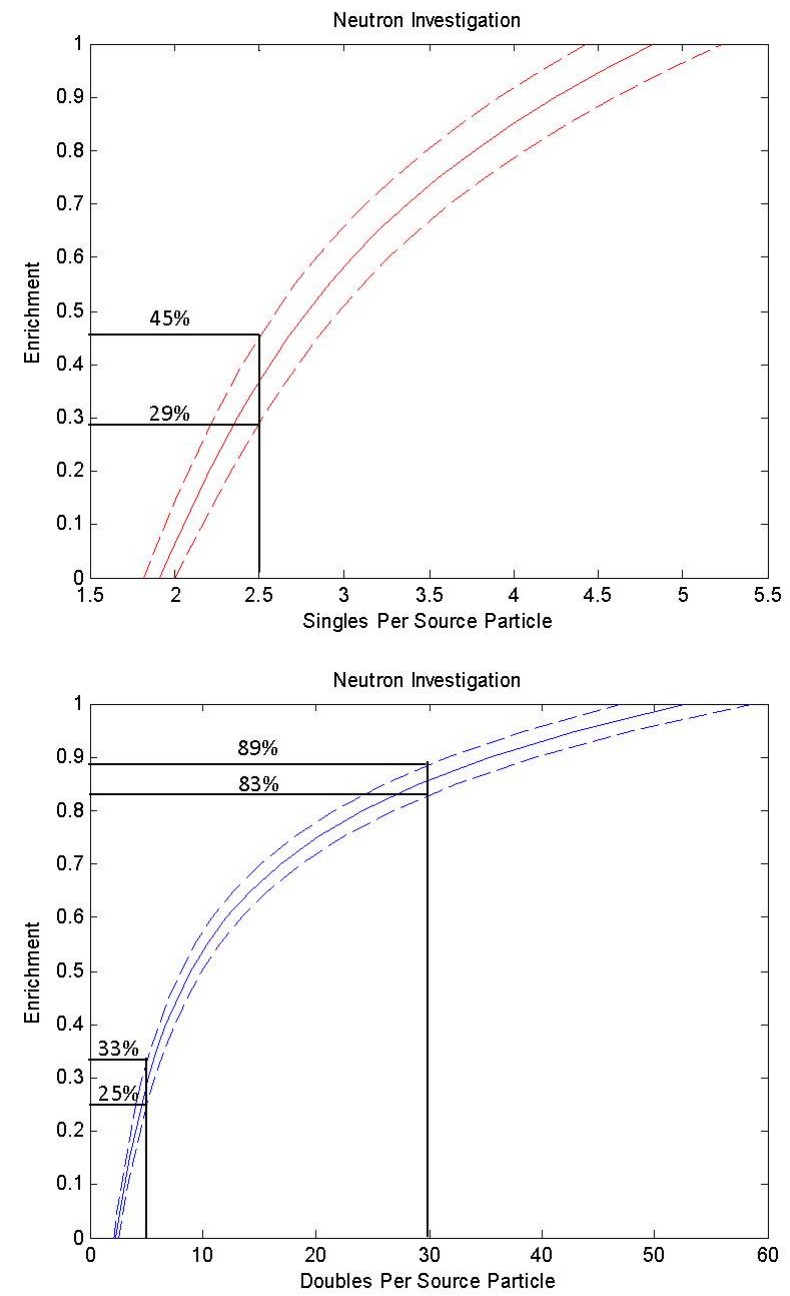

Figure 3.16: Propagated perturbations (seen in Table 3.3) for the first and second factorial moments of neutron multiplets escaping a $6 \mathrm{~cm}$ uranium sphere. 
plex than the neutron moments, and are reliant on many more nuclear data inputs. In particular, the uncertainty in the geometry leads to high levels of uncertainty, due to the high rates of self attenuation. Figure 3.17 demonstrates the effect of the geometric uncertainty propagated through the point kinetics predictions, without other uncertainties included.

Figure 3.18 shows the effect of all propagated uncertainty. The propagated uncertainty in the photon moments is clearly more significant than that of the neutron moments and a large percentage is geometric. However, it is useful to know that improving on the geometric precision has a large effect on improving the photon moment precision. Once again, the precision level is likely sufficient to differentiate DU from LEU from HEU. 

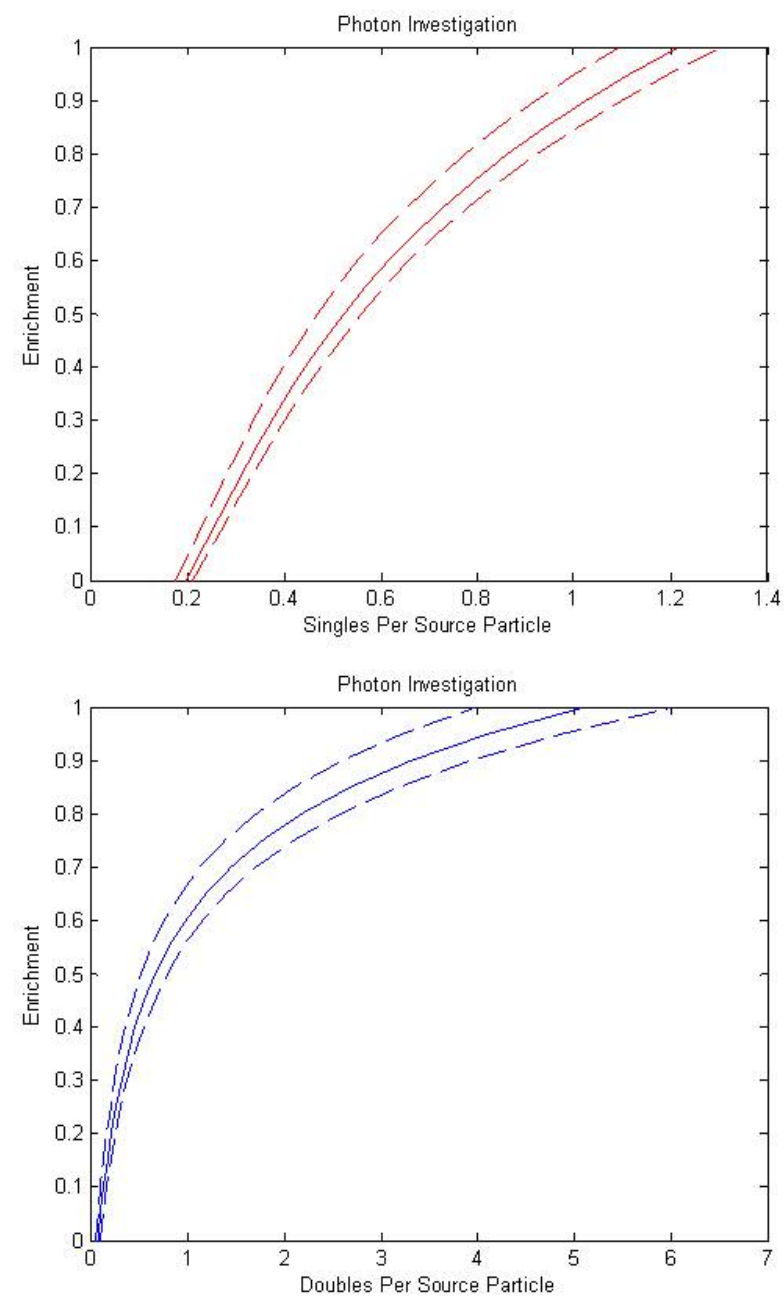

Figure 3.17: The effect of the radius, $R$, with $\pm 0.25 \mathrm{~cm}$ uncertainty propagated through to the first and second factorial moments of photon multiplets escaping a $6 \mathrm{~cm}$ uranium sphere. 

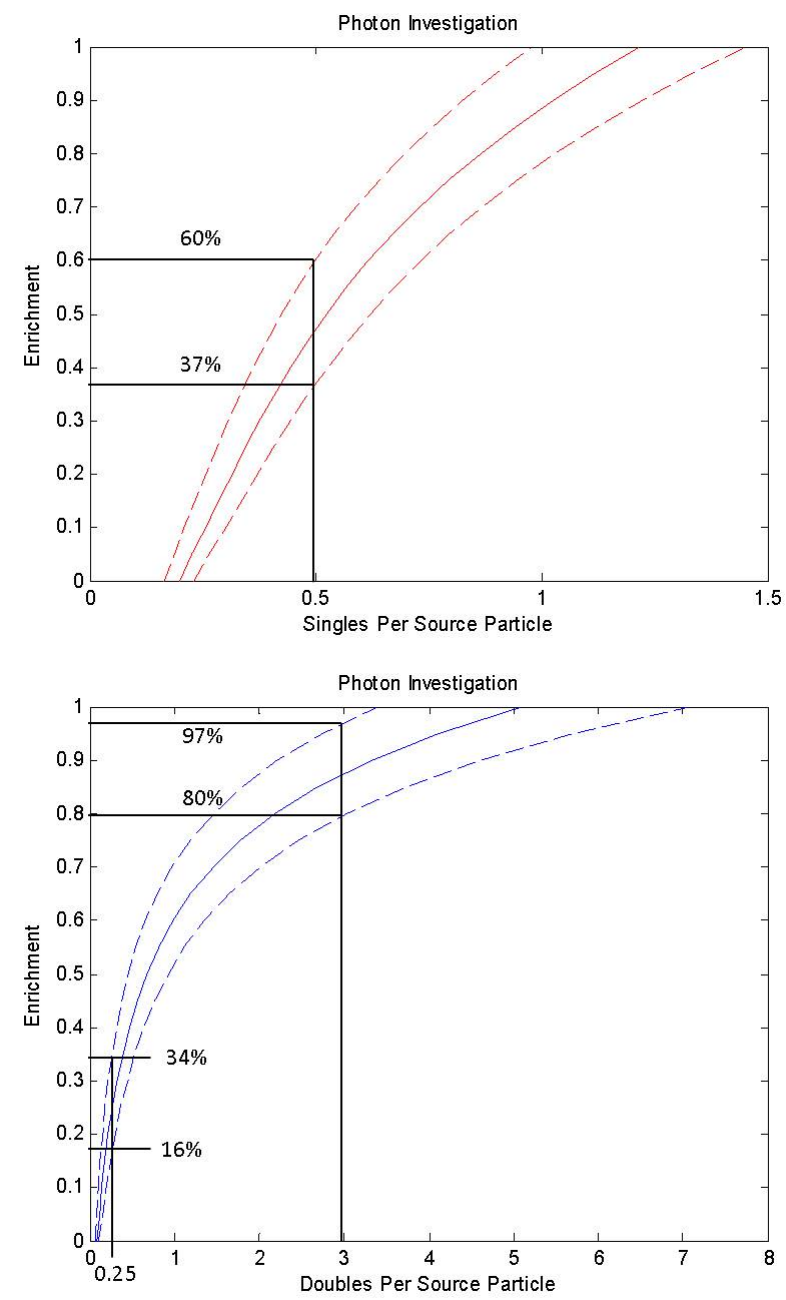

Figure 3.18: All propagated perturbations (seen in Table 3.3) for the first and second factorial moments of photon multiplets escaping a $6 \mathrm{~cm}$ uranium sphere. 


\subsection{Comparison to Experimental Data}

Since experimental data was taken of neutron and photon single and double source correlations, it is worthwhile to compare the point kinetics moments to these measured values. The efficiency of the detector array in the measurements was $4.7 \%$ and the total thickness that the source neutron travels through hollow cylindrical casting is $3.8 \mathrm{~cm}$. The DU casting is $0.3 \%$ enriched with a multiplication of 1.11. Table 3.4 shows the comparison between the point kinetics predicted and measured moments.

Previous sections showed that detector characteristics, like cross-talk and dead time, have significant effects in the total moments, and not modeling these detector aspects in the point kinetics led to poor results. Given that NMIS has even less detectors, which leads to larger effects from dead time, a close match is not expected. However, the DU casting has very low multiplication, which means the dead time effects will be less severe.

Given these cautious expectations, the comparison between the data and point kinetics shown in Table 3.4 is quite good, particularly for the neutron moments. It is especially interesting that the neutron second moment is actually predicted better by the point kinetics than by the complex MCNP PoliMi model investigated in the previous chapter.

Table 3.4: Comparison of point kinetics moments to measurements taken by NMIS

\begin{tabular}{|llll|}
\hline Moment & Point Kinetics & Measured Data & \% Difference \\
\hline Neutron First Moment & 0.0493 & 0.0432 & $-14 \%$ \\
Neutron Second Moment & 0.0039 & 0.0045 & $14 \%$ \\
Photon First Moment & 0.0118 & 0.0150 & $21 \%$ \\
Photon Second Moment & 0.0003 & 0.0010 & $69 \%$ \\
Photon-Neutron Moment & 0.0020 & 0.0025 & $20 \%$ \\
\hline
\end{tabular}


The photon moments do not match as closely, and this is not terribly surprising. The photon non-absorption probability used in the point kinetics model was determined by modeling a uranium sphere with a source uniformly distributed in the sphere. This geometry and source location are significantly different. Another factor is that the photon second moment in particular is a low-rate signature, leading to larger contributions from background and cross-talk.

The source-photon-neutron signature is again quite good, though not as well predicted here as in the previous section. However, given that cross-talk can have significant effects on comparing the point kinetics to real detector data, this signature's resilience against that makes it a very promising signature for comparison.

It would be interesting to see how well the point kinetics equations would model higher multiplication objects. It would also be interesting to see how the predictions compare to a detector system that is optimized to reduce dead time and cross time, meaning a system with many detectors and an algorithm that rejects adjacent detections to correct for cross talk. APNIS is a good example of such a system (2).

\subsection{Discussion}

The goal of this chapter was to build a point kinetic model that both maintains its simplicity, while also incorporating some of the complexities of a sizeable uranium object being interrogated by $14 \mathrm{MeV}$ neutrons. In doing so, another tool for

predicting enrichment using this source correlated first and second moments was investigated. Additionally, the higher order coincidences contributing to our measured moments were explored.

Because of the high energy of the source neutrons, the $(n, 2 n)$ source interaction has a significant effect and is worth modeling. The high energy of the source 
neutron also means the both ${ }^{235} \mathrm{U}$ and ${ }^{238} \mathrm{U}$ may have source induced fissions or source induced $(n, 2 n)$ interactions. The results of this study suggested that this point kinetics model accounted for this well, though it certainly adds complexity.

In order to realistically model the photon multiplets escaping a uranium geometry, it is necessary to model the self absorption; fission photons produced from 14 $\mathrm{MeV}$ induced fission for both ${ }^{235} \mathrm{U}$ and ${ }^{238} \mathrm{U}$ isotopes; fission photons produced from fission energy neutron induced fission for ${ }^{235} \mathrm{U}$; and inelastic scattering events for $14 \mathrm{Mev}$ and fission energy induced interactions for both ${ }^{235} \mathrm{U}$ and ${ }^{238} \mathrm{U}$ isotopes. This adds up to a large amount of input values. Given that the neutron input values also affect the photon multiplets, the total amount of input values affecting the photon distributions is significantly greater than that of neutrons, which tends to lead to higher uncertainty.

Overall the agreement of these simple point kinetic equations to the more complex 3D Monte Carlo models of the transport equation is quite impressive. The neutron and photon distributions escaping a variety of sizes and multiplications of uranium spheres was closely predicted by the point kinetic equations. It was especially interesting to see how resilient the photon distributions were to large amounts of polyethylene shielding, which significantly affected the neutron distributions. This strength could make the photon distributions useful despite their complex nature. The signatures leaving the inspection object were generally well modeled, and were clearly sensitive to the multiplicative properties of the object.

The current point kinetic equations did not model the detection of these escaping particles well for an NMIS type setup, and this issue was left for future work. As discussed, a detector can be optimized to minimize dead time and cross talk. Despite this issue, the point kinetics predictions of the measurements of the bare DU casting from the previous chapter was fairly good, especially for the neutron 
distributions. Improvement to this model could lead to better understanding of the photon and neutron moments detected by an API system, which could allow these signatures to be used for enrichment determinations.

While the previous chapter found the photon-only signatures to not be a significant improvement over the neutron signatures in any scenario, this chapter showed a scenario where photon-only signatures could potentially be of use. Modeling the higher order coincidences of the photon signatures escaping the polyethylene shielded uranium sphere was significantly more successful than the neutron signatures, due to the complex radiation transport involved in neutron moderation.

Future investigations of this work could include the following topics:

- Determining a method to calculate the photon non-absorption for a variety of geometries. This value has a large effect on the output of the point kinetics model. For this study, it was always determined empirically with MCNPx PoliMi simulations, which is a weakness

- Improving the method of modeling detection efficiency to include a correction for dead time effects.

- Comparing point kinetics predictions to results from a detector system that is optimized to reduce dead time and cross talk.

- Exploring the effects of radiography resolution. The utility of the photon signature relies heavily on the ability to get the geometry information from radiography in order to correct for self attenuation. Depending on the application, there may be time or neutron flux restraints that would allow for low image precision levels. If only low precision, high uncertainty geometry information is possible, the photon signature is likely not useful. It would be useful to 
explore this issue in more depth.

- Rebuilding this framework with a DD neutron source of $2 \mathrm{MeV}$ neutrons. Since $2 \mathrm{MeV}$ is within the fission neutron energy range, it may allow for the sourceenergy-neutron and fission-energy-neutron equations to be combined into one equation. It would also allow the $(n, 2 n)$ interaction to be dropped out of the source-energy neutron equation. This would significantly reduce the number of input values needed, which would greatly simplify the equations. 


\section{4 | Source Correlated Gamma-ray Spectroscopy}

In this chapter, the discussion shifts to a different type of signature than was discussed in the previous two chapters. This chapter will focus on the energy component of the source-photon peak by incorporating a gamma spectrometry detector. The gamma-rays are induced by the DT source neutrons, correlated in time and direction to the source neutron by the API method, and detected by the gamma spectrometry detector.

The goal is to investigate if any signatures related to enrichment appear in the photon energy spectrum for polyethylene shielded uranium, when the neutron signatures are potentially not usable. If the neutrons released from a fission chain would induce inelastic scattering gamma-rays that show up in the energy spectrum, that signature could potentially be used to predict enrichment. The interaction cross section is small for fission energy neutrons inelastic scattering in polyethylene so the noise has to be low enough to allow for reasonable signal to noise, which is why the API technique is employed.

In order to investigate this potential signature, it is first necessary to demonstrate the ability of this API prompt gamma spectrometry technique to identify materials in a narrow field-of-view, and have low interference from surrounding material. This is important because this technique relies on the ability to correlate the induced fission neutrons to the inelastic scattering gamma-rays they produce, which 
is a weak signature, so any gamma-rays from surrounding maerial would have a significant effect on the ability to separate out and predict the desired signature.

It is then necessary to observe the source correlated gamma-ray signature that is observable from shielded uranium. However, since the source neutrons also induce gamma-rays within the shielding material, it is necessary to separate out the signature induced from just the fission neutrons. Other related explorations of these signatures in support of this goal will also be included.

Just as each chapter has added to the generalized understanding of these API prompt signatures, this chapter unfolds the source-photon signature to look at the photon energies. While the neutron energy is evident from its time-of-flight, since the speed of a neutron is related to kinetic energy the induced gamma-rays travel at the speed of light regardless of energy. Knowing the energies of the photons can identify characteristic gamma-rays which will provide information about the materials present.

\subsection{Theory}

The physical mechanism behind this technique is to bombard a material with fast neutrons, which excite the nuclei via various interactions, and cause the release of gamma-rays with energies that are signature of the nuclear structure of the isotope. The detection of these characteristic gamma-rays can then be used for material identification. Different techniques utilize different types of neutron interactions, which dictates the neutron energy and the detection time-frame used. Since this research focuses on the API technique with $14 \mathrm{MeV}$ neutrons, only gamma-rays that reach the detector within a small time window of the source particle detection (a few hundred nanoseconds at most) will be included in the final gamma-ray energy 
spectrum, which primarily limits the relevant interactions to inelastic scattering.

\subsubsection{Detector Choice}

Due to the use of high energy neutrons, there are limitations in gamma-ray spectrometry detectors based on neutron damage and activation of detector materials. Due to the small correlation window, a short detector decay time is preferable, because it allows for better correlation time resolution. A high purity germanium (HPGe) detector has the advantage of good energy resolution and does not activate under neutron irradiation in a way that interferes with measurements. However, the germanium crystals are sensitive to damage from high energy neutrons, which eventually degrade the detector energy resolution. This issue is discussed in greater detail in Appendix D. Although n-type HPGe detectors are less prone to these problems, the longer charge collection time and lower efficiency due to small crystal size are also drawbacks for this application.

A lanthanum bromide $\left(\mathrm{LaBr}_{3}(\mathrm{Ce})\right)$ detector would be a good option due to its good energy resolution, very short decay time, and high light yield. However, it has significant activation under $14 \mathrm{MeV}$ irradiation, with a spectral continuum all the way up to around $3 \mathrm{MeV}$ (58). This would cause many useful characteristic gammarays in the $1-3 \mathrm{MeV}$ energy range to compete with the high background internally in the detector. This could be a significant disadvantage, depending on the isotopes that are being identified for the application.

A sodium iodide (NaI) is a good option when low resolution is acceptable. It has a short decay time of around $250 \mathrm{~ns}$ and good light yield (58). The NaI is not sensitive to damage, but the PMT does shift due to the high flux environment (discussed in Appendix E). The continuum is lower than the $\mathrm{LaBr}_{3}$, ending around 
$2 \mathrm{MeV}$.

The nature of the technique requires gamma-rays from interactions with high cross sections and/or high energy characteristic gamma-rays, since the interaction probability and detector efficiency combined with high background leads to low detection rates of relevant gamma-rays. These qualifications limit the materials that can be identified using this technique. A good resource for gamma-ray energies and cross sections induced by $14 \mathrm{MeV}$ neutrons for various isotopes can be found in the International Nuclear Data Committee (INDC) report (59).

\subsection{Relevant Literature}

The use of a DT neutron generator for active interrogation followed by prompt gamma-ray spectroscopy to identify unknown materials for security reasons has been done by quite a few groups, usually for non-destructively identifying materials in explosives $(19 ; 20 ; 26)$. In many instances the gamma-rays are not correlated to a source, but just collected over the whole measurement time $(58 ; 1)$. Other groups utilize the timing characteristics of the source to inform the gamma-ray energy spectrum. For example, the Pulsed Fast/Thermal Neutron Analysis technique uses a pulsed generator to collect gamma rays from inelastic scattering when the neutron pulse is on, and prompt gamma rays from absorption of neutrons when the pulses is off (58).

The three main systems in open literature that use an API DT generator to generate source correlated prompt gamma-ray spectra for material identification purposes are EURITRACK (60), UNCOSS (61), and SENNA (62). All three systems were built for non destructive explosives detection.

The EURITRACK system consists of a portable DT generator which includes an 
8x8 marix of YAP:Ce alpha detectors that are coupled to a multi-anode PMT. Some large volume $\mathrm{NaI}(\mathrm{Tl})$ detectors are arranged inside a structure similar to a fixed portal monitor, as well as a 5" $\times 5$ " BC501A liquid scintillation detector in order to monitor the attenuation of the neutrons. Using the pixelated alpha detector to specify direction, and the time-of-flight to determine depth, the system is able to focus on specific areas within the large cargo containers to inspect. (60)

The analysis of data taken by EURITRACK includes correcting for gamma and neutron attenuation in the container in order to get accurate chemical ratios. The chemical ratios of carbon, oxygen, and nitrogen are used to determine if the material is contraband or benign. (63)

In more recent years, the intended use of the EURITRACK concept has been expanded to look at applications such as spent fuel characterization and shielded SNM detection. They also look at the option of using this technique to identify shielding material in SNM configurations. (20)

The UNCOSS system was developed by many of the same people involved with EURITRACK, but the focus for this system is underwater explosives detection (61). The system was fit into a small remotely operated vehicle, and consisted of an ING27 neutron generator with nine alpha pixels and a $3 \times 3 \mathrm{in}^{2} \mathrm{LaBr}_{3}(\mathrm{Ce})$ detector. The analysis method used is the same as for EURITRACK.

The SENNA system was independently developed in Russia and is similar to the EURITRACK system (62). The system consists of ING-27 neutron generator with a $3 \times 3$ matrix of $1 \mathrm{~cm}^{2}$ alpha detectors, along with two $6.3 \times 6.3 \mathrm{~cm}^{2} \mathrm{BGO}$ gamma ray detectors. SENNA was developed to inspect boxes and suitcases rather than cargo containers, but appears to use a similar analysis approach to EURITRACK.

With regards to FNMIS, gamma-ray spectrometry was already planned to be incorporated into future models, and this work partially supports that goal. As 
mentioned previously, simulations using shielding identification for shielded uranium assemblies with NMIS have been performed (1). In this study, the gamma rays were not correlated in time to the source event, and it was noted that other materials present in the setup were seen in the spectra, such as aluminum from the detector stand and steel from the turntable. Experimentally, NMIS has been shown to be able to identify some select bulk materials using source correlated prompt gamma-ray spectrometry on an analog system with a $1 \mu$ s correlation time window and no uranium present (64). The work here expands this previous work by using a digital correlation system and smaller time windows; looking at combinations of materials; and looking for signatures related to enrichment instead of material identification.

\subsubsection{Experimental Setup}

The API DT neutron generator used for these measurements is a VNIIA ING27 generator with a continuous flux rated to go up to $10^{8}$ neutrons/second. For dosimetry reasons, the generator was run at a maximum of around $1 \times 10^{7}$ neutrons/second. This model of neutron generator has a built in segmented semiconductor alpha detector. The segmented detector is a line of 15 segments, each $30 \times 30 \mathrm{~mm}^{2}$ in size. (65)

The HPGe detector used was an Ortec Gamma-X N-type coaxial "pop-top" model detector cooled by liquid nitrogen. The signal from the detector anode was run directly into a CAEN 57244 channel 14 bit, $100 \mathrm{MS} / \mathrm{s}$ digitizer, and analyzed by the CAEN pulse height analysis control software (DPP-PHA).

The signals from alpha pixels 6,7 , and 8 were input into three of the digitizer channels, along with the HPGe signal. Target objects were placed about $55 \mathrm{~cm}$ away 


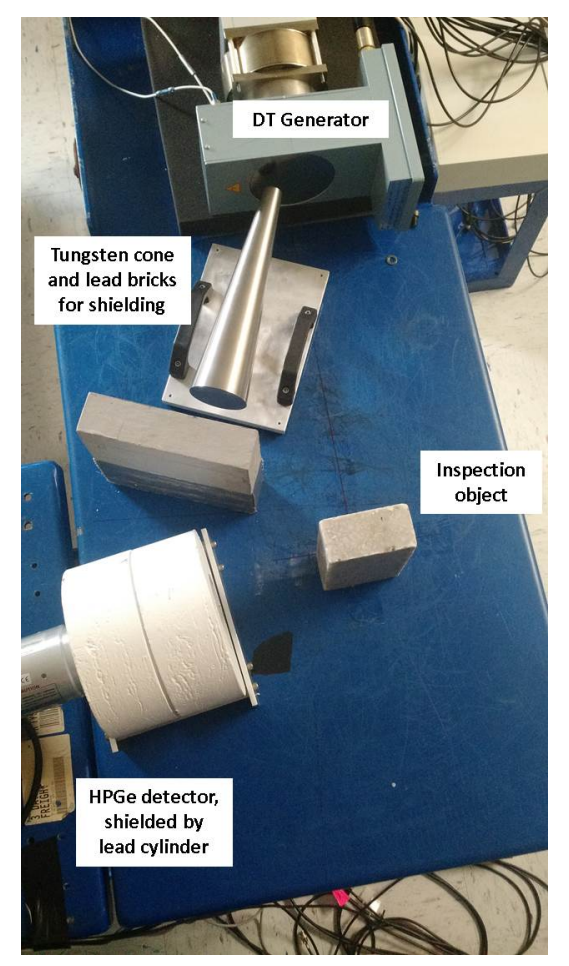

Figure 4.1: Setup of geometry with HPGe detector included.

from the front of the generator, with the detector placed to the side. The HPGe detector had a tungsten cone $30.5 \mathrm{~cm}$ long and lead bricks between the generator and the Ge crystal to shield the crystal from the source neutrons. There was also a lead shield that surrounded the Ge crystal for additional shielding. With this level of shielding, it was determined that the detector damage from the DT generator would be insignificant, which is discussed further in Appendix D. The setup can be seen in Figure 4.1.

A previous measurement had determined the field of view of the pixels of the alpha detector. The three pixels used for these measurements span about a 20 degree field of view, as can be seen in Figure 4.2. All targets were placed within this field of view.

The DPP-PHA CAEN software converts the pulses into triangular wave forms 


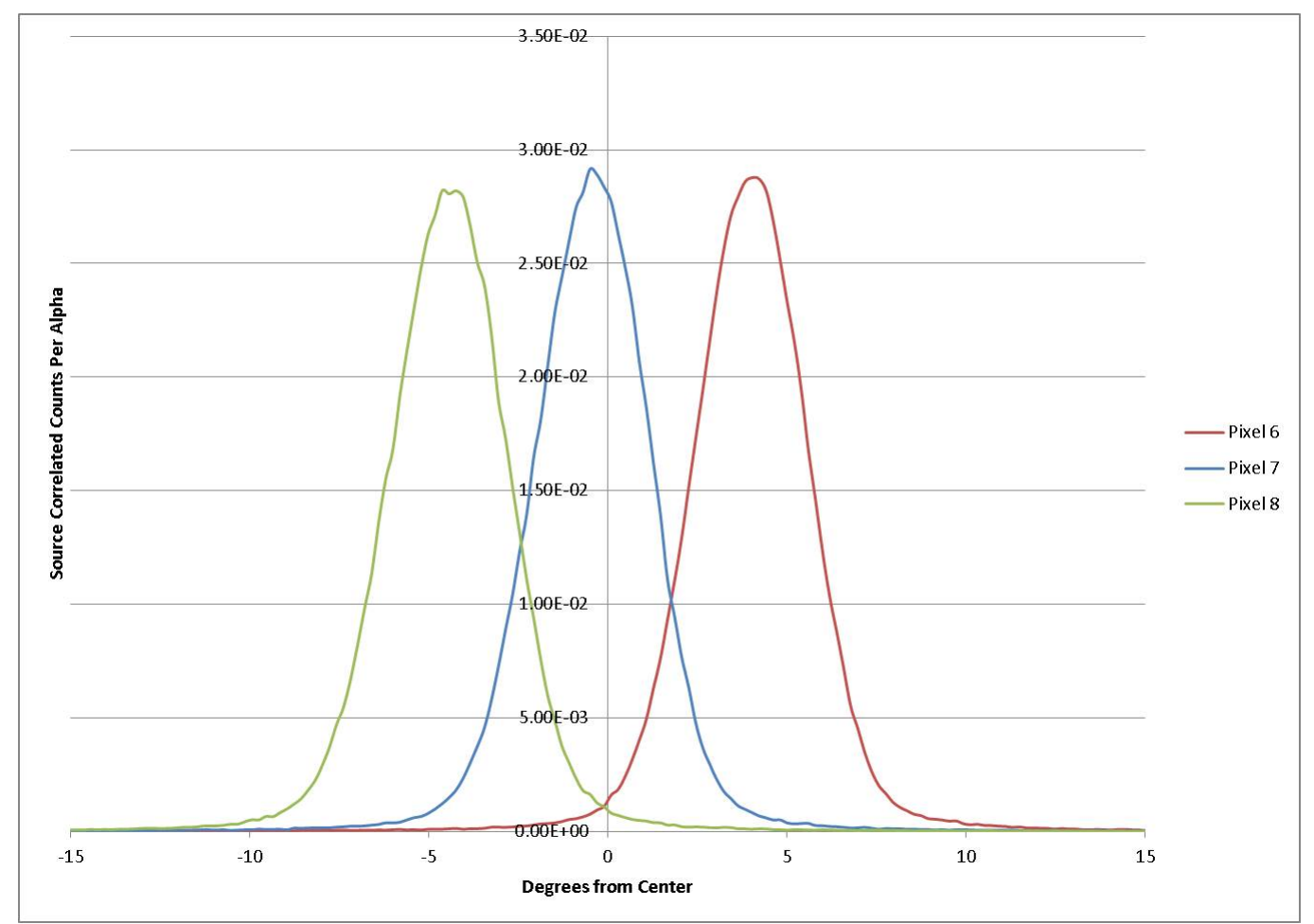

Figure 4.2: The angular field of view of each pixel with respect to the source spot.

based on user inputs. The pulse height of the triangular waveform is proportional to the energy deposited in the detectors and is recorded for spectrometry. The data was output in list mode, which included all timing and pulse height information for events that exceeded a user defined threshold. A post processing program* determined the correlations between the alpha pixel source pulses and the HPGe induced radiation pulses based on the timing information. The user can define a correlation time window as region of the true correlated induced radiation. The pulse height information from all pulses that fall into this correlation window are extracted and plotted into an energy histogram.

In order to determine an appropriate correlation window, a correlation plot is created to show the number of counts that occur in a given period of time following

*written by Seth McConchie 


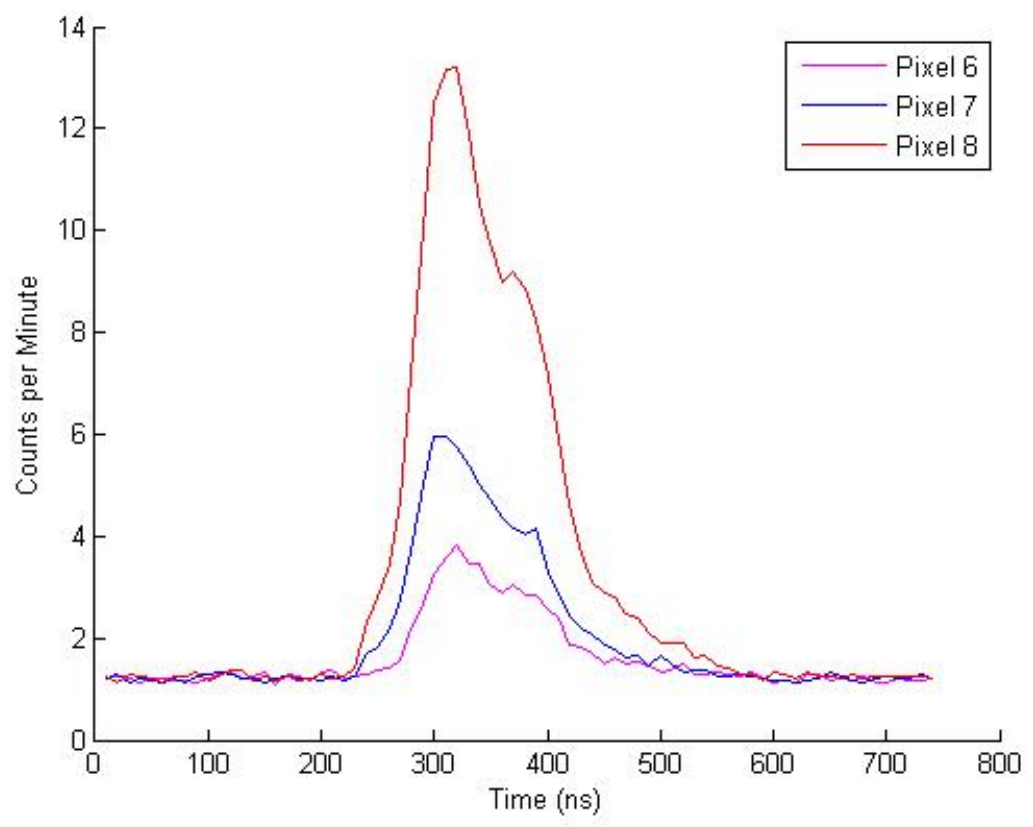

Figure 4.3: Correlation of the induced radiation detected in the HPGe detector following an detection in the specified alpha pixel.

the source event, such that time zero is marked by the detection of the alpha particle from the DT interaction. Figure 4.3 shows the peak from the induced radiation following the source event for each alpha pixel. The absolute difference in time between the source event and this peak does not represent the time of flight in this case, because it includes differences in cable lengths and pulse processing and detector properties. The pulse magnitude decreases from one pixel to the next due primarily to self attenuation in the object, such that gamma-rays induced in the object that are correlated to the pixel 6 need to travel through much of the object to reach the detector.

The true signature is estimated to be from 220 - 590 nanoseconds based on the plot in Figure 4.3. The pulse height information for the alpha pulses that fall in the correlation window for all three alpha pixels are plotted in Figure 4.4 with the red line. This pulse height spectrum is not useful for the analysis because the 


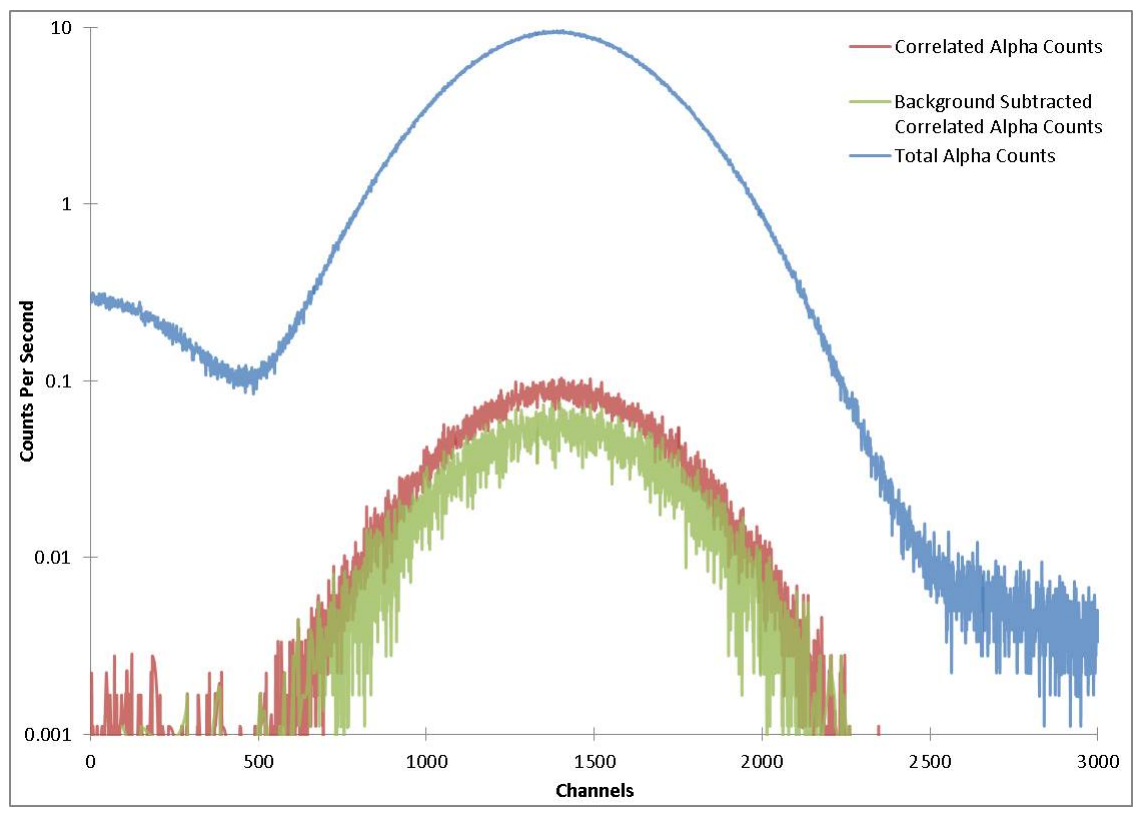

Figure 4.4: Pulse height spectra of the total alpha counts, alpha counts correlated to the HPGe detector, and correlated alpha counts with background subtracted.

alpha pulses serve only as a trigger, but it shown here for illustrative purposes. The highest blue line shows the pulse height distributions of all the alpha pulses for the uncorrelated pixels recorded during this 30 minute measurement. The main peak corresponds to true alpha pulses (around channels 475 up to 2500), while the lower energy tail corresponds to electronic noise and x-rays that are not correlated to a source event.

When looking at Figure 4.4, it is immediately obvious that the total number of correlated alpha pulses is a small percentage of the total number of alpha pulses. The likelihood that a source $14 \mathrm{MeV}$ neutron creates prompt gamma-rays in the target object, and that this prompt radiation makes it into the small detector crystal and then deposits enough energy to exceed the threshold is very low. Therefore this severe reduction in alpha counts is understandable.

Notice in the red line in Figure 4.4 there are still some low pulse height counts in the correlation plot that are likely not true alpha pulses. This implies that there 
is a small rate of accidental correlations. This is not very surprising when looking back at Figure 4.3, and noticing the significant background rate of around 1.5 counts/min per nanosecond after the source event. In order to subtract out this background contribution, a correlation window of the same size is taken in a background region of the correlation plot (starting at $1 \mu \mathrm{s}$ ), and the pulse height spectrum from this region is subtracted from the correlated pulse height spectrum. The results of this process are shown in Figure 4.4 by the purple plot. In this background subtracted spectrum, it appears that the accidental correlation rate is essentially non-existent, meaning that the spectrum will be made up of true correlated prompt radiation, though much of it is still true correlated background. It is clear that this method is an excellent way to reduce background, in spite of the significantly reduced count rate.

\subsection{Identification and Localization of Material}

The first goal was to verify that this setup could be used to identify characteristic gamma-rays above background and in a specified region without significant contributions from adjacent regions.

A different material was put into the field of view of each pixel side by side to see how well this technique could be used to identify and differentiate these objects. As shown in Figure 4.5, an aluminum annular cylinder was placed in the view of pixel 8, a graphite brick was placed in front of pixel 7, and a container of water was in view of pixel 6. For all measurements, the DT neutron generator continuously outputs between $9 \times 10^{6}$ and $1 \times 10^{7}$ neutrons per second. A measurement was taken for 60 minutes. Table 4.1 gives the characteristic gamma-rays of some materials present in this section. 


\begin{tabular}{|c|c|}
\hline Energy (keV) & Element \\
\hline 803 & $\mathrm{~Pb}$ \\
834 & $\mathrm{Ge}$ \\
844 & $\mathrm{Al}$ \\
847 & $\mathrm{Fe}$ \\
931 & $\mathrm{Fe}$ \\
1014 & $\mathrm{Al}$ \\
1095 & $\mathrm{~Pb}$ \\
1238 & $\mathrm{Fe}$ \\
1590 & $\mathrm{~Pb}$ \\
1720 & $\mathrm{Al}$ \\
1770 & $\mathrm{~Pb}$ \\
1809 & $\mathrm{Al}$ \\
2211 & $\mathrm{Al}$ \\
2615 & $\mathrm{~Pb}$ \\
3004 & $\mathrm{Al}$ \\
4439 & $\mathrm{C}$ \\
6130 & $\mathrm{O}$ \\
\hline
\end{tabular}

Table 4.1: Energies of some of the prompt gamma-rays that are likely to be seen in spectra from these measurements

The results of the measurement are shown in Figure 4.6. In the total spectrum, shown in black, it is clear that a variety of materials are present; contributions from all three target objects are clear. In the total HPGe spectrum, evidence of the lead shielding (2.62 MeV) and iron in structural materials (1.24 MeV and 0.85 MeV) are evident, even though those materials are not prevalent in the target object. Aluminum is present, but it could be from either the table or the inspection object (3.00 $\mathrm{MeV}$ ). The main advantage of the correlated spectrum is that it is a narrow field of view of the alpha pixels, which clarifies what elements are present specifically in the target object.

By correlating the HPGe detector to each alpha pixel, it can be determined clearly what material is in the field of view. In green, the spectrum correlated to pixel 8 is shown, which was aimed at the aluminum target. The aluminum peaks 


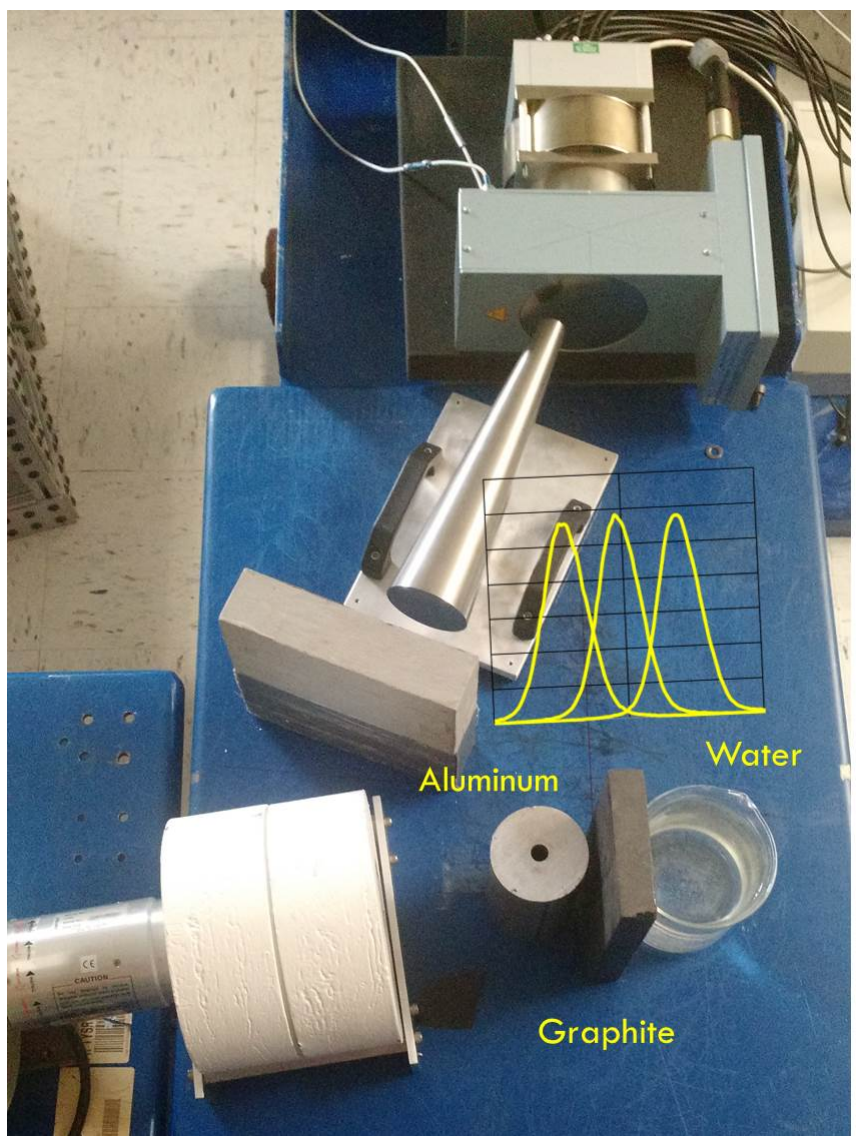

Figure 4.5: Experimental setup of measurement done to determine the ability of this technique to differentiate nearby materials.

are easy to identify, and are the strongest signatures (besides the large $511 \mathrm{keV}$ peak). The blue specrum is correlated to pixel 7 which was aimed at graphite. The resolution of the carbon peak ( $4.44 \mathrm{MeV})$ is noticeably worse than other peaks ${ }^{\dagger}$, making the peak not as clear, but definitively above background, along with the first escape peak (3.93 MeV). The red spectrum correlated to pixel 6, which was aimed at the container of water, is the most difficult to see. This is due both to the attenuation from the other two objects and the low efficiency of HPGe, especially

\footnotetext{
${ }^{\dagger}$ It is important to note that the poor resolution of the carbon peak compared to the rest of the peaks present is due to a physical phenomenon particular to the carbon nuclear interaction; when the carbon nucleus is excited, the decay time is so short that the nucleus is still recovering from the collision, which leads to Doppler broadening of the energy peak.
} 
for high energy photons. Despite this, it is still possible to identify the $6.13 \mathrm{MeV}$ gamma-ray peak from oxygen, as well as its first escape peak at $5.62 \mathrm{MeV}$.

It is very interesting how well the nearby objects are filtered out of these pixel correlated spectra. Although the objects are right next to each other, and the pixel views overlap slightly, it is very clear what the predominant material associated with each pixel is. The contribution from the adjacent materials in the spectra is hardly even discernible. The downside of this technique, again, is the very low count rates in the relevant source correlated energy peaks. The best way to combat this problem would be to add additional HPGe detectors to the setup. However, this increases the overall cost and weight (due to large amounts of shielding) to the setup.

A summary of these three measurements is shown in Table 4.2. In this table, the integrals of the peaks of interest of each measurement are given for both the correlated and total spectra, along with the uncertainty information, in units of counts per minute (CPM). Spectral background was subtracted from each integral, and included in the uncertainty determination. The peak to background (BG) ratio is also given for each gamma-ray energy peak, which is the counts in the highest count channel divided by the average of the background on either side of the peak. Using the count rates and background rates for each gamma-ray peak, the amount of time required for the counts to exceed the detection limits, as defined by L. A. Currie (66), was determined.

The results in Table 4.2 highlight the strengths and weaknesses of utilizing the peaks from the source correlated spectra versus those in the total spectra. The peak to background ratios are significantly higher for the source correlated spectra, which makes sense considering how effective this method is at reducing background. However, the count rates are so greatly reduced in the source correlation 


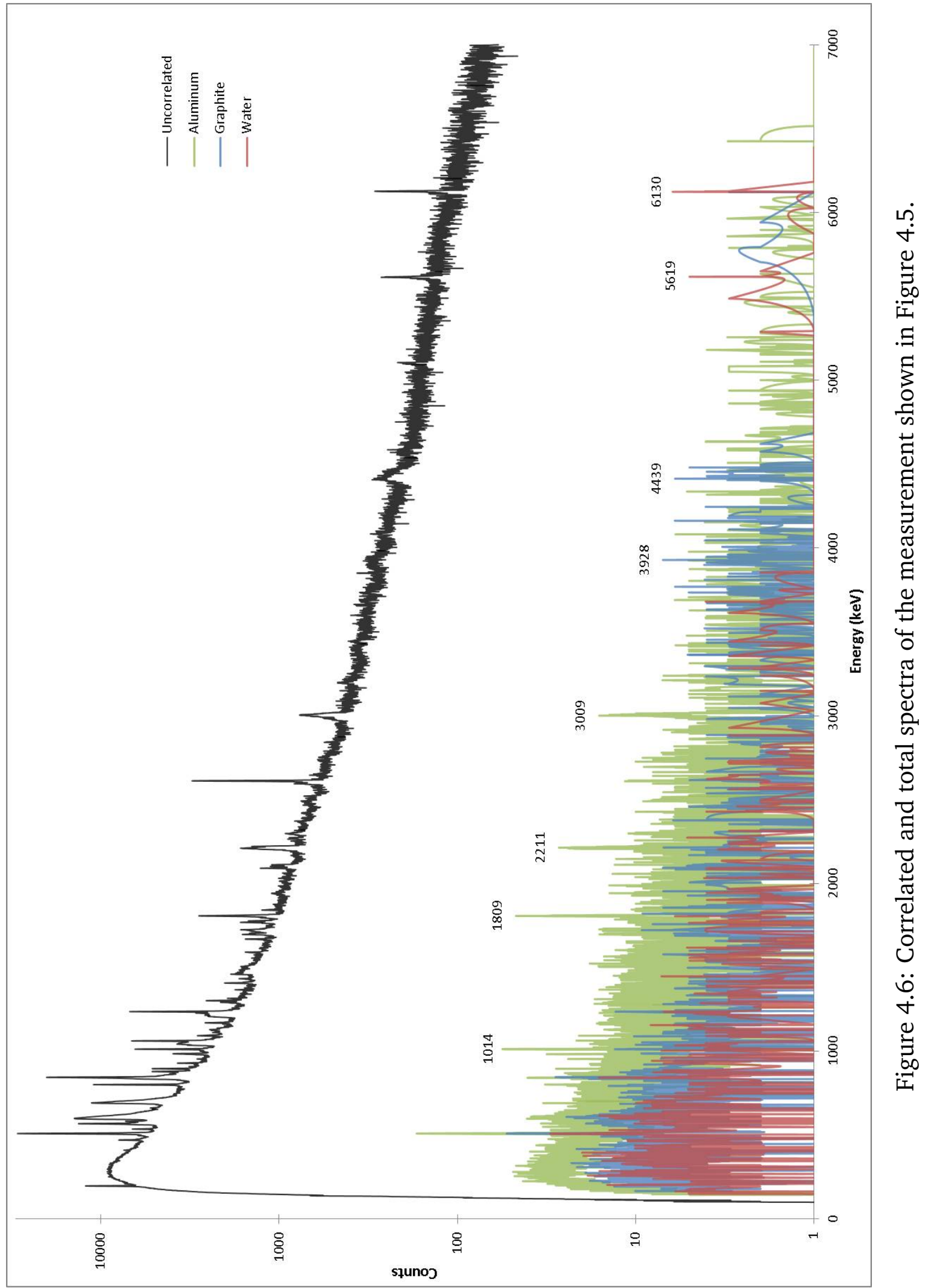


technique that the time to detectability is always significantly shorter for the peak in the total spectrum. However many of the peaks in the source correlation method are still detectable in less than 10 minutes. The oxygen peaks in water were the most difficult of those studied. However, it is important to remember that the field of view was small, and the attenuation high for that measurement.

Again, the main benefit of the source correlation method is not quantifiable in Table 4.2, which is that the non-relevant peaks drop out of the spectra, making it easier to determine what isotopes are present in the target object. The main objective of this study was to determine the ability of this technique to isolate specific materials. This ability has been clearly demonstrated.

Table 4.2: Integrals of the peaks of interest from spectra shown in Figure 4.6

\begin{tabular}{|c|c|c|c||c|c|c|}
\hline \multirow{2}{*}{$\begin{array}{c}\text { Energy } \\
\text { (MeV) }\end{array}$} & CPM & $\begin{array}{c}\text { Peak to } \\
\text { BG Ratio }\end{array}$ & $\begin{array}{c}\text { Time Until } \\
\text { Detectable } \\
\text { (min) }\end{array}$ & CPM & $\begin{array}{c}\text { Peak to } \\
\text { BG Ratio }\end{array}$ & $\begin{array}{c}\text { Time Until } \\
\text { Detectable } \\
\text { (min) }\end{array}$ \\
\cline { 2 - 6 } & \multicolumn{7}{|c|}{ Source Correlated Spectra } & \multicolumn{3}{c|}{ Total Spectra } \\
\hline \multicolumn{7}{|c|}{ Pixel 8 - Aluminum } \\
\hline 1.81 & $4.4 \pm 0.4$ & 9.00 & 3.7 & $233.6 \pm 4.4$ & 2.65 & 0.2 \\
\hline 2.21 & $4.2 \pm 0.4$ & 6.44 & 3.3 & NA - Hydrogen interference \\
\hline 3.00 & $2.9 \pm 0.3$ & 8.06 & 5.7 & $107 / 8 \pm 3.7$ & 1.69 & 0.7 \\
\hline \multicolumn{7}{|c|}{ Pixel 7 - Graphite } \\
\hline 3.93 & $1.1 \pm 0.3$ & 6.48 & 48.0 & $34.1 \pm 4.7$ & 1.24 & 12.3 \\
\hline 4.44 & $2.6 \pm 0.3$ & 12.18 & 5.6 & $83.5 \pm 4.2$ & 1.51 & 1.6 \\
\hline \multicolumn{7}{|c|}{ Pixel 6 - Water } \\
\hline 5.61 & $0.3 \pm 0.1$ & 12.86 & 73.1 & $20.6 \pm 1.5$ & 1.97 & 3.0 \\
\hline 6.13 & $0.5 \pm 0.1$ & 19.64 & 22.4 & $23.1 \pm 1.4$ & 2.59 & 2.1 \\
\hline
\end{tabular}

\subsection{Investigating Signatures from Shielded Uranium}

Now that the ability of this technique to locate and identify materials has been shown, the signature from the uranium shielded by polyethylene is investigated. 


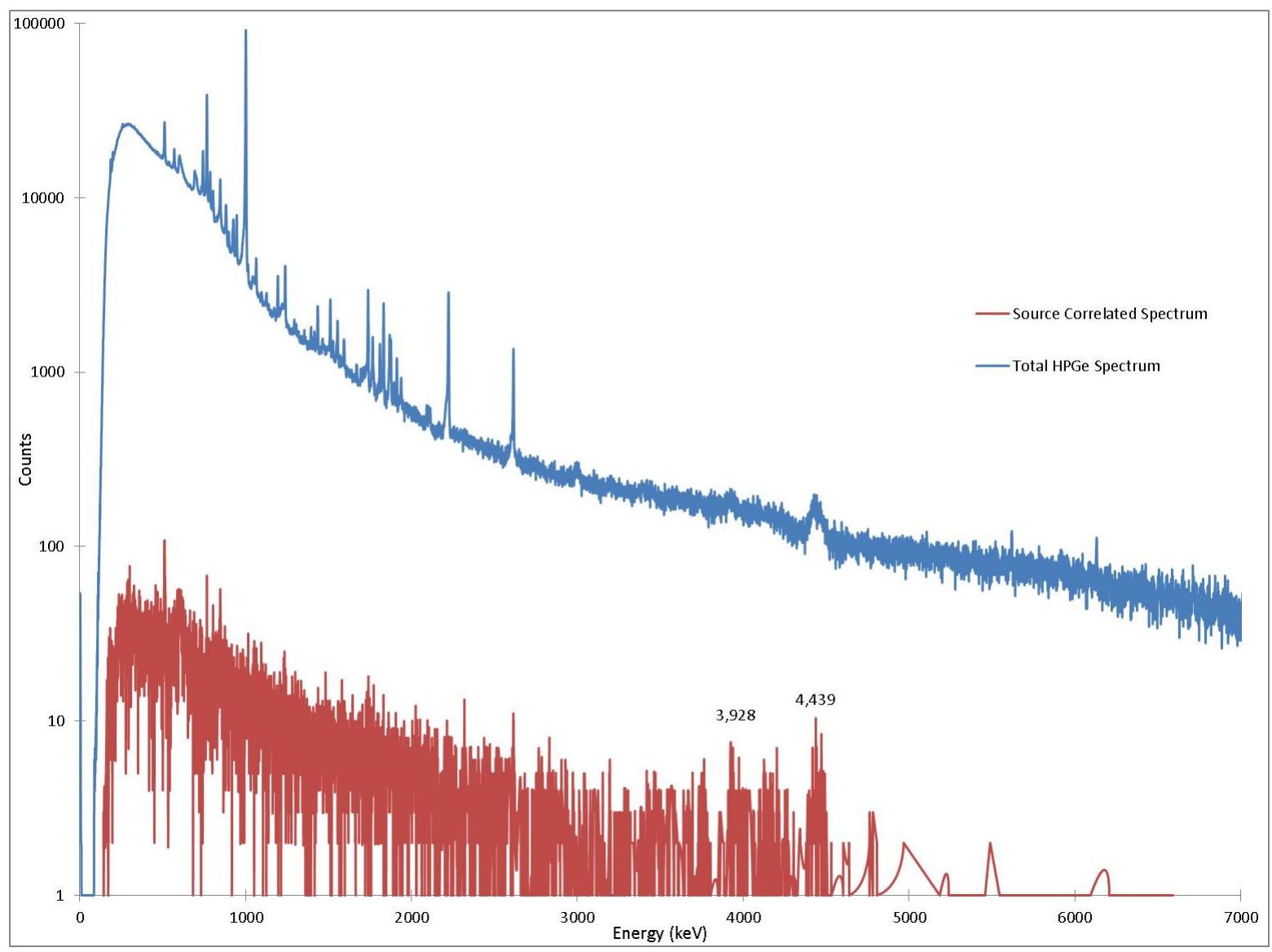

Figure 4.7: Correlated and total spectra of polyethylene shielded DU

The measurement of the DU casting surrounded by $5 \mathrm{~cm}$ of polyethylene was taken for 30 minutes (the DU casting surrounded by polyethylene can be seen in a later section in Figure 4.11). The results plotted in Figure 4.7 include the total spectrum taken by the HPGe detector during the 30 minute measurement, as well as the source correlated HPGe spectrum. Again, by excluding all data outside of the correlation window, the count rates drop dramatically. Table 4.1 can be used as a guide to identify many of the peaks present in the following spectra. For these particular spectra, the main carbon peak (4.4 MeV) and its escape peaks will be the signatures that identify the presence of the polyethylene shielding material. The hydrogen present in the polyethylene does not have a prompt activation signature. 
The strong presence of the $2.22 \mathrm{MeV}$ peak in the total spectrum is due primarily to thermalized neutron interactions with hydrogen, but these gamma-rays are not present in the prompt time region. However, the $4.44 \mathrm{MeV}$ peak from carbon and its first escape peak at $3.93 \mathrm{MeV}$ are present in both the total and correlated spectra in Figure 4.7.

Again, various elements are evident in the total spectrum, but the most prevalent signature of the correlated spectrum is the carbon peak from the polyethylene in the target object. The carbon is clearly evident despite the combination of materials, and is a fairly strong signature.

The next measurement was done with the DU casting and $5 \mathrm{~cm}$ of aluminum shielding surrounding it. In Chapter 2, aluminum shielding significantly affected the neutron and the photon signatures from uranium. However, the photon signature was affected because of strong inelastic scattering gamma-rays, which are useful to this technique.

The results plotted in Figure 4.8 again show both the total spectrum from this measurement as well as the source correlated spectrum. The aluminum signatures that are strongly evident are labeled in the correlated spectrum. Again, the total spectrum is complex with various contributions from shielding and structural materials, but the correlated spectrum makes the primary presence of aluminum quite clear. 


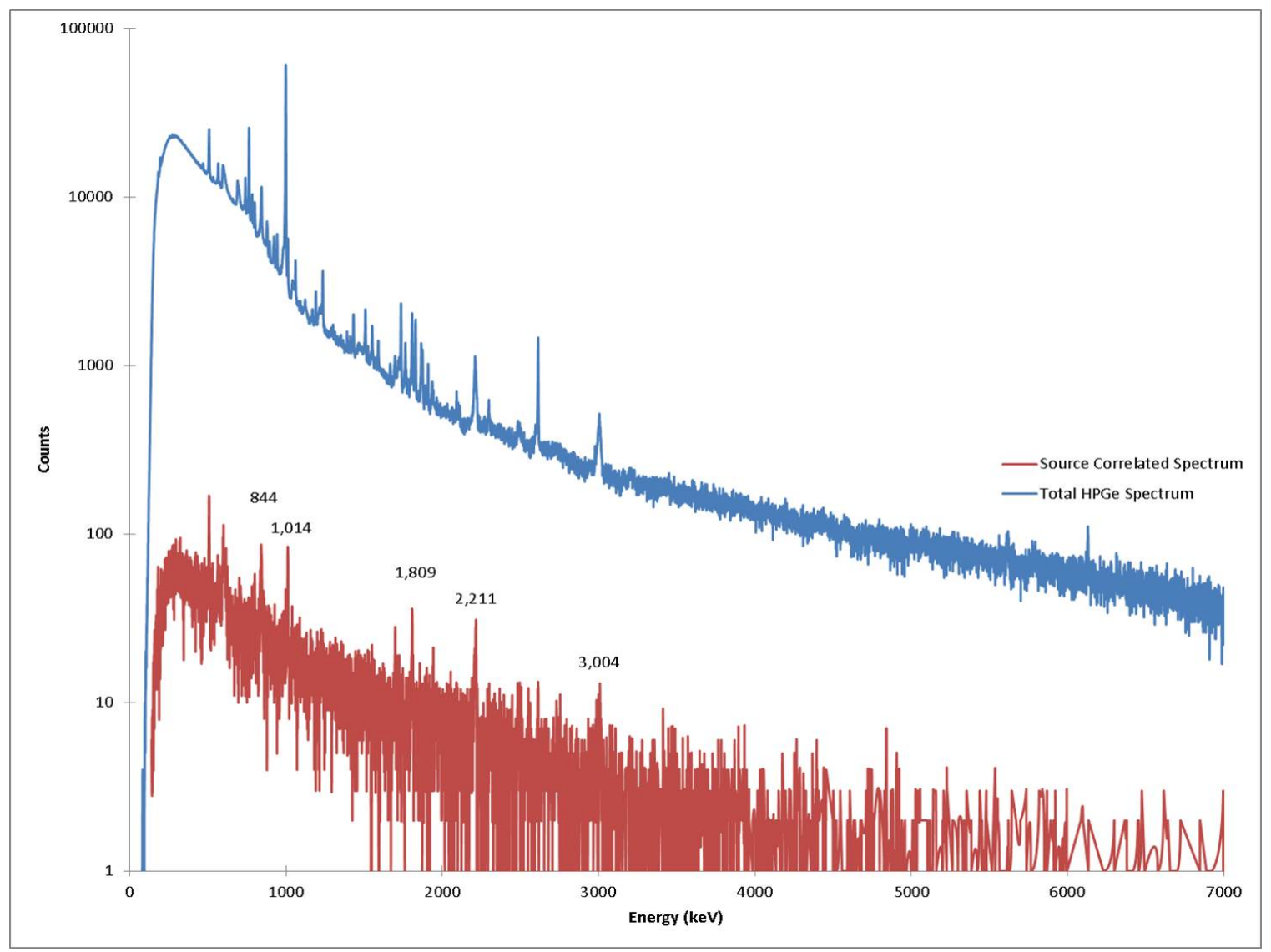

Figure 4.8: Correlated and total spectra of aluminum shielded DU.

Table 4.3: Integrals of the peaks of interest from spectra shown in Figures 4.7 - 4.8

\begin{tabular}{|c|c|c|c||c|c|c|}
\hline \multirow{2}{*}{$\begin{array}{c}\text { Energy } \\
\text { (MeV) }\end{array}$} & CPM & $\begin{array}{c}\text { Peak to } \\
\text { BG Ratio }\end{array}$ & $\begin{array}{c}\text { Time Until } \\
\text { Detectable } \\
\text { (min) }\end{array}$ & CPM & $\begin{array}{c}\text { Peak to } \\
\text { BG Ratio }\end{array}$ & $\begin{array}{c}\text { Time Until } \\
\text { Detectable } \\
\text { (min) }\end{array}$ \\
\cline { 2 - 7 } & \multicolumn{7}{|c|}{ Source Correlated Spectra } & \multicolumn{3}{c|}{ Total Spectra } \\
\hline \multicolumn{8}{|c|}{ Polyethylene Shielding DU } \\
\hline 3.93 & $2.4 \pm 0.8$ & 5.34 & 31.6 & $51.3 \pm 7.4$ & 1.30 & 6.6 \\
\hline 4.44 & $7.5 \pm 0.7$ & 12.63 & 2.1 & $158.3 \pm 6.6$ & 1.70 & 0.5 \\
\hline \multicolumn{8}{|c|}{ Aluminum Shielding DU } \\
\hline 1.81 & $6.9 \pm 0.8$ & 4.38 & 4.0 & $198.7 \pm 7.6$ & 2.27 & 0.5 \\
\hline 2.21 & $7.4 \pm 0.8$ & 4.30 & 3.2 & NA - Hydrogen interference \\
\hline 3.00 & $4.1 \pm 0.7$ & 4.27 & 7.4 & $185.2 \pm 5.7$ & 2.06 & 0.3 \\
\hline
\end{tabular}




\subsection{Investigation of Signature Induced from Fission}

It has been established that this technique can be used to specify a target and that polyethylene shielding DU can be identified by the carbon peak. Now the effects of enrichment and fission neutrons on this signature will be investigated.

\subsubsection{Simulations of Varying Enrichment}

Some simulations were done in MCNP PoliMi as an exploration of how uranium signatures vary with enrichment. This is an effect that we cannot look at experimentally. Experimentally, the spectrum associated with interrogating DU can be seen, but enriched uranium samples were not available to be measured. Figure 4.9 shows the results of $18 \mathrm{~kg}$ annular casting of uranium of different enrichments. The main difference between these two plots is the abundance of low energy photons, which makes sense due to the increased fission activity in the HEU object. Given the low efficiency of the HPGe detector, this increased number of photons is unlikely to overwhelm the detector experimentally. However, it is important to consider that gamma-ray peaks will likely have a reduced signal to background ratio, especially those below 2 or $3 \mathrm{MeV}$.

\subsubsection{Uranium vs Tungsten Signature Investigation}

The previous results show that certain shielding materials could be identified, which was a positive result. Given that this source correlated spectrometry technique has rarely been used for uranium characterization, a study was done of the signatures in the spectra from uranium. Three measurements were done for 20 minutes each: the DU casting without shielding, the DU casting with polyethylene 


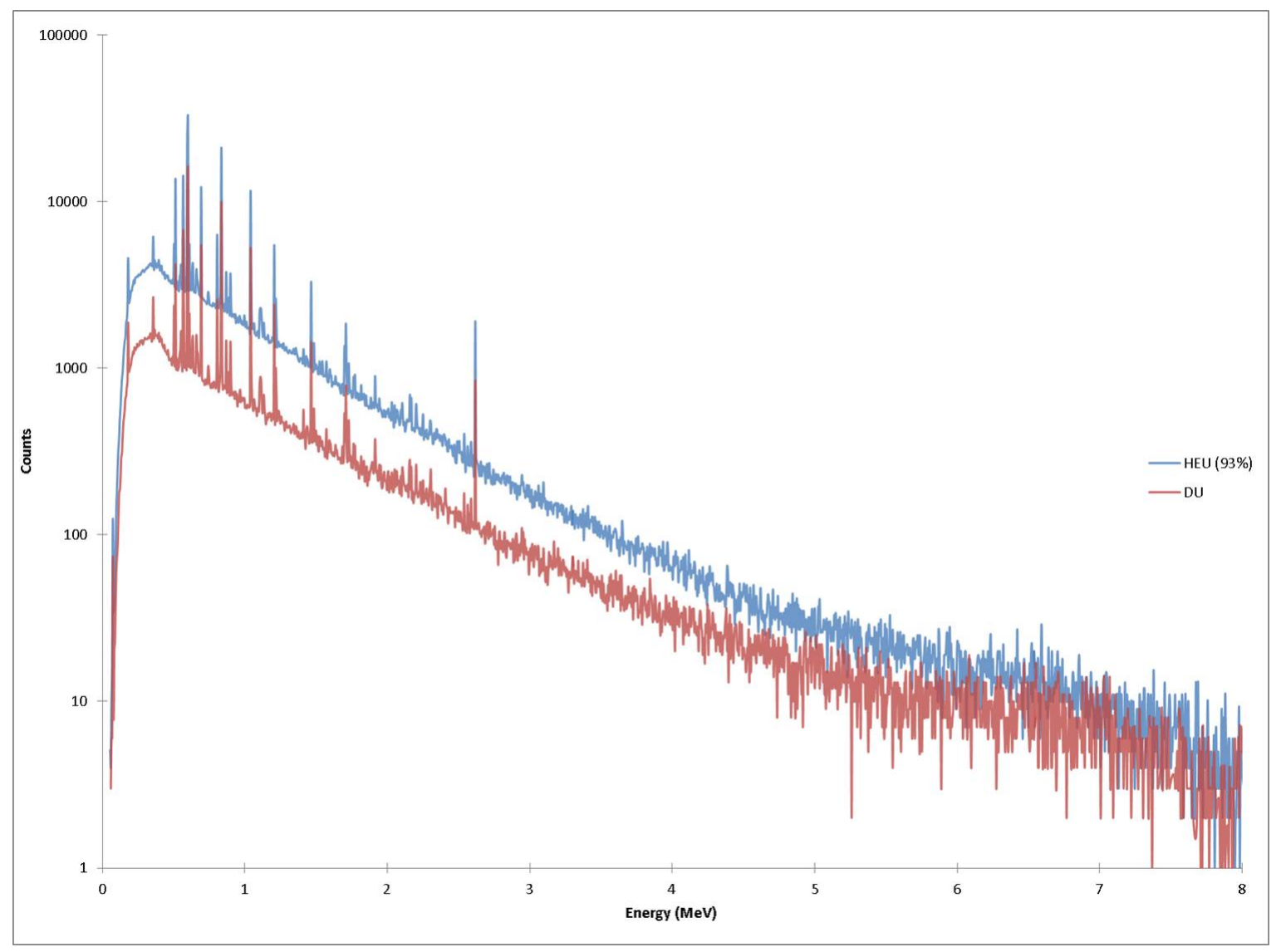

Figure 4.9: Simulations $14 \mathrm{MeV}$ prompt activated spectra of both DU and HEU.

shielding, and the tungsten casting with polyethylene shielding. This was done to see if any signatures can be seen from uranium itself, and to see if the carbon peak was different between DU and tungsten shielded by polyethylene. If the carbon peak were larger on the shielded DU spectrum as compared to shielded tungsten, it could signify fission neutrons activating the shielding material in a detectable way.

The results are shown in Figure 4.10. Any useful signatures from uranium itself would show up in both the blue and the green lines, but not the red line (which has tungsten instead of DU). There are a few peaks that are shared by all three measurements, meaning that they are likely from the germanium activation itself or other not useful signatures such as the $511 \mathrm{keV}$ peak. However, there do not 
appear to be any signatures above background that are unique to both the blue and green spectra. This suggests that this method is not useful for the identification of uranium material directly.

Comparing the carbon peaks of the shielded uranium and tungsten in Figure 4.10, it is not possible to visually determine a difference in the number of counts; the 4.44 MeV peak and the $3.93 \mathrm{MeV}$ peak both appear to be comparable.

Integrating both peaks for both inspection objects, the $4.44 \mathrm{MeV}$ peak builds at a rate of $7.5 \pm 0.7 \mathrm{cpm}$ for uranium and $5.3 \pm 0.7 \mathrm{cpm}$ for tungsten. The first escape peak builds at a $2.4 \pm 0.8 \mathrm{cpm}$ for uranium and $3.1 \pm 0.8 \mathrm{cpm}$ for tungsten. The 4.4 peak is higher for uranium than tungsten, though by a small margin. The escape peaks are comparable. The difference between the uranium and tungsten $4.4 \mathrm{MeV}$ peak is interesting, but not very convincing. Since those rates are low and the carbon peak already suffers from Doppler broadening, the next section will explore using this technique with a high efficiency NaI detector. 


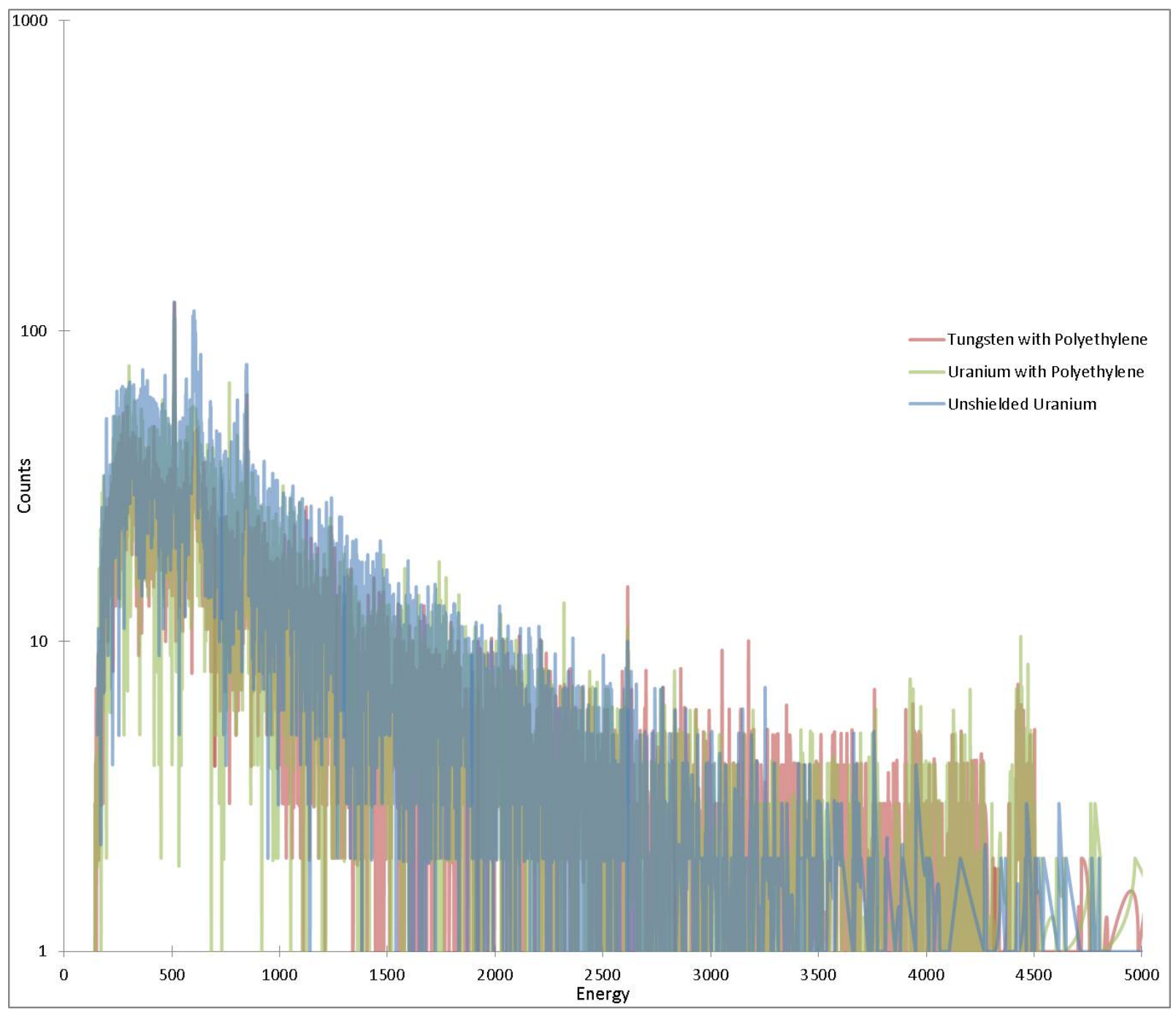

Figure 4.10: Exploration of uranium signatures in the spectra.

\subsection{Sodium Iodide Detector Measurements}

The next part of this work explored the possibility of performing a similar study with a NaI detector instead of an HPGe detector. The motivation for this was to see if the higher efficiency of the NaI detector would help the effort to identify the weak signature of fission neutrons activating carbon in polyethylene. Since the carbon peak is naturally broadened, the lower resolution of the NaI detector should 


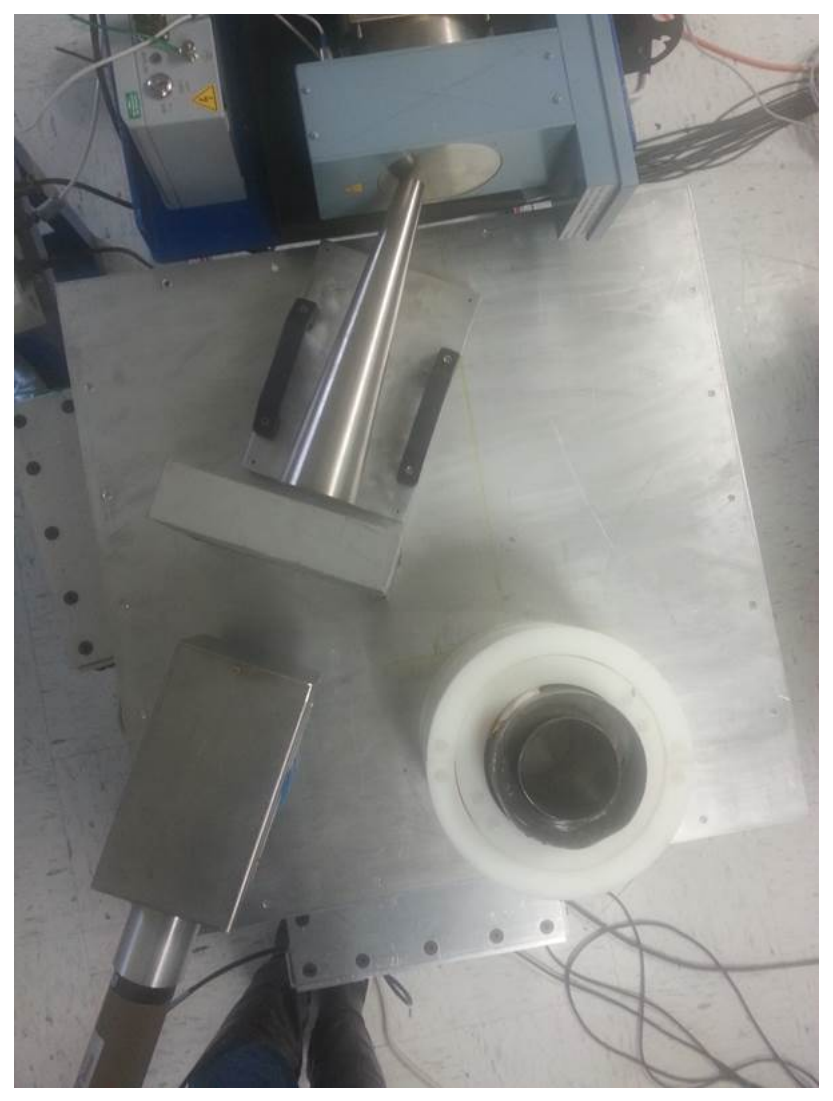

Figure 4.11: Experimental setup of NaI detector measuring spectra from polyethylene shielded DU

not be a concern.

\subsubsection{Experimental Setup}

The experimental setup is the same as described previously, but with the HPGe detector replaced by a $4 " \times 4 " \mathrm{NaI}$ detector with an attached photomultiplier tube (PMT). Figure 4.11 shows the setup with the NaI detector present. The detector is at an angle in order to be in the shielding cone of the tungsten shielding. Although damage to the NaI by source neutrons is not a concern for these measurements, the detector has much higher source neutron efficiency, which leads to the detector being overwhelmed by dead time from source neutrons if the source neutron rate 


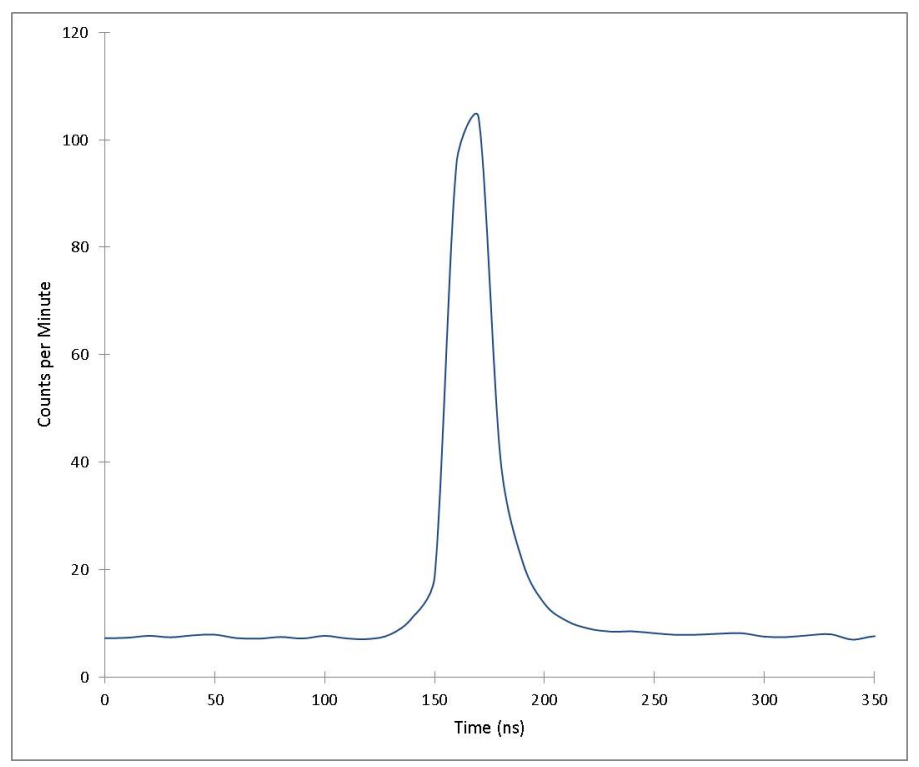

Figure 4.12: Correlation of the induced radiation detected in the HPGe detector following an detection in the alpha pixels (pixel 7 and 8 data summed).

in the detector is too high.

Due to the short decay time of the NaI, the correlation window is quite small. Figure 4.12 shows the correlation peak from the prompt radiation that is used for the correlation spectrum. Again, it should be noted that there are time differences due to cable length and pulse processing times that are not accounted for in the correlation plot, meaning that the location of the peak does not give time-of-flight information. From this plot, the correlation window used for the source correlated spectrum was $70 \mathrm{~ns}$ wide, from 130 to $200 \mathrm{~ns}$. A $70 \mathrm{~ns}$ window starting at 1 microsecond was taken to be the correlated background and was subtracted from the source correlated spectrum, just as was done with the HPGe spectra.

Figure 4.13 shows the pulse height spectrum of one of the source alpha pixels plotted on a log scale. Just as in Figure 4.4, the blue line is the total spectrum, not correlated to the NaI detector; the red line is all the pulse heights of the alpha pulses that fell into the time window shown in Figure 4.12; and the green line 


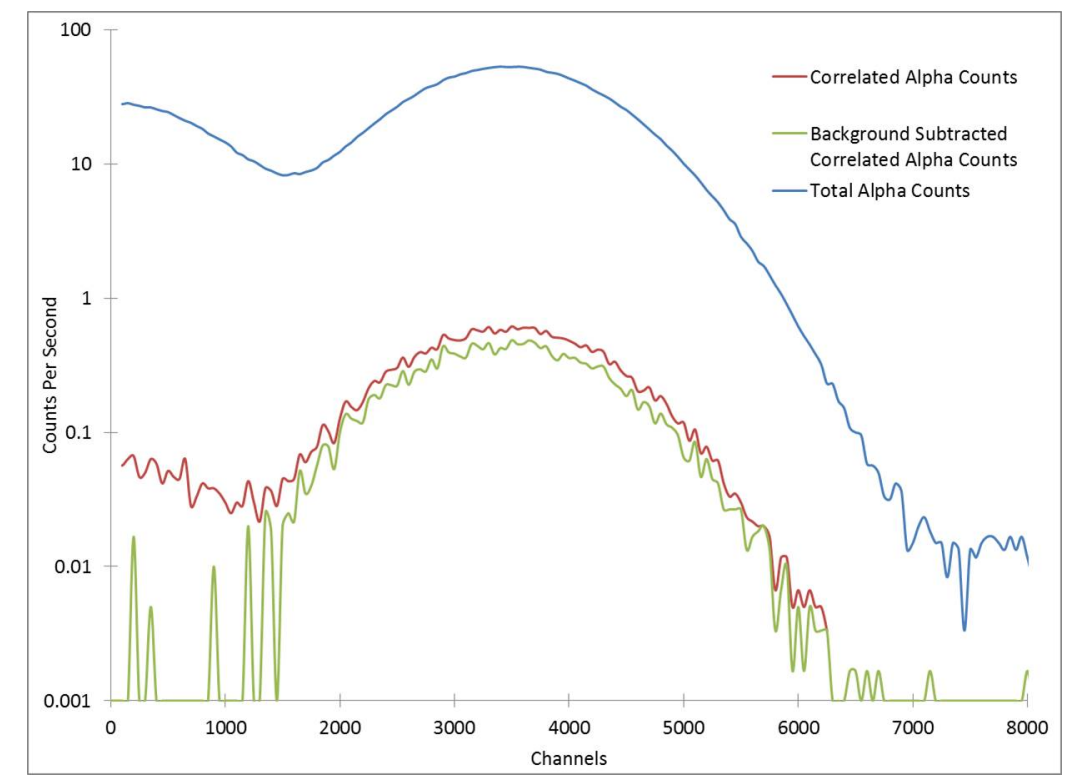

Figure 4.13: Pulse height spectra of the total alpha counts, alpha counts correlated to the NaI detector, and correlated alpha counts with background subtracted.

consists of data from the red line with the pulses in the background window subtracted out. In the background subtracted correlated spectrum, the lower end of the spectrum is almost completely gone, leaving only real alpha pulses remaining. This demonstrates the extremely low accidental correlation rate present in the source correlated spectrum, just as with the HPGe detector results.

\subsubsection{Identification and Localization of Material}

Just as with the HPGe detector, a measurement was done with the same three materials correlated to separate pixels. Again, the goal was to prove the ability to isolate materials in the field of view, and prove that no significant background peaks from surrounding materials contribute to the spectrum. The setup is the same as before, but with the NaI detector replacing the HPGe detector, as shown in Figure 4.14. The measurement was again taken for an hour.

The measurement results are shown in Figure 4.15. In the total NaI spectrum, 


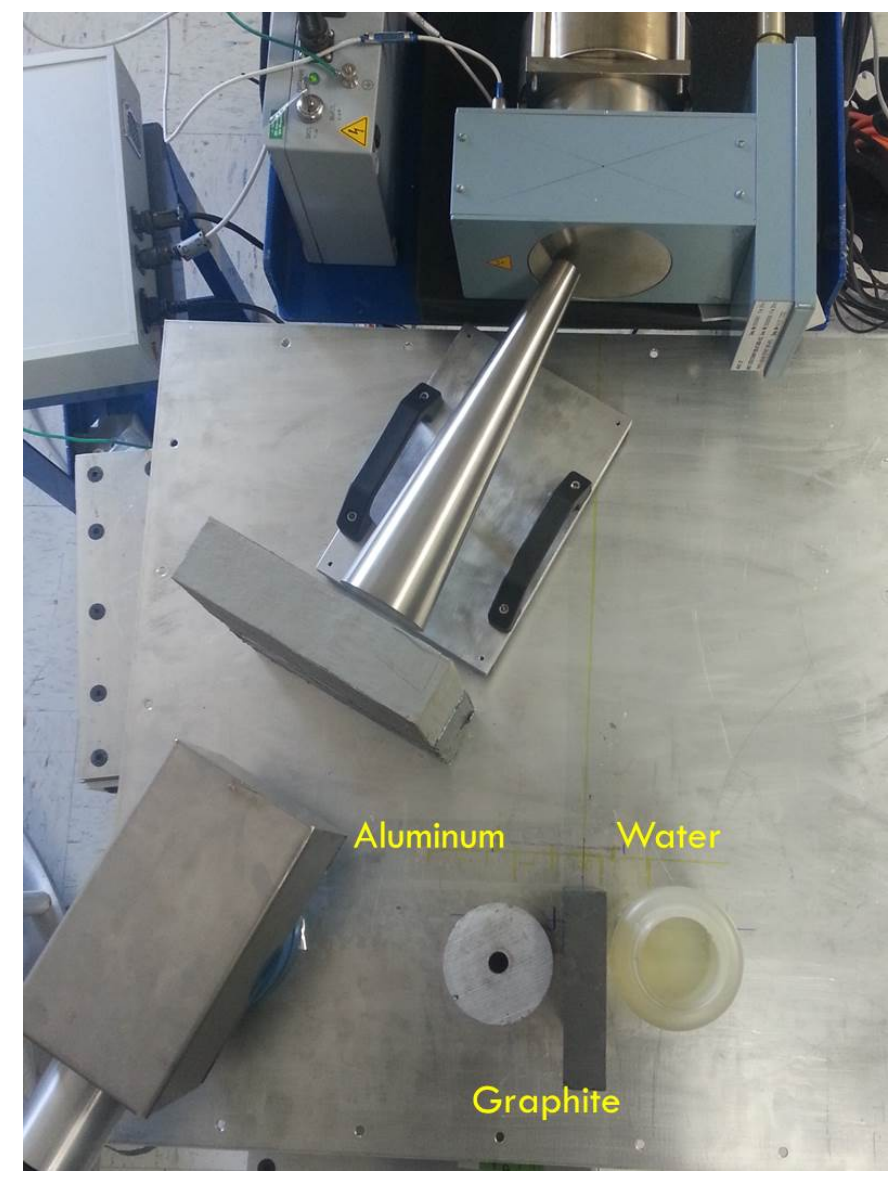

Figure 4.14: Experimental setup of measurement done to determine the ability of this technique to differentiate nearby materials using a $\mathrm{NaI}$ detector.

the lower energy peaks are a mixture of gamma rays from activation of surrounding materials and activation of the $\mathrm{NaI}$ itself. In the $1-3 \mathrm{MeV}$ energy range, a few aluminum peaks are slightly visible (1.8 MeV, 2.2 MeV, and 3.0 MeV) from the aluminum surface in front of the generator, along with the hydrogen capture peak mostly contributing to the $2.2 \mathrm{MeV}$ peak from the polyethylene, $2.6 \mathrm{MeV}$ gammarays from the lead shielding present, and finally carbon along with its first escape peak at $4.4 \mathrm{MeV}$ and $3.9 \mathrm{MeV}$ respectively.

In the correlated spectra, the self attenuation and lowered efficiency and resolution for higher energy photons are again seen in these three spectra. The aluminum 
peaks seen by pixel 6 are again very evident. Given the already poor resolution of $\mathrm{NaI}$, the physical effects that widen the carbon peaks are no longer evident, so the carbon peak and escape peak are very evident. The oxygen peak is not possible to resolve, though it is clear that the counts in the 5-6 MeV range are elevated, this would not be enough to identify the presence of a target containing oxygen. 


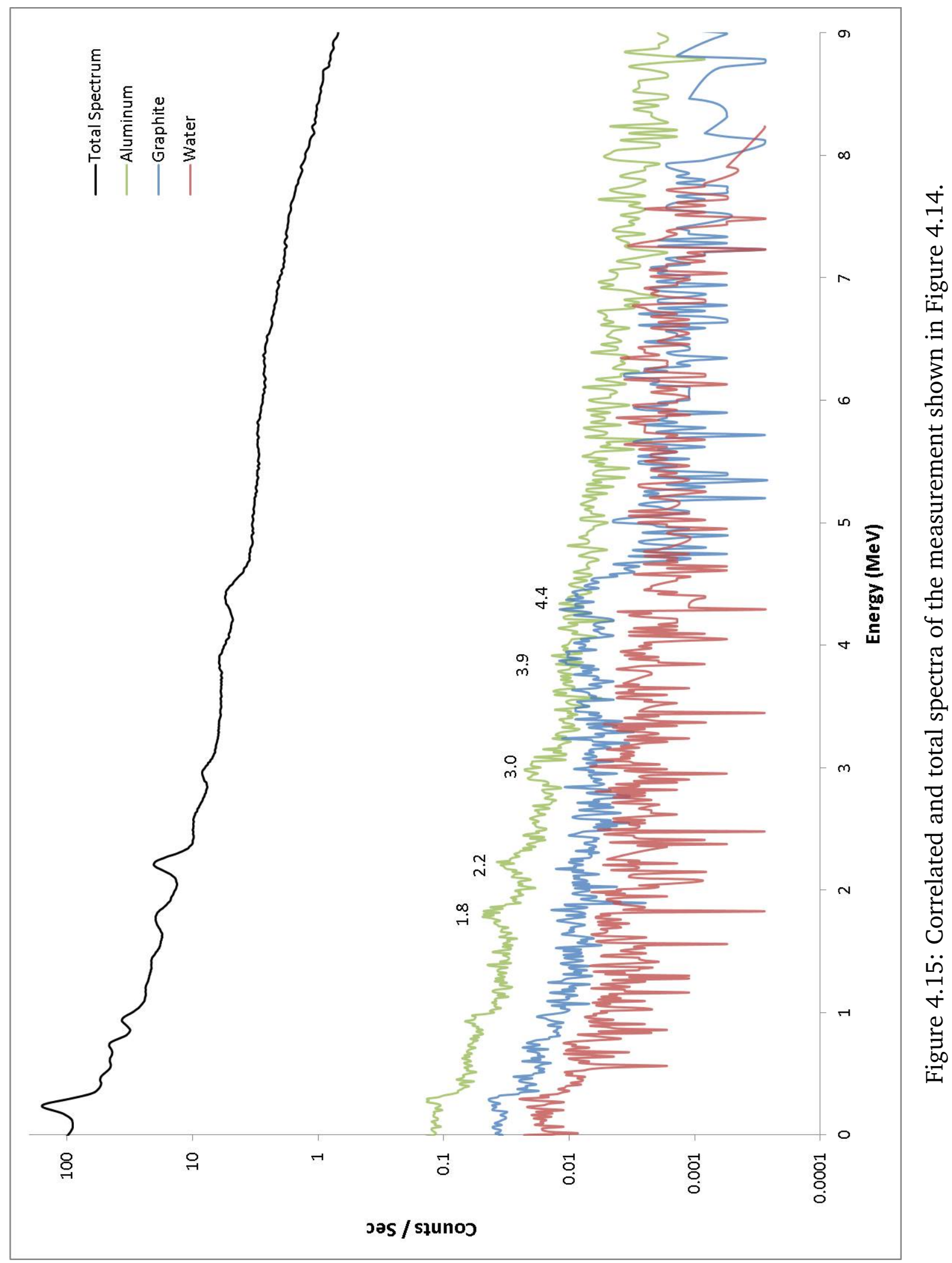


Table 4.4 shows a summary of the results, using the NaI detector. As seen in Table 4.4, the total spectra consistently have a shorter time until detection due to the vastly superior statistics. However, the peak to background (BG) was consistently higher for the correlated spectra, except for the oxygen peak that could not be seen. It is also very interesting that both carbon and aluminum could be detectable with a single pixel in less than 10 minutes. Utilizing three pixels, the first escape peak is even identifiable in under 10 minutes.

Given the drastic reduction in precision, it is interesting that the NaI still gave quite good results. This results show that materials can still be isolated very effectively within the pixel view. These results also do show that the the carbon peaks are around $2 \times$ as strong with the higher efficiency of the NaI detector. Therefore all results were positive.

Table 4.4: Integrals of the peaks of interest in counts per minute (CPM) from spectra shown in Figure 4.15

\begin{tabular}{|c|c|c|c||c|c|c|}
\hline \multirow{2}{*}{$\begin{array}{c}\text { Energy } \\
\text { (MeV) }\end{array}$} & CPM & $\begin{array}{c}\text { Peak to } \\
\text { BG Ratio }\end{array}$ & $\begin{array}{c}\text { Time Until } \\
\text { Detectable } \\
\text { (min) }\end{array}$ & CPM & $\begin{array}{c}\text { Peak to } \\
\text { BG Ratio }\end{array}$ & $\begin{array}{c}\text { Time Until } \\
\text { Detectable } \\
\text { (min) }\end{array}$ \\
\cline { 2 - 7 } & \multicolumn{7}{|c||}{ Source Correlated Spectra } & \multicolumn{3}{c|}{ Total Spectra } \\
\hline \multicolumn{7}{|c|}{ Pixel 8 - Aluminum } \\
\hline 1.81 & $12.1 \pm 1.5$ & 1.76 & 9.9 & $2704.3 \pm 35.5$ & 1.24 & 0.1 \\
\hline 2.21 & $6.7 \pm 1.4$ & 1.70 & 26.8 & NA - Hydrogen interference \\
\hline 3.00 & $5.6 \pm 0.9$ & 1.71 & 17.6 & $548.7 \pm 12.0$ & 1.17 & 0.9 \\
\hline \multicolumn{7}{|c|}{ Pixel 7 - Graphite } \\
\hline 3.93 & $2.9 \pm 0.6$ & 1.95 & 25.3 & $541.4 \pm 16.8$ & 1.13 & 0.6 \\
\hline 4.44 & $5.3 \pm 0.6$ & 3.19 & 6.3 & $961.4 \pm 16.5$ & 1.30 & 0.2 \\
\hline \multicolumn{7}{|c||}{ Pixel 6 - Water } \\
\hline 5.61 & \multicolumn{7}{|c||}{$106.8 \pm 12.5$} & 1.09 & 8.8 \\
\hline 6.13 & Not Detectable & $144.7 \pm 10.2$ & 2.36 & 3.2 \\
\hline
\end{tabular}




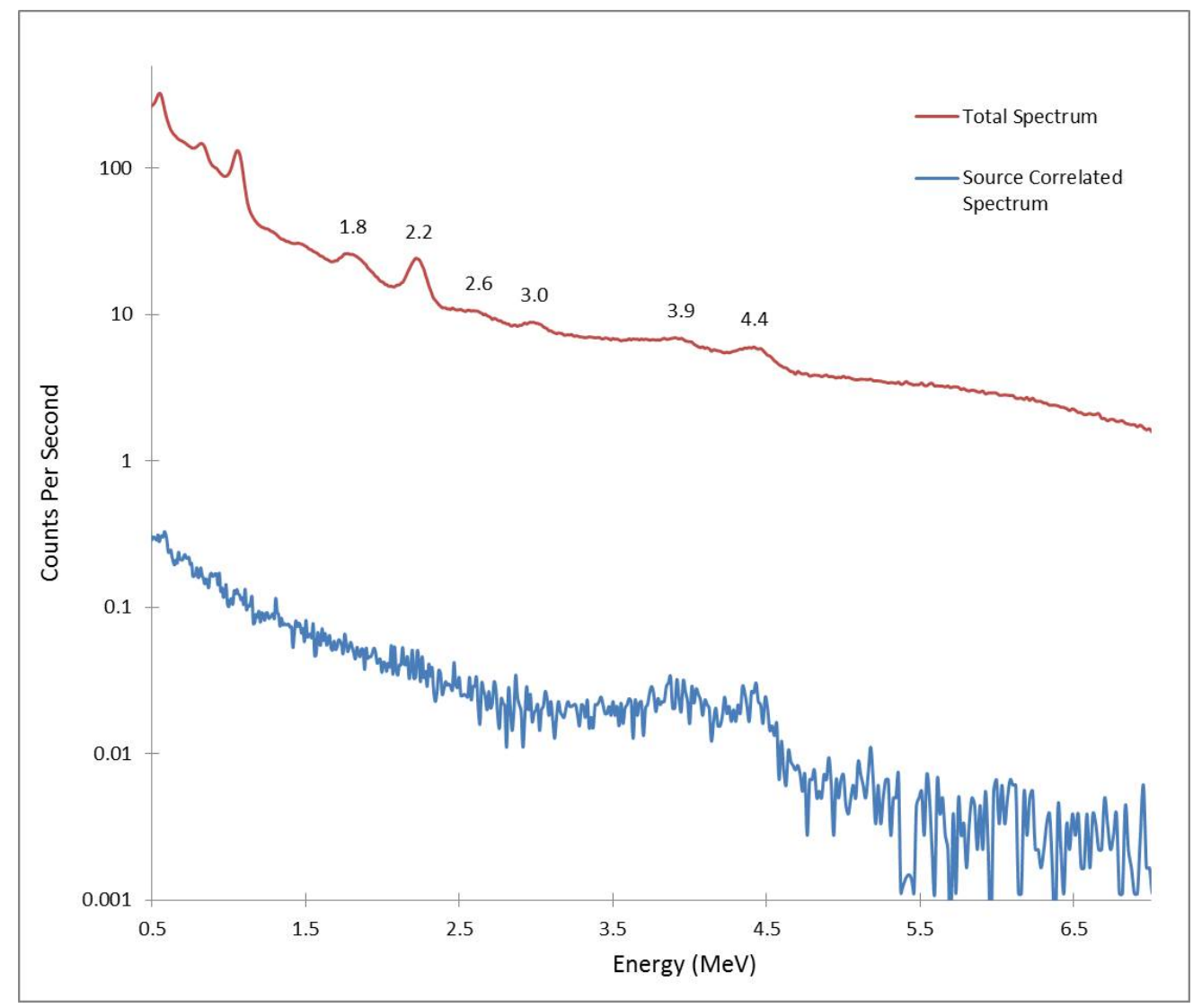

Figure 4.16: Correlated and total spectra of polyethylene shielded DU.

\subsubsection{Investigation of Signatures from Shielded Uranium}

Again, the signatures from polyethylene shielded uranium are investigated, but now with the NaI detector. The target, shown in Figure 4.11, consists of an $18 \mathrm{~kg}$ DU casting and $5 \mathrm{~cm}$ of shielding material surrounding it. The measurement was taken for 30 minutes, with the DT neutron generator outputting between $9 \times 10^{6}$ and $1 \times 10^{7}$ neutrons per second.

Figure 4.16 shows the total NaI spectrum and the source correlated spectrum for the measurement plotted on a log scale. Again, the total spectrum has various characteristic gamma-ray peaks, but since the field of view of the three alpha pixels that are correlated to the NaI detector only includes the target object, all the peaks 
go away except for the carbon peaks. The carbon peaks also become easier to see due to the severely reduced background. The integrated peak results can be seen in Table 4.5.

Table 4.5: Integrals of the peaks of interest in counts per minute (CPM) from spectra shown in Figure 4.16

\begin{tabular}{|l|c|c|c||c|c|c|}
\hline \multirow{2}{*}{$\begin{array}{c}\text { Energy } \\
\text { (MeV) }\end{array}$} & CPM & $\begin{array}{c}\text { Peak to } \\
\text { BG Ratio }\end{array}$ & $\begin{array}{c}\text { Time Until } \\
\text { Detectable } \\
\text { (min) }\end{array}$ & CPM & $\begin{array}{c}\text { Peak to } \\
\text { BG Ratio }\end{array}$ & $\begin{array}{c}\text { Time Until } \\
\text { Detectable } \\
\text { (min) }\end{array}$ \\
\cline { 2 - 6 } & \multicolumn{7}{|c|}{ Source Correlated Spectra } & \multicolumn{3}{|c|}{ Total Spectra } \\
\hline \multicolumn{7}{|c|}{ Polyethylene Shielding DU * } \\
\hline 3.93 & $10.3 \pm 1.6$ & 1.82 & 7.0 & $566.5 \pm 25.4$ & 1.11 & 0.6 \\
\hline 4.44 & $10.9 \pm 1.4$ & 2.47 & 4.8 & $899.7 \pm 22.9$ & 1.21 & 0.2 \\
\hline
\end{tabular}

\subsubsection{Activation of Polyethylene from Fission Neutrons}

Figure 4.17 shows a measurement that was made to explore the amount of signal that is induced from the source neutrons as compared to the amount of signal induced from the fission and scattered neutrons. A polyethylene block was placed outside of the field of view of the pixels, but between the NaI detector and the uranium casting, so that there is a flux of fission neutrons into the polyethylene that is correlated to the source. One measurement was taken without the block present, and the next was taken with the block present.

Previous measurements have proven that the signatures in the field of view have very little contribution from surrounding material. Therefore, any carbon peaks that are seen with the polyethylene block present must be from the fission neutrons. Based on measurements of the polyethylene shielded uranium, it is clear that the carbon peak is detectable, but not clear if any of this peak originates from fission neutrons activating the carbon. This experiment will provide clarity. 


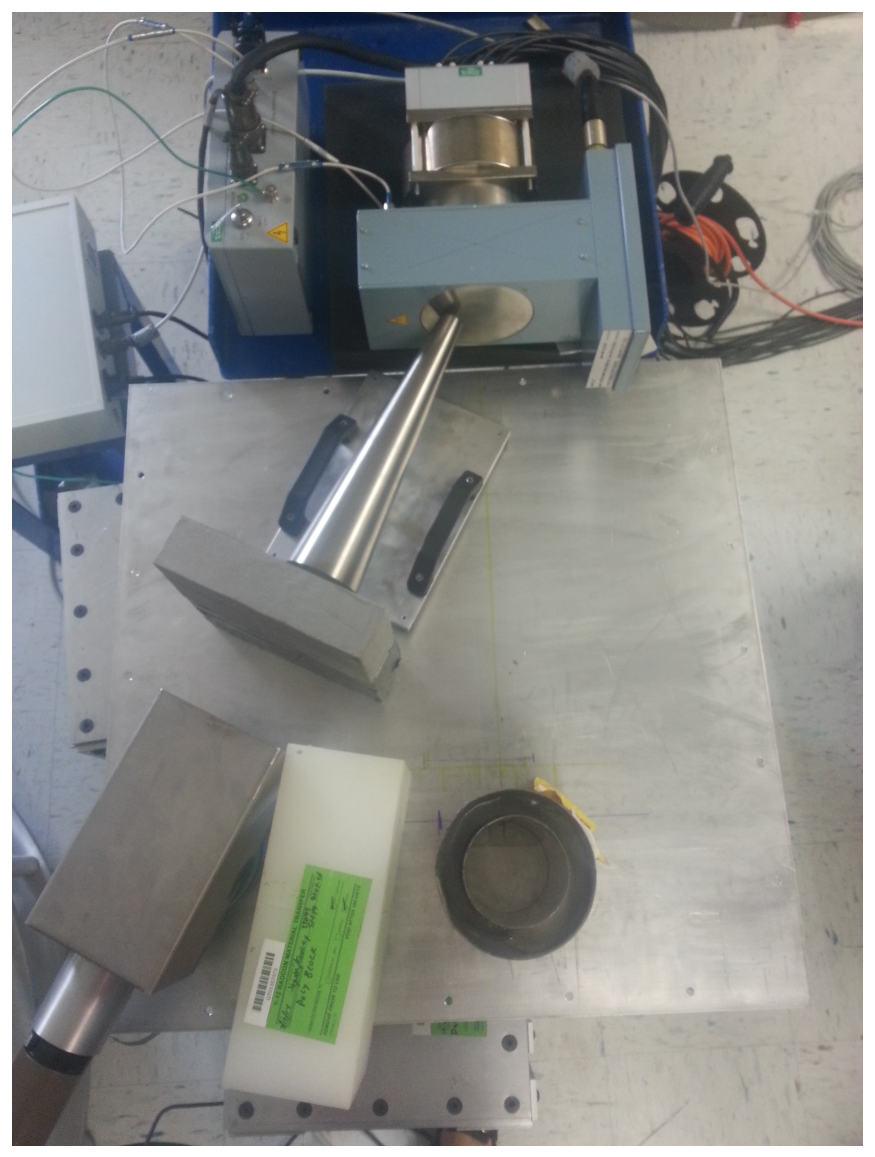

Figure 4.17: Experimental setup to investigate the contribution from fission neutrons inducing gamma-rays.

The results are shown in Figure 4.18. The spectra show the same shape, with some attenuation from the polyethylene block, especially at lower energies. This suggests that very little of the spectra occurs from fission neutrons inducing gammarays in the material. Also, the carbon signatures from the block cannot be seen in the correlated spectrum. This means that the fission neutrons are not producing a detectable amount of activation within the polyethylene. 


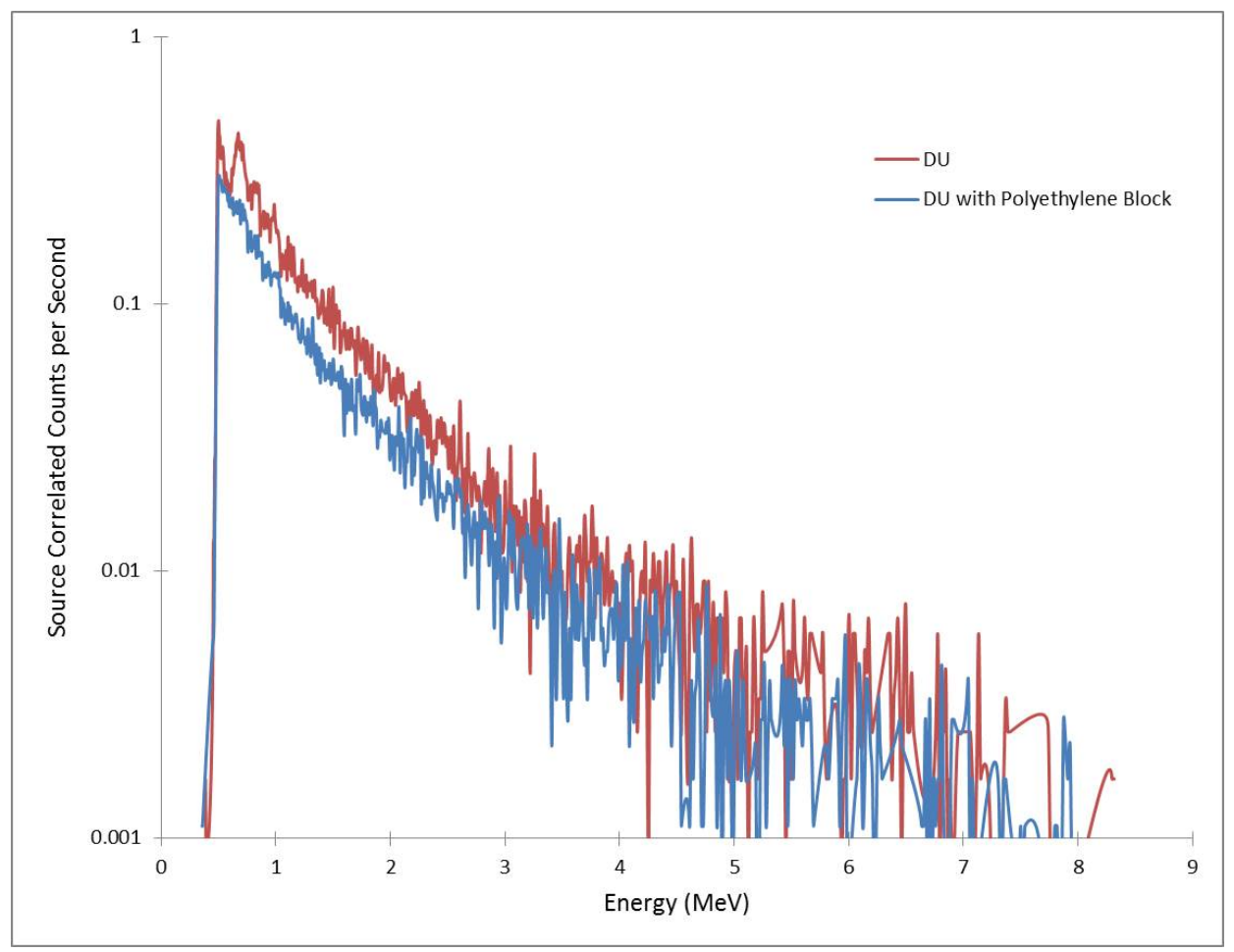

Figure 4.18: The resulting spectra from the measurement shown in Figure 4.17, with and without the polyethylene block present.

\subsection{Discussion}

The results from this series of measurements show that it is unlikely that the activated carbon peak could be related to the level of enrichment. No measurements were able to prove convincingly that activated carbon by fission neutrons is detectable. Much of the fission neutron energy spectrum is not high enough to activate the $4.44 \mathrm{MeV}$ carbon peak, so it is not surprising that this phenomenon would be too weak to detect. If this signature had been detectable, it could have potentially be used to determine enrichment in polyethylene shielded uranium, which is a difficult problem.

This explorations leading up to looking for this fission signature gave positive results, showing the usefulness of source correlated prompt gamma-ray spectrom- 
etry towards the goal of identifying and localizing material. The positive results are not surprising, given the related research has been done by other groups, as discussed previously. However, many of the previous studies focused on bulk items, correlating larger pixel segments to much larger target objects such as shipping containers.

The ability of this technique to identify isotopes and filter out background, even differentiating between nearby materials, was very good. Both detector types proved the ability to identify certain materials with a relatively short measurement time, which could be easily shortened further by adding detector efficiency. The HPGe detector offers more options for isotope identification, due to its vastly superior resolution. However, it was interesting that the cheaper, more rugged NaI detector was able to pick the small amounts of gamma-ray signatures from the target items out of vast amount of background.

There are additional ideas that could be explored to expand on this study. For example, this work could be extended by using a digitizer with higher timing resolution to explore the ability to differentiate materials at different depths within a material by relating the time-of-flight of the signature to the penetration of neutrons in the object. 


\section{5 | Conclusion}

The goal of this series of studies was to investigate several signatures and analysis techniques for characterizing the enrichment and multiplicative properties of shielded uranium, the main commonality between all studies being the use of an API-DT neutron generator. The main question being asked by this work was: are photon signatures from an API-DT generator useful for determining enrichment of a shielded uranium object? The study included photon-only signatures and photon signatures in combination with neutron signatures. While this was not an exhaustive study of all possible photon signatures from an API-DT neutron generator interrogating shielded uranium, some conclusions can be drawn about the photon signature strengths and weaknesses.

Some of the main conclusions from the specific studies were as follows:

- The time-of-flight sorted signatures investigated in Chapter 2 were all sensitive to enrichment. However, the source-photon signature could not differentiate DU from the benign tungsten, unless in combination with a higher order photon correlation signature (e.g. source-photon-photon).

- Also in Chapter 2, the source-photon-neutron signature was especially interesting because of its ability to be closely modeled by the MCNP simulation tool, and its sensitivity to enrichment across different types of shielding materials. For enrichment determination in shielded uranium via comparison to MCNP-PoliMi simulations, this work found that the source-photon-neutron 
signature is the most reliable.

- Improvements in the methods for measuring the API-DT detection system parameters and modeling those parameters in MCNP simulations is needed. A rigorous uncertainty investigation is needed to better understand the sources of uncertainty contributing to differences between the simulation and data.

- In Chapter 3, the point kinetics equations did an impressive job of modeling the signatures escaping uranium spheres. The photon signatures proved to be impressively closely matched between point kinetics and MNCP simulations in the presence of large amounts of polyethylene shielding.

- Also in Chapter 3, the signatures were still useful in differentiating between DU and HEU when some reasonable uncertainty values were propagated through the equations. For the photon signatures, the geometric uncertainties have a large effect on the ability of the signature to predict enrichment because of the large amount of self-attenuation.

- In Chapter 4, the photon signatures were excellent at identifying and localizing certain types of materials. However, using this signature to inform about enrichment in polyethylene shielded uranium was not possible using the APIDT generator with one spectrometry detector.

By the end of this dissertation, the energy, timing, and number distribution of photon signatures have all be explored. Based on this information, some generalized conclusions can be drawn about the $14 \mathrm{MeV}$ induced prompt photon signatures deriving from shielded uranium.

- Most of the photons deriving from uranium, both from fission and non-fission interactions, are low energy gamma-rays (generally less than 1-2 MeV), which 
allow for high energy gamma-rays signatures from non-uranium materials to be distinguished via spectrometry.

- The signature is complex, since it includes both fission gamma-rays as well as gamma-rays from other non-elastic neutron interactions and photon interactions. While neutrons are rarely produced in groups of more than two except in fission interactions, photons are often produced in cascades, making the fission interactions more difficult to identify based on coincidences.

- Self attenuation in uranium is a very significant factor in the rate of photons escaping a uranium configuration, and adds to the complexity of the photon signature.

- Given the high self attenuation rates, the accuracy and precision of the geometry information are very important inputs into any model of dense material that is predicting photon signatures. High levels of uncertainty in geometry information will likely lead to these models giving poor results.

- Despite the complexity of the photon signatures, they still may be useful for uranium shielded by low-Z materials that are effective neutron shields. Unfortunately, low-Z material also degrades neutron radiography, which the models of the photon signature are heavily reliant on.

While this series of studies has provided some information about the photon signatures from shielded uranium detected by API-DT detection systems, there is still plenty to be explored on this topic. These signatures have not been researched extensively, leaving plenty of future work. The most positive results from this series of studies was the utility of the source-photon-neutron signature, and the ability of the simple point kinetics model to predict the photon moments of uranium shielded 
by large amounts of polyethylene. These positive results motivate future studies of these signatures. Some future studies might include:

- A thorough uncertainty analysis of a API-DT detection system, including imaging capabilities and SNM characterization capabilities.

- A non-empirical method for predicting photon absorption probabilities of photons in various geometries of uranium. The lack of a reliable method for determining this probability was a weakness of the analysis in Chapter 3.

- An investigation of using a DD neutron generator in place of an DT neutron generator for the analysis done in Chapter 3. This would reduce the number of inputs into the model.

- A comparison of the model in Chapter 3 with a detection system optimized to reduce dead time and cross talk. 


\section{A | Detector Position Sensitivity Char- acterization}

\section{A.1 Overview of Study}

The purpose of the study presented in this appendix was to explore the position sensitivity of the light response reaching the PMT in plastic detectors used throughout this dissertation. In the past, when comparing the data from NMIS to simulations done in PoliMi and parsed by the post processor written by B. Grogan (39) and J. Henkel, there have been some large differences. In general, it has been noted that small detectors are much better modeled by simulations than larger detectors (34). It has also been observed that thresholds may not be modeled properly.

While the light response of the detectors is modeled in the post processor used to parse the PoliMi output files, the scintillation light is not transported in the simulations. The post processor assigns a scintillation magnitude to each interaction that occurs in the detector based on the type of interaction and energy released, using light curves determined experimentally. Then scintillation magnitude is given some Gaussian uncertainty and the total light from all interactions in a short period of time is summed to represent a detector pulse. Lastly, the program checks that the total pulse magnitude is over the threshold. Experimentally, the amount of scintillation light that reaches the PMT will be a function of many factors, such as 
Table A-1: Definitions of all detectors used in this research

\begin{tabular}{|c|ccc|}
\hline Detector & Type & Size & HV \\
\hline Small & Scionix V25.4B102 & $1^{\prime \prime} \times 1 " \times 4 "$ & $-850 \mathrm{~V}$ \\
Medium & Bicron Mod:3.75x3.75 H4BC420/3L & $4^{\prime \prime} \times 4^{\prime \prime} \times 4.6^{\prime \prime}$ & $+1425 \mathrm{~V}$ \\
Large & Scionix Holland Serial SAI225 & $9.8^{\prime \prime} \times 9.8^{\prime \prime} \times 3.2^{\prime \prime}$ & $-1450 \mathrm{~V}$ \\
Very Large & Eljen M550-40x118x118 & $11.8^{\prime \prime} \times 11.8^{\prime \prime} \times 4.1^{\prime \prime}$ & $-1900 \mathrm{~V}$ \\
\hline
\end{tabular}

- energy deposited into the detector,

- the solid angle of the PMT with respect to the origin of the scintillation,

- the amount of reflection that contains the scintillation light in the detector volume, and

- the amount of attenuation of scintillation light within the detector.

To explore how the scintillation light varies, detectors were scanned by narrow cones of mono-energetic radiation to pin point the response from specific locations within the detector. Detectors of several different sizes were scanned, to investigate the effect of detector size on varying scintillation response. A correlated $14 \mathrm{MeV}$ neutron cone was used in the first study, and an intense ${ }^{137} \mathrm{Cs}$ source collimated by a block of tungsten with a pinhole was used in the second study. The detector sizes investigated are defined in Table A-1.

The light response of scintillation detectors has been researched by many different groups. A good summary of fast neutron scintillation detector topics is given by Klein and Brooks (67). The most similar study to the one presented here was done by Naeem, Clarke, and Pozzi (68), who used a collimated ${ }^{137} \mathrm{Cs}$ source and a collimated ${ }^{252} \mathrm{Cf}$ source to explore the spatial response of a liquid scintillation detector. Their results were similar in some ways to the results here, but they discussed the unique challenge of the expansion in volume of liquid scintillators, which is not 
applicable here. This work is unique for using a monoenergtic neutron source with associated particle capabilities, and exploring detectors of different sizes.

\section{A.2 $14 \mathrm{MeV}$ Detector Characterization}

\section{A.2.1 Experimental Setup}

An API 120 DT generator was mounted on the movable arm of NMIS with a PMT mounted to the alpha detector face plate. Between the PMT and the face plate was a black opaque piece of plastic with a $3 \mathrm{~mm}$ diameter circle in the center of it. This created a narrow correlated neutron cone that was used to probe small areas of the detector at a time. The alpha detector threshold was set, and the correlated neutron cone was characterized in the same method described in Chapter 4. It was determined that the correlated neutron cone was about $8^{\circ}$ and fairly circular, so that when the detectors were placed $11 \mathrm{~cm}$ away from the neutron source, the source spot had a diameter of about $1.5 \mathrm{~cm}$ (this cone included about $75 \%$ of correlated neutrons).

Two measurement series were conducted to investigate detector response. First the detector response was recorded with the NMIS analog system by scanning every inch of all detectors. Starting along one edge, the detector was placed on a table 11 $\mathrm{cm}$ from the DT generator, and the DT generator arm was moved to automatically take a series of short measurements from the bottom edge to the top edge, taking 1 inch steps. Then the detector was moved so that the generator was aligned with with the next horizontal inch of the detector and the vertical scan was repeated, until every square inch of the detector was scanned.

These results were compared to simulations of this process to see that the de- 
tector responses being modeled matched the simulated response. For the very large detector, the results were not used in analysis due to dead time issues. Throughout the measurements, the CFD threshold on all detectors were set low, equivalent to a neutron energy of about $0.75 \mathrm{keV}$. One measurement was done with the large detector with a high threshold, equivalent to CFD threshold of about 2.6 MeV neutrons.

The second study switched to a digital analysis of the detector responses. A few select locations in the detectors were measured with a CAEN V1730 digitizer. The V1730 is a 14 bit, 500 MS/s 16 channel digitizer running DPP-PSD firmware. Data was taken using a specialized version of the DPP-PSD software that was capable of correlation plots between channels, which was provided by Carlo Tintori, the CTO of CAEN. The anode signals of the detector and DT generator alpha detector were run through the digitizer to choose appropriate timing and pulse shape settings, and then data was taken in list mode and analyzed offline.

\section{A.2.2 Results}

The analog scans of the small, medium, and large detectors gave close match between the measured and simulated correlated count rates. Figure A-1 shows the results of the vertical scan of the middle of the large detector, both simulated and measured. The results are very close, meaning that the $14 \mathrm{MeV}$ neutron response is likely modeled well. However, the large detector was also scanned with the threshold raised from $40 \mathrm{mV}$ to $100 \mathrm{mV}$, which is equivalent to raising the threshold from a minimum neutron energy of $0.75 \mathrm{MeV}$ to $2.6 \mathrm{MeV}$. The results in Figure A-2 show that when the threshold is raised, the simulations no longer match the data as well. 


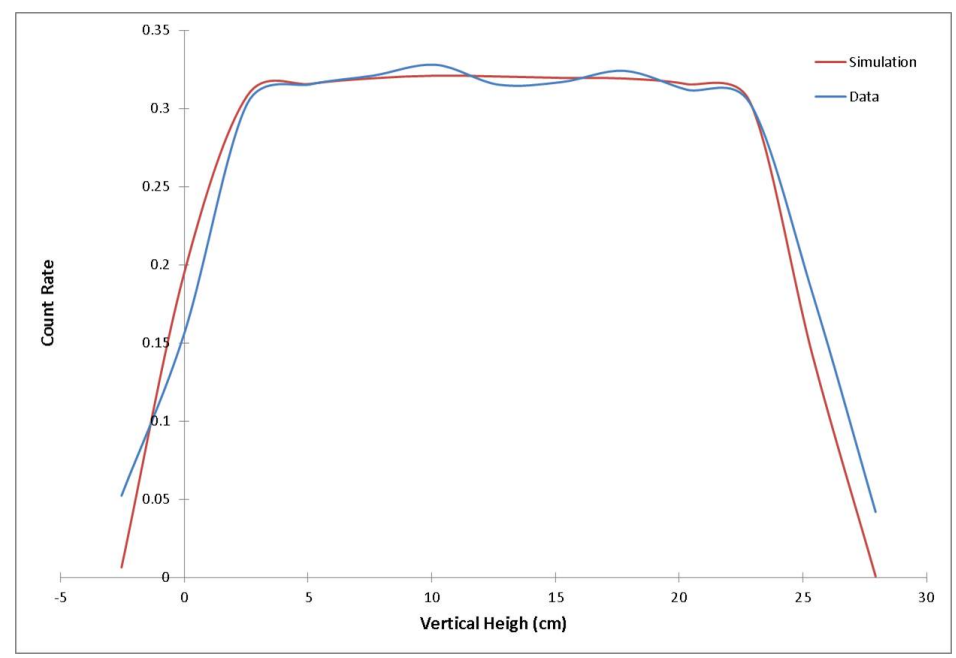

Figure A-1: Vertical scan of middle of large detector with low threshold, comparison of data and simulation.

When looking at the detector response across the surface of the simulated and measured detectors, all detectors are fairly uniform across the detector face, and the simulations and experimental detector response match well. For the scan done for the raised threshold of the large detector, some position specific non-uniformities are heightened. Figure A-3 shows a color map of the detector response of the interaction location for the high threshold large detector, comparing data to simulations. The simulated detector face is uniform throughout the middle of the detector, and drops off along the edges due to part of the neutron beam not hitting the detector face. The experimental detector face shows certain areas of the detector which respond more sensitively than others. However, even in this high threshold version, the neutron energies hitting the detector are well above the threshold, and the variation of response across the detector face is very slight.

The results from the digitizer analysis give further insights into variation of detector responses. Figure A-4 shows the results of the digitizer response for a few locations within the detectors. In each figure, the box containing the spectra is sup- 


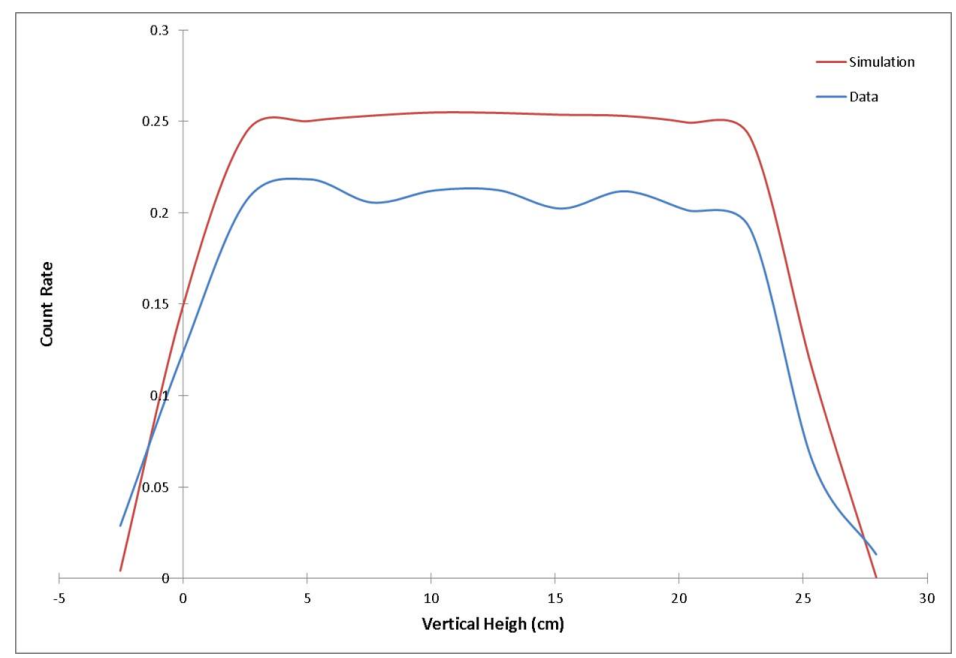

Figure A-2: Vertical scan of middle of large detector with high threshold, comparison of data and simulation.

posed to represent the face of the detector that was scanned, and the location that each spectrum is placed within the detector face is supposed to give an idea of the location that the spectrum was taken. The location of the PMT and the tabletop are also marked in each figure, for further reference of the location of the spectrum with respect to detector orientation.

The detectors used are all different models and manufacturers, so direct comparison between detectors is not informative. The goal of this investigation is to look at the variation of the responses within each detector, as opposed to comparing responses between detectors. It was sufficient to use channel number for this reason.

The results are interesting because it does appear that the location of the interaction within the detector varies quite a bit in the large detector, where as the small detector is fairly uniform. The medium detector has some variation, having a higher, less defined Compton edge near the PMT. The large detector's variation was similar, but with a higher Compton edge in front of the PMT and low Compton 

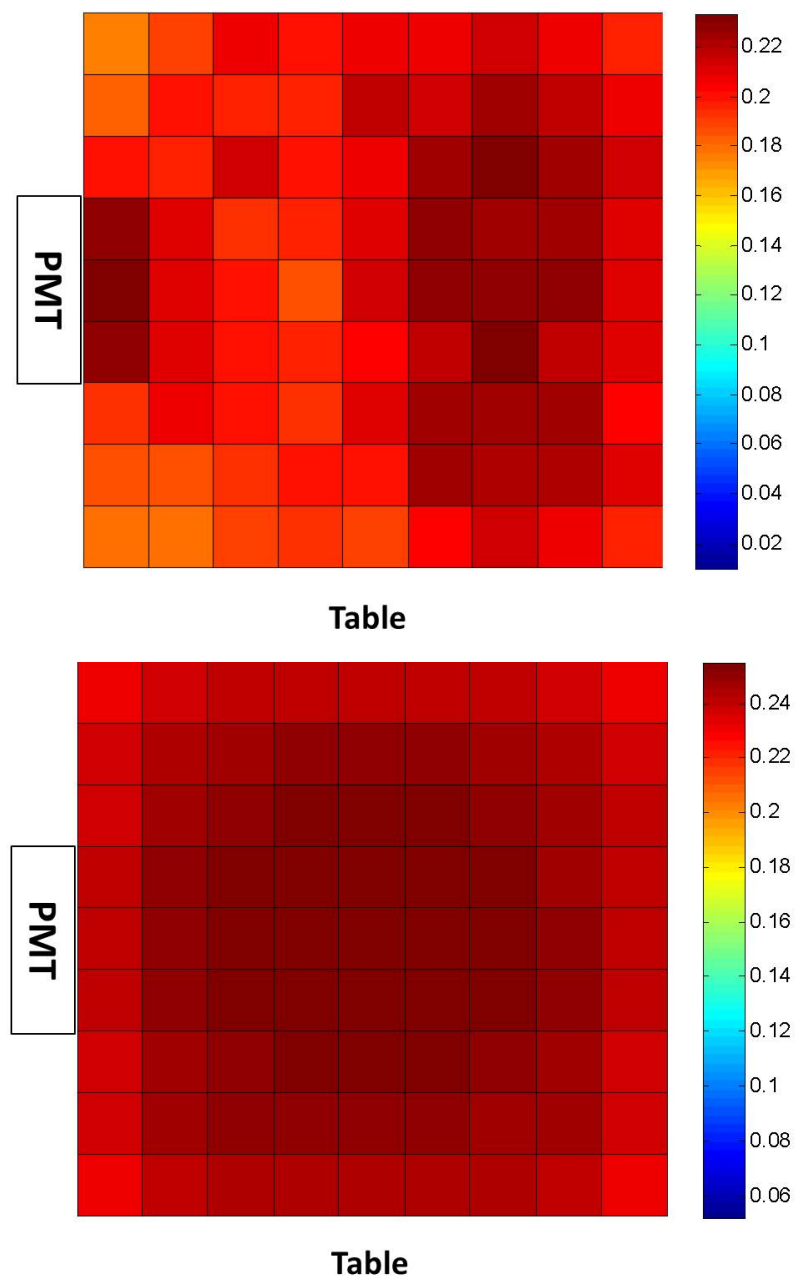

Figure A-3: Color mapped response of detector face with high threshold, comparison of simulation (right) and data (left). Units are in counts per source detection.

edges in the corners nearest the PMT. This makes sense, because the PMT would be able to "see" the interactions in front of it the best, and where as most of the light from interactions in the corners nearest the PMT would only reach the PMT by reflecting inside the detector. The manner in which the light varies across locations was studied in more detail with the ${ }^{137} \mathrm{Cs}$ source in the next section.

Comparing these results to the simulations, seen in Figure A-5, the spectra are predictably different. Since the scintillation light is not being transported through the material, the responses look uniform throughout the detector face. 
Again, these detectors are from different manufacturers and cannot be directly compared. However, it is still interesting to get a sense for the amount of variation of pulse height distribution with interaction location within the detector. Figure A- 6 gives a sense of the amount of variation, comparing the distribution of counts for each spectrum at each location across the channel number. The amount of spread between lines is shaded in blue to draw attention to the variation between lines instead of the individual lines themselves. The amount of spread also informs about the CFD thresholds position sensitivity for different detectors. Based on this series of plots, the variation of pulse heights does appear to increase with detector size. 

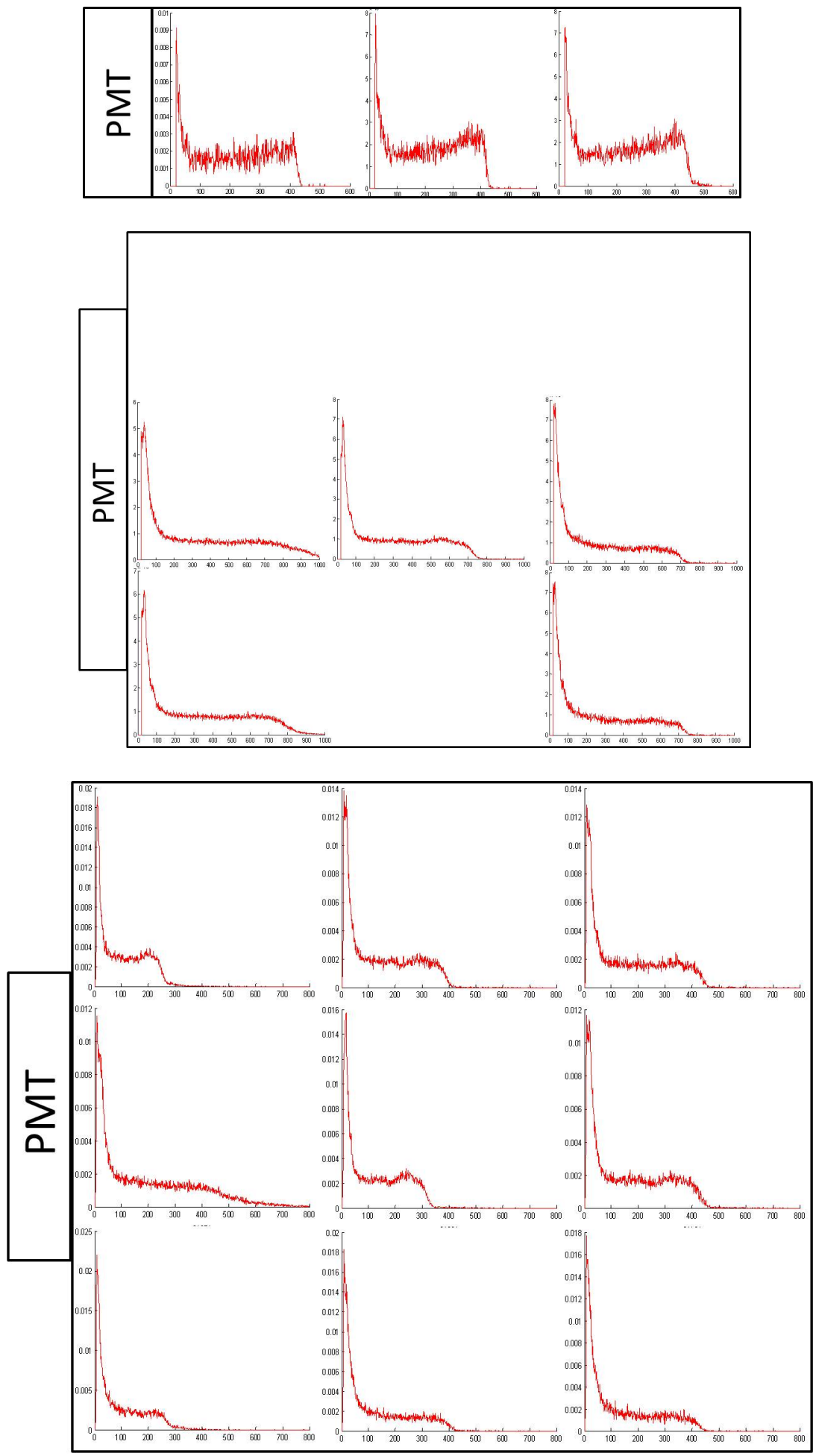

Figure A-4: Digitizer position specific detector responses for small (top), medium (middle), and large (bottom) detectors. 


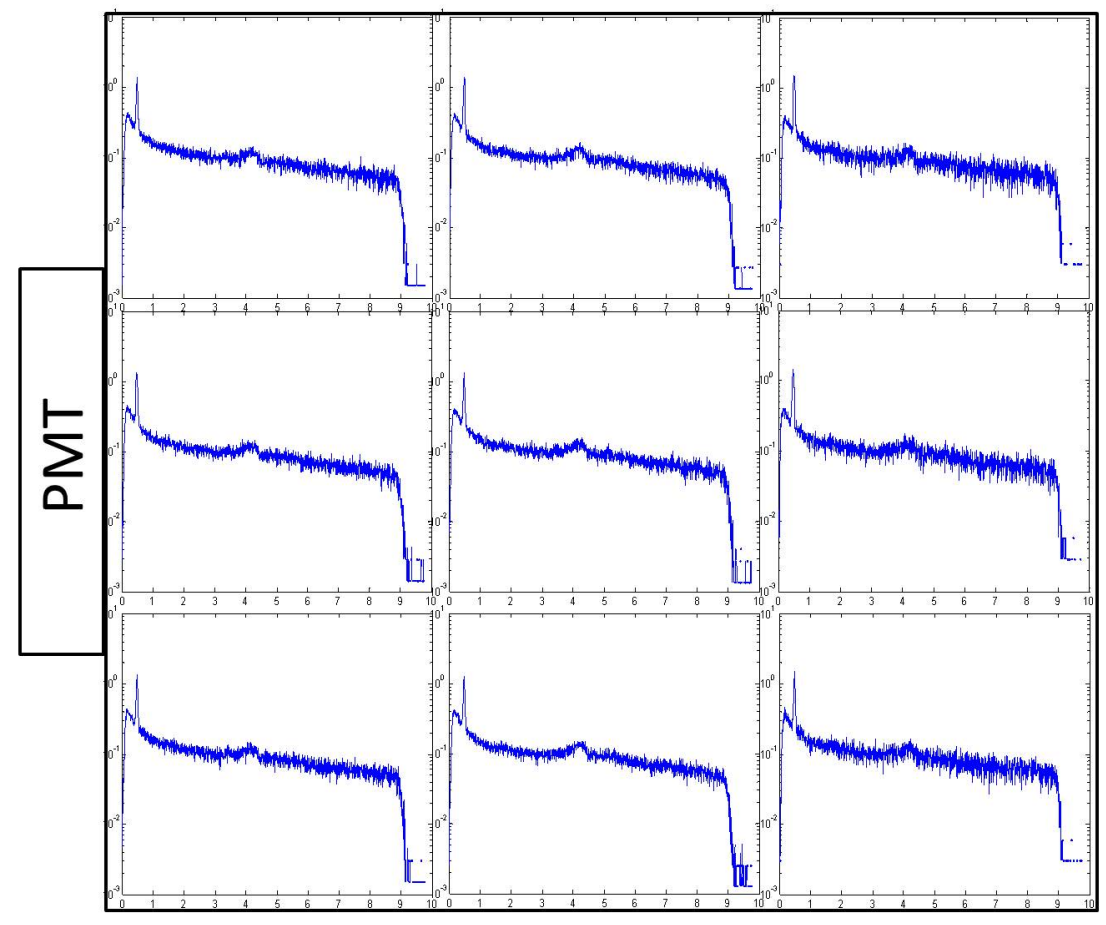

Figure A-5: Simulated position specific detector responses. 

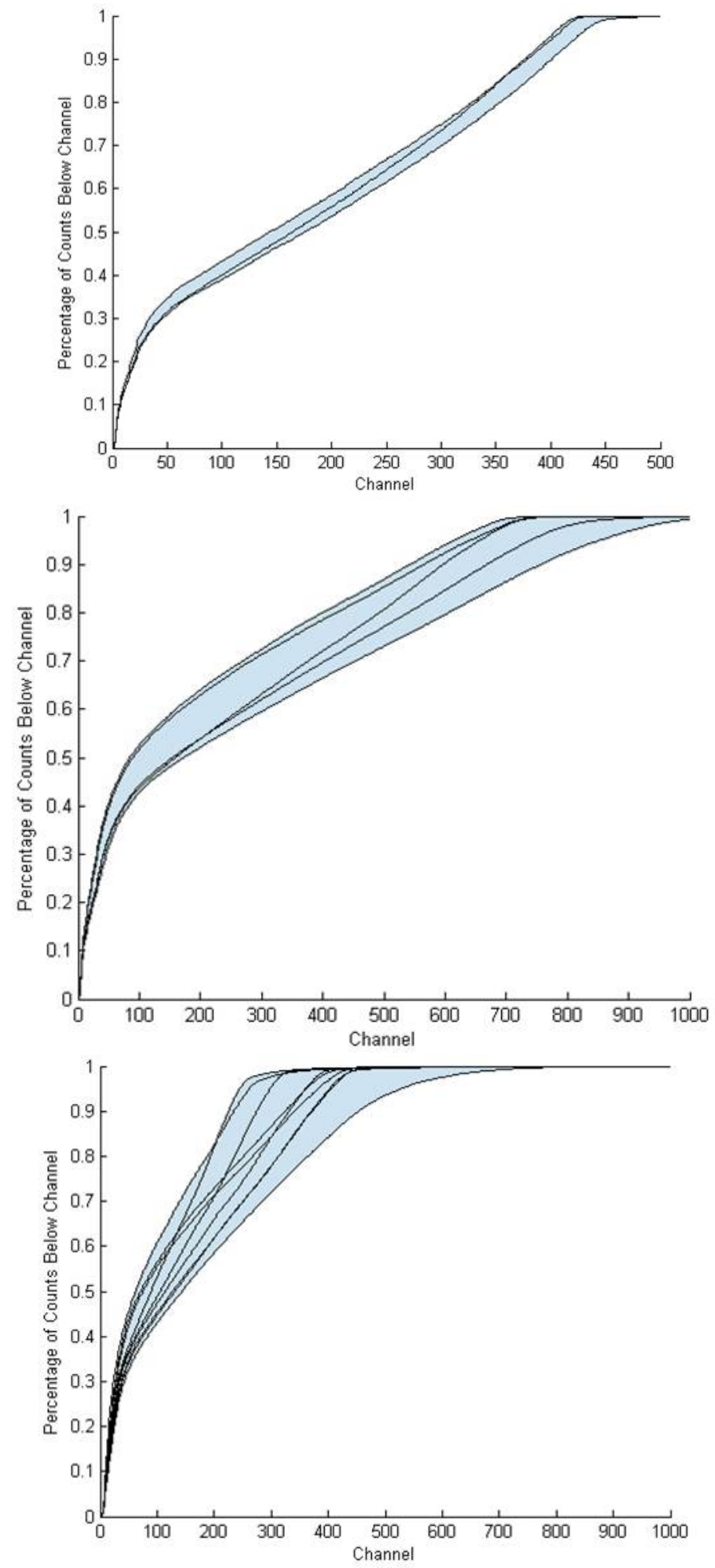

Figure A-6: Percentage of counts below each channel plots for small (top), medium (middle), and large (bottom) detectors shows the variation of pulse heights with position. 


\section{A.3 $\quad{ }^{137}$ Cs Detector Characterization}

\section{A.3.1 Experimental Setup}

In this section, a strong ${ }^{137} \mathrm{Cs}$ source was placed just inside of a narrow hole drilled through a tungsten block. This was a very effective collimator of the 667 $\mathrm{keV}$ photons emitted from the source. This source has only one particle emitted per decay, so it is not possible to use the associated particle method. However, since the source is so effectively collimated, it is still possible to single out small sections of the detector at a time. The collimated photon cone was characterized in the same method described in Chapter 4. The gamma source cone was determined to be about $3^{\circ}$ and circular, so that when a detector was placed $25 \mathrm{~cm}$ from the source opening in the tungsten block, the diameter of the source spot was about $1.3 \mathrm{~cm}$ (this cone included about $89 \%$ of photons).

Instead of doing a analog and digital probing of the detectors, this source was used to analyze the detectors more thoroughly with the digitzer. The detectors were scanned in a similar method as was done previously with the analog system; the source was rastor scanned in 1 inch steps across the detector face (vertical steps were automatic and horizontal steps involved moving the detector by hand). The source strength was high enough that the measurement time for each location was $2 \mathrm{~min}$ for the small detectors, $1.5 \mathrm{~min}$ for the medium detector, $1 \mathrm{~min}$ for the large detector, and $0.5 \mathrm{~min}$ for the very large detector.

\section{A.3.2 Results}

The results of the scanned measurements can be seen in Figures A-7 - A-10. The plots include the spectra taken at all locations within the detectors. They are 
plotted on top of each other in order to give a sense for the variation of detector response across all locations of interaction. The reduced magnitude is at times due to the source spot going off the edge of the detector, so magnitude is not necessarily a good indicator of detector response variation. The analysis instead focused on the effect of threshold settings on these detectors and the variation of Compton edge location. The red line on the plots represents a reasonable threshold setting. The color coded surface map of each detector face looks at the total percentage of counts above the threshold setting at each location. The statistics were good enough for these measurements that statistical uncertainty was not considered in this discussion.

The small detector results seen in Figure A-7 show some moderate levels of variation due to detector response. Since this plot is not correlated, there could be some scattered photons interacting in various parts of the detector. It is interesting that the closest pulse location to the PMT has a long tailing Compton edge, which will be seen in all detectors.

The medium detector results seen in Figure A-8 shows slightly more variation than seen in the small detector, especially when looking at the location of the Compton edge. The detector movement was likely slightly off-center to give more sensitivity to the left side of the detector than the right side; the source was likely further off the edge of the detector on the scan of the right side of the detector. Again, the tailing Compton edge spectra are seen for the interactions directly in front of the PMT, and the spectra with lower channel Compton edges are further from the PMT.

The large detector results seen Figure A-9 are the most detailed and really give a sense for the sensitive and insensitive regions of the detector face. The locations of the Compton edges vary significantly, which is reflected in the threshold study. The corners nearest the PMT edge are least visible by the PMT and therefore are the 


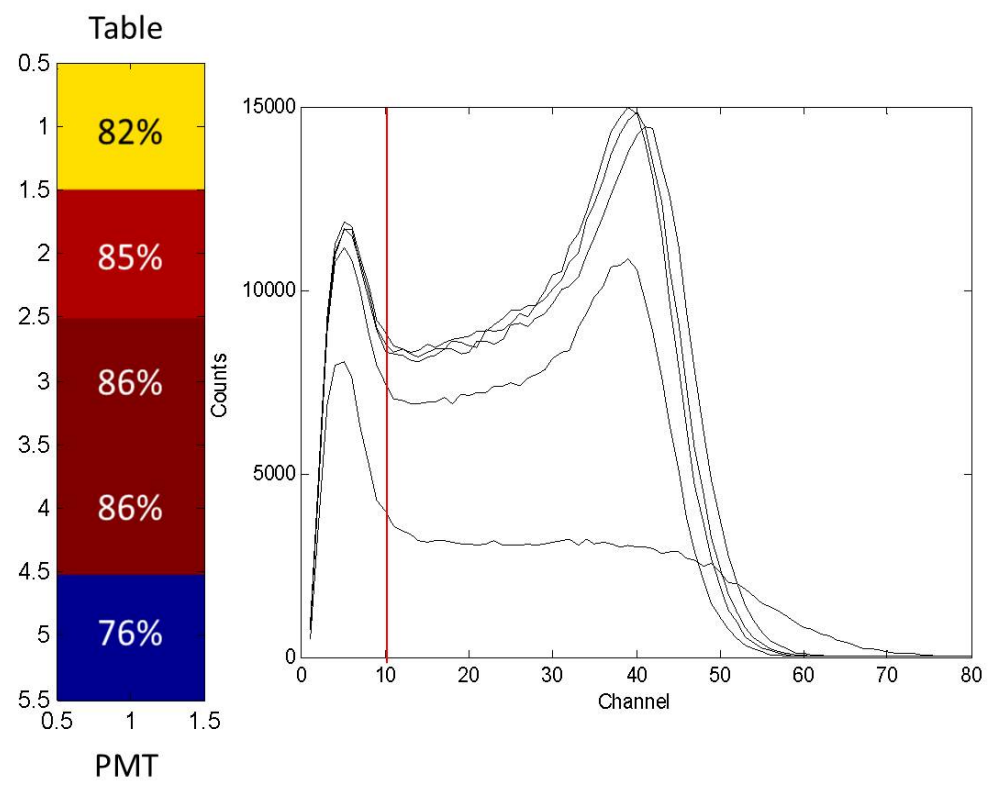

Figure A-7: The small detector response represented by all spectra taken for the various locations as well as a colormap showing the variation of threshold. The red line on the plot of the spectra shows the threshold setting use in the colormap.

least sensitive regions. The region directly in front of the PMT is the most sensitive region.

Figure A-11 selects a few spectra from the large detector in order to represent the spectra in the same style as in Figure A-4. It is reassuring that the behavior seen in both of these figures is very similar.

The very large detector was not scanned in quite as much detail as the previous detectors, only taking spectra every 2 inches instead of every inch. The results, shown in Figure A-10, are interesting because they appear to vary less with interaction location, besides the one spectrum from the position directly in front of the PMT which extends all the way to channel 300 . Overall, the behavior is very similar to other detectors. The lower amount of variation could be due to a more effective reflector surrounding the detector material.

The plots in Figure A- 6 done for the DT generator results were repeated for the 

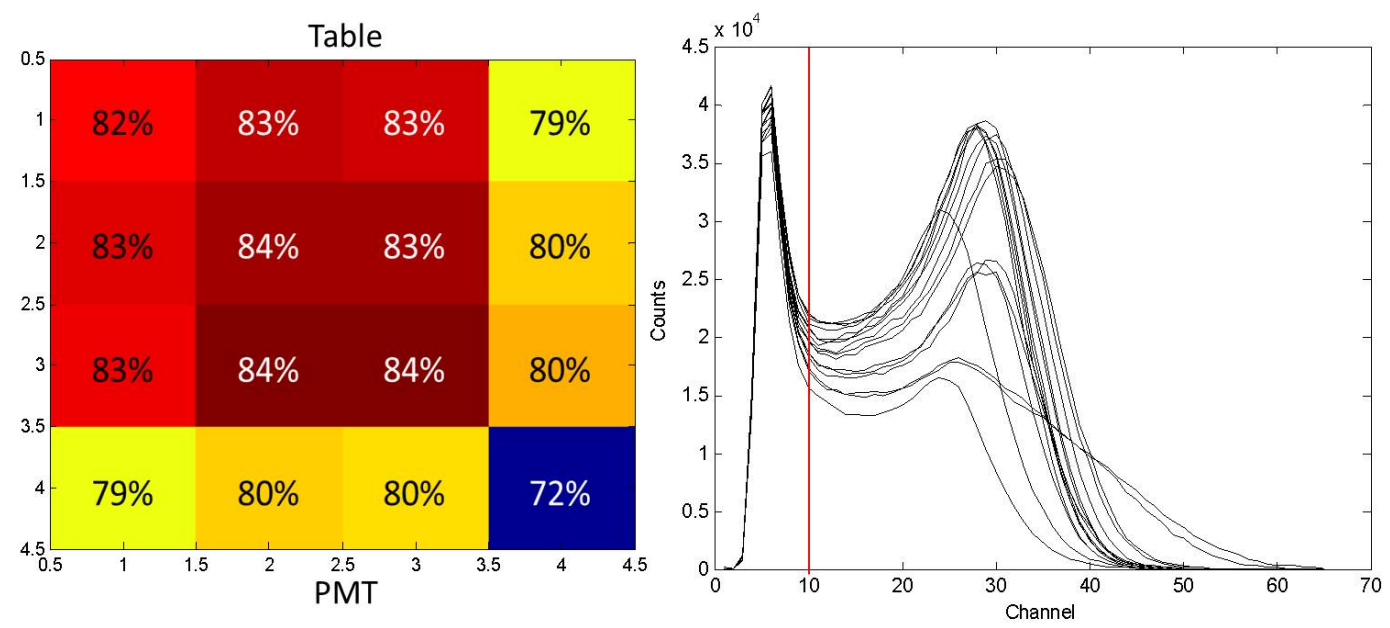

Figure A-8: The medium detector response represented by all spectra taken for the various locations as well as a colormap showing the variation of threshold. The red line on the plot of the spectra shows the threshold setting use in the colormap.

${ }^{137}$ Cs results. The series of plots for all detectors can be seen in Figure A-12, and are again effective at representing the amount of variation of the spectra for each detector. The amount of variation in detector response for the ${ }^{137} \mathrm{Cs}$ results appears to increase in a very similar way to the DT generator results. As could have been guessed by looking at Figure A-9 and A-10, the variation does not appear to increase for the very large detector when compared to the large detector. Again, this could be due to a the very large detector having a more effective reflector surrounding the detector material. 

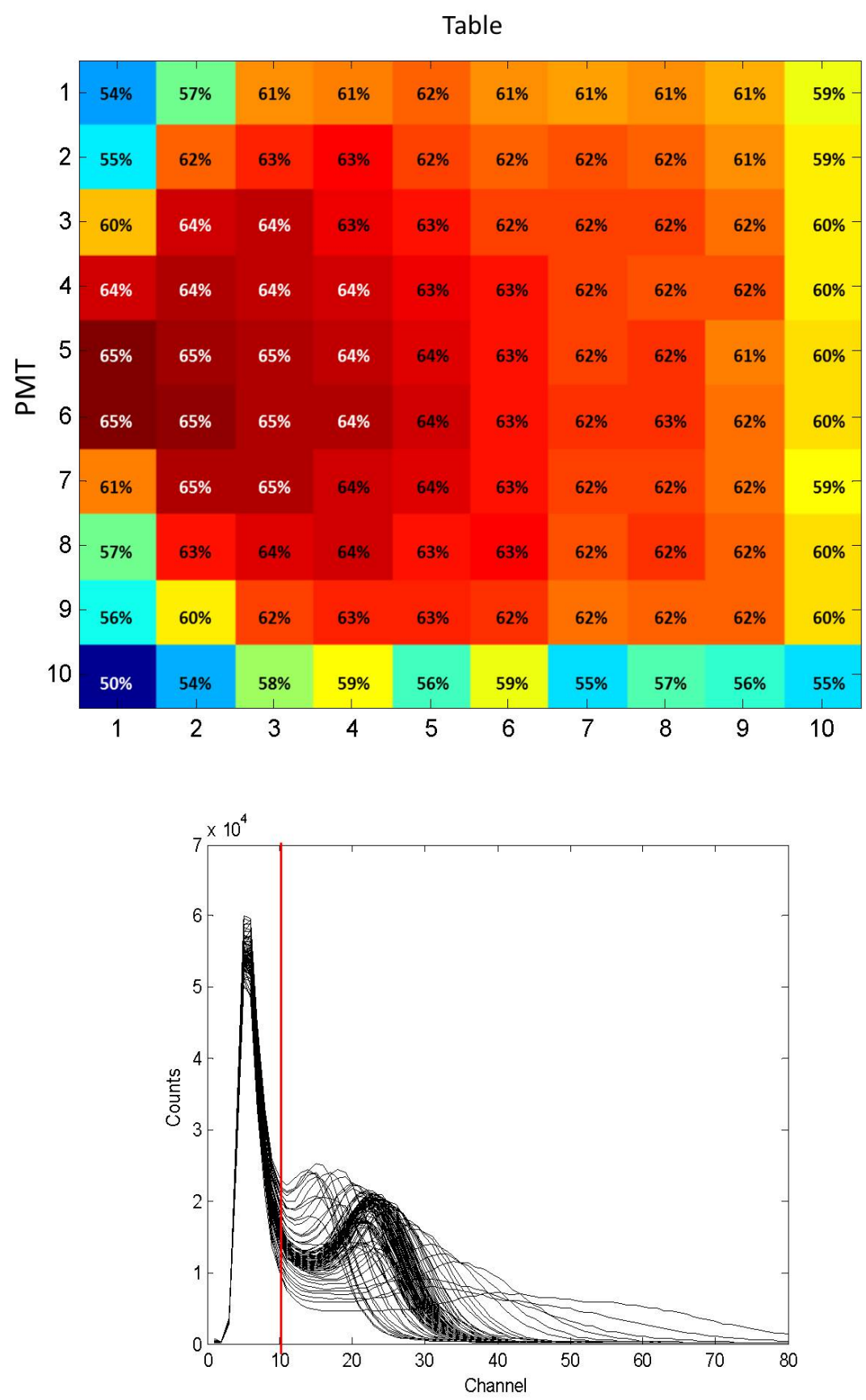

Figure A-9: The large detector response represented by all spectra taken for the various locations as well as a colormap showing the variation of threshold. The red line on the plot of the spectra shows the threshold setting use in the colormap. 


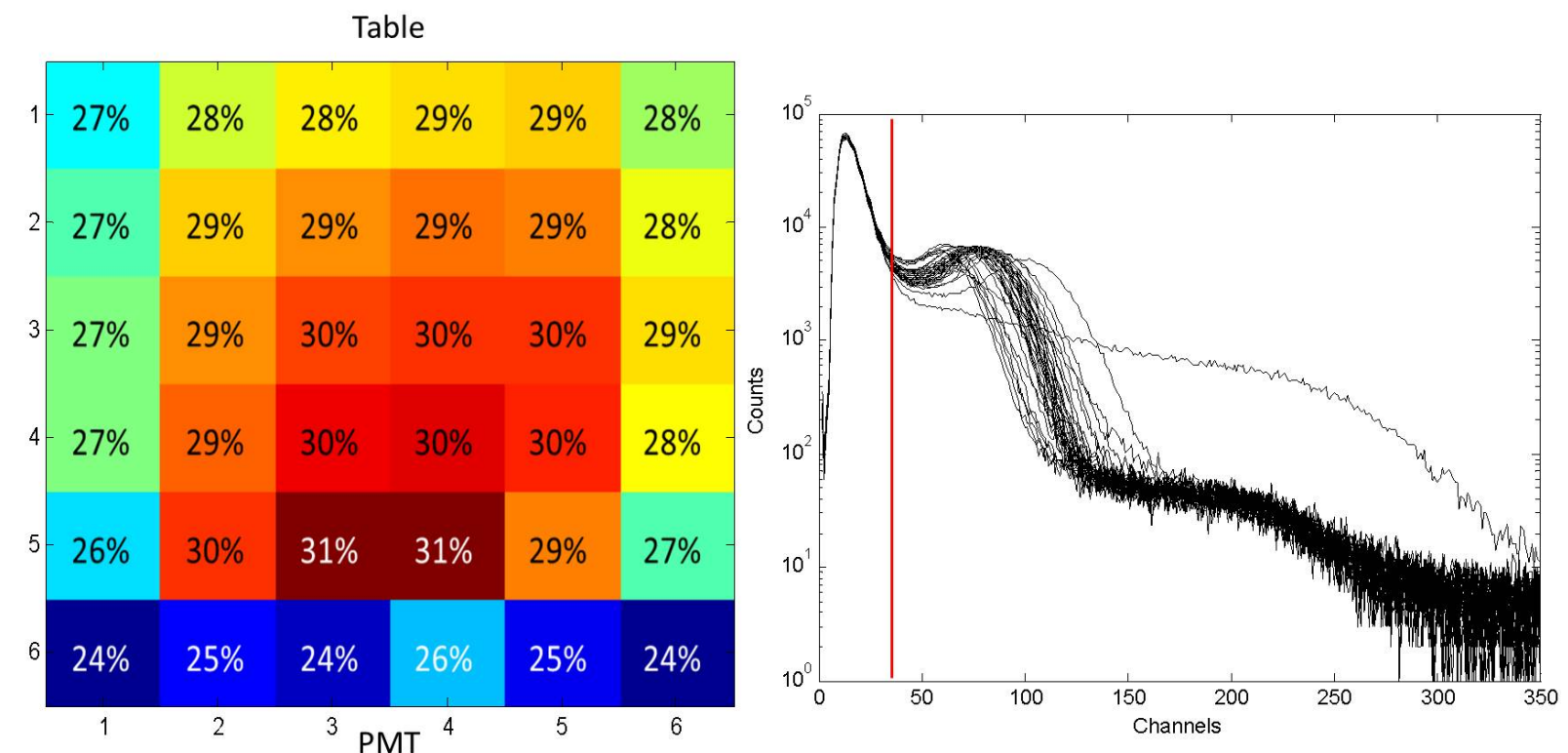

Figure A-10: The very large detector response represented by all spectra taken for the various locations as well as a colormap showing the variation of threshold. The red line on the plot of the spectra shows the threshold setting use in the colormap.

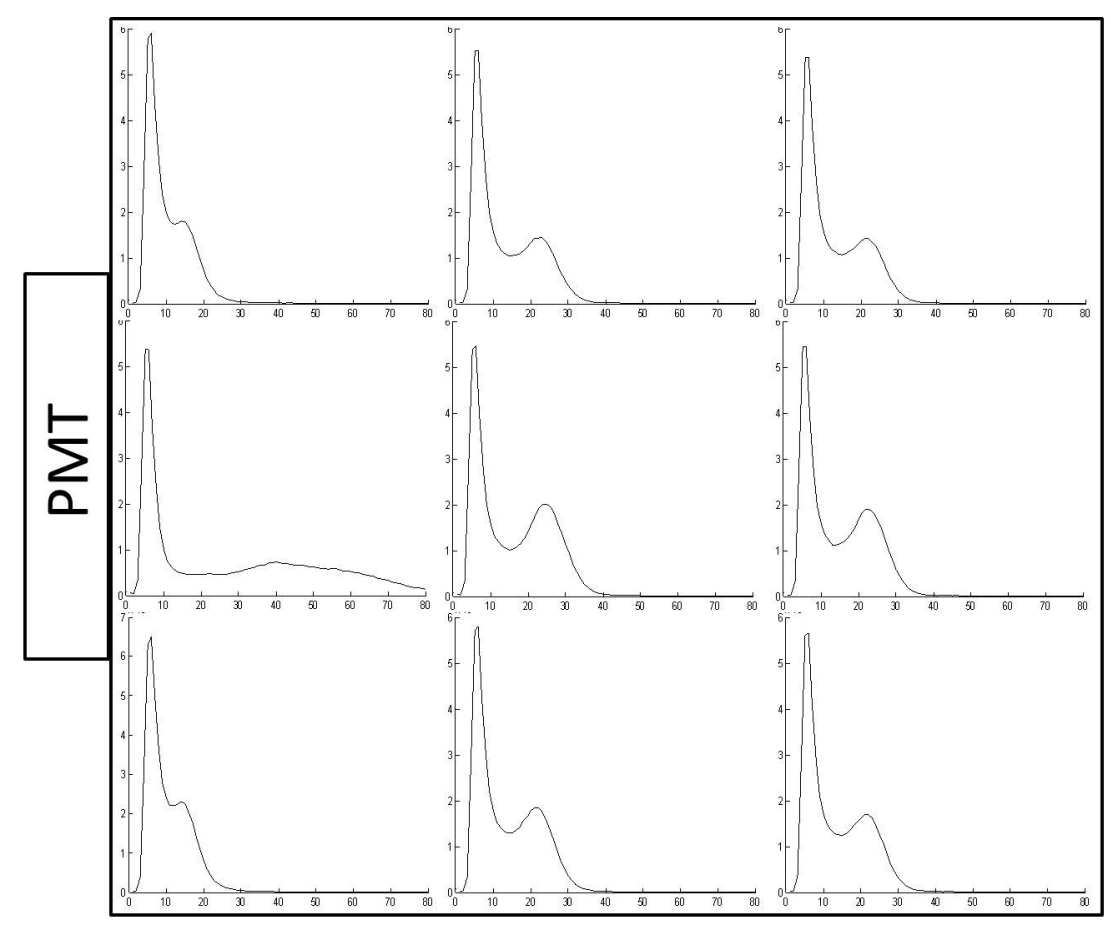

Figure A-11: Digitizer position specific detector responses for large detector, in the same style as Figure A-4. 

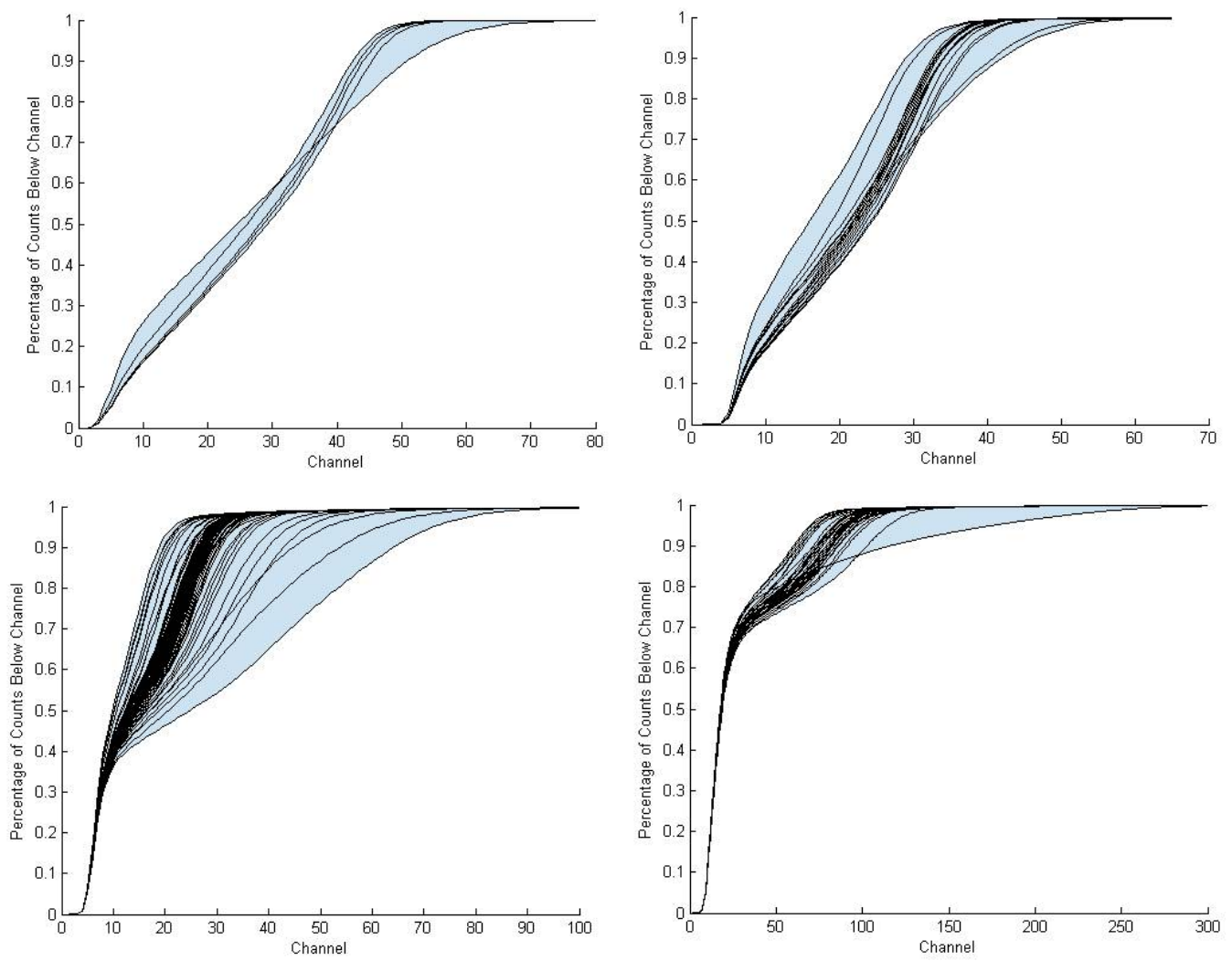

Figure A-12: Percentage of counts below each channel plots for small (top left), medium (top right), large (bottom left), and very large (bottom right) detectors shows the variation of pulse heights with position. 


\section{A.4 Modifications to Post Processor}

The post processor was modified to account for the variations seen in these detector scans. The scintillation light was not transported directly, but the effects of scintillation light seen in these experiments was approximated.

Two new input parameters were added into the post processor. The first defines the size and $(\mathrm{x}, \mathrm{y}, \mathrm{z})$ positions of each detector via translation-rotational matrix parameters, in the same form as the MCNP transport cards. The second input file was a matrix of relative light yields, based on location. The data from the scan of the large detector was used in this matrix, normalizing all positions to be factors of light intensity relative to the middle of the detector. For example, the middle of the detector is a factor of "1", the position in front of the PMT is greater than one, and the corners nearest the PMT are less than 1.

Based on these input files, the $(\mathrm{x}, \mathrm{y}, \mathrm{z})$ locations of each interaction are used to amplify or reduce the light yield slightly based on location. Because the middle of the detector is more sensitive than the average sensitivity of the detector as a whole, the overall detector efficiency was reduced to $87 \%$ of the original efficiency.

Figure A-13 shows the results of simulating the detector response of several positions on the face of a large detector. These simulation results are now much better aligned with the experimental scans of the large detector. 


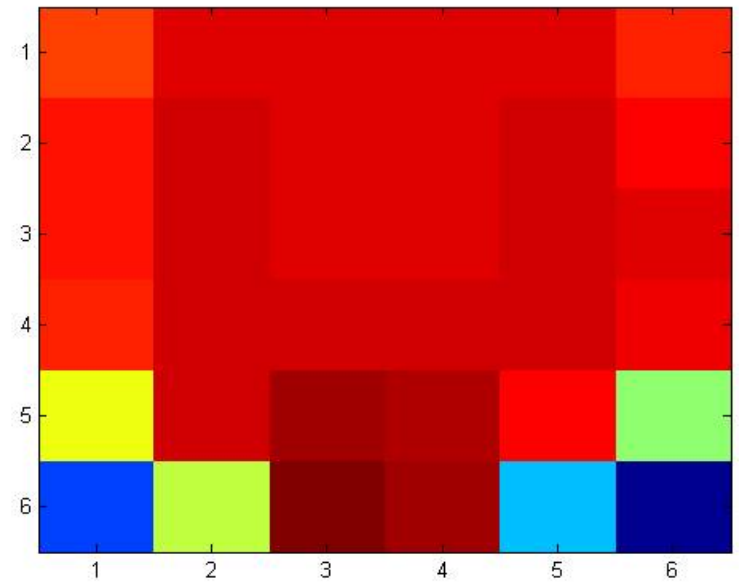

Figure A-13: Simulation of the detector response after modifying the post processor to account for position sensitivity. 


\section{B | Extended Experimental Setup}

This appendix details the steps taken to setup the API-DT generator and detectors used in Chapter 2. Additionally, some measurements were taken of the API-DT generator parameters in order to more accurately model the system in the MCNPPoliMi simulations, which are also detailed in this appendix.

\section{B.1 Calibrating the Fission Detectors}

In order to set the high voltage for these detectors, a ${ }^{137} \mathrm{Cs}$ point source is placed in the center of each detector. The CAEN Mod. N472 high voltage power supply is connected, and the detector annode signal is run into an oscilloscope. The high voltage is raised until the ${ }^{137} \mathrm{Cs}$ pulse is at a differential of about $-200 \mathrm{mV}$.

It is desirable to set the detector threshold in the Ortec Mod. 935 Quad CFD such that a majority of the fission neutron spectrum is above the threshold. Neutrons below the energy of $0.65 \mathrm{MeV}$ begin to be indistinguishable from background and floor return for this setup, so thresholds were set for a neutron energy of $0.65 \mathrm{MeV}$. The method for setting the detector threshold is to place all detectors onto the fission rack and place a fission chamber ${ }^{252} \mathrm{Cf}$ source in the center of the rack, such that it is equidistant from all detectors.

The fission chamber ${ }^{252} \mathrm{Cf}$ source design is used for time of flight measurements by using charged parallel plates to detect the fission products from a spontaneous fission interaction. The source pulses from the fission products are put into the 
NMIS acquisition system along with the fission detector pulses in order to synch up the timing and produce time-of-flight plots. Using these time-of-flight plots, along with the known radial distance and fission neutron energy spectrum of the ${ }^{252} \mathrm{Cf}$, an energy dependent detector efficiency curve can be generated to determine the neutron energy equivalent of the CFD threshold setting. The threshold settings on the CFD are adjusted until all detectors are set to the desired $0.65 \mathrm{MeV}$ threshold cutoff. Figure B-1 shows the efficiency curve for the average of all 8 fission detectors for their final threshold settings. Note that they high energy neutrons do not fit the curve well due to poor statistics (there are few neutrons greater than 3-4 MeV in the fission spectrum) and roughly grouped cross sections (detector material crosssections were used to correct for the thickness of the detector, but the cross sections were grouped into a small number of energy groups, which leads to inaccuracies). The highest energy neutrons in Figure B-1 likely also have some contributions from gamma-rays that were not separated well due time timing uncertainty.

Figure B-2 shows the californium time-of-flight plots for all 8 detectors with their final high voltage and threshold settings, with a summary of the settings included. Note that for these measurements, the rack has four detectors on a bottom row that is more likely to detect radiation scattered from the floor, which is why the background around $60 \mathrm{~ns}$ is higher in detectors $5-8$. Defining the photon peak as being from -2 to $13 \mathrm{~ns}$ and the neutron peak from 14 to $60 \mathrm{~ns}$, the photon and neutron peaks had a $6.4 \%$ and $5.2 \%$ standard deviation respectively between all 8 detectors. The threshold is marked by equating the time-of-flight of $0.65 \mathrm{MeV}$ neutrons. 


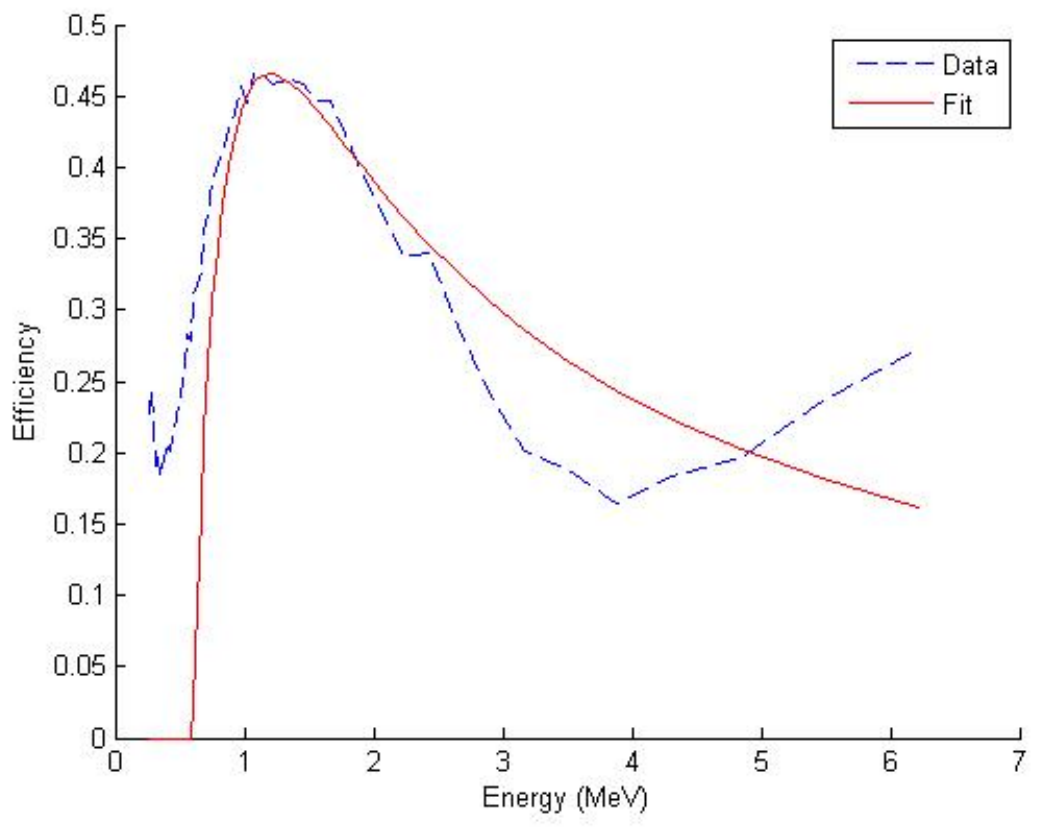

Figure B-1: Average efficiency curve for all eight detectors. 


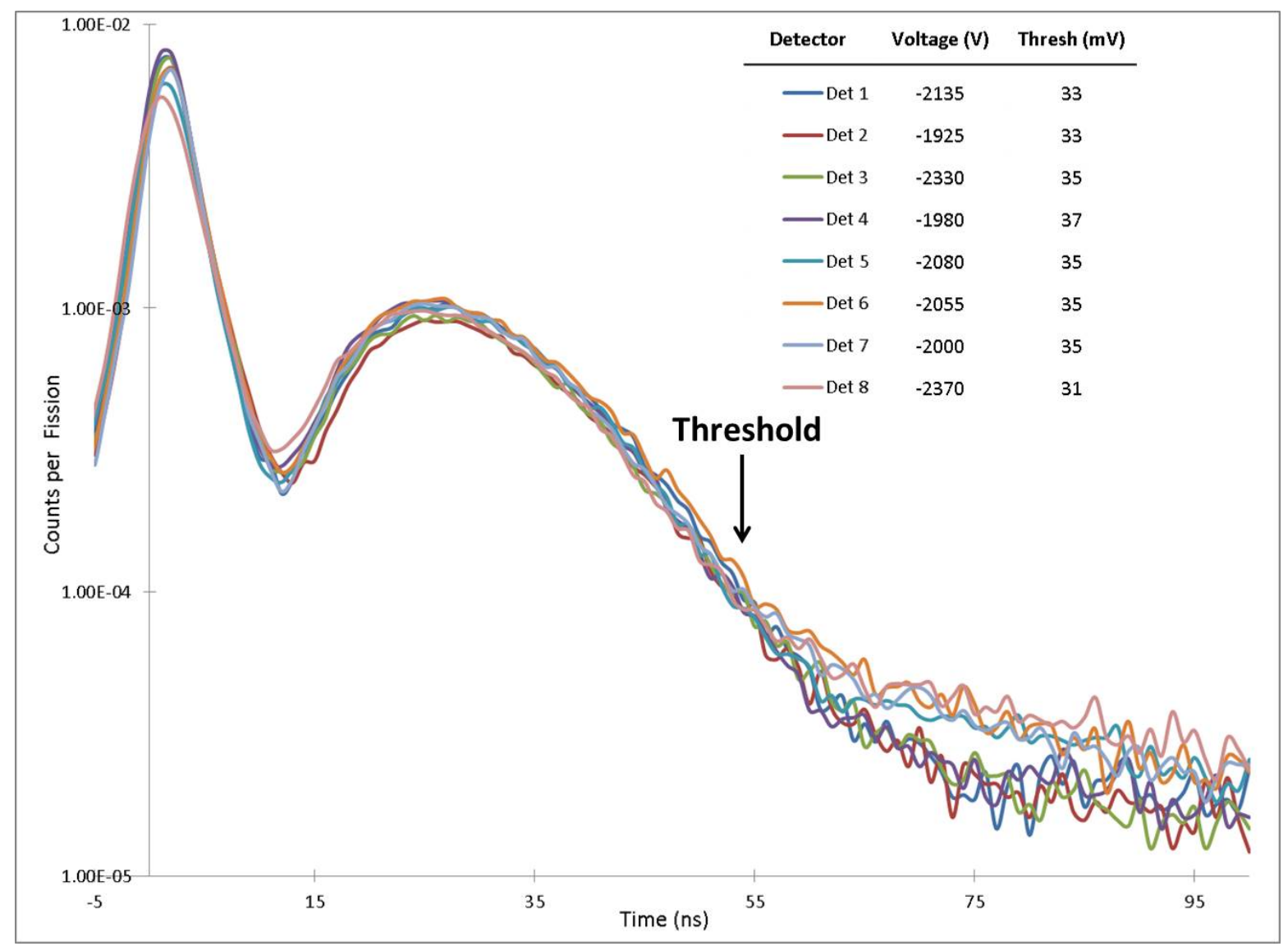

Figure B-2: time-of-flight plot using a ${ }^{252} \mathrm{Cf}$ source, used to calibrate detectors 


\section{B.2 Setting the Alpha Detector Threshold}

It is important to set each alpha pixel threshold in such a way that most of the non-alpha pulses are being rejected by the CFD and not many true alpha counts are being missed. In order to find this optimal point, a measurement is done with a single detector placed in front of the DT generator such that the whole neutron cone associated with the alpha pixel is hitting the detector. The pulses from the alpha pixel are sent through a splitter; half of the pulse (with a $5 \mathrm{k} \Omega$ resistor in series) goes to a Ortec 113 preamplifier, then a Ortec 673 spectroscopy amplifier, and finally a Tukan multichannel analyzer, which is connected to a computer. A spectrum is taken for several minutes of this half of the pulse. From this spectrum, shown in blue in Figure B-3, it is possible to see a large amount of background in the low energy region and a higher energy peak that consists of the true alpha pulses. In order to see the shape of just the alpha peak, the second half of the split alpha peak is put into a CFD and then a logic unit, along with the scintillation detector, which is also run through a CFD. Any coincidences between the alpha detector and the scintillation detector must be a true DT fusion events. These true events are run into channel A of the Tukan multichannel analyzer, which is used as a gate for the first signal of the alpha pulse. By taking a gated spectrum, only true alpha pulses are present in the spectrum, so that the shape of the alpha peak can be determined. Plotting the two spectra together and scaling the gated spectrum to fit into the ungated spectrum, it is possible to see what the rate of real alphas is. The results of this measurement are seen in Figure B-3, where the arrow shows the chosen location of the threshold. In order to set the CFD threshold to match this location, the total count rate at the chosen threshold is determined by integrating the total spectrum above this channel. The alpha pulses are then run through a 


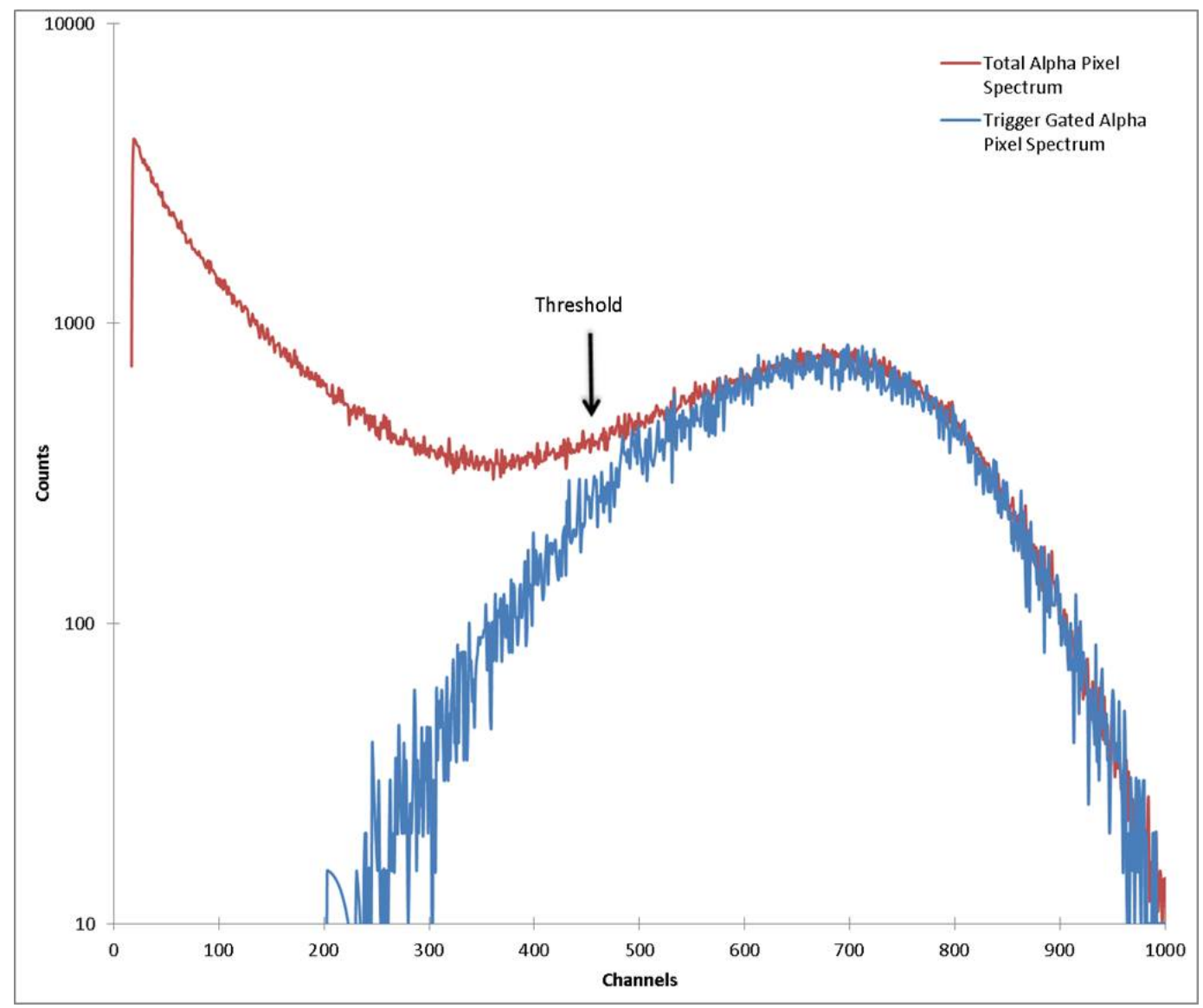

Figure B-3: Gated and total pulse height spectra from the alpha pixel used in all experiments. The location of the chosen threshold is labeled with an arrow.

CFD and then a counting unit, and the threshold on the CFD is varied until this rate is achieved. For the DT generator used with pixel 8 on the Hamamatsu, the HV setting was $-1050 \mathrm{~V}$ and the final threshold value was $-70.4 \mathrm{mV}$, which lead to an alpha rate with $90 \%$ true alpha counts and $10 \%$ background. 


\section{B.3 Accurately Modeling NMIS}

\section{B.3.1 Modeling the Neutron Cone}

In order to accurately model any active measurement taken by an API system, it is very important to understand the shape of the correlated neutron cone in order to input these parameters into the model. A separate measurement was done to characterize this shape. The neutron generator was placed on an immobile surface facing the imaging detector array, so that the imaging detector array could move independently of the DT generator and scan its correlated neutron field of view. The imaging detector array of NMIS (a semicircle of $321 " \times 1 " \times 4 "$ plastic detectors) took a series of measurements at a variety of heights, above and below the height of the DT generator. The imaging array also shifted angularly in order to increase the horizontal resolution at each vertical height. This scan created a two dimensional image of the correlated neutron cone, seen in Figure B-4.

In order to model this cone accurately, a series of simulations was done. The initial neutron cone shape was determined by fitting Gaussian curves to the horizontal and vertical components. A neutron cone with these dimensions was used in the source term of an MCNP-PoliMi simulation of this same scanning measurement. By simulating the measurement, the detector effects could be accounted for, such as the detector widths, cross talk, etc. The output of this simulated measurement was compared to the original measurement. The Gaussian fit parameters were adjusted and the simulation was redone until the simulation and data of the neutron cone matched. 


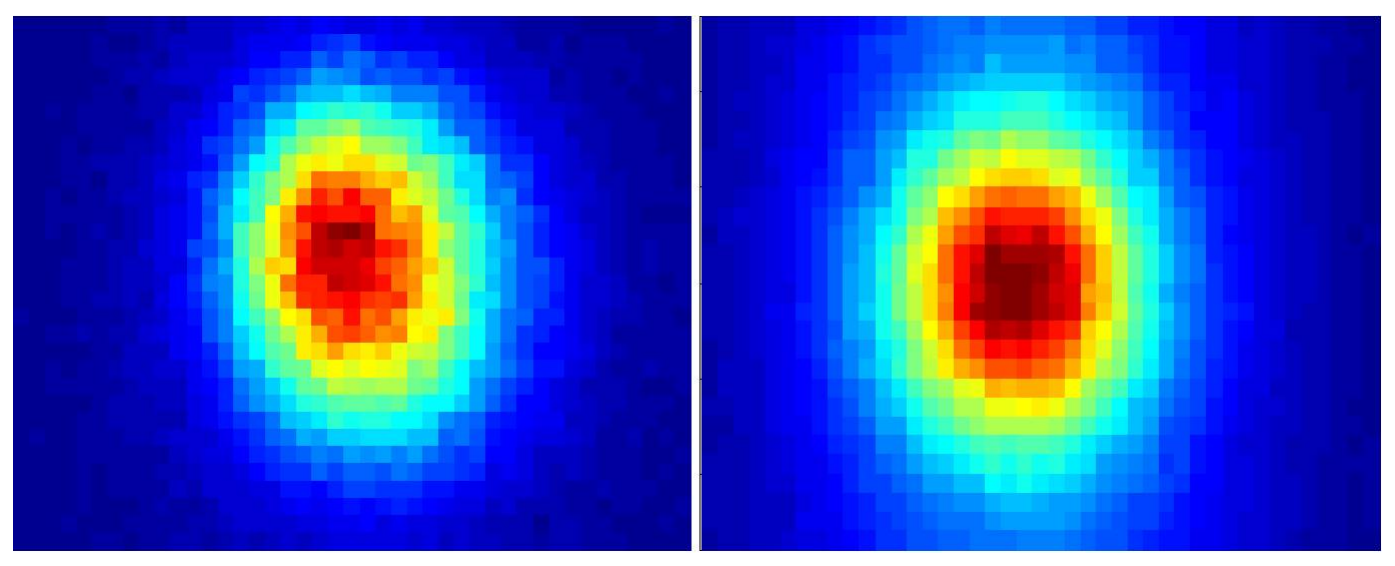

Figure B-4: Comparison of the correlated neutron spot as imaged by NMIS (left) and the Gaussian based fit (right). Note: these two figures are not exactly equivalent, as the left one has inherent detector effects not present in the right figure.

\section{B.3.2 Matching Detector Cross Talk}

One precaution taken to match the simulations to the experimental setup was to investigate the detector cross talk rates. A strong ${ }^{137} \mathrm{Cs}$ source was placed in the middle of the detector array. Since ${ }^{137} \mathrm{Cs}$ decays with only one gamma-ray at a time, any coincident counts recorded could be assumed to be cross-talk between detectors. There were plastic blocks between detectors to reduce the cross talk, but some cross talk is inevitable. The correlation rate between detectors was determined to be about $5.9 \%$ of the total counts.

A simulation of this same experiment was done in order to assure a similar simulated detector array. Slight adjustments to the thicknesses of the plastic in between detectors were made. The final version of the simulated detector array gave a rate of $6.8 \%$ correlations per total counts. This was deemed a good level of compromise between closely simulating the actual shielding levels and matching the cross talk rates. 


\section{C | Correction For Source Location}

The purpose of this appendix is to show the calculation for the average distance a source neutron travels in a sphere. The source neutrons are modeled as being uniformly distributed in a sphere. This means that the distance traveled by a source neutron is the radial distance to the edge of the sphere starting from any point in a sphere and traveling in any direction from that point.

The average distance traveled by neutrons that are created uniformly in a sphere and travel isotropically from their location of origin is described by

$$
\bar{d}=\frac{3}{2 R^{3}} \int_{0}^{R} \int_{0}^{\pi}\left(\sqrt{R^{2}-r^{2} \sin ^{2} \theta}-r \cos \theta\right) r^{2} \sin \theta d \theta d r
$$

The terms used in this equation are illustrated in Figure C-1, where the expression in this volume integral is a representation of $d$ in terms of $r$ and $\theta$. Simplifying this equation slightly by substituting in $r=R * r^{\prime}$, where $r^{\prime}$ goes from 0 to 1 , the equation becomes:

$$
\bar{d}=\frac{3 R}{2} \int_{0}^{1} \int_{0}^{\pi}\left(\sqrt{1-r^{2} \sin ^{2} \theta}-r \cos \theta\right) r^{2} \sin \theta d \theta d r
$$

This integral was solved numerically. It was determined from this integral that the average distance a source neutron would travel to escape a sphere is $\approx 0.75 \times R$. 


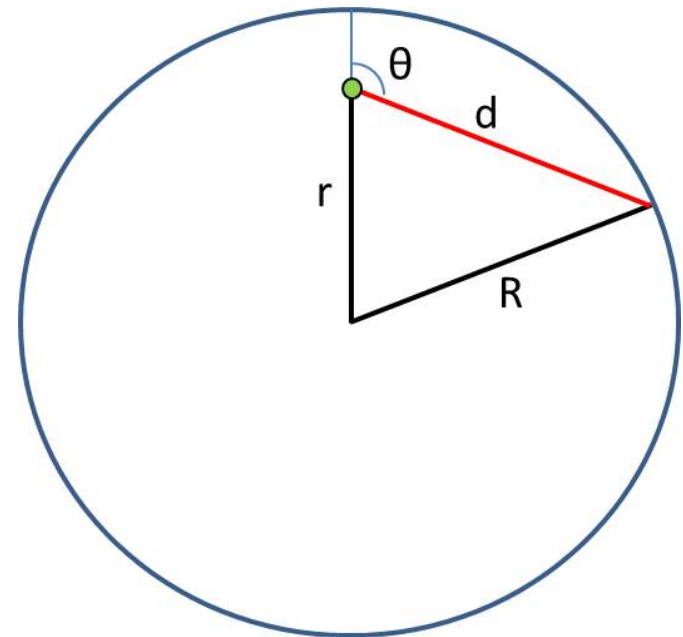

Figure C-1: This figure illustrates the setup used to build Equation C-1 


\section{D | High Purity Germanium Detec- tor Damage}

One of the primary considerations when using HPGe detectors in active interrogation techniques is minimizing detector damage. The phenomenon of HPGe detector damage has been well studied. The mechanism behind detector damage due to fast neutrons is the displacement of atoms in the lattice structure due to the energy deposited into the material. Depending on the amount of energy deposited, the damage to the lattice could be a simple Frenkel defect, meaning a vacancy and nearby interstitial due to a simple displacement in the lattice structure. However, when a larger amount of energy is deposited into the lattice, on the order of 20 $\mathrm{keV}$, a cascade of displacements occurs, resulting in a region of disorder (69). This region of disorder will typically have a negative charge, attracting holes (created by gamma-rays that are being detected) as they move through the material. The holes are trapped by the ionized region of disorder, leading to the charge being delayed and dissipating kinetic energy in the process. This slowing of the hole movement causes the spectroscopy peak to have a low energy tail, which severely reduces the resolution of the detector as this effect increases over time.

Since the damage leads to impaired motion of the holes, coaxial n-type Ge detectors are the most resistant to these effects (70). In this detector design, the $\mathrm{p}+$ contact is on the outer surface of the detector. Since the gamma radiation is more likely to interact on the outer surface of the detector than the inner surface, the 
holes have to move a shorter distance than the more mobile electrons, reducing the effects seen from the damages to the detector lattice.

Based on the understanding of the mechanism behind neutron damage to $\mathrm{Ge}$ detectors, it is not surprising that neutron energy plays a part in the amount of damage seen from a given neutron dose to a detector. As an example, Borrel et. al. (71) found that "for the ${ }^{60} \mathrm{Co} 1332 \mathrm{keV}$ peak, a degradation of the FWHM of $30 \%$ is achieved with a dose equal to more than $2.5 \times 10^{9} \mathrm{n} / \mathrm{cm}^{2}$ for neutrons of $5 \mathrm{MeV}$, $10^{9} \mathrm{n} / \mathrm{cm}^{2}$ for neutrons of $16 \mathrm{MeV}$ or $5 \times 10^{8} \mathrm{n} / \mathrm{cm}^{2}$ for neutrons of $6-70 \mathrm{MeV}$." A study done by Pehl et. al. (70) showed that for fission energy neutrons, the FWHM increased by about $40 \%$ after a dose to the detector on the order of $10^{10}$.

For the experimental setup being used for the application in Chapter 4, the DT generator source might be run at a power on the order of $5 \times 10^{7}$ neutrons/sec. In these initial measurements, the HPGe detector was about $65 \mathrm{~cm}$ away from the source, which reduces the flux reaching the $7 \mathrm{~cm}$ diameter and $9 \mathrm{~cm}$ long detector crystal to around $0.1 \%$ of the total flux based on solid angle. The shielding of a $30 \mathrm{~cm}$ cone of tungsten and an additional $10 \mathrm{~cm}$ of lead further reduces the source neutron flux to around $0.06 \%$ of the original flux reaching the crystal, based on an MCNP calculation. This leads to a negligible level of source neutrons reaching the detector crystal. The primary damage done to the crystal will be from the neutrons originating from the inspection object. Since these will be lower energy neutrons than the source neutrons, the damage level will be less severe. From these calculations, it is not expected that detector damage will be a significant problem for these purposes. 


\section{E | PMT Gain Shift}

When the neutron generator is turned on, the NaI detector goes from detecting mild background levels of radiation to suddenly having a much higher count rate. This is due to the higher radiation environment, from source neutrons as well as scattered or secondary radiation reaching the detector volume. It has been well studied that abrupt count rates in a detector volume cause significant gain shifts in the PMT. The mechanisms behind this behavior is discussed in citations (72; 73; 74). The gain shifts in the PMT cause the pulse height associated with gamma-ray energy to shift, leading to degraded resolution in the pulse height spectra. Luckily, the gain shifts in a predictable way that can be corrected for.

The rate at which this gain shift occurs follows a logarithmic function that is dependent on both the count rate change and the PMT properties. The count rate in the detector is dependent on the detector efficiency, as well as the flux and energy profile of the radiation reaching the detector, which means that the gain shift correction should be redone for changes in the detector setup since this would affect the count rate. Since the gain shift is a logarithmic function, the rate of change of the pulse height shift reduces after some time. For the setup used in this study, the neutron generator would be turned on for 15 - 20 minutes to allow the PMT gain to shift before any measurements were taken, so that gain was shifting at a low enough rate that it would likely be negligible for short measurements.

The measurements taken for this research were up to 60 minutes long, so effort was made to correct for the gain shift behavior. As soon as the DT neutron generator 


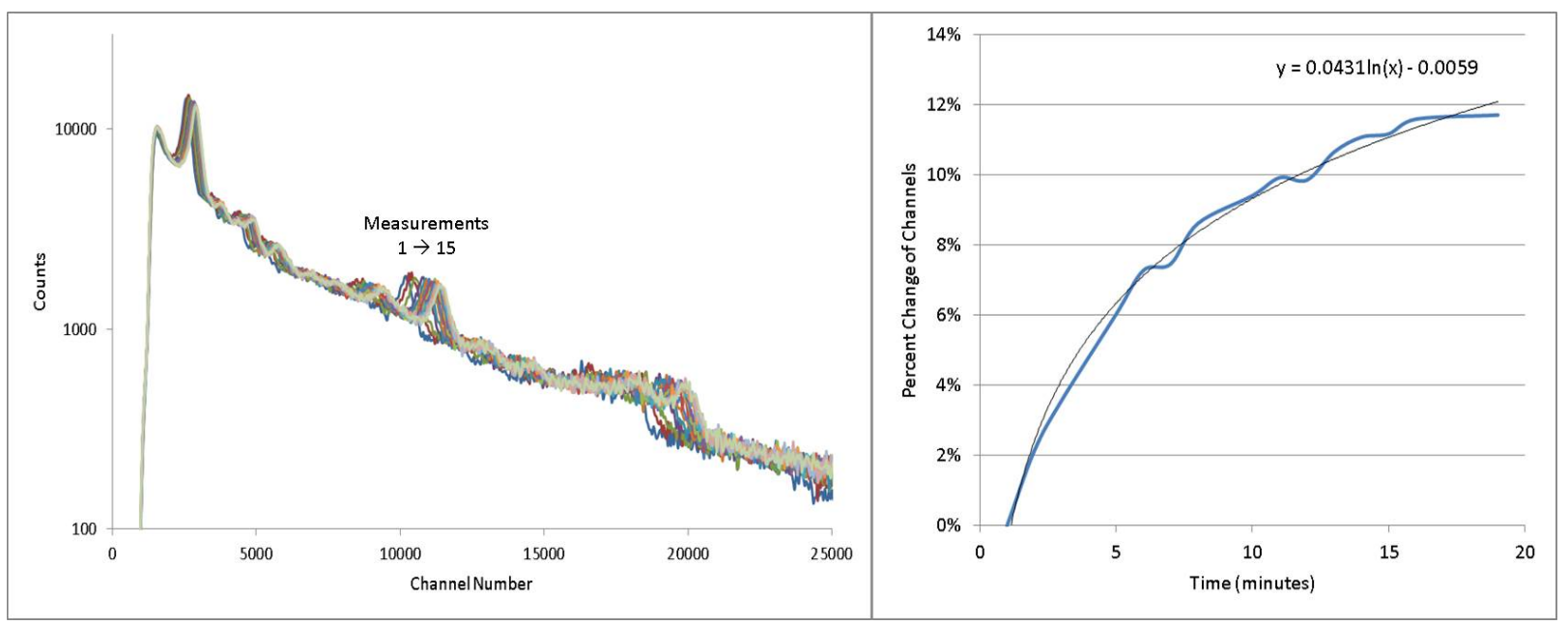

Figure E-1: The spectra (left plot) demonstrate the change of the pulse heights as the PMT gain shifts due to increased counts. The right plot shows pulse height shifts of NaI pulses over time.

was turned on, a series of 1 minute long measurements were taken back-to-back with a polyethylene target in the neutron generator alpha pixels' field of view. The results of these measurements are shown in Figure E-1, and show the rate of change of the energy relationship to the channel number over time. The peak channel of three prominent peaks were determined for each measurement, and the percent change of the channel number vs time was determined.

Figure E-1 shows the resulting distribution of the percent change over time of the channel numbers. This logarithmic fit was used to correct for changing energy calibration in the measurements made. However, this distribution is unique to the specific detector setup being used for these series of measurements, and could even change slightly due to the target type, because of the effect on the radiation flux reaching the detector. For this reason, the neutron generator was still turned on for several minutes before taking any measurements, due to the large shift in the energy calibration that occurs in the first 15 minutes or so.

The correction equation and results of correcting for the gain shift is shown in 


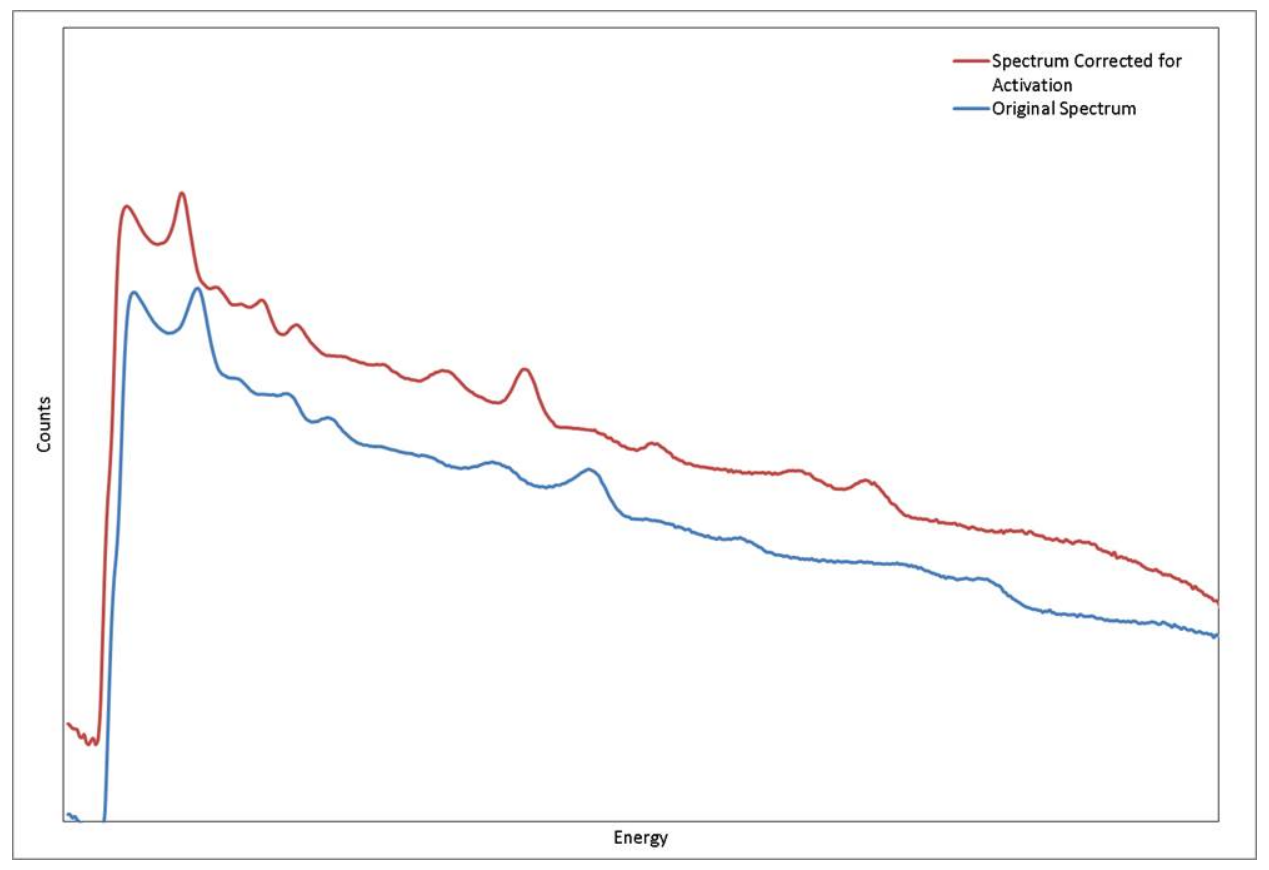

Figure E-2: A spectrum before and after being corrected for PMT gain shift. Spectra were offset from each other on the y-axis for visualization purposes.

Figure E-2. This spectrum shows how the peak resolution improves when this gain shifting effect is corrected for. 


\section{Bibliography}

[1] A. L. Swift, B. R. Grogan, J. A. Mullens, J. P. Hayward, and J. T. Mihalczo, "Attributes from NMIS Time Concidence, Fast-Neutron Imaging, Fission Mapping, and Gamma-ray Spectrometry Data," in Institute of Nuclear Materials Management Conference, (Oralando,FL), 2012.

[2] J. Mihalczo, P. R. Bingham, M. A. Blackston, J. M. Crye, B. R. Grogan, P. A. Hausladen, S. M. McConchie, and J. A. Mullens, "Fast-Neutron Imaging with API DT Neutron Generators," tech. rep., Oak Ridge National Laboratory, Oak Ridge, TN, 2012.

[3] D. Reilly, N. Ensslin, H. Smith, and S. Kreiner, Passive Nondestructive Assay of Nuclear Materials. Washington, DC: US Nuclear Regulatory Commission, 1991.

[4] K. J. Moody, P. M. Grant, and I. D. Hutcheon, Nuclear Forensics Analysis. Boca Raton: CRC Press, 2005.

[5] R. C. Runkle, D. L. Chichester, and S. J. Thompson, "Rattling Nucleons: New Developments in Active Interrogation of Special Nuclear Material," Nuclear Instruments and Methods in Physics Research Section A: Accelerators, Spectrometers, Detectors and Associated Equipment, vol. 663, pp. 75-95, Jan. 2012.

[6] Y. Bogolubov, S. Korotkov, L. Korytko, V. Morukov, V. Nazarov, Y. Polkanov, and T. Khasaev, "Method and System Based on Pulsed Neutron Generator 
for Fissile Material Detection in Luggage," Nuclear Instruments and Methods in Physics Research Section B: Beam Interactions with Materials and Atoms, vol. 213, pp. 439-444, Jan. 2004.

[7] J. Hall, S. Asztalos, P. Biltoft, J. Church, M.-a. Descalle, T. Luu, D. Manatt, G. Mauger, E. Norman, D. Petersen, J. Pruet, S. Prussin, and D. Slaughter, "The Nuclear Car Wash: Neutron Interrogation of Cargo Containers to Detect Hidden SNM," Nuclear Instruments and Methods in Physics Research Section B: Beam Interactions with Materials and Atoms, vol. 261, pp. 337-340, Aug. 2007.

[8] C. Hollas, C. Goulding, and W. Myers, "Determination of Neutron Multiplication of Subcritical HEU Systems Using Delayed Neutrons," Nuclear Instruments and Methods in Physics Research Section A: Accelerators, Spectrometers, Detectors and Associated Equipment, vol. 543, pp. 559-569, May 2005.

[9] S. Tobin, S. Demuth, J. Hendricks, H. Menlove, and M. Swinhoe, "Determination of Plutonium Content in Spent Fuel with NDA - Why an Integrated Approach is Needed," Institute of Nuclear Materials Management, 2008.

[10] G. A. Warren, R. S. Detwiler, and P. N. Peplowski, "Applications of Nuclear Resonance Fluorescence," in IEEE Nuclear Science Symposuim \& Medical Imaging Conference, (Knoxville, TN), pp. 534-541, 2010.

[11] T. Gozani, J. Stevenson, and M. J. King, "Neutron Threshold Activation Detectors (TAD) for the Detection of Fissions," Nuclear Instruments and Methods in Physics Research Section A: Accelerators, Spectrometers, Detectors and Associated Equipment, vol. 652, pp. 334-337, Oct. 2011. 
[12] J. T. Mihalczo, W. T. King, and E. D. Blakeman, "252Cf-Source-Driven Neutron Noise Analysis Method," in DOE Subcritical Reactivity Measurements Workshop, (Albuquerque, NM), 1985.

[13] J. L. Jones, W. Y. Yoon, K. J. Haskell, D. R. Norman, J. M. Hoggan, C. E. Moss, C. A. Goulding, C. L. Hollas, W. L. Myers, and E. Franco, "Photfission-based, Nuclear Material Detection: Technology Demonstration," tech. rep., Idaho National Engineering and Environmental Laboratory INEEL/EXT-02-01406, 2002.

[14] J. W. Schumer, R. J. Commisso, D. D. Hinshelwood, D. Mosher, D. P. Murphy, P. F. Ottinger, S. Member, D. G. Phipps, S. J. Stephanakis, B. V. Weber, F. C. Young, G. Cooperstein, B. W. Blackburn, J. L. Jones, J. Johnson, and N. O. Valles, "Development of an Intense Pulsed Characteristic $\gamma$-Ray Source for Active Interrogation of Special Nuclear Material," in IEEE Nuclear Science Symposium \& Medical Imaging Conference, (Honolulu, HI), pp. 1026-1032, 2007.

[15] T. Gozani, "Fission Signatures for Nuclear Material Detection," IEEE Transactions on Nuclear Science, vol. 56, pp. 736-741, June 2009.

[16] K. a. Jordan and T. Gozani, "Pulsed Neutron Differential Die Away Analysis for Detection of Nuclear Materials," Nuclear Instruments and Methods in Physics Research Section B: Beam Interactions with Materials and Atoms, vol. 261, pp. 365-368, Aug. 2007.

[17] T. Gozani, Active Nondestructive Assay of Nuclear Materials: Principles and Applications. National Technical Information Service, U.S. Department of Commerce, 1981. 
[18] B. W. Blackburn, J. L. Jones, C. E. Moss, J. T. Mihalczo, A. W. Hunt, P. Hausladen, S. a. Pozzi, F. J. Harmon, L. Waters, K. Inakiev, C. McCluskey, M. Flaska, S. M. Watson, and J. T. Johnson, "Actively-induced, Prompt Radiation Utilization in Nonproliferation Applications," 2006 IEEE Nuclear Science Symposium Conference Record, pp. 314-315, 2006.

[19] E. Rhodes, C. E. Dickerman, T. Brunner, A. Hess, and S. Tylinski, "Associated Particle Sealed Tube Neutron Generator Studies for Arms Control," tech. rep., Argonne National Loboratories, Argonne, IL, 1994.

[20] B. Perot, C. Carasco, C. Deyglun, C. Eleon, W. El Kanawati, A. Mariani, and J.1. Ma, "Materials Characterisation with the Associated Particle Technique," in 2012 IEEE Nuclear Science Symposium and Medical Imaging Conference Record (NSS/MIC), (Anaheim, CA), pp. 1702-1711, IEEE, Oct. 2012.

[21] L. F. Nakae, G. F. Chapline, A. M. Glenn, P. L. Kerr, S. Kim, S. A. Ouedraogo, M. K. Prasad, S. A. Sheets, N. J. Snyderman, J. M. Verbeke, and R. E. Wurtz, "Recent Developments in Fast Neutron Detection and Multiplicity Counting with Verification with Liquid Scintillator," in Conference on Nuclear Methods, (Crete, Greece), 2011.

[22] L. Lakosi, C. T. Nguyen, and E. Serf, "Neutron Interrogation of Shielded/Unshielded Uranium by a $4 \mathrm{MeV}$ LINAC," Applied Radiation and Isotopes : Including Data, Instrumentation and Methods for Use in Agriculture, Industry and Medicine, vol. 69, pp. 1251-4, Sept. 2011.

[23] H. H. Barschall, L. Rosen, and R. F. Taschek, "Measurement of Fast Neutron Flux," Reviews of Modern Physics, vol. 24, no. 1, pp. 1-14, 1952. 
[24] Y. Wu, J. P. Hurley, Q. Ji, J. W. Kwan, and K. N. Leung, "Sealed Operation of a RF Driven Ion Source for a Compact Neutron Generator to be Used for Associated Particle Imaging," The Review of Scientific Instruments, vol. 81, p. 02B908, Feb. 2010.

[25] S. Kane, T. Gozani, M. J. King, J. Kwong, C. Brown, C. Gary, M. I. Firestone, J. a. Nikkel, and D. N. McKinsey, "Simulations of Multi-Gamma Coincidences From Neutron-Induced Fission in Special Nuclear Materials," IEEE Transactions on Nuclear Science, vol. 60, pp. 533-538, Apr. 2013.

[26] D. S. Koltick, "The Integrated Detection of Hazardous Materials," AIP Conference Proceedings, vol. 680, pp. 835-839, 2003.

[27] D. V. Jordan, J. E. Baciak, S. C. Stave, D. Chichester, D. Dale, Y. Kim, and F. Harmon, "Associated Particle Tagging (APT) in Magnetic Spectrometers," Tech. Rep. October, Pacific Northwest National Laboratory PNNL-21913, Richland, WA, 2012.

[28] I. Dioszegi, C. Salwen, and P. Vanier, "Detection System for High Resolution Gamma Radiation Spectroscopy with Nuetron Time of Flight Filtering," 2012.

[29] A. V. Kuznetsov, D. N. Vakhtin, I. Y. Gorshkov, A. V. Evsenin, and O. I. Osetrov, "Using Associated Particle Technique for Detection of Shielded Nuclear Materials (NM) Detection of Heavily Shielded NM," in Countering Nuclear and Radiological Terrorism (S. Apikyan and D. Diamond, eds.), NATO Security through Science Series, ch. IV, pp. 255-269, Dordrecht: Kluwer Academic Publishers, 2006.

[30] V. M. Bystritsky, N. I. Zamyatin, E. V. Zubarev, V. L. Rapatsky, Y. N. Rogov, I. V. Romanov, a. B. Sadovsky, a. V. Salamatin, M. G. Sapozhnikov, M. V. 
Safonov, V. M. Slepnev, and a. V. Philipov, "Stationary Setup for Identifying Explosives Using the Tagged Neutron Method," Physics of Particles and Nuclei Letters, vol. 10, pp. 442-446, Sept. 2013.

[31] J. Mihalczo and J. Mullens, "Nuclear Material Identification System with Imaging and Gamma-Ray Spectrometry for Plutonium, Highly Enriched Uranium, High Explosives, and Other Materials," Tech. Rep. February, Oak Ridge National Laboratory, Oak Ridge, TN, 2012.

[32] J. A. Mullens, P. A. Hausladen, P. Bingham, D. E. Archer, and J. T. Mihalczo, "Use of Imaging for Nuclear Material Control and Accountability," ESARDA BULLETIN, no. 38, pp. 25-31, 2008.

[33] J. M. Crye, H. L. Hall, S. M. McConchie, J. Mihalczo, and K. E. Pena, "Enrichment Determination of Uranium in Shielded Configurations," in Institute of Nuclear Materials Management Conference, (Palm Desert, CA), Oak Ridge National Laboratory ORNL/TM-2012/77, 2011.

[34] J. M. Crye, Enrichment Determination of Uranium Metal in Shielded Configurations Without Calibration Standards. PhD thesis, Unniversity of Tennessee, 2013.

[35] J. A. Mullens, S. McConchie, P. A. Hausladen, J. T. Mihalczo, B. R. Grogan, and E. D. Sword, "Neutron Radiography and Fission Mapping Measurements of Nuclear Materials with Varying Composition and Shielding," in INMM 52nd Annual Meeting, (Desert Palm, CA), 2011.

[36] Table of Nuclides. http://atom.kaeri.re.kr/. 
[37] S. Pozzi, R. Oberer, and L. Chiang, "Higher-order statistics from NMIS to measure neutron and gamma ray cross talk in plastic scintillators," Nuclear Instruments and Methods in Physics Research Section A: Accelerators, Spectrometers, Detectors and Associated Equipment, vol. 481, no. 2002, pp. 739-748, 2002.

[38] S. Pozzi, S. Clarke, W. Walsh, E. Miller, J. Dolan, M. Flaska, B. Wieger, a. Enqvist, E. Padovani, J. Mattingly, D. Chichester, and P. Peerani, "MCNPX-PoliMi for nuclear nonproliferation applications," Nuclear Instruments and Methods in Physics Research Section A: Accelerators, Spectrometers, Detectors and Associated Equipment, vol. 694, pp. 119-125, Dec. 2012.

[39] B. R. Grogan, "The Development of a Parameterized Scatter Removal Algorithm for Nuclear Materials Identification System Imaging," tech. rep., Oak Ridge National Laboratory. ORNL/TM-2010/65, 2010.

[40] T. E. Valentine, "Evaluation of prompt fission gamma rays for use in simulating nuclear safeguard measurements," Annals of Nuclear Energy, vol. 28, no. 3, pp. 191-201, 2001.

[41] A. L. Swift, Materials and Configuration from NMIS Type Imaging and Gamma Spectroscopy. Masters of science, University of Tennessee, 2012.

[42] K. Bohnel, "The Effect of Multiplication on the Quantitative Determination of Spontaneously Fissioning Isotopes by Neutron Correlation Analysis," Nuclear Science and Engineering, vol. 90, pp. 75-82, 1985.

[43] R. Dierckx and W. Hage, "Neutron Signal Multiplet Analysis for the Mass Determination of Spontaneous Fission Isotopes," Nuclear Science and Engineering, vol. 85, pp. 325-338, 1983. 
[44] W. Hage and D. M. Cifarelli, "Correlation Analysis with Neutron Count Distributions in Randomly or Signal Triggered Time Intervals for Assay of Special Fissile Materials," Nuclear Science and Engineering, vol. 89, pp. 159-176, 1985.

[45] A. Enqvist, I. Pázsit, and S. Avdic, "Sample Characterization Using Both Neutron and Gamma Multiplicities," Nuclear Instruments and Methods in Physics Research Section A: Accelerators, Spectrometers, Detectors and Associated Equipment, vol. 615, pp. 62-69, Mar. 2010.

[46] L. Pál and I. Pázsit, "Neutron Fluctuations in a Multiplying Medium Randomly Varying in Time," Physica Scripta, vol. 74, pp. 62-70, July 2006.

[47] R. Oberer, Fission Multiplicity Detection with Temporal Gamma-Neutron Discrimination from Higher-Order Time Correlation Statistics. PhD thesis, Georgia Institute of Technology, 2002.

[48] D. Chernikova, K. r. Axell, S. Avdic, I. Pázsit, A. Nordlund, and S. Allard, "The neutronâgamma Feynman variance to mean approach: Gamma detection and total neutronâgamma detection (theory and practice)," Nuclear Instruments and Methods in Physics Research Section A: Accelerators, Spectrometers, Detectors and Associated Equipment, vol. 782, pp. 47-55, 2015.

[49] K. S. Kim, M. K. Prasad, L. Nakae, N. Snyderman, and J. M. Verbeke, "Time evolving fission chain theory and fast neutron and gamma-ray counting distributions," Nuclear Science and Engineering, vol. 181, pp. 1-53, 2015.

[50] P. A. Santi, T. E. Cutler, A. Favalli, K. E. Koehler, V. Henzl, D. Henzlova, R. F. Parker, and S. Croft, "Implementation and Initial Testing of Advanced Processing and Analysis Algorithms for Correlated Neutron Counting," tech. rep., Los Alamost National Laboratory Report LA-UR-15-29225, 2015. 
[51] S. McConchie, "Analytical Calculation of Fission Chain Probabilities in the Point-kinetics Framework," tech. rep., Oak Ridge National Laboratory, Oak Ridge, TN, 2011.

[52] M. Tweardy, A Point Kinetics Model for Neutron Coincidence Counting of Bare Uranium Metal in Tagged Neutron Measurement Systems. Masters, University of Tennessee, 2015.

[53] A. Enqvist, I. Pázsit, and S. Pozzi, "Analytical and Numerical Modelling of the Detection Statistics of Emission from a Fissile Sample with Absorption," in Joint International Topical Meeting on Mathematics \& Computation and Supercomputing in Nuclear Applications, (Monterey, CA), 2007.

[54] D. K. Hauck and V. Henzl, "Spatial Multiplication Model as an alternative to the Point Model in Neutron Multiplicity Counting," tech. rep., Los Alamos National Laboratory Report LA-UR-14-21991, 2014.

[55] S. Croft and A. Favalli, "Variations on and Extensions to Dytlewskiâs Dead Time Correction for Passive Multiplicity Counting.," tech. rep., Los Alamos National Laboratory Report LA-UR-14-29141, 2014.

[56] D. Hauck, S. Croft, L. G. Evans, A. Favalli, P. A. Santi, and J. Dowell, "Study of a theoretical model for the measured gate moments resulting from correlated detection evens and an extending dead time," Nuclear Instruments and Methods in Physics Research A, vol. 719, pp. 57-69, 2013.

[57] L. Bernstein and D. Brown, "Nuclear Data Needs and Capabilities for Applications White Paper," tech. rep., Lawrence Berkeley National Laboratory, Berkeley, 2015. 
[58] A. P. Barzilov, I. S. Novikov, and P. C. Womble, "Material Analysis Using Characteristic Gamma Rays Induced by Neutrons, Gamma Radiation," in Gamma Radiation, pp. 4-5, 2009.

[59] S. P. Simakov, A. Pavlik, H. Vonach, and S. Hlavac, "Status of Experimental and Evaluated Discrete Gamma-Ray Porduction at E=14.5 MeV," tech. rep., IAEA, Vienna, 1998.

[60] B. Perot, a. Mariani, E. Mercier, G. Sannie, G. Viesti, G. Nebbia, S. Pesente, M. Lunardon, P. Formisano, S. Moretto, D. Fabris, a. Zenoni, G. Bonomi, a. Donzella, a. Fontana, G. Boghen, V. Valkovic, D. Sudac, M. Moszynski, T. Batsch, M. Gierlik, D. Woski, W. Klamra, P. Isaksson, P. L. Tourneur, M. Lhuissier, a. Colonna, P. Peerani, V. Sequeira, and M. Salvato, "The EURITRACK Project : Status of a Tagged Neutron Inspection System for Cargo Containers," pp. 1-5, 2007.

[61] C. Carasco, C. Eleon, B. Pérot, K. Boudergui, V. Kondrasovs, G. Corre, S. Normand, G. Sannié, R. Woo, and J. M. Bourbotte, "Data acquisition and analysis of the UNCOSS underwater explosive neutron sensor," IEEE Transactions on Nuclear Science, vol. 59, no. 4 PART 2, pp. 1438-1442, 2012.

[62] D. N. Vakhtin, I. Y. Gorshkov, A. V. Evsenin, and O. I. Osetrov, "SENNA - Portable Sensor for Explosives Detection Based on Nansecond Neutron Analysis," in Detection and Disposal of Improvised Explosives (H. Schubert and A. Kuznetsov, eds.), NATO Security through Science Series, Dordrecht: Springer Netherlands, 2006.

[63] W. El Kanawati, B. Perot, C. Carasco, C. Eleon, V. Valkovic, D. Sudac, J. Obhodas, and G. Sannie, "Acquisition of Prompt Gamma-Ray Spectra Induced by 
$14 \mathrm{MeV}$ Neutrons and Comparison with Monte Carlo Simulations.," Applied Radiation and Isotopes : Including Data, Instrumentation and Methods for Use in Agriculture, Industry and Medicine, vol. 69, pp. 732-43, May 2011.

[64] M. E. Walker and J. T. Mihalczo, "Active Neutron Interrogation of NonRadiological Materials with the Nuclear Materials Identification System," Tech. Rep. February, Oak Ridge National Laboratory, Oak Ridge, TN, 2012.

[65] "Neutron Generators for Elemental Analysis of Substances and Materials," tech. rep., All Russia Research Institute of Automatics (VNIIA), Moscow.

[66] L. a. Currie, "Limits for Qualitative Detection and Quantitative Determination: Application to Radiochemistry," Analytical Chemistry, vol. 40, pp. 586-593, Mar. 1968.

[67] H. Klein and F. Brooks, "Scintillation detectors for fast neutrons," ..., International Workshop on Fast Neutron Detectors, ..., 2006.

[68] S. F. Naeem, S. D. Clarke, and S. a. Pozzi, "Spatial response characterization of liquid scintillator detectors using collimated gamma-ray and neutron beams," Nuclear Instruments and Methods in Physics Research, Section A: Accelerators, Spectrometers, Detectors and Associated Equipment, vol. 726, pp. 120126, 2013.

[69] L. S. Darken, R. C. Trammell, T. W. Raudorf, R. H. Pehl, and J. H. Elliott, "Mechanism for fast neutron damage of Ge(HP) detectors," Nuclear Instruments and Methods, vol. 171, pp. 49-59, Apr. 1980.

[70] R. H. Pehl, N. W. Madden, J. H. Elliott, T. W. Raudorf, R. C. Trammell, and L. S. Darken, "Radiation Damage Resistance of Reverse Electrode GE Coaxial 
Detectors," IEEE Transactions on Nuclear Science, vol. 26, no. 1, pp. 321-323, 1979.

[71] V. Borrel, B. Kandel, F. Albernhe, P. Frabel, B. Cordier, G. Tauzin, S. Crespin, R. Coszach, J. Denis, and P. Leleux, "Fast neutron-induced damage in INTEGRAL n-type HPGe detectors," Nuclear Instruments and Methods in Physics Research Section A: Accelerators, Spectrometers, Detectors and Associated Equipment, vol. 430, pp. 348-362, July 1999.

[72] R. Connor and M. Husain, "Some factors affecting the gain and resolution of photomultiplier tubes," Nuclear Instruments and Methods, vol. 6, pp. 337-342, 1960.

[73] A. K. Gupta and N. Nath, "Gain Stability in High Current Photomultipliers at High Variable Counting Rates," Nuclear Instruments and Methods, vol. 53, pp. 352-354, 1967.

[74] C. Zhong, H. Zhengde, and C. Qun, "Gain Stability of Photmultipliers at High Variable Counting Rates," Nuclear Instruments and Methods, vol. 281, pp. 384 387, 1989. 


\section{Vita}

Bonnie Elise Canion was born in Austin, Texas to Mikki McKune and David Canion. Growing up in Austin, she attended St. Andrew's Episcopal School throughout her elementary and high school education. After graduating high school in 2006, she attended Seattle University, where she majored in Physics with a secondary major in Mathematics. She received her Bachelor of Science degree in 2010. While working at an engineering firm in Austin, TX she applied and was accepted to the Nuclear and Radiation Engineering Program at the University of Texas in 2011, advised by Sheldon Landsberger. She completed her Masters in December 2012 and qualified for the $\mathrm{PhD}$ program shortly after. Upon completing her coursework, she completed the majority of her dissertation work at Oak Ridge National Laboratory in the Nuclear Material Detection and Characterization Group in Oak Ridge, TN, supervised by Seth McConchie. In June, 2015 she entered into the NNSA Graduate Fellowship Program where she was assigned to the Defense Nuclear Nonproliferation Research and Development Office (NA-22) in Washington, DC. She concurrently completed her dissertation, which she successfully defended on March 4th, 2016. Permanent email address: bcanion@gmail.com

This dissertation was typed by Bonnie Elise Canion. 